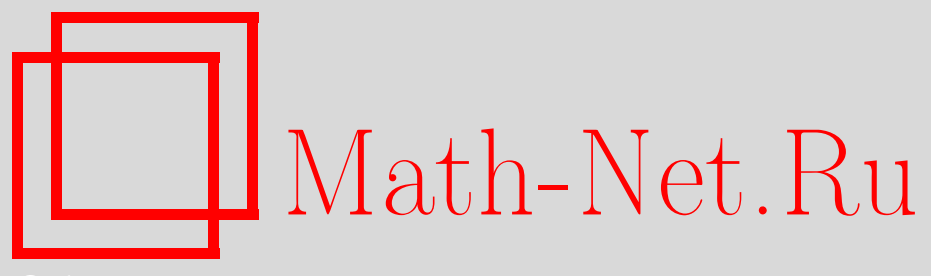

П. Б. Джаков, Б. С. Митягин, Зоны неустойчивости одномерных периодических операторов Шрёдингера и Дирака, УМH, 2006, том 61, выпуск 4, 77-182

DOI: https://doi.org/10.4213/rm2121

Использование Общероссийского математического портала Math-Net.Ru подразумевает, что вы прочитали и согласны с пользовательским соглашением http://www . mathnet.ru/rus/agreement

Параметры загрузки:

IP : 54.198 .55 .26

26 апреля 2023 г., 15:07:53

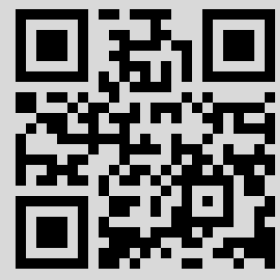




\title{
Зоны неустойчивости одномерных периодических операторов Шрёдингера и Дирака
}

\author{
П. Джаков, Б. С. Митягин
}

Спектры операторов Шрёдингера и Дирака с периодическим потенциалом на прямой $\mathbb{R}$ имеют зонную структуру, т.е. интервалы непрерывного спектра перемежаются спектральными лакунами, или зонами неустойчивости. Размеры этих зон убывают, и скорость этого убывания зависит от гладкости потенциала. Можно идти и в другую сторону и делать заключение о гладкости потенциала на основании того, как быстро убывают зоны неустойчивости. На уровне бесконечно-дифференцируемых или аналитических функций это явление в случае операторов Шрёдингера было понято в 60-е и 70-е годы. Однако только недавно соотношение между гладкостью потенциала и скоростью убывания зон неустойчивости в полном объеме было понято и проанализировано

- в широком диапазоне классов дифференцируемых функций,

- для операторов Дирака, а не только для операторов ШрёдингераХилла,

- как в самосопряженном, так и в несамосопряженном случае.

Обзору этих результатов, часто с полными доказательствами, основанными на развитой авторами методологии, и посвящена эта статья.

Библиография: 84 названия.

\section{СОДЕРЖАНИЕ}

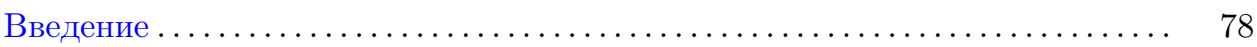

1. Локализация спектра и оценки резольвентных норм ............. 82

1.1. Основные обозначения и предположения; собственные значения и собственные функции в случае нулевого потенциала ....... 82

1.2. Формулы для резольвенты возмущенного оператора ......... 84

1.3. Матричное представление операторов умножения.......... 86

1.4. Оценки норм $\left\|R_{\lambda}^{0} V R_{\lambda}^{0}\right\| \ldots \ldots \ldots \ldots \ldots \ldots \ldots \ldots \ldots \ldots \ldots \ldots \ldots . \quad 89$

1.5. Оценки резольвентных норм и локализация спектра оператора Хилла-Шрёдингера; уклонение собственных подпространств

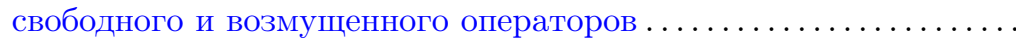

Первый автор благодарит за поддержку Научный фонд МОН Болгарии (договор ММ1401/04).

(C) П. Джаков, Б. С. Митягин, 2006 
1.6. Оценки резольвентных норм и локализация спектра оператора Дирака; уклонение собственных подпространств свободного и

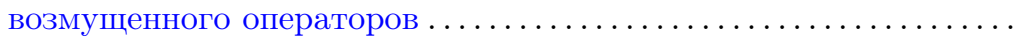



2. Основное уравнение; оценки для $\left|\gamma_{n}\right|$ сверху $\ldots \ldots \ldots \ldots \ldots \ldots \ldots \ldots \ldots . \ldots \ldots$

2.1. Редукционная схема.................................. 104

2.2. Основное уравнение: случай операторов Хилла-Шрёдингера . . . 107

2.3. Оценки $\alpha_{n}(v ; z)$ и $\beta_{n}^{ \pm}(v ; z)$ в случае операторов Хилла-Шрёдин-

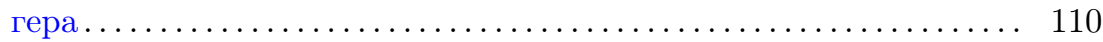

2.4. Основное уравнение: случай операторов Дирака ............ 114

2.5. Оценки параметров $\alpha_{n}(v ; z)$ и $\beta_{n}^{ \pm}(v ; z)$ в случае операторов Ди-

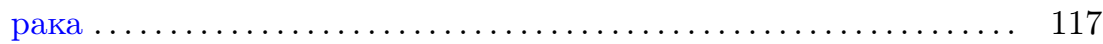

2.6. Оценки $\left|\gamma_{n}\right|$ сверху ................................ 127

2.7. Примечания . . . . . . . . . . . . 130

3. Обратные оценки в самосопряженном случае .................. 130

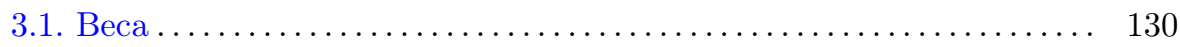

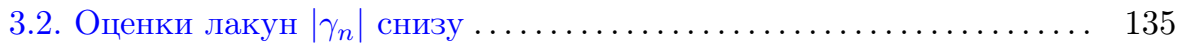

3.3. Гладкость потенциалов Хилла-Шрёдингера в самосопряженном случае ............................. 137

3.4. Гладкость потенциалов Дирака в самосопряженном случае .... 142

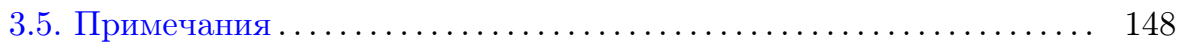

4. Несамосопряженный случай............................ 149

4.1. Предварительные леммы ........................... 149

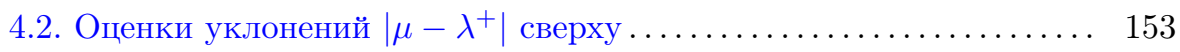

4.3. Оценки уклонения $|\gamma|+\left|\mu-\lambda^{+}\right|$снизу ................... 157

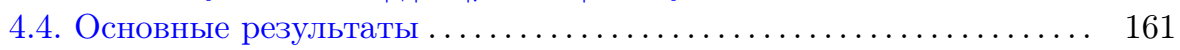

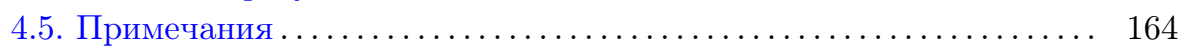

5. Заключительные замечания . . . . . . . . . . . . . . . . . . . 164

5.1. Плотность конечнозонных потенциалов ................ 164

5.2. Примеры расходящихся разложений по собственным функциям 166

5.3. Двучленные потенциалы . . . . . . . . . . . . . . . . . . . 171

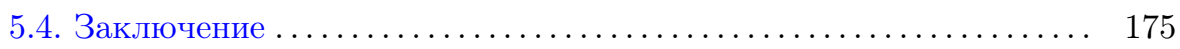

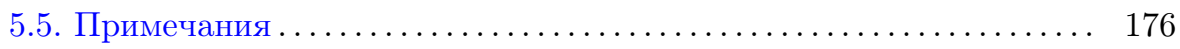

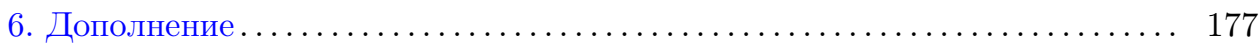

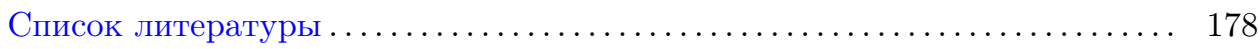

\section{Введение}

1. В анализе одномерных операторов Шрёдингера и Дирака существенную роль играют зоны неустойчивости, или спектральные лакуны, - интервалы между участками непрерывного спектра. Точнее, пусть

$$
L y=-y^{\prime \prime}+v(x) y, \quad x \in \mathbb{R},
$$

- оператор Шрёдингера с вещественнозначным периодическим потенциалом $v(x), v(x+\pi)=v(x), v \in L^{2}([0, \pi])$. Тогда спектр $\operatorname{Sp}(L)$ оператора $L$ состоит из 
интервалов $\left[\lambda_{n}^{+}, \lambda_{n+1}^{-}\right], n=0,1,2, \ldots$, причем

$$
-\infty<\lambda_{0}^{+}<\lambda_{1}^{-} \leqslant \lambda_{1}^{+}<\lambda_{2}^{-} \leqslant \lambda_{2}^{+}<\cdots .
$$

Если потенциал сдвигается, т.е. $v$ заменяется на $v_{\tau}(x)=v(x-\tau), \tau \in \mathbb{R}$, эта последовательность не меняется, так что семейство $v_{\tau}$ потенциалов изоспектралъно. Развитие методов обратной задачи теории рассеяния [1] в интегрировании нелинейных дифференциальных уравнений [2]-[8] дало новый толчок и специальный интерес в анализе периодического и антипериодического спектров операторов Шрёдингера и Дирака.

2. Особую роль в анализе уравнений Кортевега-де Фриза играют конечнозонные потенциалы и их римановы поверхности

$$
\mu^{2}=\left(\lambda-\lambda_{0}^{+}\right) \prod_{1}^{g}\left(\lambda-\lambda_{k}^{-}\right)\left(\lambda-\lambda_{k}^{+}\right) .
$$

Именно при попытке понять общую структуру конечнозонных потенциалов Г. Хохштадт [9]-[11] заметил прямую связь между гладкостью потенциала $v$ и скоростью убывания спектральных лакун $\gamma_{n}=\lambda_{n}^{+}-\lambda_{n}^{-}$. Если

(A) $v \in C^{\infty}$, т.е. $v$ бесконечно дифференцируема, Tо

(В) $\gamma_{n}$ убывает быстрее любой степени $1 / n$.

Если же непрерывная функция $v$ оказывается конечнозонным потенциалом, т.е. $\gamma_{n}=0$ для достаточно больших $n$, то $v \in C^{\infty}$. В середине $70-$ х годов [12], [13] последнее замечание было уточнено, т.е. было показано, что степенное убывание лакун влечет бесконечную гладкость потенциала, или $(\mathrm{B}) \Rightarrow(\mathrm{A})$ для вещественной $L^{2}$-функции $v$. Формула Гельфанда-Левитана [1] для следов и уравнения Дубровина [14], [3] были использованы Ю. Трубовицем [15] для объяснения того, что вещественнозначный $L^{2}$-потенциал $v(x)=\sum_{k \in \mathbb{Z}} V(2 k) \exp (2 i k x)$ аналитичен, т.е.

$$
\exists A>0:|V(2 k)| \leqslant M e^{-A|k|},
$$

тогда и только тогда, когда лакуны убывают экспоненциально быстро, т.е.

$$
\exists a>0: \gamma_{n} \leqslant C e^{-a|k|} .
$$

3. Хотя одномерные операторы Дирака (или системы Захарова-Шабата) также пришли в теорию вполне интегруемых систем в начале 70-х годов, соотношение между гладкостью потенциала и скоростью убывания лакун было рассмотрено только четвертью века позже [16]-[18]. Даже аналог критерия Хохштадта в случае оператора Дирака был дан только в 2003-05 гг. (см. [18], где результаты объявлены, и [19], где рассмотрен случай самосопряженного оператора Дирака). Одна из основных целей настоящей статьи - дать полное изложение этих результатов в случае несамосопряженного оператора Дирака.

4. Теория Флоке и Ляпунова объясняет [20]-[23], что граничные точки лакун находятся как собственные значения оператора Хилла

$$
L y=-y^{\prime \prime}+v(x) y, \quad x \in[0, \pi],
$$


с периодическими $\mathrm{Per}^{+}$или антипериодическими $\mathrm{Per}^{-}$граничными условиями на конечном отрезке $[0, \pi]$. Точнее, $\lambda_{n}^{+}, \lambda_{n}^{-}$при $n$ четном соответствуют $\mathrm{Per}^{+}, \mathrm{a}$ при $n$ нечетном $-\mathrm{Per}^{-}$. Потенциал Матье, т.е. $v(x)=2 \cos 2 x$, был важным примером и источником многих вопросов. Э. Л. Инс [24] показал, что в этом случае все периодические и антипериодические собственные значения однократны, т.е. $\gamma_{n}=\lambda_{n}^{+}-\lambda_{n}^{-} \neq 0, n \in \mathbb{Z}$. Точная асимптотика этой последовательности была найдена много позже (см. статьи Е. М. Харрела [25] и Й. Аврона и Б. Саймона [26]). Двучленный потенциал

$$
v(x)=a \cos 2 x+b \cos 4 x
$$

впервые появился в работе А. М. Ляпунова [27]. Авторам удалось дать точную асимптотику лакун в случае этого потенциала [28], [29]. Мы обсуждаем эти и примыкающие результаты в п. 5.3.

5. Если мы переходим к анализу операторов Хилла с комплекснозначными потенциалами, важно упомянуть наблюдение М. Г. Гасымова [30]. Если

$$
v(x)=\sum_{k=0}^{\infty} v_{k} e^{i k x}, \quad v \in L^{2}([0, \pi]),
$$

то все $\lambda_{n}$ двукратны, так что $\gamma_{n}=\lambda_{n}^{+}-\lambda_{n}^{-}=0$ (см. обсуждение в п. 5.3).

Это показывает, что само по себе убывание $\gamma_{n}$ не может дать никакого ограничения на гладкость $v$. Но теперь третье собственное значение $\mu_{n}$, скажем, при граничных условиях Дирихле $y(0)=y(\pi)=0$, становится контролирующим параметром. Наряду с "лакуной" $\gamma_{n}$ нам надо следить и за убыванием уклонений

$$
\delta_{n}=\mu_{n}-\frac{1}{2}\left(\lambda_{n}^{+}+\lambda_{n}^{-}\right) .
$$

В случае вещественного потенциала $\lambda_{n}^{-} \leqslant \mu_{n} \leqslant \lambda_{n}^{+}$, так что $\left|\delta_{n}\right| \leqslant \gamma_{n}$ и любые оценки $\gamma_{n}$ выполняются для $\delta_{n}$. Однако в случае $(0.1)$

$$
\gamma_{n}=0, \quad \delta_{n} \neq 0
$$

Это привело В. А. Ткаченко [31]-[33] к анализу уклонений $\delta_{n}$. Он нашел в несамосопряженном случае адекватную параметризацию потенциалов в спектральных терминах, подобную рассмотренной В. А. Марченко и И. В. Островским [34], [12] для самосопряженных операторов, и после этого дал условия $C^{\infty}$-гладкости и аналитичности (комплекснозначного) потенциала $v$ в терминах уклонений $\delta_{n}$ и разностей между критическими значениями фунции Ляпунова и $(-1)^{n}$.

Как дальнейшее развитие, Ж.-Ж. Сансюк и В. А. Ткаченко [35] получили утверждения типа Хохштадта: потенииал $v \in L^{2}([0, \pi])$ принадлежит соболевскому пространству $H^{m}, m>0$ - целое, тогда и толъко тогда, когда

$$
\sum\left(\left|\gamma_{n}\right|^{2}+\left|\delta_{n}\right|^{2}\right)\left(1+n^{2 m}\right)<\infty .
$$


6. Говоря об общих классах гладких функций, мы характеризуем гладкость весом $\Omega(n)$ и рассматриваем "соболевское" пространство

$$
H(\Omega)=\left\{v(x)=\sum_{k \in \mathbb{Z}} v_{k} e^{2 i k x}: \sum_{k \in \mathbb{Z}}\left|v_{k}\right|^{2}(\Omega(k))^{2}<\infty\right\}
$$

(подробнее см. п. 3.1).

Родственное пространство последовательностей определяется как

$$
\ell_{\Omega}^{2}=\left\{\xi=\left(\xi_{n}\right)_{0}^{\infty}: \sum\left|\xi_{n}\right|^{2}(\Omega(n))^{2}<\infty\right\} .
$$

В этих терминах основные результаты о гладкости потенциала и скорости убывания "лакун" имеют следующую структуру:

$$
\text { (A) } v \in H(\Omega) \Longleftrightarrow(\mathrm{B}) \quad \gamma \in \ell^{2}(\Omega), \delta \in \ell^{2}(\Omega) \text {. }
$$

Т. Каппелер и Б. С. Митягин [36], [37] дали, при условии (А), процедуру для оценки не только $\gamma$, но и $\delta,-$ некая вариация проекционного метода. Обратные оценки оказались сложнее и тоньше. Но авторы [38] показали, что в случае вещественных потенциалов $v$

$$
\left(\mathrm{B}_{1}\right) \gamma \in \ell^{2}(\Omega) \quad \Longrightarrow \quad(\mathrm{A}) v \in H(\Omega)
$$

и, более того, в [39] показано, что для комплексных потенциалов комбинация условий (В) также влечет (А).

7. При анализе операторов Дирака

$$
L y=i\left(\begin{array}{cc}
1 & 0 \\
0 & -1
\end{array}\right) \frac{d y}{d x}+v(x) y, \quad y=\left(\begin{array}{l}
y_{1} \\
y_{2}
\end{array}\right)
$$

где

$$
v(x)=\left(\begin{array}{cc}
0 & P(x) \\
Q(x) & 0
\end{array}\right), \quad v(x+\pi)=v(x), \quad P, Q \in L^{2}([0, \pi]),
$$

мы следуем той же схеме, основанной на теории возмущений и методе Фурье. Но возникают технические осложнения:

(а) даже свободный оператор $L^{0}$ (с нулевым потенциалом) не является полуограниченным;

(b) его спектр $\operatorname{Sp}\left(L^{0}\right)=2 \mathbb{Z}$, скажем, с периодическими граничными условиями $y_{1}(0)=y_{1}(\pi), y_{2}(0)=y_{2}(\pi)$, неограничен в обе стороны и таков, что расстояние между соседними собственными значениями не уходит в бесконечность;

(c) резольвента $R_{\lambda}^{0}=\left(\lambda-L^{0}\right)^{-1}$, хотя выписывается явно и допускает хорошие оценки, не есть ядерный оператор (т.е. не есть оператор с конечным следом).

Тем не менее, основные результаты о соотношении между лакунами $\gamma_{n}=$ $\lambda_{n}^{+}-\lambda_{n}^{-}$и уклонениями $\delta_{n}=\mu_{n}-\frac{1}{2}\left(\lambda_{n}^{+}+\lambda_{n}^{-}\right), n \in \mathbb{Z}$, корректно определенными по спектрам оператора $L$ с периодическими, антипериодическими граничными условиями и граничными условиями Дирихле (см. детали в пп. 1.1 и 3.1), с одной стороны, и гладкостью потенциала $v$, с другой, доказаны (раздел 4 , в частности, теорема 68) в той же общности.

Если 
(A) $v \in H(\Omega)$, mo

$\left(\mathrm{B}_{1}\right)\left(\gamma_{n}\right) \in \ell^{2}(\mathbb{Z}, \Omega) u$

$\left(\mathrm{B}_{2}\right)\left(\delta_{n}\right) \in \ell^{2}(\mathbb{Z}, \Omega)$.

Обратно, если $v \in L^{2}$, то в самосопряженном случае $($ m.е. $Q=\bar{P})\left(\mathrm{B}_{1}\right) \Rightarrow(\mathrm{A})$ и в общем случае $\left(\mathrm{B}_{1}\right)+\left(\mathrm{B}_{2}\right) \Rightarrow(\mathrm{A})$.

8. В конце каждого раздела мы приводим несколько библиографических замечаний. Они ни в коей мере не имеют своей целью дать обзор огромной литературы, относящейся к затронутым в этой статье (или в соответствующем разделе) темам. Напротив, их цель ограничена - прокомментировать источники некоторых приведенных в статье результатов и используемые в доказательствах технические приемы.

\section{1. Локализация спектра и оценки резольвентных норм}

В этом разделе мы рассматриваем как операторы Хилла-Шрёдингера, так и операторы Дирака в рамках одного и того же подхода, так что мы стараемся использовать одни и те же обозначения, чтобы можно было следовать общей схеме в обоих случаях. Конечно, технические детали могут различаться.

1.1. Основные обозначения и предположения; собственные значения и собственные функции в случае нулевого потенциала. Рассмотрим оператор Хилла-Шрёдингера на отрезке $I=[0, \pi]$

$$
L y=-y^{\prime \prime}+v(x) y
$$

с (комплекснозначным) потенциалом $v \in L^{2}(I)$, так что

$$
v(x)=\sum_{k \in \mathbb{Z}} v_{k} e^{2 i k x} .
$$

Во всей статье мы предполагаем, что

$$
v_{0}=\frac{1}{\pi} \int_{0}^{\pi} v(x) d x=0 .
$$

(Это предположение $v_{0}=0$ не есть ограничение, так как сдвиг потенциала на постоянную сдвигает спектр на ту же постоянную, так что спектральные лакуны остаются без изменений.) Для удобства мы полагаем:

$$
V(j)=\left\{\begin{array}{ll}
v_{j / 2} & \text { для четных } j, \\
0 & \text { для нечетных } j,
\end{array} \quad j \in \mathbb{Z} ;\right.
$$

тогда $(V(j)) \in \ell^{2}(\mathbb{Z})$ и

$$
\|v\|^{2}=\frac{1}{\pi} \int_{0}^{\pi}|v(x)|^{2} d x=\sum_{k \in \mathbb{Z}}\left|v_{k}\right|^{2}=\sum_{j \in 2 \mathbb{Z}}|V(j)|^{2} .
$$

Мы рассматриваем следующие три типа граничных условий (bc): 
(a) периодические $\mathrm{Per}^{+}: y(0)=y(\pi), y^{\prime}(0)=y^{\prime}(\pi)$;

(b) антипериодические $\mathrm{Per}^{-}: y(0)=-y(\pi), y^{\prime}(0)=-y^{\prime}(\pi)$;

(c) Дирихле Dir: $y(0)=0, y(\pi)=0$.

Замкнутый оператор, порожденный $L$, с областью определения

$$
\Delta_{\mathrm{bc}}=\left\{f \in W_{2}^{2}(I): f \in(\mathrm{bc})\right\}
$$

будет соответственно обозначаться через $L_{\mathrm{bc}}$ или $L_{\mathrm{per}}, L_{\mathrm{ap}}, L_{\mathrm{dir}}$. Если $v=0$, мы пишем $L_{\mathrm{bc}}^{0}$ (или просто $L^{0}$ ) или $L_{\mathrm{per}}^{0}, L_{\mathrm{ap}}^{0}, L_{\mathrm{dir}}^{0}$ соответственно. Конечно, легко описать спектры и собственные функции для $L_{\mathrm{bc}}^{0}$. Именно, мы имеем следующее.

(a) $\operatorname{Sp}\left(L_{\text {per }}^{0}\right)=\left\{n^{2}, n=0,2,4, \ldots\right\}$. Он имеет собственные подпространства $E_{n}^{0}=\operatorname{Span}\left\{e^{ \pm i n x}\right\}$ для $n>0$ и $E_{0}^{0}=\{$ const $\}, \operatorname{dim} E_{n}^{0}=2$ для $n>0$, и $\operatorname{dim} E_{0}^{0}=1$.

(b) $\operatorname{Sp}\left(L_{\mathrm{ap}}^{0}\right)=\left\{n^{2}, n=1,3,5, \ldots\right\}$. Его собственными подпространствами являются $E_{n}^{0}=\operatorname{Span}\left\{e^{ \pm i n x}\right\}$, и $\operatorname{dim} E_{n}^{0}=2$.

(c) $\operatorname{Sp}\left(L_{\text {dir }}^{0}\right)=\left\{n^{2}, n \in \mathbb{N}\right\}$. Каждое собственное значение $n^{2}$ - простое; соответствующая нормированная собственная функция есть

$$
s_{n}(x)=\sqrt{2} \sin n x,
$$

так что соответствующее собственное подпространство есть

$$
G_{n}^{0}=\operatorname{Span}\left\{s_{n}\right\} .
$$

Оператор Дирака на отрезке $I=[0, \pi]$ определяется следующим образом:

$$
\begin{gathered}
L y=i\left(\begin{array}{cc}
1 & 0 \\
0 & -1
\end{array}\right) \frac{d y}{d x}+v y \\
v(x)=\left(\begin{array}{cc}
0 & P(x) \\
Q(x) & 0
\end{array}\right), \quad y=\left(\begin{array}{l}
y_{1} \\
y_{2}
\end{array}\right),
\end{gathered}
$$

с $L^{2}$-потенциалом $v$, т.е. $P, Q \in L^{2}(I)$. Пусть

$$
P(x)=\sum_{m \in \mathbb{Z}} P_{m} e^{2 i m \pi x} \quad \text { и } \quad Q(x)=\sum_{m \in \mathbb{Z}} Q_{m} e^{2 i m \pi x}
$$

- разложения Фурье функций $P$ и $Q$. Для удобства мы полагаем:

$$
\begin{gathered}
p(2 m)=P_{m}, \quad q(2 m)=Q_{m}, \\
p(m)=q(m)=0 \text { для нечетных } m ;
\end{gathered}
$$

тогда

$$
\|v\|^{2}=\sum_{m \in \mathbb{Z}}\left(\left|P_{m}\right|^{2}+\left|Q_{m}\right|^{2}\right)=\sum_{k \in 2 \mathbb{Z}}\left(|p(k)|^{2}+|q(k)|^{2}\right) .
$$

Мы снабдим пространство $H^{0}$ векторных $L^{2}(I)$-функций $F=\left(\begin{array}{l}f_{1} \\ f_{2}\end{array}\right)$ скалярным произведением

$$
\langle F, G\rangle=\frac{1}{\pi} \int_{0}^{\pi}\left(f_{1}(x) \overline{g_{1}(x)}+f_{2}(x) \overline{g_{2}(x)}\right) d x .
$$

Мы рассматриваем три типа граничных условий: 
(a) периодические $\operatorname{Per}^{+}: y(0)=y(\pi)$, т.е. $y_{1}(0)=y_{1}(\pi)$ и $y_{2}(0)=y_{2}(\pi)$;

(b) антипериодические $\mathrm{Per}^{-}: y(0)=-y(\pi)$, т.е. $y_{1}(0)=-y_{1}(\pi)$ и $y_{2}(0)=$ $-y_{2}(\pi)$

(с) Дирихле Dir: $y_{1}(0)=y_{2}(0), y_{1}(\pi)=y_{2}(\pi)$.

Соответствующий замкнутый оператор с областью определения

$$
\Delta_{\mathrm{bc}}=\left\{f \in\left(W_{1}^{2}(I)\right)^{2}: F=\left(\begin{array}{l}
f_{1} \\
f_{2}
\end{array}\right) \in(\mathrm{bc})\right\}
$$

будет обозначаться через $L_{\mathrm{bc}}$ или $L_{\mathrm{per}}, L_{\mathrm{ap}}, L_{\mathrm{dir}}$. Если $V=0$, т.е. $P \equiv 0, Q \equiv 0$, то мы пишем $L_{\mathrm{bc}}^{0}$ (или просто $L^{0}$ ) или $L_{\mathrm{per}}^{0}, L_{\mathrm{ap}}^{0}, L_{\mathrm{dir}}^{0}$ соответственно. Конечно, легко описать спектры и собственные функции для $L_{\mathrm{bc}}^{0}$.

(a) $\operatorname{Sp}\left(L_{\text {per }}^{0}\right)=\{n$ четные $\}=2 \mathbb{Z}$; каждое $n \in 2 \mathbb{Z}-$ двойное собственное значение, а соответствующее собственное подпространство есть

$$
E_{n}^{0}=\operatorname{Span}\left\{e_{n}^{1}, e_{n}^{2}\right\}
$$

где

$$
e_{n}^{1}(x)=\left(\begin{array}{c}
e^{-i n x} \\
0
\end{array}\right), \quad e_{n}^{2}(x)=\left(\begin{array}{c}
0 \\
e^{i n x}
\end{array}\right) ;
$$

(b) $\operatorname{Sp}\left(L_{\text {ap }}^{0}\right)=\{n$ нечетные $\}=2 \mathbb{Z}+1$; соответствующие собственные подпространства $E_{n}^{0}$ заданы теми же формулами (1.15) и (1.16), но $n \in 2 \mathbb{Z}+1$;

(c) $\operatorname{Sp}\left(L_{\text {dir }}^{0}\right)=\{n \in \mathbb{Z}\}$; каждое собственное значение $n-$ простое. Соответствующая нормированная собственная функция есть

$$
s_{n}(x)=\frac{1}{\sqrt{2}}\left(e_{n}^{1}+e_{n}^{2}\right), \quad n \in \mathbb{Z},
$$

так что соответствующее (одномерное) собственное подпространство есть

$$
G_{n}^{0}=\operatorname{Span}\left\{s_{n}\right\}
$$

1.2. Формулы для резольвенты возмущенного оператора. Мы рассматриваем оператор

$$
L_{\mathrm{bc}}=L_{\mathrm{bc}}^{0}+V
$$

как возмущение оператора $L_{\mathrm{bc}}^{0}$ и используем стандартные методы теории возмущений [40], [41].

Резольвенту оператора $L \equiv L_{\mathrm{bc}}$ можно представить в разных формах:

$$
R_{\lambda}=(\lambda-L)^{-1}=\left[\left(\lambda-L^{0}\right)-V\right]^{-1}=R_{\lambda}^{0}\left(1-V R_{\lambda}^{0}\right)^{-1}=\left(1-R_{\lambda}^{0} V\right)^{-1} R_{\lambda}^{0},
$$

если

$$
\left\|V R_{\lambda}^{0}\right\|<1 \quad \text { или } \quad\left\|R_{\lambda}^{0} V\right\|<1
$$

соответственно. Часто (1.21) не выполнено для $\lambda$, которые могут быть важны. Но так как

$$
(1-T)^{-1}=(1+T)\left(1-T^{2}\right)^{-1},
$$

то, если все операторы в этой строке вполне определены, или во всяком случае, если
(а) $\left\|V R_{\lambda}^{0} V R_{\lambda}^{0}\right\|<1 \quad$ и
(b) $\left\|V R_{\lambda}^{0}\right\|<\infty$ 
или если

$$
\text { (a) }\left\|R_{\lambda}^{0} V R_{\lambda}^{0} V\right\|<1 \quad \text { и } \quad \text { (b) }\left\|R_{\lambda}^{0} V\right\|<\infty \text {, }
$$

можно воспользоваться следующими тождествами:

$$
R_{\lambda}=R_{\lambda}^{0}\left(1+V R_{\lambda}^{0}\right)\left(1-V R_{\lambda}^{0} V R_{\lambda}^{0}\right)^{-1}
$$

или

$$
R_{\lambda}=\left(1+R_{\lambda}^{0} V\right)\left(1-R_{\lambda}^{0} V R_{\lambda}^{0} V\right)^{-1} R_{\lambda}^{0} .
$$

Если (1.23) выполнено в сильной форме

$$
\left\|V R_{\lambda}^{0} V R_{\lambda}^{0}\right\| \leqslant 1 / 2
$$

то мы имеем следующую оценку для нормы резольвенты $R_{\lambda}$ :

$$
\left\|R_{\lambda}\right\| \leqslant 2\left\|R_{\lambda}^{0}\right\|\left(1+\left\|V R_{\lambda}^{0}\right\|\right) .
$$

В силу (1.20) отклонение резольвенты $R_{\lambda}$ от $R_{\lambda}^{0}$ вычисляется как

$$
R_{\lambda}-R_{\lambda}^{0}=R_{\lambda}^{0} V R_{\lambda}^{0}\left(1-R_{\lambda}^{0} V\right)^{-1}
$$

или

$$
R_{\lambda}-R_{\lambda}^{0}=\left(1-V R_{\lambda}^{0}\right)^{-1} R_{\lambda}^{0} V R_{\lambda}^{0} .
$$

Конечно, мы используем (1.22), если это возможно.

Оператор $V$ умножения на функцию $v(x)$ в случае Хилла-Шрёдингера или на матричную функцию $v(x)$ в случае Дирака неограничен в $H^{0}=L^{2}(I)$, но он действует как

$$
\begin{gathered}
V: L^{\infty}(I) \rightarrow L^{2}(I) \quad \text { и } \quad V: L^{2}(I) \rightarrow L^{1}(I), \\
\|V\|_{\infty \rightarrow 2}=\|V\|_{2 \rightarrow 1}=\|v\| .
\end{gathered}
$$

Это приводит к следующим неравенствам:

$$
\begin{gathered}
\left\|V R_{\lambda}^{0}\right\|_{2 \rightarrow 2} \leqslant\|v\|\left\|R_{\lambda}^{0}: L^{2} \rightarrow L^{\infty}\right\|, \\
\left\|R_{\lambda}^{0} V\right\|_{2 \rightarrow 2} \leqslant\left\|R_{\lambda}^{0}: L^{1} \rightarrow L^{2}\right\|\|v\|=\|v\|\left\|R_{\bar{\lambda}}^{0}: L^{2} \rightarrow L^{\infty}\right\|
\end{gathered}
$$

и

$$
\begin{gathered}
\left\|V R_{\lambda}^{0} V R_{\lambda}^{0}\right\| \leqslant\|v\|\left\|R_{\lambda}^{0} V R_{\lambda}^{0}\right\|_{2 \rightarrow \infty} \\
\left\|R_{\lambda}^{0} V R_{\lambda}^{0}\right\| \leqslant\left\|R_{\lambda}^{0} V R_{\lambda}^{0}\right\|_{1 \rightarrow 2}\|v\|=\|v\|\left\|R_{\bar{\lambda}}^{0} V^{*} R_{\bar{\lambda}}^{0}\right\|_{2 \rightarrow \infty} .
\end{gathered}
$$

Мы пишем равенства справа в (1.34) и (1.36), поскольку нормы сопряженных операторов равны. Формулы (1.35) и (1.36) показывают, что мы сможем получить (1.27) или

$$
\left\|R_{\lambda}^{0} V R_{\lambda}^{0} V\right\| \leqslant 1 / 2
$$

если $\left\|R_{\lambda}^{0} V R_{\lambda}^{0}\right\|_{2 \rightarrow \infty}$ достаточно мало. Если бы (1.23)(b) или (1.24)(b) было выполнено, то это гарантировало бы, что $R_{\lambda}$ вполне определен соотношением (1.25) или (1.26) и $\lambda$ - регулярная точка, т.е. $\lambda \notin \operatorname{Sp}\left(L_{\mathrm{bc}}\right)$. 
Более того, хорошие оценки для

$$
\left\|R_{\lambda}^{0} V R_{\lambda}^{0}: L^{2} \rightarrow L^{\infty}\right\|
$$

могут быть использованы для того, чтобы оценивать уклонение проекторов $P_{\mathrm{bc}}$ и $P_{\mathrm{bc}}^{0}$ на замкнутых инвариантных подпространствах операторов $L_{\mathrm{bc}}$ и $L_{\mathrm{bc}}^{0}$. Более точно, если $C=\partial D$ - гладкая кривая, являющаяся границей области $D \subset \mathbb{C}$, и $C \cap\left(\operatorname{Sp}\left(L_{\mathrm{bc}}\right) \cup \operatorname{Sp}\left(L_{\mathrm{bc}}^{0}\right)\right)=\varnothing$, то проекторы Коши-Рисса

$$
P_{\mathrm{bc}}(D)=\frac{1}{2 \pi i} \int_{C} R_{\lambda} d \lambda, \quad P_{\mathrm{bc}}^{0}(D)=\frac{1}{2 \pi i} \int_{C} R_{\lambda}^{0} d \lambda
$$

вполне определены и

$$
P_{\mathrm{bc}}-P_{\mathrm{bc}}^{0}=\frac{1}{2 \pi i} \int_{C}\left(R_{\lambda}-R_{\lambda}^{0}\right) d \lambda .
$$

В силу (1.29), (1.30) и (1.27) мы имеем:

$$
\begin{aligned}
\left\|P_{\mathrm{bc}}-P_{\mathrm{bc}}^{0}\right\|_{2 \rightarrow \gamma} & =\frac{1}{2 \pi}\left\|\int_{C}\left(R_{\lambda}-R_{\lambda}^{0}\right) d \lambda\right\|_{2 \rightarrow \gamma} \\
& \leqslant \frac{1}{2 \pi} \int_{C}\left\|R_{\lambda}^{0} V R_{\lambda}^{0}\right\|_{2 \rightarrow \gamma}|d \lambda|, \quad \gamma=2 \text { или } \infty .
\end{aligned}
$$

Это замечание, вместе с соотношениями (1.35) и (1.36), показывает, что оценки норм $\left\|R_{\lambda}^{0} V R_{\lambda}^{0}\right\|_{2 \rightarrow \gamma}$ могут быть критическими в нашем анализе. Когда, т.е. для каких $\lambda$, мы можем иметь хорошие оценки?

1.3. Матричное представление операторов умножения. В предыдущем пункте была дана серия основных формул теории возмущений. Но они имеют смысл, только если у нас есть хорошие оценки, чтобы гарантировать (1.21), или (1.23) и (1.24). В следующих пунктах мы докажем подходящие неравенства для норм резольвент, чтобы сделать возможным применение построений п. 1.2. Для анализа операторов $L_{\mathrm{bc}}$ мы используем их матричное представление относительно соответствующего ортонормированного базиса, построенного из собственных векторов свободного оператора $L_{\mathrm{bc}}^{0}$ в $H^{0}=L^{2}(I)$. Пусть $\Gamma_{\mathrm{bc}}$ будет соответствующим множеством индексов или в случае Хилла-Шрёдингера, или в случае Дирака.

В первом случае мы имеем:

$$
\Gamma=\Gamma_{\mathrm{bc}}= \begin{cases}2 \mathbb{Z} & \text { для } \mathrm{Per}^{+}, \\ 2 \mathbb{Z}+1 & \text { для } \mathrm{Per}^{-}, \\ \mathbb{N} & \text { для } \mathrm{Dir}\end{cases}
$$

(см. п. 1.1).

Матричные представления оператора умножения $V: f \rightarrow v f$ не так уж просты в случае граничных условий Дирихле. Поэтому нам нужны для случая Хилла-Шрёдингера и для случая Дирака следующие леммы. 
Лемма 1. Пусть $\left(g_{p}^{0}\right)_{p \geqslant 1}$ - ортонормированный базис собственных функuцй оператора $L_{\mathrm{dir}}^{0}$ в случае Хилла-Шрёдингера. Тогда

$$
\left\langle V g_{j}^{0}, g_{k}^{0}\right\rangle=u_{1}(j+k)+u_{2}(j-k)
$$

¿de

$$
u_{\tau}=H_{\tau} v, \quad \tau=1,2,
$$

- $\ell^{2}(\mathbb{Z})$-последовательности, а $H_{\tau}: \ell^{2}(\mathbb{N}) \rightarrow \ell^{2}(\mathbb{Z})$ - ограниченные операторы такие, что

$$
\left\|H_{\tau}\right\| \leqslant 10, \quad \tau=1,2 .
$$

ДокАЗАтЕльство. В силу (1.7) мы имеем:

$$
V_{j k}=\sum_{n \in 2 \mathbb{Z}} V(n) E_{j k}^{n}
$$

где

$$
E^{n}: f \rightarrow e^{i n x} f(x), \quad n \in \mathbb{Z}
$$

и

$$
E_{j k}^{n}=\frac{2}{\pi} \int_{0}^{\pi} e^{i n x} \sin j x \sin k x d x, \quad j, k \geqslant 1 .
$$

Если $j+k$ четно, то

$$
E_{j k}^{n}= \begin{cases}0, & n \neq \pm j \pm k \\ -1 / 2, & n= \pm(j+k) \\ 1 / 2, & n= \pm(j-k)\end{cases}
$$

при $j-k \neq 0$ и

$$
E_{j j}^{n}= \begin{cases}1, & n=0 \\ -1 / 2, & n= \pm 2 j \\ 0, & n \neq 0, \pm 2 j\end{cases}
$$

Если же $j-k$ нечетно, то

$$
i E_{j k}^{n}=\frac{1}{\pi}\left[\frac{1}{n+(j+k)}+\frac{1}{n-(j+k)}-\frac{1}{n+(j-k)}-\frac{1}{n-(j-k)}\right] .
$$

Ввиду (1.46), (1.49) и (1.50) мы имеем: для четных $j+k$

$$
2 V_{j k}=V(j-k)+V(k-j)-V(j+k)-V(-j-k),
$$

а для $j+k$ нечетных

$$
\pi i V_{j k}=w(j+k)+w(-j-k)-w(j-k)-w(-j+k),
$$

где

$$
w(m)=\sum_{n \text { четные }} \frac{v(n)}{n-m}, \quad m \text { - нечетные. }
$$

Оператор $H: v \rightarrow w$ в (1.54) ограничен (как преобразование Гильберта из $\ell^{2}(2 \mathbb{Z})$ в $\left.\ell^{2}(2 \mathbb{Z}+1)\right)$, и $\|H\| \leqslant 2$. 
Более того, (1.53) и (1.52) показывают, что

$$
i V_{j k}=w_{1}(j+k)-w_{1}(j-k),
$$

где

$$
w_{1}(m)= \begin{cases}-(V(m)+V(-m)) /(2 i), & m \text { - четные } \\ (w(m)+w(-m)) / \pi, & m \text { - нечетные. }\end{cases}
$$

Это завершает доказательство леммы 1.

Лемма 2. Пусть $\left(g_{p}^{0}\right)_{p \geqslant 1}$ - ортонормированный базис собственных функuий оператора $L_{\mathrm{dir}}^{0}$ в случае Дирака. Тогда

$$
\left\langle V g_{j}^{0}, g_{k}^{0}\right\rangle=w(j+k), \quad j, k \geqslant 1,
$$

¿əe

$$
w=H v \in \ell^{2}(\mathbb{Z})
$$

и $H: \ell^{2}(\mathbb{N}) \rightarrow \ell^{2}(\mathbb{Z})$ - такой ограниченный оператор, что

$$
\|H\| \leqslant 6 .
$$

ДокаЗАТЕЛЬство. Рассмотрим

$$
\begin{gathered}
v=\left(\begin{array}{ll}
0 & 0 \\
Q & 0
\end{array}\right), \\
Q(x)=\sum_{k \in \mathbb{Z}} q(k) e^{i k x} \quad \text { как в (1.13). }
\end{gathered}
$$

Конечно, матричная функция $v=\left(\begin{array}{ll}0 & P \\ 0 & 0\end{array}\right)$, где $P$ как в $(1.11),(1.13)$, может быть проанализирована тем же способом. Поэтому мы рассматриваем только (1.60), так что

$$
\begin{gathered}
v=\sum_{n \in 2 \mathbb{Z}} q(n) E^{n}, \quad \text { где } \quad E^{n}=\left(\begin{array}{cc}
0 & 0 \\
e^{i n x} & 0
\end{array}\right), \\
E^{n}:\left(\begin{array}{l}
f_{1} \\
f_{2}
\end{array}\right) \rightarrow\left(\begin{array}{c}
0 \\
e^{i n x} f_{1}(x)
\end{array}\right), \quad n \in 2 \mathbb{Z} .
\end{gathered}
$$

Тогда

$$
\begin{aligned}
E_{j k}^{n} & =\frac{1}{\pi} \int_{0}^{\pi} e^{i n x} e^{-i j x} \overline{e^{i k x}} d x \\
& = \begin{cases}1, & \text { если } j+k \text { четно и равно } n, \\
0, & \text { если } j+k \text { четно и не равно } n, \\
-\frac{2}{i \pi} \cdot \frac{1}{n-(j+k)}, & \text { если } j+k \text { нечетно. }\end{cases}
\end{aligned}
$$

Ввиду (1.61)

$$
V_{j k}= \begin{cases}q(j+k), & \text { если } j+k \text { четно, } \\ q_{1}(j+k), & \text { если } j+k \text { нечетно, }\end{cases}
$$


где

$$
q_{1}(m)=\frac{2 i}{\pi} \sum_{n \text { четные }} \frac{q(n)}{n-m},
$$

так что это - матрица Ганкеля

$$
\{w(j+k)\} \quad \text { c } \quad w \in \ell^{2}(\mathbb{Z}) .
$$

Лемма 2 доказана.

Более простое утверждение - но оно тоже должно быть упомянуто - дает ганкелевское матричное представление оператора $V$ и для $\mathrm{bc}=\mathrm{Per}^{ \pm}$. Мы обозначим через $\mathscr{H}^{1}$ и $\mathscr{H}^{2}$ подпространства порожденные соответственно системами $\left\{e_{k}^{\tau}, k \in \Gamma_{\mathrm{bc}}\right\}, \tau=1,2$, если $\mathrm{bc}=\operatorname{Per}^{ \pm}$(см. (1.16)). Если $v$ имеет

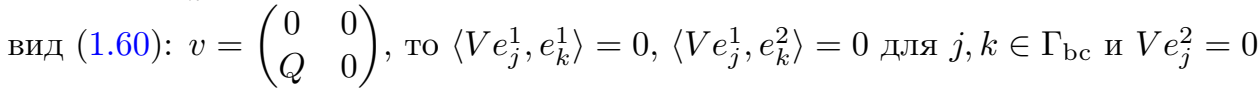
для всех $j$. Следовательно,

$$
V \sim\left(\begin{array}{cc}
0 & 0 \\
V^{21} & 0
\end{array}\right), \quad \text { где } \quad V^{21}: \mathscr{H}^{1} \rightarrow \mathscr{H}^{2},
$$

и $V^{21}$ имеет ганкелевское матричное представление, именно, $\left(V^{21}\right)_{j k}=q(j+k)$.

Аналогичным образом можно показать, что если $v$ имеет вид $v=\left(\begin{array}{ll}0 & P \\ 0 & 0\end{array}\right)$, то $V \sim\left(\begin{array}{cc}0 & V^{12} \\ 0 & 0\end{array}\right)$, где $V^{12}: \mathscr{H}^{2} \rightarrow \mathscr{H}^{1}$ и $\left(V^{12}\right)_{j k}=p(-j-k)$.

1.4. Оценки норм $\left\|R_{\lambda}^{0} V R_{\lambda}^{0}\right\|$. Для всех граничных условий $\mathrm{bc}=\mathrm{Per}^{ \pm}, \mathrm{Dir}$ операторы $L_{\mathrm{bc}}^{0}$ и $R_{\lambda}^{0}=\left(\lambda-L_{\mathrm{bc}}^{0}\right)^{-1}$ имеют диагональные матричные представления

$$
\left(k^{2} \delta_{j k}\right)_{j, k \in \Gamma_{\mathrm{bc}}}, \quad\left(\left(\lambda-k^{2}\right)^{-1} \delta_{j k}\right)_{j, k \in \Gamma_{\mathrm{bc}}}
$$

относительно соответствующего ортонормированного базиса (см. п. 1.1). Эти базисы равномерно ограничены в $L^{\infty}$; более точно,

$$
\sup _{n \in \Gamma_{\mathrm{bc}}} \sup _{x \in I}\left|u_{n}(x)\right| \leqslant 2
$$

для всех шести ортонормированных базисов, описанных в п. 1.1. Таким образом, мы имеем следующее элементарное неравенство:

$$
\left\|\sum \xi_{n} u_{n}(x)\right\|_{\infty} \leqslant 2 \sum\left|\xi_{n}\right| \equiv 2\left\|\xi \mid \ell^{1}\left(\Gamma_{\mathrm{bc}}\right)\right\| .
$$

Поэтому если

$$
a=\left(A_{j k}\right)_{j, k \in \Gamma}
$$

- матричное представление оператора $A$, то мы можем оценить его норму как оператора из $L^{2}$ в $L^{\infty}$ следующим образом:

$$
\left\|A: L^{2} \rightarrow L^{\infty}\right\| \leqslant 2 \alpha
$$


где

$\alpha:=\sup \left\{\left|\sum_{j, k} A_{j k} x_{k} y_{j}\right|:\|x\|_{\ell^{2}}=1,\|y\|_{\ell^{\infty}}=1\right\}=\left\|a: \ell^{2} \rightarrow \ell^{1}\right\|=\left\|a^{t}: \ell^{\infty} \rightarrow \ell^{2}\right\|$,

$a^{t}$ - транспонированная матрица. Элементарные неравенства показывают, что

$$
\alpha \leqslant \sum_{j}\left(\sum_{k}\left|A_{j k}\right|^{2}\right)^{1 / 2} \text { и } \alpha \leqslant\left(\sum_{k}\left(\sum_{j}\left|A_{j k}\right|\right)^{2}\right)^{1 / 2} \text {. }
$$

Конечно, в дальнейшем мы много раз используем нормы Гильберта-Шмидта:

$$
\left\|A: L^{2} \rightarrow L^{2}\right\|=\left\|a: \ell^{2} \rightarrow \ell^{2}\right\| \leqslant\|A\|_{\mathrm{HS}} \equiv\left(\sum_{j, k}\left|A_{j k}\right|^{2}\right)^{1 / 2} .
$$

Если $a$ - диагональная матрица $a=\left(a_{k} \delta_{j k}\right)$, то (1.72) влечет за собой, что

$$
\left\|A: L^{2} \rightarrow L^{\infty}\right\| \leqslant 2\left\|a: \ell^{2} \rightarrow \ell^{1}\right\| \leqslant 2\left(\sum\left|a_{k}\right|^{2}\right)^{1 / 2}
$$

в этом случае

$$
\left\|A: L^{2} \rightarrow L^{2}\right\|=\left\|a: \ell^{2} \rightarrow \ell^{2}\right\|=\sup _{k \in \Gamma_{\mathrm{bc}}}\left|\alpha_{k}\right| .
$$

ЗАмечАниЕ 3. Функции $u_{n}(x)$ непрерывны. Операторы $A$, построенные в (1.70)-(1.74), действуют из $L^{2}$ в пространство непрерывных функций $C([0, \pi])$, хотя часто мы пишем $L^{\infty}$.

Теперь мы готовы дать оценки норм операторов, упомянутых в п. 1.2.

Важный пример, где мы используем неравенства (1.72), относится к матричным представлениям (при различных граничных условиях) оператора $R_{\lambda}^{0} V R_{\lambda}^{0}$ в случае Дирака. Лемма 2 показывает, что каждое из этих матричных представлений может быть дано в виде

$$
a=\left(\frac{w(j+k)}{(\lambda-j)(\lambda-k)}\right), \quad j, k \in \Gamma_{\mathrm{bc}}
$$

где $w$ это $\ell^{2}$-последовательность.

Положим для каждой $\ell^{2}$-последовательности $f$ (множество индексов может быть $\mathbb{Z}$, или $2 \mathbb{Z}$, или $2 \mathbb{Z}+1$ )

$$
\mathscr{E}_{m}(f)=\left(\sum_{|k| \geqslant m}\left|f_{k}\right|^{2}\right)^{1 / 2}, \quad m>0
$$

ЛЕмма 4. При принятых выше обозначениях, если $n \in \mathbb{Z}, n \neq 0 u$

(a) $w \in \ell^{2}(\mathbb{Z}), j, k, n \in \mathbb{Z} u \lambda=n+x+i t$, где $|x| \leqslant 1 / 2$, или

(b) $w \in \ell^{2}(2 \mathbb{Z}), j, k \in n+2 \mathbb{Z} u \lambda=n+x+i t$, где $|x| \leqslant 1$, 
mo

$$
\begin{aligned}
\Sigma(n) & :=\sum_{k}\left(\sum_{j}\left|\frac{w(j+k)}{(\lambda-j)(\lambda-k)}\right|\right)^{2} \\
& \leqslant C\left(\frac{|w(2 n)|^{2}}{r^{4}}+\frac{\|w\|^{2}}{|n|+r}\left(\frac{1}{r^{2}}+\frac{1}{1+r}\right)+\frac{(\mathscr{E}|n| / 2(w))^{2}}{1+r}\left(\frac{1}{r^{2}}+\frac{1}{1+r}\right)\right),
\end{aligned}
$$

где $r=|\lambda-n|=\sqrt{x^{2}+t^{2}}$ u $C>0-$ абсолютная постоянная.

ДокАзАтельство. Доказательство в случаях (a) и (b) одно и то же, поэтому мы рассмотрим только случай (а). Имеем:

$$
\Sigma(n)=\sum_{k} \frac{1}{|\lambda-k|^{2}}(\sigma(k))^{2},
$$

где

$$
\sigma(k)=\sum_{j} \frac{|w(j+k)|}{|\lambda-j|} .
$$

Чтобы оценить (1.80), воспользуемся неравенством

$$
\frac{1}{|\lambda-j|} \leqslant \frac{3}{|n-j|+r}, \quad \text { если } \quad|x| \leqslant \frac{1}{2}, \quad r=\sqrt{x^{2}+t^{2}}, \quad j \neq n .
$$

Действительно, $\operatorname{Re}(\lambda-j)=n-j+x$ при $|x| \leqslant 1 / 2$, и, следовательно,

$$
|\lambda-x| \geqslant|n-j+x| \geqslant|n-j|-1 / 2 \geqslant|n-j| / 2, \quad j \neq n .
$$

С другой стороны, так как $|\operatorname{Re}(\lambda-j)| \geqslant|n-j|-1 / 2 \geqslant 1 / 2$ при $j \neq n$, то мы имеем

$$
|\lambda-j| \geqslant|1 / 2+i t| \geqslant|x+i t|=r
$$

так что $3|\lambda-j| \geqslant|n-j|+r$, т.е. (1.81) выполнено.

В дальнейшем мы также используем следующее элементарное неравенство.

Лемма 5. Для любых двух $\ell^{2}(\mathbb{Z})$-последовательностей $(d(j)) u(b(j))$ мъь имеем:

$$
\left|\sum_{j} d(j) b(2 n-j)\right|=\left|\sum_{j} d(j+n) b(n-j)\right| \leqslant\|d\| \mathscr{E}_{|n|}(b)+\|b\| \mathscr{E}_{|n|}(d) .
$$

ДокАЗАТЕЛЬСтво. Действительно,

$$
\sum_{j} d(j+n) b(n-j)=\sum_{j \geqslant 0} \cdots+\sum_{j<0} \cdots,
$$

и по неравенству Коши мы имеем:

$$
\left|\sum_{j \geqslant 0} \cdots\right| \leqslant\left(\sum_{j \geqslant 0}|d(j+n)|^{2}\right)^{1 / 2}\|b\| \leqslant\|b\| \mathscr{E}_{|n|}(d) .
$$


Аналогичным образом получается, что

$$
\left|\sum_{j<0} \cdots\right| \leqslant\|d\|\left(\sum_{j<0}|b(n-j)|^{2}\right)^{1 / 2} \leqslant\|d\| \mathscr{E}_{|n|}(b) ;
$$

это завершает доказательство.

В силу (1.81) мы имеем:

$$
\sigma(k)=\frac{|w(n+k)|}{|\lambda-n|}+\sum_{j \neq n} \frac{|w(j+k)|}{|\lambda-j|} \leqslant \frac{|w(n+k)|}{r}+3 \sum_{j \neq n} \frac{|w(n+k)|}{|n-j|+r} .
$$

По лемме 5 , если $d(j)=w(j+k-n)$ и

$$
b(j)=\frac{1}{|j|+r} \quad \text { для } \quad j \neq 0, \quad b(0)=0,
$$

то

$$
\sigma(k) \leqslant \frac{|w(n+k)|}{r}+3\left(\|d\| \mathscr{E}_{|n|}(b)+\mathscr{E}_{|n|}(d)\|b\|\right)
$$

Мы имеем:

$$
\mathscr{E}_{|n|}(d) \leqslant\|d\|=\|w\|, \quad \mathscr{E}_{|n|}(d) \leqslant \mathscr{E}_{|n| / 2}(w), \quad \text { если } \quad|k-n|<|n| / 2,
$$

И

$$
\left(\mathscr{E}_{|n|}(b)\right)^{2}=\frac{2}{(|n|+r)^{2}}+\sum_{j=n+1}^{\infty} \frac{2}{(j+r)^{2}} \leqslant \frac{2}{|n|+r}+\int_{|n|}^{\infty} \frac{2}{(s+r)^{2}} d s=\frac{4}{|n|+r},
$$

так что

$$
\mathscr{E}_{|n|}(b) \leqslant \frac{2}{\sqrt{|n|+r}}, \quad\|b\|=\mathscr{E}_{1}(b) \leqslant \frac{2}{\sqrt{1+r}} .
$$

Таким образом, (1.83) и (1.85)-(1.86) влекут за собой, что

$$
\sigma(k) \leqslant \begin{cases}\frac{|w(n+k)|}{r}+\frac{6\|w\|}{\sqrt{|n|+r}}+\frac{6 \mathscr{E}_{|n| / 2}(w)}{\sqrt{1+r}}, & \text { если }|k-n|<\frac{|n|}{2}, \\ \frac{|w(n+k)|}{r}+\frac{12|| w \mid}{\sqrt{1+r}}, & \text { если }|k-n| \geqslant \frac{|n|}{2} .\end{cases}
$$

Теперь оценим сумму $\Sigma(n)$ :

$$
\Sigma(n)=\sum_{k} \frac{(\sigma(k))^{2}}{|\lambda-k|^{2}}=\frac{(\sigma(n))^{2}}{r^{2}}+\sum_{0<|k-n|<|n| / 2} \cdots+\sum_{|k-n| \geqslant|n| / 2} \cdots
$$

Ввиду (1.87), с некоторой абсолютной постоянной $C>0$ мы имеем:

$$
\frac{(\sigma(n))^{2}}{r^{2}} \leqslant C\left(\frac{|w(2 n)|^{2}}{r^{4}}+\frac{\|w\|^{2}}{r^{2}(|n|+r)}+\frac{\left(\mathscr{E}_{|n| / 2}(w)\right)^{2}}{r^{2}(1+r)}\right)
$$




$$
\begin{aligned}
& \sum_{0<|k-n|<|n| / 2} \leqslant \sum_{0<|k-n|<|n| / 2} \frac{9(\sigma(k))^{2}}{(|n-k|+r)^{2}} \\
& \leqslant C\left(\sum_{0<|k-n|<|n| / 2} \frac{|w(n+k)|^{2}}{r^{2}(|n-k|+r)^{2}}\right. \\
& \left.+\left(\frac{\|w\|^{2}}{|n|+r}+\frac{\left(\mathscr{E}_{|n| / 2}(w)\right)^{2}}{1+r}\right) \sum_{k \neq n} \frac{1}{(|n-k|+r)^{2}}\right) \\
& \leqslant C\left(\frac{\left(\mathscr{E}_{|n| / 2}(w)\right)^{2}}{r^{2}(1+r)}+\left(\frac{\|w\|^{2}}{|n|+r}+\frac{(\mathscr{E}|n| / 2(w))^{2}}{1+r}\right) \frac{1}{1+r}\right) \\
& \sum_{|k-n| \geqslant|n| / 2} \leqslant \sum_{|k-n| \geqslant|n| / 2} \frac{9(\sigma(k))^{2}}{(|n-k|+r)^{2}} \\
& \leqslant C\left(\frac{1}{r^{2}} \sum_{|k-n| \geqslant|n| / 2} \frac{|w(n+k)|^{2}}{(|n-k|+r)^{2}}+\frac{\|w\|^{2}}{1+r} \sum_{|k-n| \geqslant|n| / 2} \frac{1}{(|n-k|+r)^{2}}\right) \\
& \leqslant C\left(\frac{\|w\|^{2}}{r^{2}(|n|+r)^{2}}+\frac{\|w\|^{2}}{(1+r)(|n|+r)}\right) \text {. }
\end{aligned}
$$

Из соотношений (1.88)-(1.91) вытекает (1.78). Это завершает доказательство леммы 4.

Нам будет также нужна следующая модификация леммы 4; ее доказательство то же самое.

ЛЕмма 6. При предположениях леммы 4 для всех $n \in \mathbb{Z}$ мы имеем:

$$
\Sigma^{*}(n):=\sum_{k \neq n}\left(\sum_{j \neq n} \frac{w(j+k)}{|n-j||n-k|}\right)^{2} \leqslant C\left(\frac{\|w\|^{2}}{|n|+1}+\left(\mathscr{E}_{|n|}(w)\right)^{2}\right),
$$

где $C>0-$ абсолютная постоянная.

\section{5. Оценки резольвентных норм и локализация спектра оператора} Хилла-Шрёдингера; уклонение собственных подпространств свободного и возмущенного операторов. Резольвента $R_{\lambda}^{0}$ - это диагональный оператор, так что в случае Хилла-Шрёдингера

$$
\begin{gathered}
\left\|R_{\lambda}^{0}\right\|=\max _{k \in \Gamma_{\mathrm{bc}}} \frac{1}{\left|\lambda-k^{2}\right|}, \\
\left\|R_{\lambda}^{0}\right\|_{2 \rightarrow \infty} \leqslant\left\|r_{\lambda}^{0}: \ell^{2} \rightarrow \ell^{1}\right\|=\sqrt{b(\lambda)},
\end{gathered}
$$

где

$$
b(\lambda)=\sum_{k \in \Gamma_{\mathrm{bc}}} \frac{1}{\left|\lambda-k^{2}\right|^{2}}
$$

Ввиду (1.33) мы имеем:

$$
\left\|V R_{\lambda}^{0}\right\| \leqslant\|v\| \sqrt{b(\lambda)}, \quad \lambda \notin \operatorname{Sp}\left(L_{\mathrm{bc}}^{0}\right) .
$$


Тот же довод показывает, что

$$
\left\|R_{\lambda}^{0} V\right\|=\|v\|\left\|R_{\lambda}^{0}: L^{1} \rightarrow L^{2}\right\| \leqslant \sqrt{b(\lambda)}\|v\|, \quad \lambda \notin \operatorname{Sp}\left(L_{\mathrm{bc}}^{0}\right) .
$$

Чтобы оценить $b(\lambda)$, или другие функции того же типа, которые появятся позже, разобьем комплексную плоскость на полосы:

$$
\mathbb{C}=\bigcup_{n \text { четные }} H_{n}=\bigcup_{n \text { нечетные }} H_{n} .
$$

Сначала рассмотрим граничные условия $\mathrm{Per}^{ \pm}$; тогда

$$
\begin{aligned}
& H_{0}=\{\lambda \in \mathbb{C}: \operatorname{Re} \lambda \leqslant 1\}, \quad H_{1}=\{\lambda \in \mathbb{C}: \operatorname{Re} \lambda \leqslant 4\}, \\
& H_{n}=\left\{\lambda \in \mathbb{C}:(n-1)^{2} \leqslant \operatorname{Re} \lambda \leqslant(n+1)^{2}\right\}, \quad n \geqslant 2 .
\end{aligned}
$$

ЛЕмма 7. Пусть $\lambda \in H_{n}, \lambda=n^{2}+z, z=x+i t$. Eсли $\operatorname{Re} \lambda \geqslant 0$, mо для всех $n \geqslant 1$

$$
b_{n}(\lambda):=\sum_{\substack{k \neq \pm n \\ k \in n+2 \mathbb{Z}}} \frac{1}{\left|\lambda-k^{2}\right|^{2}} \sim g(n, t),
$$

¿əe

$$
g(n, t)= \begin{cases}n^{-2}, & 0 \leqslant|t| \leqslant n \\ (n|t|)^{-1}, & n \leqslant|t| \leqslant n^{2} \\ |t|^{-3 / 2}, & n^{2} \leqslant|t|\end{cases}
$$

Eсли $\operatorname{Re} \lambda \leqslant 0, m o$

$$
b_{0}(\lambda):=\sum_{k \neq 0} \frac{1}{\left|\lambda-k^{2}\right|^{2}} \sim \min \left(1,|\lambda|^{3 / 2}\right) .
$$

(Запись $u \sim v$ означает, что существует такая абсолютная постоянная $T>0$, что $T^{-1} u \leqslant v \leqslant T u$.)

В случае граничного условия Дирихле $\Gamma_{\mathrm{bc}}=\mathbb{N}$, так что нам надо подправить выбор в (1.99) и (1.100). Пусть

$$
\begin{gathered}
H_{0}=\{\lambda \in \mathbb{C}: \operatorname{Re} \lambda \leqslant 1 / 2\}, \quad H_{1}=\{\lambda \in \mathbb{C}: 1 / 2 \leqslant \operatorname{Re} \lambda \leqslant 3\}, \\
H_{n}=\left\{\lambda \in \mathbb{C}: n^{2}-n \leqslant \operatorname{Re} \lambda \leqslant n^{2}+n\right\}, \quad n \geqslant 2 .
\end{gathered}
$$

Лемма 8. Если $n \geqslant 1, \lambda \in H_{n}$, mo

$$
c_{n}(\lambda):=\sum_{k \in \mathbb{N} \backslash\{n\}} \frac{1}{\left|\lambda-k^{2}\right|^{2}} \sim g(n, t),
$$

где $g(n, t)$ определено в (1.102). Если $\lambda \in H_{0}$, то

$$
c_{0}(\lambda):=\sum_{k \neq 0} \frac{1}{\left|\lambda-k^{2}\right|^{2}} \sim \min \left(1,|\lambda|^{3 / 2}\right) .
$$


Так как

$$
\left|\lambda-k^{2}\right|^{2} \sim\left(n^{2}-k^{2}\right)^{2}+t^{2} \quad \text { для } \quad k \neq \pm n, \quad \lambda=n^{2}+x+i t \in H_{n},
$$

то доказательства обеих лемм 7 и 8 выводятся из леммы 79 (см. дополнение).

Леммы 7 и 8 могут быть использованы, чтобы получить хорошие оценки норм (1.21). Действительно, если с обозначениями (1.104), (1.105) $\lambda=n^{2}+x+$ it $\in H_{n}, n \geqslant 2$ (или $n \geqslant 1$ в случае граничных условий Дирихле), то

$$
\max \left(\left\|V R_{\lambda}^{0}\right\|_{2 \rightarrow 2},\left\|R_{\lambda}^{0} V\right\|_{2 \rightarrow 2}\right) \leqslant C\|v\|\left(\frac{1}{|x|+|t|}+(g(n, t))^{1 / 2}\right),
$$

а если $\lambda \in H_{0}$ или $H_{1}$ (или $H_{0}$ для граничных условий Дирихле), то

$$
\max \left(\left\|V R_{\lambda}^{0}\right\|_{2 \rightarrow 2},\left\|R_{\lambda}^{0} V\right\|_{2 \rightarrow 2}\right) \leqslant C\|v\|\left((|x-n|+|t|)^{-1}+(|x-n|+|t|)^{-3 / 4}\right),
$$

где $C$ - абсолютная постоянная.

Пусть

$$
D(n ; r)=\left\{\lambda \in \mathbb{C}:\left|\lambda-n^{2}\right| \leqslant r\right\} .
$$

Теорема 9 (локализация спектра операторов Хилла-Шрёдингера). Пусть $L_{\mathrm{bc}}$ - оператор (1.1) u bc $=\operatorname{Per}^{ \pm}$или Dir, а потенииал $v \in L^{2}([0, \pi])$. Если

$$
\operatorname{Re} \lambda \geqslant N_{*}^{2}-N_{*}, \quad N_{*}=C(1+\|v\|),
$$

где $C>1$ - абсолютная постоянная, то резольвента $R(\lambda)$ вполне определена вне множества

$$
D^{N_{*}}=\bigcup_{n>N_{*}} D(n ; r), \quad \text { əде } \quad r=N_{*} / 2 .
$$

Eсли $\operatorname{Re} \lambda \leqslant N_{*}^{2}-N_{*}$, то резольвента $R(\lambda)$ вполне определена вне венецианского окна

$$
\Pi(v)=\left\{\lambda=x+i t \in \mathbb{C}: x \leqslant 0,|x|^{2}+|t|^{2} \leqslant R_{*}^{2}\right\} \cup\left\{\lambda: 0 \leqslant x \leqslant R_{*}^{3 / 2},|t| \leqslant R_{*}\right\},
$$

¿де

$$
R_{*}=C(1+\|v\|)^{4 / 3} .
$$

(Аналог этой теоремы для операторов Дирака дан в п. 1.6.)

ДоказАтельство. Мы покажем, что

$$
\left\|V R_{\lambda}^{0}\right\| \leqslant 1 / 2, \quad \text { если } \quad \lambda \notin \Pi(v) \cup D^{N_{*}}
$$

(конечно, тогда соотношение (1.21) выполнено, и резольвента $R_{\lambda}$ будет вполне определена по (1.20)). Если $\operatorname{Re} \lambda \geqslant N_{*}^{2}-N_{*}$ и $\lambda \notin D^{N_{*}}$, то $\lambda=n^{2}+x+i t$ для некоторого $n>N_{*}$ и $x^{2}+t^{2} \geqslant r^{2}$. Следовательно, в силу (1.108) и (1.102)

$$
\left\|V R_{\lambda}^{0}\right\| \leqslant C\|v\|(1 / r+1 / n) \leqslant 1 / 2
$$

по предпосылкам теоремы. 
Если $\operatorname{Re} \lambda \leqslant N_{*}^{2}-N_{*}$, то мы получаем неравенство

$$
\left\|V R_{\lambda}^{0}\right\| \leqslant 1 / 2
$$

используя (1.108) и (1.109) и соответственно двигаясь в (1.102) от третьей строчки к первой по мере того, как $\lambda=x+i t$ движется из левой полуплоскости $x \leqslant 0$ в полосы $H_{j}$ с более высокими индексами. Это приводит к специальной форме области П $(v)$. Теорема 9 доказана.

ЗАмечАниЕ 10 . Область $D=D^{N_{*}}$ не может быть построена как объединение кругов $D\left(n ; r_{n}\right)$, стягивающихся к точке, т.е. с последовательностью $r_{n} \rightarrow 0$, потому, что сдвиги потенциала $v \rightarrow v+c$ сдвигают спектр, так что линейный порядок зависимости радиусов кругов от нормы $\|v\|$ не может быть улучшен. Однако если $v_{0}=\frac{1}{\pi} \int_{0}^{\pi} v(x) d x=0$, то возможно выбрать $r_{n} \rightarrow 0$ (см. [34], [42], [29]). Наши дальнейшие теоремы тоже объясняют этот факт, но мы не будем больше подчеркивать явно это обстоятельство.

С неравенствами (1.96) и (1.94) мы имеем хорошую оценку и для нормы резольвенты $\left\|R_{\lambda}^{0} V R_{\lambda}^{0}\right\|_{2 \rightarrow \infty}$. Действительно, простое мультипликативное неравенство дает:

$$
\left\|R_{\lambda}^{0} V R_{\lambda}^{0}\right\|_{2 \rightarrow \infty} \leqslant\left\|R_{\lambda}^{0}\right\|_{2 \rightarrow \infty}\|V\|_{\infty \rightarrow 2}\left\|R_{\lambda}^{0}\right\|_{2 \rightarrow \infty},
$$

и, тем самым,

$$
\left\|R_{\lambda}^{0} V R_{\lambda}^{0}\right\|_{2 \rightarrow \infty} \leqslant\|v\| b(\lambda), \quad b \text { определено в }(1.95) .
$$

По теореме 9

$$
\left(\operatorname{Sp}\left(L_{\mathrm{bc}}\right) \cup \operatorname{Sp}\left(L_{\mathrm{bc}}^{0}\right)\right) \cap H_{n} \subset D(n ; r),
$$

так что в силу соотношений (1.41) и (1.117) мы имеем:

$$
\left\|P_{n}-P_{n}^{0}\right\|_{2 \rightarrow \infty} \leqslant \frac{\|v\|}{\pi} \int_{K} b(\lambda)|d \lambda|
$$

для любого контура $K \subset H_{n}$, содержащего круг $D(n ; r)$. Для $n \geqslant N_{*}$ выберем

$$
K=\left\{n^{2}+x+i y \in \mathbb{C}:|x|^{2}+|y|^{2}=n^{2} / 4\right\} .
$$

Тогда по лемме 7 и неравенству (1.108) мы имеем:

$$
b(\lambda) \leqslant C / n^{2}, \quad \text { если } \lambda \in K,
$$

так что в силу (1.119)

$$
\left\|P_{n}-P_{n}^{0}\right\|_{2 \rightarrow \infty} \leqslant \frac{\|v\|}{\pi} \cdot 2 \pi \cdot \frac{n}{4} \cdot \frac{C}{n^{2}}=\frac{C}{2} \cdot \frac{\|v\|}{n} .
$$

Мы доказали следующее утверждение.

ПреДЛОЖеНИЕ 11. Для операторов Хилла-Шрёдингера с граничными условиями $\mathrm{bc}=\operatorname{Per}^{ \pm}$или Dir, если $n \geqslant n_{2}(\|v\|)$, мы имеем:

$$
\left\|P_{n}-P_{n}^{0}\right\|_{2 \rightarrow \infty} \leqslant \frac{C}{n}\|v\| .
$$


Конечно, такие оценки важны для асимптотических результатов. Однако чтобы позаботиться обо всех собственных (и присоединенных) функциях, мы рассмотрим проекторы Рисса

$$
S_{n}=\frac{1}{2 \pi i} \int_{\partial \Pi_{n}(r)} R_{\lambda} d \lambda, \quad n \geqslant N_{*},
$$

где

$$
\begin{gathered}
\Pi_{n}(r)=\left\{z=x+i y \in \mathbb{C}: x \leqslant 0,|x|^{2}+|y|^{2} \leqslant r^{2}\right\} \cup\left\{z: 0 \leqslant x \leqslant n^{2}+n,|y| \leqslant r\right\}, \\
r \geqslant r_{*}=C(1+\|v\|)^{4 / 3} .
\end{gathered}
$$

По теореме 9 резольвента $R_{\lambda}$ аналитична вне определенного в (1.112) венецианского окна П, и поэтому из теоремы Коши вытекает, что $S_{n}$ не зависит от выбора $r$, если выполнено (1.125). По тем же соображениям для достаточно больших $n$

$$
S_{n}=S_{N_{*}}+\sum_{N_{*}}^{n} P_{n} .
$$

Если $v \equiv 0$, т.е. $L=L^{0}$, то мы обозначим эти проекторы через $S_{n}^{0}$. В силу $(1.41)$ для $\gamma=2, \infty$ мы имеем:

$$
\begin{aligned}
\left\|S_{n}-S_{n}^{0}\right\|_{2 \rightarrow \gamma} & \leqslant \frac{1}{2 \pi}\left\|\int_{\partial \Pi_{n}(r)}\left(R_{\lambda}-R_{\lambda}^{0}\right) d \lambda\right\| \\
& \leqslant \frac{1}{2 \pi} \int_{\partial \Pi_{n}(r)}\left\|R_{\lambda}-R_{\lambda}^{0}\right\||d \lambda|,
\end{aligned}
$$

и в силу (1.93)-(1.96)

$$
\left\|S_{n}-S_{n}^{0}\right\|_{2 \rightarrow \infty} \leqslant \frac{\|v\|}{2 \pi} \int_{\partial \Pi_{n}(r)} b(\lambda) d \lambda,
$$

или, в силу (1.93), (1.96) и (1.97),

$$
\left\|S_{n}-S_{n}^{0}\right\|_{2 \rightarrow 2} \leqslant \frac{\|v\|}{2 \pi} \int_{\partial \Pi_{n}(r)}(b(\lambda))^{1 / 2} \rho(\lambda) d \lambda \quad \forall r \geqslant r_{*}
$$

(определение $\rho(\lambda)$ см. в (1.93)). Неравенство (1.128) гарантирует, что для любого фиксированного $n$

$$
S_{n} f=S_{n}^{0} f+\left(S_{n} f-S_{n}^{0} f\right)
$$

есть непрерывная функция. Если $n=N_{*}$, пусть $B^{*}$ будет соответствующим значением правой части в (1.127). Тогда, в силу (1.130), для $\|f\|=1$ мы имеем:

$$
\left\|S_{N_{*}} f\right\|=\left\|S_{N_{*}}^{0} f\right\|+B^{*} \leqslant 2 N_{*}^{1 / 2}+B^{*},
$$

поскольку $\operatorname{dim}\left(\operatorname{Range}\left(S_{N_{*}}^{0}\right)\right) \leqslant 4 N_{*}$ и

$$
\left|\sum_{-T}^{T} a_{k} e^{i k x}\right| \leqslant(2 T+1)^{1 / 2}\left(\sum\left|a_{k}\right|^{2}\right)^{1 / 2} .
$$

Теперь мы можем утверждать следующее. 
ПРЕДЛОЖЕНИЕ 12. В условиях предложения 11 определенные в (1.126) суммы $S_{n}$ риссовских проекторов сходятся в $L^{2}$, т.е.

$$
\lim S_{n} f=f \quad \forall f \in L^{2} .
$$

Более того, в риссовских подпространствах

$$
E^{*}=\operatorname{Range}\left(S_{N_{*}}\right), \quad E_{n}=\operatorname{Range}\left(P_{n}\right), \quad n \geqslant N_{*},
$$

все функции непрерывны, и их $L^{\infty}-u L^{2}$-нормы равномерно эквивалентны, т.е. с некоторой абсолютной постоянной $C_{1}>0$ мы имеем:

$$
\|f\| \leqslant C_{1}\|f\|_{2}, \quad \text { если } f \in E^{*} \text { или } E_{n}, n \geqslant N_{*} .
$$

ДокАЗАТЕЛЬСтво. В равенствах (1.123) и (1.124) мы можем выбрать любое $r \geqslant r_{*}$, где $r_{*}$ дано в (1.125). Мы имеем:

$$
\partial \Pi(r)=\Gamma_{0}(r) \cup \Gamma^{ \pm}(r) \cup \Gamma_{1}(r),
$$

где

$$
\begin{aligned}
\Gamma_{0}(r) & =\left\{z=x+i y:|x|^{2}+|y|^{2}=r^{2}, x \leqslant 0\right\}, \\
\Gamma^{ \pm}(r) & =\left\{z=x+i r: 0 \leqslant x \leqslant n^{2}+n\right\}, \\
\Gamma_{1}(r) & =\left\{z=n^{2}+n+i t:|t| \leqslant r\right\} .
\end{aligned}
$$

Мы опускаем неравенства, которые объясняют, что интегралы

$$
\int_{\Gamma_{0}}(b(\lambda))^{1 / 2} \rho(\lambda) d \lambda \quad \text { и } \quad \int_{\Gamma^{ \pm}}(b(\lambda))^{1 / 2} \rho(\lambda) d \lambda
$$

стремятся к нулю, когда $r \rightarrow \infty$. Это вместе с (1.129) приводит нас к неравенству

$$
\left\|S_{n}-S_{n}^{0}\right\|_{2 \rightarrow 2} \leqslant\|v\| \int_{0}^{\infty} \frac{1}{n+t}(g(n, t))^{1 / 2} d t .
$$

Используя (1.102), можно показать, что правая часть этого неравенства не превосходит $C / n$. Следовательно,

$$
\left\|S_{n}-S_{n}^{0}\right\|_{2 \rightarrow 2} \rightarrow 0
$$

и соотношение $(1.132)$ выполнено.

Для $f \in \operatorname{Range}\left(P_{n}\right), n \geqslant N_{*}$, c $\|f\|_{2}=1$ мы имеем:

$$
f=P_{n} f=P^{0} f+\left(P_{n} f-P_{n}^{0} f\right),
$$

и, в силу (1.121), так как (1.130) и (1.131) выполнены, то

$$
\|f\|_{\infty} \leqslant\left\|P_{n}^{0} f\right\|_{\infty}+\left\|\left(P_{n}-P_{n}^{0}\right) f\right\|_{\infty} \leqslant 2\left\|P_{n}^{0}\right\|_{2}+\frac{C}{2 n}\|v\| \leqslant C_{1},
$$

где $C_{1}=2+C\|v\|$. Это завершает доказательство соотношения (1.134).

Напомним, наконец, что все функции в подпространствах (1.133) непрерывны, так как в силу (1.6) область определения оператора $L_{\mathrm{bc}}$ состоит из непрерывных функций. Предложение 12 доказано. 
Аналоги предложений 11 и 12 в случае Дирака немного сложнее. Важное отличие состоит в том, что такая оценка не может быть дана с последовательностью $\delta_{n} \rightarrow 0$ (вместо $C\|v\| / n$ ), зависящей только от $L^{2}$-нормы потенциала $v$. Она будет зависеть от индивидуального $V \in\left(L^{2}(I)\right)^{2}$ или от компактного множества потенциалов. См. детали в п. 1.6.

\section{6. Оценки резольвентных норм и локализация спектра оператора} Дирака; уклонение собственных подпространств свободного и возмущенного операторов. Мы дадим теперь аналог построений п. 1.5 в случае операторов Дирака. Напомним, что $R_{\lambda}^{0}-$ это диагональный оператор с матричным представлением $(1 /(\lambda-k))_{k \in \Gamma_{\mathrm{bc}}}$ (см. п. 1.1) и

$$
\begin{gathered}
\left\|R_{\lambda}^{0}\right\|=\max _{k \in \Gamma_{\mathrm{bc}}} \frac{1}{|\lambda-k|} ; \\
\left\|R_{\lambda}^{0}\right\|_{2 \rightarrow \infty} \leqslant\left\|r_{\lambda}^{0}: \ell^{2} \rightarrow \ell^{1}\right\| \leqslant(a(\lambda))^{1 / 2},
\end{gathered}
$$

где

$$
a(\lambda)=\sum_{k \in \Gamma_{\mathrm{bc}}} \frac{1}{|\lambda-k|^{2}} .
$$

Ho

$$
\begin{array}{lll}
a(\lambda)=a(\lambda+2), & \text { если } & \mathrm{bc}=\mathrm{Per}^{ \pm}, \\
a(\lambda)=a(\lambda+1), & \text { если } & \mathrm{bc}=\mathrm{Dir},
\end{array}
$$

не будучи малым при вещественных $\lambda$ между $n$ и $n+1$. Поэтому мы не можем надеяться получить аналог леммы 7, т.е. оценок вроде (1.108) и (1.109) с некоторой последовательностью $\delta_{n} \rightarrow 0$ в правой части.

ЛЕмма 13. Пусть

$$
\lambda=n+x+i t, \quad-1 \leqslant x \leqslant 1 .
$$

Тогда

$$
a(\lambda) \leqslant \sum_{\tau=-1}^{+1} \frac{1}{(x-\tau)^{2}+t^{2}}+6 \min \left(1, \frac{1}{|t|}\right) .
$$

ДокАЗАТЕЛЬСтво. В силу (1.139) и (1.140) без потери общности можно рассмотреть только тот случай, когда $n=0$ или $n=1$. Если забрать из (1.138) три слагаемых с $k=0, \pm 1$ для bc $=$ Dir, или два члена с $k= \pm 1$, если bc $=\mathrm{Per}^{-}$, или одно слагаемое с $k=0$, если $\mathrm{bc}=\mathrm{Per}^{+}$, то мы можем оценить их сверху первым членом в правой части (1.142). Остающаяся сумма не превосходит

$$
2 \sum_{k=1}^{\infty} \frac{1}{k^{2}+t^{2}} \leqslant 2\left(\frac{1}{1+t^{2}}+\int_{1}^{\infty} \frac{1}{x^{2}+t^{2}} d x\right) \leqslant 6 \min \left(1, \frac{1}{|t|}\right) .
$$

Это завершает доказательство.

В силу (1.137) лемма 13 дает следующие оценки:

$$
\left\|R_{\lambda}^{0}\right\|_{2 \rightarrow \infty}^{2} \leqslant \frac{3}{t^{2}}+6 \min \left(1, \frac{1}{|t|}\right), \quad t \neq 0
$$


или $\left\|R_{\lambda}^{0}\right\| \leqslant 3 / \sqrt{t}$, если $|t| \geqslant 1$; если, кроме того, $x=n+1 / 2$ полуцело, то

$$
\left\|R_{\lambda}^{0}\right\|^{2} \leqslant \frac{12}{1+4 t^{2}}+6 \min \left(1, \frac{1}{|t|}\right)
$$

так что

$$
\left\|R_{\lambda}^{0}\right\|^{2} \leqslant 12 \min \left(1, \frac{1}{|t|}\right) .
$$

В силу (1.143) мы имеем:

$$
\left\|V R_{\lambda}^{0}\right\| \leqslant\left\|R_{\lambda}^{0}\right\|_{2 \rightarrow \infty}\|V\|_{\infty \rightarrow 2} \leqslant 1 / 2, \quad \text { если }|t| \geqslant(1+14\|v\|)^{2} .
$$

Таким образом, в силу (1.20) выполнено следующее.

Лемма 14. Резольвента $R_{\lambda}$ вполне определена как аналитическая операторнозначная функиия на объединении двух полуплоскостей

$$
\left\{\lambda:|\operatorname{Im} \lambda| \geqslant(1+14\|v\|)^{2}\right\}
$$

Более того,

$$
\left\|R_{\lambda}\right\|_{2 \rightarrow 2} \leqslant 2\left\|R_{\lambda}^{0}\right\|_{2 \rightarrow 2}=\frac{2}{\operatorname{dist}\left(\lambda, \operatorname{Sp}\left(L_{\mathrm{bc}}^{0}\right)\right)}=\frac{2}{\sqrt{(x-n)^{2}+t^{2}}},
$$

где $n \in \operatorname{Sp}\left(L_{\mathrm{bc}}^{0}\right)$ - ближайшая $\kappa \lambda$ точка, $u$

$$
\left\|R_{\lambda}\right\|_{2 \rightarrow \infty}+\left\|R_{\lambda}\right\|_{1 \rightarrow 2} \leqslant 2\left\|R_{\lambda}^{0}\right\|_{2 \rightarrow \infty}+2\left\|R_{\lambda}^{0}\right\|_{1 \rightarrow 2} \leqslant 4(a(\lambda))^{1 / 2} .
$$

Таким образом, теперь мы можем использовать соотношение (1.142).

Но, как было замечено после (1.139)-(1.140), это не может нам помочь получить хорошие оценки и построить резольвенту $R_{\lambda}, \lambda=n+x+i t$, при малых $|t|$ и $x$, отличных от нуля, даже если $|n|$ велико. Однако ряд Неймана

$$
(1-T)^{-1}=1+T+T^{2}+\cdots
$$

сходится (даже если неравенство $\|T\|<1$ не выполнено), если $\|T\|^{m}<1$ для некоторого $m>1$. В этом случае

$$
\sum_{k=0}^{\infty} T^{k}=\sum_{j=0}^{m-1} T^{j}\left(\sum_{i=0}^{\infty}\left(T^{m}\right)^{i}\right)=\left(1+T+T^{2}+\cdots+T^{m-1}\right)\left(1-T^{m}\right)^{-1}
$$

Мы используем только случай $m=2$. Тогда если $T=V R_{\lambda}^{0}$ или $T=R_{\lambda}^{0} V$, то мы имеем:

$$
\left(V R_{\lambda}^{0}\right)^{2}=V\left(R_{\lambda}^{0} V R_{\lambda}^{0}\right)
$$

и

$$
\left(R_{\lambda}^{0} V\right)^{2}=\left(R_{\lambda}^{0} V R_{\lambda}^{0}\right) V
$$

Следовательно,

$$
\left\|\left(V R_{\lambda}^{0}\right)^{2}\right\| \leqslant\|v\|\left\|R_{\lambda}^{0} V R_{\lambda}^{0}\right\|_{2 \rightarrow \infty}
$$

и

$$
\left\|\left(R_{\lambda}^{0} V\right)^{2}\right\| \leqslant\|v\|\left\|R_{\lambda}^{0} V R_{\lambda}^{0}\right\|_{1 \rightarrow 2}
$$

как упомянуто в (1.35) и (1.36). Оценки норм в правой части (1.150) и (1.151) получаются точно так же. 
ПреДЛОЖЕНИЕ 15. В случае Дирака рассмотрим

$$
\lambda=n+x+i t, \quad \text { где }|x| \leqslant 1 \text { для } \mathrm{bc}=\mathrm{Per}^{ \pm}, \quad|x| \leqslant 1 / 2 \text { для bc=Dir }
$$

$u n \in 2 \mathbb{Z} \partial л я \mathrm{bc}=\mathrm{Per}^{+}, n \in 1+2 \mathbb{Z} \partial л я \mathrm{bc}=\mathrm{Per}^{-}, n \in \mathbb{Z} \partial л я \mathrm{bc}=$ Dir. Тогда для достаточно больиих $|n|$

$$
\left\|R_{\lambda}^{0} V R_{\lambda}^{0}\right\|_{2 \rightarrow \infty} \leqslant C \delta(n ; r)
$$

где $C$ - абсолютная постоянная, $r=\sqrt{x^{2}+t^{2}} u$

$$
\delta(n ; r)= \begin{cases}\frac{\|V\|}{\sqrt{|n|}}+\mathscr{E}_{|n| / 2}(w), & r \geqslant \frac{1}{8}, \\ \frac{|w(2 n)|}{r^{2}}+\frac{\|V\|}{\sqrt{|n|}}+\mathscr{E}_{|n| / 2}(w), & r<\frac{1}{8},\end{cases}
$$

a $w \in \ell^{2}$ определено леммой 2 .

ДокАЗАтЕЛЬство. По лемме 2 матричное представление $R_{\lambda}^{0} V R_{\lambda}^{0}$ задается в (1.76) для $a$. В силу (1.72) его норма не превосходит суммы $(\Sigma(n))^{1 / 2}$, приходящей из правой части в (1.78) (лемма 4). Упрощение правой части в (1.78) дает нам последовательность $\delta(n, r)$ для (1.154) и неравенства (1.153). Предложение 15 доказано.

СлЕДСтвиЕ 16. В обозначениях предложения 15 если $K$ - компактное множество потенииалов $v \in\left(L^{2}(I)\right)^{2}$, то существует такая последовательность $\delta^{*}(n) \rightarrow 0$, что для всех $v \in K$

$$
\left\|R_{\lambda}^{0} V R_{\lambda}^{0}\right\|_{2 \rightarrow \infty} \leqslant \delta^{*}(|n|), \quad \text { ecлu } \quad r \geqslant \frac{1}{8} .
$$

ДокАЗАтельство. По лемме $2 w=H(v)$, где $H$ - ограниченный оператор, так что $H(K)$ - тоже компактное множество. Поэтому $\|v\| \leqslant M$ для $v \in K$, и

$$
\varepsilon_{n}:=\sup \left\{\mathscr{E}_{|n| / 2}(w): w \in H(K)\right\} \rightarrow 0 .
$$

Но тогда в силу (1.153) и (1.154) последовательность $\delta^{*}(n)=C\left(M / \sqrt{n}+\varepsilon_{n}\right)$ может быть выбрана, с тем чтобы (1.155) было выполнено для всех $V \in K$. Следствие 16 доказано.

Мы дадим теперь аналог теоремы 9.

ТЕорема 17 (локализационная теорема для операторов Дирака). Пусть $L_{\mathrm{bc}}$ определен соотношением (1.9) с граничными условиями $\mathrm{bc}=\mathrm{Per}^{ \pm}$или Dir u потенциалом $v \in\left(L^{2}(I)\right)^{2}$ как в (1.17). Тогда существует такое $N_{*}=N_{*}(v)$, что при всех $|\operatorname{Re} \lambda| \geqslant N_{*}$ резольвента $R_{\lambda}=\left(\lambda-L_{\mathrm{bc}}\right)^{-1}$ вполне определена вне множества

$$
D^{N_{*}}=\bigcup_{|n|>N_{*}} D(n ; 1 / 8) .
$$

Eсли $|\operatorname{Re} \lambda| \leqslant N_{*}$, то $R(\lambda)$ вполне определена вне прямоугольника

$$
\Pi(T)=\left\{\lambda=x+i t \in \mathbb{C}:|x| \leqslant N_{*},|t| \leqslant T=10(1+\|v\|)\right\} .
$$


ДокАЗАТЕЛьство. По лемме 14 резольвента $R_{\lambda}$ вполне определена вне горизонтальной полосы $\{\lambda:|\operatorname{Im} \lambda| \leqslant T\}$. Это доказывает вторую часть теоремы.

По (1.154) мы выберем $N_{*}$, чтобы гарантировать, что

$$
\|v\| \cdot C \delta(n ; 1 / 8) \leqslant 1 / 2, \quad \text { если }|n| \geqslant N_{*} .
$$

Тогда $\left\|V R_{\lambda} V R_{\lambda}\right\| \leqslant 1 / 2$ для $\lambda$ как в $(1.152), r \geqslant 1 / 8$ и резольвента

$$
R_{\lambda}=R_{\lambda}^{0}\left(1+V R_{\lambda}^{0}\right)\left(1-V R_{\lambda}^{0} V R_{\lambda}^{0}\right)^{-1}
$$

вполне определена, если $\lambda \notin D^{N_{*}}$ и $|\operatorname{Re} \lambda| \geqslant N_{*}$. Это завершает доказательство теоремы 17.

Естественна следующая модификация (и уточнение) этого утверждения.

Теорема 18. В условиях и при обозначениях теоремы 17 оператор Дирака $L_{\mathrm{bc}}$ с граничными условиями $\mathrm{bc}=\mathrm{Per}^{ \pm}$или Dir имеет дискретный спектр $\operatorname{Sp}\left(L_{\mathrm{bc}}\right)$. Размерности его проекторов Коши-Рисса те же самые, что и в случае свободного оператора $L_{\mathrm{bc}}^{0}$. Точнее, для $|k|>N_{*}=2 m+1 / 2$

$$
\operatorname{dim} P_{k}(b c)=\operatorname{dim} P_{k}^{0}(b c)= \begin{cases}2 & \text { для четных } k, \mathrm{bc}=\mathrm{Per}^{+}, \\ 0 & \text { для нечетных } k, \mathrm{bc}=\mathrm{Per}^{+}, \\ 0 & \text { для четных } k, \mathrm{bc}=\mathrm{Per}^{-}, \\ 2 & \text { для нечетных } k, \mathrm{bc}=\mathrm{Per}^{-}, \\ 1 & \text { для четных } k, \mathrm{bc}=\mathrm{Dir}, \\ 1 & \text { для нечетных } k, \mathrm{bc}=\mathrm{Dir} .\end{cases}
$$

Eсли

$$
P_{*}=\frac{1}{2 \pi i} \int_{\partial \Pi(T)}\left(z-L_{\mathrm{bc}}\right)^{-1} d z
$$

mo

$$
\operatorname{dim} P_{*}=\operatorname{dim} P_{*}^{0}= \begin{cases}2(2 m+1), & \mathrm{bc}=\text { Per }^{+}, \\ 2 \cdot 2 m, & \mathrm{bc}=\text { Per }^{-}, \\ 4 m+1, & \mathrm{bc}=\text { Dir } .\end{cases}
$$

ДокАзАтЕЛьство. Теорема 17 утверждает, что

$$
\sigma\left(L_{\mathrm{bc}}(v)\right) \subset \Pi(T) \cup D_{*}^{N} .
$$

Если $N_{*}$ достаточно велико, то резольвента $R_{\lambda}(V)$ есть аналитическая операторнозначная функция вне множества $G=\Pi(T) \cup D^{N_{*}}$ и на его границе $\partial G$. Если мы рассмотрим семейство потенциалов $v_{\zeta}=\zeta v,|\zeta| \leqslant 1$, то все они удовлетворяют условиям (1.153) и (1.154), где $v, w$ заменены на $\zeta v, \zeta w$, и резольвента

$$
R_{\lambda}(\zeta v)=R_{\lambda}^{0}\left(1+\zeta V R_{\lambda}^{0}\right)\left(1-\zeta^{2} V R_{\lambda}^{0} V R_{\lambda}^{0}\right)^{-1}
$$

вполне определена и аналитична по $\lambda$ в замыкании $\mathbb{C} \backslash G$ и по $\zeta$, если $|\zeta| \leqslant 1$. Но мы знаем все о свободном операторе $L_{\mathrm{bc}}^{0}$ и его спектре $\operatorname{Sp}\left(L_{\mathrm{bc}}^{0}\right)-$ см. п. 1.1 . Для $|\zeta| \leqslant 1$ функция

$$
P_{k}(\zeta)=\frac{1}{2 \pi i} \int_{\partial D(k ; 1 / 8)}\left(z-L_{\mathrm{bc}}(\zeta V)\right)^{-1} d z
$$


непрерывна, так что

$$
\operatorname{dim} P_{k}(\zeta)=\operatorname{Trace} P_{k}(\zeta)
$$

постоянна как целочисленнозначная функция. То же самое верно для

$$
P_{*}(\zeta)=\frac{1}{2 \pi i} \int_{\partial \Pi}\left(z-L_{\mathrm{bc}}(\zeta V)\right)^{-1} d z .
$$

Формулы (1.160), (1.162) вытекают из информации п. 1.1 о свободном операторе $L_{\mathrm{bc}}^{0}$ и его собственных подпространствах $E_{\mathrm{bc}}^{0}$ в случае Дирака. Теорема 18 доказана.

ПРЕДЛОЖЕНИЕ 19. Для операторов Дирака с граничными условиями bc = $\mathrm{Per}^{ \pm}$или Dir если $|n|$ достаточно велико, то

$$
\left\|P_{n}^{\mathrm{bc}}-P_{n}^{0}: L^{2} \rightarrow L^{\infty}\right\|=: \kappa_{n} \rightarrow 0
$$

¿əe

$$
\kappa_{n} \leqslant \kappa_{n}^{*}:=C\|v\|\left(\frac{\|v\|}{\sqrt{|n|}}+\mathscr{E}_{|n| / 2}(w)\right) .
$$

ДокАЗАТЕЛЬство. В силу неравенства (1.142) из леммы 13 , где $\lambda=n+x+i t$, и равенства $T=V R_{\lambda}^{0}$ мы имеем

$$
\|T\| \leqslant 4\left(\frac{1}{r}+\frac{1}{\sqrt{1+|t|}}\right)\|v\|, \quad r=\sqrt{x^{2}+t^{2}},
$$

а по предложению 15

$$
\left\|T^{2}\right\| \leqslant C\|v\|\left(\frac{\|v\|}{\sqrt{|n|}}+\mathscr{E}_{|n| / 2}(w)\right), \quad r \leqslant \frac{1}{8} .
$$

Следовательно,

$$
P_{n}^{\mathrm{bc}}-P_{n}^{0}=\frac{1}{2 \pi i} \int_{|z-n|=1 / 4}\left(R_{z}-R_{z}^{0}\right) d z
$$

и в силу (1.28) мы имеем, что

$$
\begin{aligned}
\left\|P_{n}^{\mathrm{bc}}-P_{n}^{0}: L^{2} \rightarrow L^{\infty}\right\| & \leqslant \frac{1}{2 \pi} \int_{|z-n|=1 / 4}\left\|R_{z}^{0} V R_{z}^{0}: L^{2} \rightarrow L^{\infty}\right\|(1+\|T\|) 2|d z| \\
& \leqslant \frac{16}{2 \pi} \cdot 2 \pi \cdot \frac{1}{4} \kappa_{n}^{*} \rightarrow 0
\end{aligned}
$$

где $\kappa_{n}^{*}$ дано в (1.165). Предложение 19 доказано.

Следующее предложение аналогично предложению 12.

ПРЕДЛОЖЕНИЕ 20. В предпосылках и обозначениях предложения 19 частичные суммы проекторов Рисса

$$
S_{n}=\frac{1}{2 \pi i} \int_{\partial \Pi_{n}(r)} R_{\lambda} d \lambda, \quad n \geqslant N^{*},
$$

где

$$
\Pi_{n}(r)=\{z=x+i y \in \mathbb{C}:|x| \leqslant n+1 / 2,|y| \leqslant r\}, \quad r \geqslant r_{*},
$$


сходятся в $L^{2} \kappa 1$, m.e.

$$
\lim S_{n} F=F \quad \forall F \in\left(L^{2}(I)\right)^{2} .
$$

Более того, все функции в риссовских подпространствах

$$
E^{*}=\operatorname{Range}\left(S_{N_{*}}\right), \quad E_{n}=\operatorname{Range}\left(P_{n}\right), \quad n \geqslant N_{*},
$$

непрерывны и их $L^{2}-u L^{\infty}$-нормы равномерно эквивалентны, т.е. с некоторой абсолютной постоянной $C_{1}>0$

$$
\|F\|_{\infty} \leqslant C_{1}\|F\|_{2}, \quad F \in E^{*} \text { или } E_{n}, \quad n \geqslant N_{*} .
$$

Мы опускаем детали доказательства, поскольку оно проводится по той же схеме, что была использована в доказательстве предложения 12, но теперь решающую роль играют неравенства (1.150), (1.153) и (1.136)-(1.138), (1.142).

1.7. Примечания. Важные шаги в спектральной теории периодических операторов были сделаны И. М. Гельфандом [43] и (особенно в несамосопряженном случае) Ф. С. Рофе-Бекетовым [44], [45].

Общая схема теории возмущений хорошо известна и рутинно используется в спектральном анализе. В этом отношении мы пишем хорошо известные факты и формулы, вводя полезные для дальнейшего обозначения и элементарные неравенства.

В случае Хилла-Шрёдингера оценки резольвентных норм и утверждения о локализации спектра можно найти в [42], [34] и других классических источниках, хотя часто изложение ограничивается вещественными потенциалами, тогда как интерес к структуре и асимптотике собственных функций требует дополнительных рассмотрений или более сложной техники.

В случае Дирака основные результаты (теоремы 17, 18 и предложения 19, 20) приходят из [46], [47], хотя наше изложение дает существенные технические упрощения. Последнее отчасти связано с матричным представлением операторов умножения и их ганкелевой структурой (леммы 1,2 ), что любопытно даже в случае Хилла-Шрёдингера с граничными условиями Дирихле. Это отличает наш подход от других реализаций оператора умножения (ср., например, [48], [49]).

\section{2. Основное уравнение; оценки для $\left|\gamma_{n}\right|$ сверху}

2.1. Редукционная схема. Наш подход к анализу спектральных лакун основан на теории возмущений (см. [40], [41]). В этом пункте мы даем общую редукционную схему в той форме, которая наилучшим образом соответствует нашим целям.

Пусть $H$ - гильбертово пространство, а $L^{0}$ и $V$ - (неограниченные) операторы, действующие в $H$. Предположим, что оператор $L^{0}$ самосопряженный и выполнены следующие условия:

(i) $E^{0}$ есть инвариантное подпространство оператора $L^{0}$;

(ii) $P^{0}$ - ортогональный проектор на $E^{0}$ и $Q^{0}=1-P^{0}$; 
(iii) $U$ есть множество таких комплексных чисел, что

$$
\operatorname{Sp}\left(P^{0} L^{0} P^{0}\right) \subset U, \quad U \cap \operatorname{Sp}\left(Q^{0} L^{0} Q^{0}\right)=\varnothing,
$$

где $\operatorname{Sp}(\cdot)$ обозначает спектр соответствующего оператора.

В силу (2.1) для каждого $\lambda \in U$ ограничение оператора $\left(\lambda-L^{0}\right)$ на область значений проектора $Q^{0}$ имеет обратный оператор $D: Q^{0}(H) \rightarrow Q^{0}(H)$. Положим

$$
T_{\lambda}=V D Q^{0}: H \rightarrow H .
$$

Конечно, операторы $D$ и $Q^{0}$ зависят от $\lambda$, но эта зависимость не показана в обозначениях.

Лемма 21. Предположим, что для каждого $\lambda \in U$ оператор $T=T_{\lambda}$ ограничен и обратный $(1-T)^{-1}$ существует. Тогда для $\lambda \in U$

(а) $\lambda \in \operatorname{Sp}(L)$ тогда и только тогда, когда $\lambda \in \operatorname{Sp}\left(P^{0} L^{0} P^{0}+S\right)$, где

$$
S=S(\lambda)=P^{0}(1-T)^{-1} V P^{0}: E^{0} \rightarrow E^{0}
$$

(b) $\lambda$ есть собственное значение оператора $L$ тогда и только тогда, когда $\lambda$ есть собственное значение оператора $P^{0} L^{0} P^{0}+S$.

Более того,

$$
\begin{gathered}
L f=\lambda f, \quad f \neq 0 \quad \Rightarrow \quad\left(L^{0}+S\right) P^{0} f=\lambda P^{0} f, \quad P^{0} f \neq 0, \\
\left(L^{0}+S\right) f_{1}=\lambda f_{1}, \quad f_{1} \in E^{0} \quad \Rightarrow \quad L f=\lambda f, \quad f=f_{1}+D Q^{0}(1-T)^{-1} f_{1} .
\end{gathered}
$$

Доказательство. Пусть $\lambda \in U \backslash \mathrm{Sp}(L)$; тогда для каждого $g \in H$ существует единственный вектор $f \in H$ такой, что

$$
\left(\lambda-L^{0}-V\right) f=g
$$

и $f$ зависит линейно и непрерывно от $g$. Это уравнение эквивалентно системе

$$
\begin{aligned}
& P^{0}\left(\lambda-L^{0}-V\right)\left(f_{1}+f_{2}\right)=g_{1}, \\
& Q^{0}\left(\lambda-L^{0}-V\right)\left(f_{1}+f_{2}\right)=g_{2},
\end{aligned}
$$

где $f_{1}=P^{0} f, f_{2}=Q^{0} f, g_{1}=P^{0} g, g_{2}=Q^{0} g$. Так как оператор $L^{0}$ самосопряженный, то область значений $Q^{0}(H)$ проектора $Q^{0}$ является инвариантным подпространством. Следовательно,

$$
P^{0} Q^{0}=Q^{0} P^{0}=0, \quad P^{0} L^{0} Q^{0}=Q^{0} L^{0} P^{0}=0,
$$

так что уравнения (2.7) и (2.8) могут быть переписаны как

$$
\begin{aligned}
& \left(\lambda-L^{0}\right) f_{1}-P^{0} V f_{1}-P^{0} V f_{2}=g_{1}, \\
& \left(\lambda-L^{0}\right) f_{2}-Q^{0} V f_{1}-Q^{0} V f_{2}=g_{2} .
\end{aligned}
$$

Действуя на обе части (2.10) оператором $V D$, мы получаем ввиду (2.2), что

$$
V f_{2}-T V f_{1}-T V f_{2}=T g_{2}
$$


Так как обратный $(1-T)^{-1}$ существует, то можно решить это уравнение относительно $V f_{2}$ :

$$
V f_{2}=(1-T)^{-1} T V f_{1}+(1-T)^{-1} T g_{2} .
$$

Теперь подставим полученное выражение для $V f_{2}$ в $(2.9)$; это дает нам соотношение

$$
\left(\lambda-L^{0}\right) f_{1}-P^{0} V f_{1}-P^{0}(1-T)^{-1} T V f_{1}=P^{0}(1-T)^{-1} T g_{2}+g_{1} .
$$

Поскольку $1+T(1-T)^{-1}=(1-T)^{-1}$, это приводит нас к соотношению

$$
\left[\lambda-L^{0}-P^{0}(1-T)^{-1} V\right] f_{1}=P^{0}(1-T)^{-1} T g_{2}+g_{1}
$$

Если $g_{2}=0$ (т.е. если $g=g_{1} \in E^{0}$ ), то (2.13) становится уравнением

$$
\left(\lambda-L^{0}-S\right) f_{1}=g_{1} \quad \text { c } \quad S=P^{0}(1-T)^{-1} V P^{0} .
$$

Ввиду (2.6) для каждого вектора $g_{1} \in E^{0}$ мы имеем: $f_{1}=P^{0}(\lambda-L)^{-1} g_{1}$, т.е. оператор $P^{0}(\lambda-L)^{-1}$ является обратным к оператору $\lambda-\left(P^{0} L^{0} P^{0}+S\right)$. Следовательно, $\lambda \notin \operatorname{Sp}\left(P^{0} L^{0} P^{0}+S\right)$.

Обратно, пусть $\lambda \in U \backslash \operatorname{Sp}\left(P^{0} L^{0} P^{0}+S\right)$. Зафиксируем вектор $g=g_{1}+g_{2} \in H$ и попытаемся решить уравнение (2.6) для $f=f_{1}+f_{2}$, или, что эквивалентно, попытаемся решить систему $(2.7),(2.8)$ для $f_{1}, f_{2}$. Из системы $(2.7),(2.8)$ вытекает уравнение (2.13). Так как $\lambda \notin \operatorname{Sp}\left(P^{0} L^{0} P^{0}+S\right)$, уравнение $(2.13)$ определяет $f_{1}$ однозначно; более того, $f_{1}$ зависит от $g$ линейно и непрерывно. Если $g=0$, то в силу (2.13) $f_{1}=0$, и поэтому, ввиду (2.12), мы имеем $V f_{2}=0$. Таким образом, из (2.8) вытекает, что $f_{2}=D Q^{0}(\lambda-T)^{-1} g_{2}=0$, так что система $(2.7),(2.8)$ имеет не более одного решения.

Положим

$$
f_{2}=D Q^{0}(1-T)^{-1} V f_{1}+D Q^{0}(1-T)^{-1} g_{2}
$$

очевидно, $f_{2}$ зависит от $g$ линейно и непрерывно. Теперь мы проверим, что пара $\left(f_{1}, f_{2}\right)$ удовлетворяет системе $(2.7),(2.8)$. Действительно, так как $T=V D Q^{0}$, то легко видеть, что уравнение (2.12) выполняется, и вектор $f_{1}$ выбран так, что (2.13) тоже выполнено. Следовательно, в силу (2.12) и (2.13) мы имеем:

$$
\begin{aligned}
\left(\lambda-L^{0}\right) f_{1} & =P^{0}(1-T)^{-1} V f_{1}+P^{0}(1-T)^{-1} T g_{2}+g_{1} \\
& =P^{0} V f_{1}+P^{0}(1-T)^{-1} T V f_{1}+P^{0}(1-T)^{-1} T g_{2}+g_{1} \\
& =P^{0} V f_{1}+P^{0} V f_{2}+g_{1}
\end{aligned}
$$

т.е. (2.7) выполнено.

Наконец, так как $\left(\lambda-L^{0}\right) D=Q^{0}$ и $(1-T)^{-1}=(1-T)^{-1} T+1$, мы получаем:

$$
\begin{aligned}
\left(\lambda-L^{0}\right) f_{2} & =Q^{0}(1-T)^{-1} V f_{1}+Q^{0}(1-T)^{-1} g_{2} \\
& =Q^{0} V f_{1}+Q^{0}(1-T)^{-1} T V f_{1}+Q^{0}(1-T)^{-1} T g_{2}+g_{2} \\
& =Q^{0} V f_{1}+Q^{0} V f_{2}+g_{2} .
\end{aligned}
$$

Таким образом, (2.8) тоже выполнено. Это заканчивает доказательство части (а). 
Чтобы доказать (b), мы используем те же самые формулы, но с вектором $g=0$. Предположим, что $\lambda$ есть собственное значение оператора $L$ и $(\lambda-L) f=$ 0 для некоторого вектора $f \neq 0$. Тогда (2.6) выполняется с вектором $g=0$, так что уравнения (2.7)-(2.13) тоже выполнены. Таким образом, в силу (2.13) мы имеем, что

$$
\left(\lambda-P^{0} L^{0} P^{0}-S\right) f_{1}=0 \quad \text { c } \quad f_{1}=P^{0} f,
$$

где $f_{1} \neq 0$. Действительно, если $f_{1}=0$, то из $(2.12)$ вытекало бы, что $V f_{2}=0$, но тогда в силу (2.10) мы имели бы $\left(\lambda-L^{0}\right) f_{2}=0$. Это влекло бы $f_{2}=0$, и мы имели бы противоречие: $f=f_{1}+f_{2}=0$.

Предположим теперь, что $\lambda$ есть собственное значение оператора $P^{0} L^{0} P^{0}+S$ и

$$
\left(\lambda-P^{0} L^{0} P^{0}-S\right) f_{1}=0, \quad f_{1} \neq 0 .
$$

Положим

$$
f_{2}=D Q^{0}(1-T)^{-1} V f_{1}, \quad f=f_{1}+f_{2} .
$$

Тогда (2.13) и (2.15) выполнены, если $g=0$, и тот же самый довод, что был использован в части (а), показывает, что $(\lambda-L) f=0$, и тем самым $\lambda \in \operatorname{Sp}(L)$. Это завершает доказательство части (b), а с ней и всей леммы 21.

Теперь мы применяем лемму 21 к операторам Хилла-Шрёдингера (или Дирака), рассматриваемым с периодическими или антипериодическими граничными условиями (см. пп. 1.1, 1.2). Тогда свободный оператор $L^{0}$ имеет дискретный спектр, скажем, $\operatorname{Sp}\left(L^{0}\right)=\left\{\lambda_{n}^{0}, n \in \Gamma_{\mathrm{bc}}\right\}$, где в случае Хилла-Шрёдингера

$$
\lambda_{n}^{0}=n^{2}, \quad \Gamma_{\text {per }}=\{0,2,4, \ldots\}, \quad \Gamma_{\text {ap }}=\{1,2,3, \ldots\},
$$

а в случае Дирака

$$
\lambda_{n}^{0}=n, \quad \Gamma_{\text {per }}=2 \mathbb{Z}, \quad \Gamma_{\text {ap }}=1+2 \mathbb{Z} .
$$

За одним исключением $\left(\lambda_{0}^{0}=0\right.$ в случае Хилла-Шрёдингера) каждое собственное значение $\lambda_{n}^{0}$ имеет геометрическую кратность 2 , так что соответствующее собственное подпространство $E^{0}=E_{n}^{0}$ двумерно. Более того, мы покажем (см. пп. $2.2,2.4)$, что для достаточно больших $|n|$ предположения леммы 21 выполнены, если $U$ выбрано как круг с центром $\lambda_{n}^{0}$ и подходящим радиусом $r=r(V)$. Когда $E^{0}$ двумерно, часть (b) леммы 21 влечет следующее.

ЗАмЕчАнИЕ 22. В предположениях и обозначениях леммы 21 если $\operatorname{dim} E^{0}=2$, то $L$ имеет собственное значение $\lambda \in U$ геометрической кратности 2 тогда и только тогда, когда $\lambda \in U$ является собственным значением оператора $P^{0} L^{0} P^{0}+S(\lambda)$ с геометрической кратностью 2 .

\section{2. Основное уравнение: случай операторов Хилла-Шрёдингера.}

Теперь мы применяем редукционную схемы из предыдущего пункта. В силу локализационной теоремы 9 , если $n>n_{0}(\|v\|)=6\|v\|$, то оператор $L^{0}+V$ имеет в точности два (подсчитанных с учетом их алгебраической кратности) собственных значения (периодических, если $n$ четно, и антипериодических, если $n$ нечетно) в круге $U=D\left(n^{2}, r\right)$ с центром $n^{2}$ и радиусом $r=n / 2$. 
Зафиксируем $n>n_{0}(\|v\|)$; пусть $E_{n}^{0}=\operatorname{Span}\left\{e_{n}^{1}=e^{-i n x}, e_{n}^{2}=e^{i n x}\right\}$ обозначает собственное подпространство оператора $L^{0}$, соответствующее собственному значению $n^{2}$. Обозначим через $P_{n}^{0}$ ортогональный проектор на $E_{n}^{0}$ и положим $Q_{n}^{0}=1-P_{n}^{0}$. Для $\lambda \in U$ ограничение оператора $\lambda-L^{0}$ на область значений проектора $Q_{n}^{0}$ имеет правый обратный оператор. Действительно, рассмотрим диагональный оператор $D=D_{n}$, заданный соотношением

$$
D e_{j}=\left[\left(\lambda-j^{2}\right)\right]^{-1} e_{j} \quad \text { для } \quad j \neq \pm n .
$$

Тогда $D$ является правым обратным к ограничению оператора $\lambda-L^{0}$ на образ проектора $Q^{0}$, т.е.

$$
\left(\lambda-L^{0}\right) D x=x \quad \text { для } \quad x \in Q_{n}^{0}(H) .
$$

Пусть $\lambda=n^{2}+z$; тогда матричное представление для $D_{n}$ задается соотношением

$$
\left(D_{n}\right)_{k m}=\frac{1}{n^{2}-k^{2}+z} \delta_{k m}, \quad k, m \in(n+2 \mathbb{Z}) \backslash\{ \pm n\},
$$

где $\delta_{k m}=0$, если $k \neq m$, и $\delta_{k m}=1$, если $k=m$. Положим, как и в $(2.2)$,

$$
T=T(n, \lambda)=V D_{n} Q_{n}^{0}
$$

Так как матричное представление $V$ (оператора умножения на функцию $\left.v(x)=\sum V(k) \exp (i k x)\right)$ задается как

$$
V_{k m}=V(m-k)
$$

то матричное представление оператора $T_{n}$ определяется соотношением

$$
\left(T_{n}\right)_{k m}=\frac{V(m-k)}{n^{2}-k^{2}+z}, \quad k, m \in(n+2 \mathbb{Z}) \backslash\{ \pm n\} .
$$

Лемма 23. Если $\lambda \in U=\left\{n^{2}+z:|z| \leqslant n / 2\right\}$, то для нормъ ГилъбертаШмидта оператора $T_{n}$ выполнено следующее неравенство:

$$
\left\|T_{n}\right\|_{\mathrm{HS}} \leqslant \frac{3}{n}\|v\|
$$

ДокАЗАТЕЛЬСтво. В силу элементарного неравенства

$$
\frac{1}{\left|n^{2}+z-j^{2}\right|} \leqslant \frac{2}{\left|n^{2}-j^{2}\right|}, \quad j \in n+2 \mathbb{Z}, \quad j \neq \pm n, \quad|z| \leqslant n / 2,
$$

и леммы 78 (см. дополнение) мы имеем:

$$
\left\|T_{n}\right\|_{\mathrm{HS}}^{2} \leqslant \sum_{j, k \neq \pm n} \frac{4|V(j-k)|^{2}}{\left|n^{2}-k^{2}\right|^{2}} \leqslant \frac{8}{n^{2}}\|v\|^{2} .
$$

Это доказывает неравенство (2.20). 
По лемме 23, если $|n|$ достаточно велико, то для каждого $\lambda \in U$ оператор $T=T(n, \lambda)$ ограничен и обратный $(1-T)^{-1}$ существует. Так как $E_{n}^{0}$ есть собственное подпространство оператора $L^{0}$, соответствующее $n^{2}$, то в силу леммы 21 мы имеем следующее: если $\lambda \in U$, то $\lambda \in \operatorname{Sp}(L)$ тогда и только тогда, когда $\lambda \in n^{2}+\operatorname{Sp}(S)$, где

$$
S=S_{\lambda}=P^{0}(1-T)^{-1} V P^{0}: E_{n}^{0} \rightarrow E_{n}^{0} .
$$

Другими словами, $\lambda \in U$ является собственным значением оператора $L$ тогда и только тогда, когда $z=\lambda-n^{2},|z| \leqslant|n| / 2$, есть собственное значение оператора $S$.

Пусть $\left(\begin{array}{ll}S^{11} & S^{12} \\ S^{21} & S^{22}\end{array}\right)$ будет матричным представлением двумерного оператора $S$ относительно базиса $e_{n}^{1}, e_{n}^{2}$; тогда

$$
S^{i j}=\left\langle S e_{n}^{j}, e_{n}^{i}\right\rangle, \quad i, j \in\{1,2\} .
$$

Рассмотрим уравнение для собственного значения оператора $S$ :

$$
\operatorname{det}\left|\begin{array}{cc}
S^{11}-z & S^{12} \\
S^{21} & S^{22}-z
\end{array}\right|=0
$$

Это есть основное уравнение в случае Хилла-Шрёдингера. Число $\lambda=n^{2}+z \in$ $U$ есть периодическое или антипериодическое собственное значение оператора $L^{0}+V$ тогда и только тогда, когда $z$ является решением для (2.23).

Наша следующая цель - дать явные формулы для матричных элементов $S^{i j}$. По лемме 23 , мы имеем $\left\|T_{n}\right\| \leqslant 1 / 2$ для $n \geqslant n_{0}(\|v\|)$, поэтому $\left(1-T_{n}\right)^{-1}=$ $\sum_{k=0}^{\infty} T_{n}^{k}$, так что

$$
S=\sum_{k=0}^{\infty} P_{n}^{0} T_{n}^{k} V P_{n}^{0}
$$

В силу (2.22),

$$
S^{i j}=\sum_{k=0}^{\infty} S_{k}^{i j}, \quad \text { где } \quad S_{k}^{i j}=\left\langle T_{n}^{k} V e_{n}^{j}, e_{n}^{i}\right\rangle .
$$

Таким образом, ввиду (2.18), (2.19) и (1.3), мы имеем

$$
S_{0}^{11}=\left\langle V e_{n}^{1}, e_{n}^{1}\right\rangle=V(0)=0, \quad S_{0}^{22}=\left\langle V e_{n}^{2}, e_{n}^{2}\right\rangle=V(0)=0
$$

и для каждого $k=1,2, \ldots$

$$
\begin{aligned}
S_{k}^{11} & =\sum_{j_{1}, \ldots, j_{k} \neq \pm n} \frac{V\left(-n-j_{1}\right) V\left(j_{1}-j_{2}\right) \cdots V\left(j_{k-1}-j_{k}\right) V\left(j_{k}+n\right)}{\left(n^{2}-j_{1}^{2}+z\right) \cdots\left(n^{2}-j_{k}^{2}+z\right)}, \\
S_{k}^{22} & =\sum_{j_{1}, \ldots, j_{k} \neq \pm n} \frac{V\left(n-j_{1}\right) V\left(j_{1}-j_{2}\right) \cdots V\left(j_{k-1}-j_{k}\right) V\left(j_{k}-n\right)}{\left(n^{2}-j_{1}^{2}+z\right) \cdots\left(n^{2}-j_{k}^{2}+z\right)} .
\end{aligned}
$$

Аналогично мы получаем:

$$
S_{0}^{12}=\left\langle V e_{n}^{2}, e_{n}^{1}\right\rangle=V(-2 n), \quad S_{0}^{21}=\left\langle V e_{n}^{1}, e_{n}^{2}\right\rangle=V(2 n)
$$


и для $k=1,2, \ldots$

$$
\begin{aligned}
S_{k}^{12} & =\sum_{j_{1}, \ldots, j_{k} \neq \pm n} \frac{V\left(-n-j_{1}\right) V\left(j_{1}-j_{2}\right) \cdots V\left(j_{k-1}-j_{k}\right) V\left(j_{k}-n\right)}{\left(n^{2}-j_{1}^{2}+z\right) \cdots\left(n^{2}-j_{k}^{2}+z\right)}, \\
S_{k}^{21} & =\sum_{j_{1}, \ldots, j_{k} \neq \pm n} \frac{V\left(n-j_{1}\right) V\left(j_{1}-j_{2}\right) \cdots V\left(j_{k-1}-j_{k}\right) V\left(j_{k}+n\right)}{\left(n^{2}-j_{1}^{2}+z\right) \cdots\left(n^{2}-j_{k}^{2}+z\right)} .
\end{aligned}
$$

Оператор $S$ зависит от $v, n \in \mathbb{N}$ и $\lambda$ (или $z=\lambda-n^{2}$ ), $|z| \leqslant|n| / 2$. Конечно, то же самое верно для его матрицы, т.е.

$$
S^{i j}=S^{i j}(v ; n, z), \quad S_{k}^{i j}=S_{k}^{i j}(v ; n, z) .
$$

ЛЕмма 24. (а) Для любого (комплекснозначного) потенииала $v$

$$
S^{11}(v ; n, z)=S^{22}(v ; n, z) .
$$

(b) Если v-вещественнозначный потенииал, то

$$
S^{12}(v ; n, z)=\overline{S^{21}(v ; n, \bar{z})} .
$$

ДокАзАТЕЛЬство. (а) В силу (2.26) и (2.27), замена индексов

$$
i_{s}=-j_{k+1-s}, \quad s=1, \ldots, k,
$$

показывает, что $S_{k}^{11}(-n, z)=S_{k}^{22}(n, z)$ для $k=1,2, \ldots$. Таким образом, ввиду (2.24) и (2.25), соотношение (2.31) выполняется.

(b) Если потенциал $v$ вещественнозначен, то для его коэффициентов Фурье мы имеем: $V(-m)=\overline{V(m)}$. В силу $(2.28)$,

$$
S_{0}^{12}(v ;-n, z)=V(-2 n)=\overline{V(2 n)}=\overline{S_{0}^{21}(v ; n, \bar{z})} .
$$

Более того, для каждого $k=1,2, \ldots$ замена индексов

$$
i_{s}=j_{k+1-s}, \quad s=1, \ldots, k,
$$

объясняет, что $S_{k}^{12}(-n, z)=\overline{S_{k}^{21}(n, \bar{z})}$. Таким образом, в силу $(2.24)$, соотношение (2.32) выполнено. Лемма 24 доказана.

Положим для каждого $n>n_{0}(\|v\|)$

$$
\alpha_{n}(v ; z)=S^{11}(v ; n, z), \quad \beta_{n}^{+}(v ; z)=S^{21}(v ; n, z), \quad \beta_{n}^{-}(v ; z)=S^{12}(v ; n, z) .
$$

2.3. Оценки $\alpha_{n}(v ; z)$ и $\beta_{n}^{ \pm}(v ; z)$ в случае операторов Хилла-Шрёдингера. Наш подход к нахождению хороших оценок параметров $\alpha$ и $\beta$ основан на следующем утверждении.

Лемма 25. Пусть $n \in \mathbb{N}$ зафиксировано, $H=\left(H_{i j}\right)$ - оператор ГилъбертаШмидта и $r \in \ell^{2}(2 \mathbb{Z}) ;$ тогда

$$
\sum_{i, j \neq \pm n}\left|\frac{r( \pm n-i) r( \pm n+j)}{n^{2}-j^{2}} H_{i j}\right| \leqslant \frac{2}{n}\|r\|^{2}\|H\|_{\mathrm{HS}} .
$$


ДоказАтеЛьство. Из неравенства Коши вытекает, что

$$
\sum_{i, j \neq \pm n}\left|\frac{r( \pm n-i) r( \pm n+j)}{n^{2}-j^{2}} H_{i j}\right| \leqslant(\sigma( \pm n))^{1 / 2}\|H\|_{\mathrm{HS}}
$$

где

$$
\sigma( \pm n)=\sum_{i, j \neq \pm n} \frac{|r( \pm n-i)|^{2}|r( \pm n+j)|^{2}}{\left|n^{2}-j^{2}\right|^{2}}
$$

Так как $|r( \pm n+j)| \leqslant\|r\|$, то по лемме 78 (см. дополнение) мы имеем

$$
\sigma( \pm n) \leqslant\left(\sum_{j \neq \pm n} \frac{1}{\left|n^{2}-j^{2}\right|^{2}}\right)\|r\|^{4} \leqslant \frac{4}{n^{2}}\|r\|^{4} .
$$

Это завершает доказательство.

Для любой последовательности $r \in \ell^{2}(2 \mathbb{Z})$ и $k, n \in \mathbb{N}$ положим

$$
\begin{aligned}
A_{k}(r ; n) & =\sum_{j_{1}, \ldots, j_{k} \neq \pm n} \frac{\left|r\left(n-j_{1}\right)\right|\left|r\left(j_{1}-j_{2}\right)\right| \cdots\left|r\left(j_{k}-n\right)\right|}{\left|n^{2}-j_{1}^{2}\right| \cdots\left|n^{2}-j_{k}^{2}\right|}, \\
B_{k}(r ; \pm n) & =\sum_{j_{1}, \ldots, j_{k} \neq \pm n} \frac{\left|r\left( \pm n-j_{1}\right)\right|\left|r\left(j_{1}-j_{2}\right)\right| \cdots\left|r\left(j_{k} \pm n\right)\right|}{\left|n^{2}-j_{1}^{2}\right| \cdots\left|n^{2}-j_{k}^{2}\right|}
\end{aligned}
$$

и

$$
A(r ; n)=\sum_{k=1}^{\infty} A_{k}(r ; n), \quad B(r ; \pm n)=\sum_{k=1}^{\infty} B_{k}(r ; \pm n) .
$$

В силу элементарного неравенства

$$
\frac{1}{\left|n^{2}+z-j^{2}\right|} \leqslant \frac{2}{\left|n^{2}-j^{2}\right|}, \quad j \in n+2 \mathbb{Z}, \quad j \neq \pm n, \quad|z| \leqslant n / 2,
$$

из $(2.26),(2.29)$ и (2.30) вытекает, что для $k \in \mathbb{N},|z| \leqslant n / 2$

$$
\left|S_{k}^{11}(v ; n, z)\right| \leqslant A_{k}(V ; n),\left|S_{k}^{12}(v ; n, z)\right| \leqslant B_{k}(V ;-n), \quad\left|S_{k}^{21}(v ; n, z)\right| \leqslant B_{k}(V ; n),
$$

где $V=(V(m))_{m \in 2 \mathbb{Z}}$ - последовательность коэффициентов Фурье потенциала $v(x)=\sum_{m \in 2 \mathbb{Z}} V(m) \exp (i m x)$. Поэтому в силу (2.33) мы имеем:

$$
\left|\alpha_{n}(v ; z)\right| \leqslant A(V ; n), \quad\left|\beta_{n}^{ \pm}(v ; z)-V( \pm 2 n)\right| \leqslant B(V ; \pm n) .
$$

Мы оцениваем сверху $A(r ; n)$ и $B(r ; \pm n)$, используя следующие формулы:

$$
\begin{gathered}
A(r ; n)=\left\langle\widehat{V} \widehat{D} \widehat{V} e_{n}, e_{n}\right\rangle+\left\langle\widehat{V} \widehat{D} \widehat{T}(1-\widehat{T})^{-1} \widehat{V} e_{n}, e_{n}\right\rangle, \\
B(r ; \pm n)=\left\langle\widehat{V} \widehat{D} \widehat{V} e_{\mp n}, e_{ \pm n}\right\rangle+\left\langle\widehat{V} \widehat{D} \widehat{T}(1-\widehat{T})^{-1} \widehat{V} e_{\mp n}, e_{ \pm n}\right\rangle,
\end{gathered}
$$

где $\widehat{V}, \widehat{D}, \widehat{T}$ обозначают соответственно операторы с матричными представлениями

$$
\begin{gathered}
\widehat{V}_{i j}=|r(i-j)|, \quad \widehat{D}_{i j}=\frac{1}{\left|n^{2}-j^{2}\right|} \delta_{i j}, \quad j \neq \pm n \\
\widehat{T}_{i j}=\frac{|r(i-j)|}{\left|n^{2}-j^{2}\right|}, \quad i, j \neq \pm n .
\end{gathered}
$$


ЛЕмма 26. Если $r \in \ell^{2}(2 \mathbb{Z})$, то для $|n|>6\|r\|$ выполнены неравенства

$$
\text { (a) } A(r ; n) \leqslant \frac{4}{n}\|r\|^{2}, \quad \text { (b) } B(r ; \pm n) \leqslant \frac{4}{n}\|r\|^{2} \text {. }
$$

ДоказАтельство. Доказательство (a) и (b) по существу одно и то же, так что мы дадим детали только в случае (а).

В силу (2.42), (2.44) и (2.45),

$$
A(r ; n)=\Sigma_{1}+\Sigma_{2},
$$

где

$$
\begin{aligned}
& \Sigma_{1}=A_{1}(r ; n)=\sum_{j \neq \pm n} \frac{|r(n-j) r(j+n)|}{\left|n^{2}-j^{2}\right|} \\
& \Sigma_{2}=\left\langle\widehat{V} \widehat{D} \widehat{T}(1-\widehat{T})^{-1} \widehat{V} e_{n}, e_{n}\right\rangle .
\end{aligned}
$$

По неравенству Коши и лемме 78 (см. дополнение) мы имеем:

$$
\Sigma_{1} \leqslant\left(\sum_{j \neq \pm n} \frac{|r(n-j)|^{2}}{\left|n^{2}-j^{2}\right|^{2}}\right)^{1 / 2}\|r\| \leqslant \frac{2}{n}\|r\|^{2} .
$$

Теперь, используя лемму 25, мы оценим сверху $\Sigma_{2}$. Матричные представления операторов $\widehat{V}, \widehat{D}$ и $\widehat{T}$, данные в $(2.44),(2.45)$, влекут, что $\Sigma_{2}$ имеет вид $(2.34)$, где

$$
H(n)=(1-\widehat{T}(n))^{-1} \widehat{T}(n), \quad \widehat{T}(n)=\widehat{V} Q_{n}^{0} \widehat{D}_{n} .
$$

По лемме $23, \widehat{T}=\widehat{T}(n)$ есть оператор Гильберта-Шмидта такой, что

$$
\|\widehat{T}\|_{\mathrm{HS}} \leqslant \frac{3}{n}\|r\|
$$

Следовательно, $\|\widehat{T}\|_{\mathrm{HS}} \leqslant 1 / 2$, если $n>6\|r\|$. Более того, $H(n)$ при $n>6\|r\|$ оказываются операторами Гильберта-Шмидта и мы имеем:

$$
H(n)=\sum_{k=1}^{\infty} \widehat{T}^{k}, \quad\|H(n)\|_{\mathrm{HS}} \leqslant \sum_{k=1}^{\infty} \| \widehat{T}_{\mathrm{HS}}^{k} \leqslant 1 .
$$

Таким образом, из леммы 25 вытекает:

$$
\Sigma_{2} \leqslant \frac{2}{n}\|r\|^{2} .
$$

В силу (2.47) и (2.48) выполнено неравенство $A(r ; n) \leqslant 4\|r\|^{2} / n$. Это завершает доказательство леммы 26.

ЛЕмма 27. При принятых выше обозначениях если $r=(r(m))_{m \in 2 \mathbb{Z}, a \omega}$ есть субмультипликативный вес на $2 \mathbb{Z}$, то

$$
B(r ; \pm n) \omega(2 n) \leqslant B(\omega r ; \pm n)
$$

əде $\omega r=(\omega(m) r(m))_{m \in 2 \mathbb{Z}}$. 
ДокАзАТЕЛЬСтво. Ввиду (2.39), достаточно показать, что

$$
B_{k}(r ; \pm n) \omega(2 n) \leqslant B_{k}(\omega r ; \pm n), \quad k \in \mathbb{N} .
$$

Так как $\omega$ - субмультипликативный вес, то для каждого $k$-набора индексов $j_{1}, \ldots, j_{k} \neq \pm n$

$$
\omega(-2 n)=\omega(2 n) \leqslant \omega\left( \pm n-j_{1}\right) \omega\left(j_{1}-j_{2}\right) \cdots \omega\left(j_{k} \pm n\right) .
$$

Следовательно,

$$
\begin{aligned}
& \left|r\left( \pm n-j_{1}\right)\right|\left|r\left(j_{1}-j_{2}\right)\right| \cdots\left|r\left(j_{k} \pm n\right)\right| \cdot \omega(2 n) \\
& \quad \leqslant\left|\omega\left( \pm n-j_{1}\right) r\left( \pm n-j_{1}\right)\right|\left|\omega\left(j_{1}-j_{2}\right) r\left(j_{1}-j_{2}\right)\right| \cdots\left|\omega\left(j_{k} \pm n\right) r\left(j_{k} \pm n\right)\right| .
\end{aligned}
$$

В силу (2.40) отсюда вытекает соотношение (2.49). Это завершает доказательство.

Ввиду (2.41), предыдущие леммы приводят нас к следующему утверждению.

ПредлОЖениЕ 28. (а) Если $v \in L^{2}([0, \pi])$, то для $|n|>n_{0}(\|v\|) u|z| \leqslant|n| / 2$

$$
\left|\alpha_{n}(v ; z)\right| \leqslant \frac{4}{n}\|v\|^{2}, \quad\left|\beta_{n}^{ \pm}(v ; z)-v_{ \pm n}\right| \leqslant \frac{4}{n}\|v\|^{2} .
$$

(b) Eсли $\Omega=(\Omega(m))_{m \in \mathbb{Z}}-$ субмультипликативный вес, а $v \in H(\Omega)$, то для $|n|>n_{0}\left(\|v\|_{\Omega}\right) u|z| \leqslant|n| / 2$

$$
\left|\beta_{n}^{ \pm}(v ; z)-v_{ \pm n}\right| \cdot \Omega(n) \leqslant \frac{4}{n}\|v\|_{H(\Omega)}^{2},
$$

где $v_{ \pm n}-( \pm n)$-коэфбициенты Фуръе потенциала $v(x)=\sum_{m \in \mathbb{Z}} v_{m} \exp ( \pm 2 i m x)$.

ДокАЗАТЕльство. В силу (2.41) неравенство (2.51) вытекает из леммы 26; это доказывает часть (a).

Чтобы доказать (b), рассмотрим вес $\omega=(\omega(m))_{m \in 2 \mathbb{Z}}$, где $\omega(m)=\Omega(m / 2)$. Пусть $V=(V(m))_{m \in 2 \mathbb{Z}}$ - последовательность коэффициентов Фурье потенциала

$$
v(x)=\sum_{m \in 2 \mathbb{Z}} V(m) e^{i m x}=\sum_{k \in \mathbb{Z}} v_{k} e^{i 2 k x} .
$$

Тогда $V \in \ell^{2}(\omega, 2 \mathbb{Z})$ и

$$
\|V\|_{\omega}^{2}=\sum_{m \in 2 \mathbb{Z}}|V(m)|^{2}(\omega(m))^{2}=\sum_{k \in \mathbb{Z}}\left|v_{k}\right|^{2}(\Omega(k))^{2}=\|v\|_{H(\Omega)}^{2} .
$$

В силу (2.41) и леммы 27 ,

$$
\left|\beta_{n}^{ \pm}(v ; z)-v_{ \pm n}\right| \cdot \Omega(n) \leqslant B(V ; \pm n) \omega( \pm 2 n) \leqslant B(\omega \cdot V ; \pm n) .
$$

Теперь, применяя лемму 26 к последовательности $r=\omega \cdot V$, получаем

$$
B(\omega \cdot V ; \pm n) \leqslant \frac{4}{n}\|\omega \cdot V\|^{2}=\frac{4}{n}\|V\|_{\omega}^{2}=\frac{4}{n}\|v\|_{H(\Omega)}^{2} ;
$$

это завершает доказательство. 
Оценки параметров $\alpha_{n}$ и $\widetilde{\beta}_{n}^{ \pm}$, полученные в предложении 28, приводят к оценкам производных $\partial \alpha_{n} / \partial z$ и $\partial \beta_{n}^{ \pm} / \partial z$.

ЛЕмма 29. Для $|n|>n_{0}(v) u|z| \leqslant|n| / 4$ мъ имеем:

$$
\left|\frac{\partial \alpha_{n}}{\partial z}(v ; z)\right| \leqslant \frac{8}{n^{2}}\|v\|^{2}, \quad\left|\frac{\partial \beta_{n}^{ \pm}}{\partial z}(v ; z)\right| \leqslant \frac{8}{n^{2}}\|v\|^{2} .
$$

ДокАЗАТЕЛЬство. Оба параметра $\alpha_{n}$ и $\beta_{n}^{ \pm}$зависят аналитически от $z$, если $|z| \leqslant n / 2$, и ограничены в том же круге величиной $4\|v\|^{2} / n$, как это следует из предложения 28. Тогда неравенства Коши для их первых производных в круге $|z| \leqslant|n| / 4$ приводят к соотношению (2.53).

2.4. Основное уравнение: случай операторов Дирака. По локализационной теореме 17 , для $|n|>N_{*}(v)$ периодический оператор Дирака

$$
L=L^{0}+v, \quad v=\left(\begin{array}{cc}
0 & P(x) \\
Q(x) & 0
\end{array}\right),
$$

имеет в точности два (с учетом их кратности) периодических, если $n$ четно, или антипериодических, если $n$ нечетно, собственных значения в круге $U=D(n, r)$ с центром $n$ и радиусом $r=1 / 2$.

Зафиксируем $n>N_{*}(v)$. Пусть $\mathbb{H}=\left(L^{2}(I)\right)^{2}$, и пусть $E_{n}^{0}=\left[e_{n}^{1}, e_{n}^{2}\right]-$ собственное подпространство оператора $L^{0}$, соответствующее $n$, а $P_{n}^{0}$ - ортогональный проектор на $E_{n}^{0}$. Положим $Q_{n}^{0}=1-P_{n}^{0}$ и обозначим через $\mathbb{H}(n)$ область значений проектора $Q_{n}^{0}$. Ограничение $\lambda-L^{0}$ на $\mathbb{H}(n)$ обратимо. Обозначим через $D=D_{n}: \mathbb{H}(n) \rightarrow \mathbb{H}(n)$ обратный к нему оператор и положим

$$
T_{n}=V D Q_{n}^{0}: \mathbb{H} \rightarrow \mathbb{H} .
$$

Для $|n| \geqslant N_{*}(v)$ мы имеем: $\left\|T_{n}\right\|^{2}<1$, если $\lambda \in U$ (см. ниже лемму 33); это обеспечивает существование обратного оператора $\left(1-T_{n}\right)^{-1}$. Таким образом, мы можем применять лемму 21.

Так как $E_{n}^{0}$ - собственное подпространство оператора $L^{0}$, соответствующее $n$, то по лемме 21 мы имеем: если $\lambda \in U$, то $\lambda \in \operatorname{Sp}\left(L^{0}+V\right)$ тогда и только тогда, когда $\lambda \in n+\operatorname{Sp}(S)$, где

$$
S=P^{0}\left(1-T_{n}\right)^{-1} V P^{0}: E_{n}^{0} \rightarrow E_{n}^{0} .
$$

Другими словами, $\lambda \in U$ есть собственное значение оператора $L^{0}+V$ (периодическое или антипериодическое) тогда и только тогда, когда $z=\lambda-n,|z| \leqslant 1 / 2$, является собственным значением оператора $S$.

Пусть $\left(\begin{array}{ll}S^{11} & S^{12} \\ S^{21} & S^{22}\end{array}\right)$ - это матричное представление двумерного оператора $S$ относительно базиса $e_{n}^{1}, e_{n}^{2}$; тогда

$$
S^{11}=\left\langle S e_{n}^{1}, e_{n}^{1}\right\rangle, \quad S^{22}=\left\langle S e_{n}^{2}, e_{n}^{2}\right\rangle, \quad S^{12}=\left\langle S e_{n}^{2}, e_{n}^{1}\right\rangle, \quad S^{21}=\left\langle S e_{n}^{1}, e_{n}^{2}\right\rangle .
$$

Рассмотрим уравнение для собственного значения оператора $S$ :

$$
\operatorname{det}\left|\begin{array}{cc}
S^{11}-z & S^{12} \\
S^{21} & S^{22}-z
\end{array}\right|=0
$$


Это основное уравнение в случае Дирака. Число $\lambda \in U, \lambda=n+z$, является периодическим или антипериодическим собственным значением оператора $L^{0}+V$ тогда и только тогда, когда $z$ является решением уравнения (2.58).

Наша следующая цель - дать явные формулы для матричных элементов $S^{i j}$. Пусть $\mathbb{H}^{1}$ и $\mathbb{H}^{2}$ - это подпространства в $\mathbb{H}$, порожденные соответственно системой $\left\{e_{m}^{1}, m \in \mathbb{Z}\right\}$ и системой $\left\{e_{m}^{2}, m \in \mathbb{Z}\right\}$, и пусть $\mathbb{H}^{1}(n)$ и $\mathbb{H}^{2}(n)$ будут соответственно пересечениями этих подпространств с $\mathbb{H}(n)$. Тогда $\mathbb{H}=\mathbb{H}^{1} \oplus \mathbb{H}^{2}$, так что каждый оператор $B: \mathbb{H} \rightarrow \mathbb{H}$ можно отождествить с блочно-операторной $(2 \times 2)$-матрицей $\left(B^{i j}\right)$, где $B^{i j}: \mathbb{H}^{j} \rightarrow \mathbb{H}^{i}, i, j=1,2$. Если мы рассмотрим матричное представление для $B$ в базисе $\left\{e_{2 k}^{1}, e_{2 k}^{2}, k \in \mathbb{Z}\right\}$ (или $\left\{e_{2 k+1}^{1}, e_{2 k+1}^{2}, k \in \mathbb{Z}\right\}$ ), то эта матрица сама объединяет в себе матричные представления блоков $B^{i j}$. Конечно, аналогичные замечания могут быть сделаны для операторов, действующих в $\mathbb{H}(n)$.

Напомним, что

$$
P(x)=\sum_{m \in \mathbb{Z}} p(m) e^{i m x} \quad \text { и } \quad Q(x)=\sum_{m \in \mathbb{Z}} q(m) e^{i m x},
$$

где $p(m)=q(m)=0$, если $m$ нечетно. Легко видеть, что оператор $V$ имеет матричное представление

$$
V=\left(\begin{array}{cc}
0 & V^{12} \\
V^{21} & 0
\end{array}\right), \quad V_{k m}^{12}=p(-k-m), \quad V_{k m}^{21}=q(k+m),
$$

а матричное представление для $D=D_{n}$ есть

$$
D_{n}=\left(\begin{array}{cc}
D_{n}^{11} & 0 \\
0 & D_{n}^{22}
\end{array}\right), \quad\left(D_{n}^{11}\right)_{k m}=\left(D_{n}^{22}\right)_{k m}=\frac{\delta_{k m}}{\pi(n-k)+z} .
$$

В силу (2.60) и (2.61), легко видеть, что оператор (2.55) имеет матричное представление

$$
T_{n}=\left(\begin{array}{cc}
0 & T_{n}^{12} \\
T_{n}^{21} & 0
\end{array}\right)
$$

где

$$
\left(T_{n}^{12}\right)_{k m}=\frac{p(-k-m)}{n-k+z}, \quad\left(T_{n}^{21}\right)_{k m}=\frac{q(k+m)}{n-k+z}, \quad k, m \in \mathbb{Z}, \quad k \neq n .
$$

Нам нужно также знать матричное представление его квадрата $T_{n}^{2}$. Из (2.62) и (2.63) вытекает, что

где

$$
T_{n}^{2}=\left(\begin{array}{cc}
T_{n}^{12} T_{n}^{21} & 0 \\
0 & T_{n}^{21} T_{n}^{12}
\end{array}\right)
$$

$$
\begin{aligned}
\left(T_{n}^{12} T_{n}^{21}\right)_{k m} & =\sum_{j \neq n} \frac{p(-k-j) q(j+m)}{(n-k+z)(n-j+z)}, \\
\left(T_{n}^{21} T_{n}^{12}\right)_{k m} & =\sum_{j \neq n} \frac{q(k+j) p(-j-m)}{(n-k+z)(n-j+z)},
\end{aligned}
$$


Теперь из (2.55) и (2.56) вытекает, что

$$
S=\sum_{k=0}^{\infty} P_{n}^{0} T_{n}^{k} P_{n}^{0} V
$$

следовательно, ввиду (2.57), мы имеем

$$
S^{i j}=\left\langle S e_{n}^{j}, e_{n}^{i}\right\rangle=\sum_{k=0}^{\infty} S_{k}^{i j},
$$

где

$$
S_{k}^{i j}=\left\langle T_{n}^{k} V e_{n}^{j}, e_{n}^{i}\right\rangle, \quad k=0,1,2, \ldots
$$

Из (2.60) и (2.63)-(2.65) вытекает, что

$$
\begin{aligned}
T_{n}^{2 \nu} V & =\left(\begin{array}{cc}
0 & \left(T_{n}^{21} T_{n}^{12}\right)^{\nu} V^{12} \\
\left(T_{n}^{12} T_{n}^{21}\right)^{\nu} V^{21} & 0
\end{array}\right), \\
T_{n}^{2 \nu+1} V & =\left(\begin{array}{cc}
T_{n}^{21}\left(T_{n}^{21} T_{n}^{12}\right)^{\nu} V^{12} & 0 \\
0 & T_{n}^{12}\left(T_{n}^{12} T_{n}^{21}\right)^{\nu} V^{21}
\end{array}\right) .
\end{aligned}
$$

Легко видеть, что

$$
\left\langle T_{n}^{2 \nu} V e_{n}^{i}, e_{n}^{i}\right\rangle=0, \quad i=1,2, \quad \nu=0,1,2, \ldots ;
$$

следовательно, в силу (2.57)-(2.69) мы получаем:

$$
S^{11}=\sum_{\nu=0}^{\infty} S_{2 \nu+1}^{11}, \quad S^{22}=\sum_{\nu=0}^{\infty} S_{2 \nu+1}^{22},
$$

где

$$
\begin{aligned}
& S_{2 \nu+1}^{11}=\left\langle T_{n}^{2 \nu+1} V e_{n}^{1}, e_{n}^{1}\right\rangle=\left\langle T_{n}^{21}\left(T_{n}^{21} T_{n}^{12}\right)^{\nu} V^{12} e_{n}^{1}, e_{n}^{1}\right\rangle \\
& =\sum_{j_{0}, j_{1}, \ldots, j_{2 \nu} \neq n} \frac{p\left(-n-j_{0}\right) q\left(j_{0}+j_{1}\right) p\left(-j_{1}-j_{2}\right) q\left(j_{2}+j_{3}\right) \cdots p\left(-j_{2 \nu-1}-j_{2 \nu}\right) q\left(j_{2 \nu}+n\right)}{\left(n-j_{0}+z\right)\left(n-j_{1}+z\right) \cdots\left(n-j_{2 \nu-1}+z\right)\left(n-j_{2 \nu}+z\right)} \\
& S_{2 \nu+1}^{22}=\left\langle T_{n}^{2 \nu+1} V e_{n}^{2}, e_{n}^{2}\right\rangle=\left\langle T_{n}^{12}\left(T_{n}^{12} T_{n}^{21}\right)^{\nu} V^{21} e_{n}^{2}, e_{n}^{2}\right\rangle \\
& =\sum_{i_{0}, i_{1}, \ldots, i_{2 \nu} \neq n} \frac{q\left(n+i_{0}\right) p\left(-i_{0}-i_{1}\right) q\left(i_{1}+i_{2}\right) p\left(-i_{2}-i_{3}\right) \cdots q\left(j_{2 \nu-1}+j_{2 \nu}\right) p\left(-j_{2 \nu}-n\right)}{\left(n-i_{0}+z\right)\left(n-i_{1}+z\right) \cdots\left(n-i_{2 \nu-1}+z\right)\left(n-i_{2 \nu}+z\right)}
\end{aligned}
$$

Аналогично мы получаем формулы для элементов $S^{12}$ и $S^{21}$. Действительно,

$$
\left\langle T_{n}^{2 \nu+1} V e_{n}^{2}, e_{n}^{1}\right\rangle=0, \quad\left\langle T_{n}^{2 \nu+1} V e_{n}^{1}, e_{n}^{2}\right\rangle=0, \quad \nu=0,1,2, \ldots,
$$

и поэтому из $(2.57),(2.66)-(2.69)$ вытекает, что

$$
S^{12}=\sum_{\nu=0}^{\infty} S_{2 \nu}^{12}, \quad S^{21}=\sum_{\nu=0}^{\infty} S_{2 \nu}^{21},
$$


где

$$
S_{0}^{12}=\left\langle V e_{n}^{2}, e_{n}^{1}\right\rangle=p(-2 n), \quad S_{0}^{21}=\left\langle V e_{n}^{1}, e_{n}^{2}\right\rangle=q(2 n)
$$

и для $\nu=1,2, \ldots$

$$
\begin{aligned}
& S_{2 \nu}^{12}=\left\langle T_{n}^{2 \nu} V e_{n}^{2}, e_{n}^{1}\right\rangle=\left\langle\left(T_{n}^{12} T_{n}^{21}\right)^{\nu} V^{21} e_{n}^{2}, e_{n}^{1}\right\rangle \\
& =\sum_{j_{1}, \ldots, j_{2 \nu} \neq n} \frac{p\left(-n-j_{1}\right) q\left(j_{1}+j_{2}\right) p\left(-j_{2}-j_{3}\right) q\left(j_{3}+j_{4}\right) \cdots q\left(j_{2 \nu-1}+j_{2 \nu}\right) p\left(-j_{2 \nu}-n\right)}{\left(n-j_{1}+z\right)\left(n-j_{2}+z\right) \cdots\left(n-j_{2 \nu-1}+z\right)\left(n-j_{2 \nu}+z\right)} \\
& S_{2 \nu}^{21}=\left\langle T_{n}^{2 \nu} V e_{n}^{1}, e_{n}^{2}\right\rangle=\left\langle\left(T_{n}^{21} T_{n}^{12}\right)^{\nu} V^{12} e_{n}^{1}, e_{n}^{2}\right\rangle \\
& =\sum_{j_{1}, \ldots, j_{2 \nu} \neq n} \frac{q\left(n+j_{1}\right) p\left(-j_{1}-j_{2}\right) q\left(j_{2}+j_{3}\right) p\left(-j_{3}-j_{4}\right) \cdots p\left(-j_{2 \nu-1}-j_{2 \nu}\right) q\left(j_{2 \nu}+n\right)}{\left(n-j_{1}+z\right)\left(n-j_{2}+z\right) \cdots\left(n-j_{2 \nu-1}+z\right)\left(n-j_{2 \nu}+z\right)} .
\end{aligned}
$$

Лемма 30. (а) Для любых функицй $P, Q$ в потенииале $v$ мы имеем соотношение

$$
S^{11}(n, z)=S^{22}(n, z) .
$$

(b) Если же $Q(x)=\overline{P(x)}$, mо

$$
S^{12}(n, z)=\overline{S^{21}(n, \bar{z})}
$$

ДокАЗАТЕЛЬСтво. (а) Изменим индексы суммирования в (2.73), полагая

$$
j_{s}=i_{2 \nu-s}, \quad s=0,1, \ldots, 2 \nu .
$$

Мы получаем в силу (2.72), что

$$
S_{2 \nu+1}^{22}=S_{2 \nu+1}^{11}, \quad \nu=0,1,2, \ldots
$$

и поэтому, в силу $(2.71)$, мы имеем $S^{22}=S^{11}$.

(b) Если же $Q(x)=\overline{P(x)}$, то $q(m)=\overline{p(-m)}$ для всех $m \in \mathbb{Z}$, и поэтому $(2.75)$, $(2.76)$ и $(2.77)$ влекут, что $\overline{S_{2 \nu}^{21}(n, \bar{z})}=S_{2 \nu}^{12}(n, z)$ для всех $\nu=0,1,2, \ldots$, так что (2.74) приводит нас к (2.79). Лемма 30 доказана.

Далее, положим

$$
\alpha_{n}(v ; z):=S^{11}(n, z), \quad \beta_{n}^{+}(v ; z):=S^{21}(v ; n, z), \quad \beta_{n}^{-}(v ; z):=S^{12}(v ; n, z) .
$$

2.5. Оценки параметров $\alpha_{n}(v ; z)$ и $\beta_{n}^{ \pm}(v ; z)$ в случае операторов Дирака. Следующие леммы аналогичны лемме 25 , что была использована в случае операторов Хилла-Шрёдингера.

Лемма 31. Пусть $n \in \mathbb{Z} \backslash\{0\}$ зафиксировано $u r \in \ell^{2}(2 \mathbb{Z})$, a $H=\left(H_{i j}\right)_{i, j \in n+2 \mathbb{Z}}$ есть оператор Гильберта-Шмидта. Тогда

$$
\sum_{i, j \neq n}\left|\frac{r(n+i) r(n+j)}{n-i} H_{i j}\right| \leqslant\left(\|r\| \mathscr{E}_{|n|}(r)+\frac{\|r\|^{2}}{\sqrt{|n|}}\right)\|H\|_{\mathrm{HS}},
$$

где суммирование идет по парам $i, j \in n+2 \mathbb{Z} u$

$$
\mathscr{E}_{|n|}(r)=\left(\sum_{|m| \geqslant|n|}|r(m)|^{2}\right)^{1 / 2} \text {. }
$$


ДоказАТЕЛЬство. Из неравенства Коши вытекает, что

$$
\sum_{i, j \neq n}\left|\frac{r(n+i) r(n+j)}{n-i} H_{i j}\right| \leqslant(\sigma(n))^{1 / 2}\|H\|_{\mathrm{HS}}
$$

где

$$
\sigma(n)=\sum_{i, j \neq n} \frac{|r(n+i)|^{2}|r(n+j)|^{2}}{|n-i|^{2}} \leqslant\|r\|^{2} \sum_{i \neq n} \frac{|r(n+i)|^{2}}{|n-i|^{2}} .
$$

Если $n>0$, то

$$
\begin{aligned}
\sum_{i \neq n} \frac{|r(n+i)|^{2}}{|n-i|^{2}} & =\sum_{i \geqslant 0}+\sum_{i<0} \leqslant \sum_{m \geqslant n}|r(m)|^{2}+\left(\sum_{k>n} \frac{1}{k^{2}}\right) \cdot\|r\|^{2} \\
& \leqslant(\mathscr{E}|n|(r))^{2}+\|r\|^{2} /|n| .
\end{aligned}
$$

Случай, когда $n<0$, можно рассмотреть тем же самым способом, что приводит к таким же оценкам. Это завершает доказательство.

Вес $\Omega$ назовем медленно растущим, если

$$
M:=\sup _{n} \frac{\Omega(2 n)}{\Omega(n)}<\infty .
$$

Лемма 32. Пусть $\omega=(\omega(m))_{m \in 2 \mathbb{Z}}-$ такой медленно растущий вес, что

$$
\exists C_{1}: \quad \omega(m) \leqslant C_{1}|m| \quad \forall m \in 2 \mathbb{Z},
$$

и пусть $r=(r(m))_{m \in 2 \mathbb{Z}} \in \ell^{2}(\omega, 2 \mathbb{Z})$. Пусть $\bar{r}=(\bar{r}(m))_{m \in 2 \mathbb{Z}}$ определено соотношением

$$
\bar{r}(m)=\omega(m) r(m), \quad m \in 2 \mathbb{Z} .
$$

Предположим, что $H(n)=\left(H_{i j}(n)\right)_{i, j \in n+2 \mathbb{Z}},|n| \geqslant n_{0},-$ такое семейство операторов Гильберта-Шмидта, что

$$
H_{*}:=\sup _{|n| \geqslant n_{0}}\|H(n)\|_{\mathrm{HS}}<\infty .
$$

Тогда для $N>n_{0}$

$$
\sum_{|n|>N}(\omega(2 n))^{2}\left(\sum_{i, j \neq n}\left|\frac{r(n+i) r(n+j)}{|n-i||n-j|} H_{i j}(n)\right|\right)^{2} \leqslant C H_{*}^{2}\left(\frac{\left(\mathscr{E}_{N}(\bar{r})\right)^{4}}{(\omega(2 N))^{2}}+\frac{\|r\|^{4}}{N}\right)
$$

где $C=C(\omega)$.

ДокАзАТЕльство. По неравенству Коши, левая часть в (2.85) (обозначим ее $\Sigma)$ не превосходит

$$
\begin{gathered}
\sum_{|n|>N}(\omega(2 n))^{2}\left(\sum_{i, j \neq n} \frac{|r(n+i)|^{2}|r(n+j)|^{2}}{|n-i|^{2}|n-j|^{2}}\right) \cdot\left(\sum_{i, j}\left|H_{i j}(n)\right|^{2}\right) \\
\leqslant \sum_{|n|>N}(\omega(2 n))^{2}\left(\sum_{i \neq n} \frac{|r(n+i)|^{2}}{|n-i|^{2}}\right)^{2} \cdot H_{*}^{2} .
\end{gathered}
$$


Пусть $\nu=n-i$; тогда мы имеем:

$$
\left(\sum_{i \neq n} \frac{|r(n+i)|^{2}}{|n-i|^{2}}\right)^{2}=\left(\sum_{\nu \neq 0} \frac{|r(2 n-\nu)|^{2}}{\nu^{2}}\right)^{2} \leqslant 2\left(\sum_{0<|\nu| \leqslant|n|} \cdots\right)^{2}+2\left(\sum_{|\nu|>|n|} \ldots\right)^{2} .
$$

Так как $\omega$ - медленно растущий вес, то существует постоянная $C_{0}>0$ такая, что

$$
\omega(2 m) \leqslant C_{0} \omega(m), \quad m \in 2 \mathbb{Z} .
$$

Если $|\nu| \leqslant|n|$, то $|2 n-\nu| \geqslant 2|n|-|\nu| \geqslant|n|$. Но тогда, в силу (2.84) и (2.87),

$$
\sum_{0<|\nu| \leqslant|n|} \frac{|r(2 n-\nu)|^{2}}{\nu^{2}}=\sum_{0<|\nu| \leqslant|n|} \frac{|\bar{r}(2 n-\nu)|^{2}}{(\omega(2 n-\nu))^{2} \nu^{2}} \leqslant \frac{C_{0}^{2}}{(\omega(2 n))^{2}} \sum_{0<|\nu| \leqslant|n|} \frac{|\bar{r}(2 n-\nu)|^{2}}{\nu^{2}} .
$$

По неравенству Коши,

$$
\begin{aligned}
\left(\sum_{0<|\nu| \leqslant|n|} \frac{|\bar{r}(2 n-\nu)|^{2}}{\nu^{2}}\right)^{2} & \leqslant \sum_{0<|\nu| \leqslant|n|}|\bar{r}(2 n-\nu)|^{2} \sum_{\substack{0<|\nu| \leqslant|n| \\
\nu^{4}}} \frac{|\bar{r}(2 n-\nu)|^{2}}{|\bar{r}(2 n-\nu)|^{2}} \\
& \leqslant\left(\mathscr{E}_{|n|}(\bar{r})\right)^{2} \sum_{0<|\nu| \leqslant|n|} \frac{\mid \nu^{4}}{}
\end{aligned}
$$

Следовательно, мы имеем:

$$
\left(\sum_{0<|\nu| \leqslant|n|} \frac{|r(2 n-\nu)|^{2}}{\nu^{2}}\right)^{2} \leqslant \frac{C_{0}^{4}}{(\omega(2 n))^{4}}\left(\mathscr{E}_{|n|}(\bar{r})\right)^{2} \sum_{0<|\nu| \leqslant|n|} \frac{|\bar{r}(2 n-\nu)|^{2}}{\nu^{4}} .
$$

Но, с другой стороны,

$$
\sum_{|\nu|>|n|} \frac{|r(2 n-\nu)|^{2}}{\nu^{2}} \leqslant \frac{1}{n^{2}} \sum_{|\nu|>|n|}|r(2 n-\nu)|^{2} \leqslant \frac{1}{n^{2}}\|r\|^{2},
$$

и поэтому

$$
\left(\sum_{i \neq n} \frac{|r(n+i)|^{2}}{|n-i|^{2}}\right)^{2} \leqslant \frac{2 C_{0}^{4}(\mathscr{E}|n|(r))^{2}}{(\omega(2 n))^{4}} \sum_{0<|\nu| \leqslant|n|} \frac{|\bar{r}(2 n-\nu)|^{2}}{\nu^{4}}+\frac{2}{n^{4}}\|r\|^{4}
$$

Таким образом, в силу $(2.86)$ и $(2.88)$, сумма $\Sigma$ в (2.85) не превосходит

$$
\frac{2 C_{0}^{2} H_{*}^{2}\left(\mathscr{E}_{N}(\bar{r})\right)^{2}}{(\omega(2 N))^{2}} \sum_{|n|>N} \sum_{0<|\nu| \leqslant|n|} \frac{|\bar{r}(2 n-\nu)|^{2}}{\nu^{4}}+2 H_{*}^{2}\|r\|^{4} \sum_{|n|>N} \frac{(\omega(2 n))^{2}}{n^{4}} .
$$

Изменим теперь индексы суммирования в двойной сумме, полагая

$$
\nu=\nu, \quad k=2 n-\nu .
$$

Так как из $|\nu| \leqslant|n|,|n|>N$ вытекает, что $|k|=|2 n-\nu| \geqslant 2|n|-|\nu| \geqslant|n|>N$, то мы имеем:

$$
\sum_{|n|>N} \sum_{0<|\nu| \leqslant|n|} \frac{|\bar{r}(2 n-\nu)|^{2}}{\nu^{4}} \leqslant \sum_{\nu \neq 0,|k|>N} \frac{|\bar{r}(k)|^{2}}{\nu^{4}} \leqslant C_{2}\left(\mathscr{E}_{N}(\bar{r})\right)^{2},
$$


где $C_{2}=\sum_{\nu \neq 0} 1 / \nu^{4}$. С другой стороны, в силу (2.83), мы имеем:

$$
\sum_{|n|>N} \frac{(\omega(2 n))^{2}}{n^{4}} \leqslant \sum_{|n|>N} \frac{\left(2 C_{1}\right)^{2}}{n^{2}} \leqslant \frac{\widetilde{C}}{N}
$$

где $\widetilde{C}=\widetilde{C}(\omega)$. Эти оценки завершают доказательство леммы 32 .

Для $r=(r(m))_{m \in 2 \mathbb{Z}}$ положим

$$
A(r ; n)=\sum_{\nu=0}^{\infty} A_{2 \nu+1}(r ; n), \quad B(r ; n)=\sum_{\nu=1}^{\infty} B_{2 \nu}(r ; n),
$$

где

$$
\begin{aligned}
A_{2 \nu+1}(r ; n) & =\sum_{j_{0}, j_{1}, \ldots, j_{2 \nu} \neq n} \frac{r\left(-n-j_{0}\right) r\left(j_{0}+j_{1}\right) \cdots r\left(-j_{2 \nu-1}-j_{2 \nu}\right) r\left(j_{2 \nu}+n\right)}{\left|n-j_{0}\right| \cdot\left|n-j_{1}\right| \cdots\left|n-j_{2 \nu-1}\right| \cdot\left|n-j_{2 \nu}\right|}, \\
B_{2 \nu}(r ; n) & =\sum_{j_{1}, \ldots, j_{2 \nu} \neq n} \frac{r\left(n+j_{1}\right) r\left(-j_{1}-j_{2}\right) \cdots r\left(-j_{2 \nu-1}-j_{2 \nu}\right) r\left(j_{2 \nu}+n\right)}{\left|n-j_{1}\right| \cdot\left|n-j_{2}\right| \cdots\left|n-j_{2 \nu-1}\right| \cdot\left|n-j_{2 \nu}\right|} .
\end{aligned}
$$

Пусть

$$
r=(r(m))_{m \in \mathbb{Z}}, \quad r(m)=\max \{|p( \pm m)|,|q( \pm m)|\}
$$

тогда

$$
\frac{1}{2}\|v\| \leqslant\|r\| \leqslant 2\|v\|=2\left(\sum\left(|p(k)|^{2}+|q(k)|^{2}\right)\right)^{1 / 2} .
$$

Ввиду (2.72), (2.73), (2.90) и (2.92), элементарное неравенство

$$
\frac{1}{|n-j+z|} \leqslant \frac{2}{|n-j|} \quad \text { для } \quad j \neq n, \quad|z| \leqslant \frac{1}{2}
$$

влечет за собой, что

$$
\left|S_{2 \nu+1}^{i i}(v ; n, z)\right| \leqslant A_{2 \nu+1}(r ; n), \quad i=1,2, \quad \nu=0,1,2, \ldots .
$$

Следовательно, в силу (2.71), (2.80), (2.89), мы имеем:

$$
\left|\alpha_{n}(v ; z)\right|=\left|S^{11}(v ; n, z)\right| \leqslant A(r, n), \quad \text { если }|z| \leqslant 1 / 2 .
$$

Аналогично, $(2.76),(2.77)$ и (2.91)-(2.94) приводят к соотношениям

$$
\left|S^{12}(v ; n, z)\right| \leqslant B(r ; n), \quad\left|S^{21}(v ; n, z)\right| \leqslant B(r ; n) \quad \text { для } \quad|z| \leqslant 1 / 2 .
$$

Поэтому, в силу $(2.74),(2.80),(2.89)$ и $(2.97)$, мы получаем:

$$
\left|\beta_{n}^{-}(v ; z)-p(-2 n)\right|=\left|S^{12}(v ; n, z)-p(-2 n)\right| \leqslant B(r ; n)
$$

и

$$
\left|\beta_{n}^{+}(v ; z)-q(2 n)\right|=\left|S^{21}(v ; n, z)-q(2 n)\right| \leqslant B(r ; n) .
$$


Теперь наша цель - дать оценки сверху для $A(r ; n)$ и $B(r ; n)$. Для этого мы рассмотрим в $\left(\ell^{2}(\mathbb{Z})\right)^{2}$ операторы

$$
\begin{array}{rlrl}
\widehat{V} & =\left(\begin{array}{cc}
0 & \widehat{V}^{12} \\
\widehat{V}^{21} & 0
\end{array}\right), & \widehat{V}_{i j}^{12} & =r(-i-j), \quad \widehat{V}_{i j}^{21}=r(i+j) ; \\
\widehat{D} & =\left(\begin{array}{cc}
\widehat{D}^{11} & 0 \\
0 & \widehat{D}^{22}
\end{array}\right), & \widehat{D}_{i j}^{11} & =\widehat{D}_{i j}^{22}=\frac{\delta_{k m}}{|n-k|}, \quad k, m \neq n ; \\
\widehat{T} & =\widehat{V} \widehat{D} Q_{n}^{0} .
\end{array}
$$

Оператор $\widehat{T}$ доминирует оператор $T$ в том смысле, что каждый элемент в матричном представлении оператора $T$ не превосходит по абсолютной величине соответствующего элемента в матричном представлении оператора $\widehat{T}$. Поэтому мы можем оценить сверху норму Гильберта-Шмидта оператора $T$ нормой Гильберта-Шмидта оператора $\widehat{T}$.

ЛЕмма 33. При принятых выше обозначениях оператор $\widehat{T}^{2}$ лежит в классе Гильберта-Шмидта и

$$
\left\|\widehat{T}^{2}\right\|_{\mathrm{HS}} \leqslant C\left(\mathscr{E}_{|n|}(r)+\frac{\|r\|}{\sqrt{|n|+1}}\right) \cdot\|r\|
$$

где $C>0$ - абсолютная постоянная.

ДокАЗАТЕЛЬСтво. В силу (2.102) мы имеем:

$$
\widehat{T}^{2}=\widehat{V} \cdot G, \quad \text { где } \quad G:=\widehat{D} Q_{n}^{0} \widehat{V} \widehat{D} Q_{n}^{0}: \mathbb{H}(n) \rightarrow \mathbb{H}(n) .
$$

Матричное представление для $G$ имеет вид

$$
G=\left(\begin{array}{cc}
0 & G^{12} \\
G^{21} & 0
\end{array}\right), \quad G_{j k}^{12}=G_{j k}^{21}=\frac{r(j+k)}{|n-j||n-k|} .
$$

Следовательно,

$$
\left\|\widehat{T}^{2}\right\|^{2} \leqslant\left\|\widehat{V}: L^{\infty} \rightarrow L^{2}\right\|^{2} \cdot\left(\sum_{j \neq n}\left\|G^{12} e_{j}^{2}\right\|_{L^{\infty}}^{2}+\sum_{j \neq n}\left\|G^{21} e_{j}^{1}\right\|_{L^{\infty}}^{2}\right) .
$$

В силу (2.100) мы имеем $\|\widehat{V}\|_{\infty \rightarrow 2} \leqslant 2\|r\|$. С другой стороны, из леммы 6 вытекает, что

$$
\begin{aligned}
\sum_{j \neq n}\left\|G^{12} e_{j}^{2}\right\|_{\infty}^{2} & =\sum_{j \neq n}\left\|G^{21} e_{j}^{1}\right\|_{\infty}^{2}=\sum_{j \neq n}\left\|\sum_{k \neq n} \frac{|r(j+k)|}{|n-j \| n-k|} e_{k}^{1}\right\|_{\infty}^{2} \\
& \leqslant \sum_{j \neq n}\left(\sum_{k \neq n} \frac{r(j+k)}{|n-j||n-k|}\right)^{2} \leqslant C\left(\left(\mathscr{E}_{|n|}(r)\right)^{2}+\frac{\|r\|^{2}}{|n|}\right) .
\end{aligned}
$$

Но тогда мы имеем (2.103). Лемма 33 доказана. 
ЛЕмма 34. При принятых выше обозначениях если $|n|$ достаточно велико, mo

$$
A(r ; n) \leqslant C\|r\|\left(\mathscr{E}_{|n|}(r)+\frac{\|r\|}{\sqrt{|n|}}\right), \quad B(r ; n) \leqslant C\|r\|\left(\mathscr{E}_{|n|}(r)+\frac{\|r\|}{\sqrt{|n|}}\right),
$$

әде $C=C(\|r\|)$.

ДокАЗАТЕЛЬСтво. В силу (2.89) и (2.100)-(2.102), мы имеем:

$$
A(r ; n)=\left\langle\widehat{V} \widehat{D} Q_{n}^{0} \widehat{V} e_{n}^{1}, e_{n}^{1}\right\rangle+\left\langle\widehat{V} \widehat{D} \widehat{T}^{2}\left(1-\widehat{T}^{2}\right)^{-1} \widehat{V} e_{n}^{1}, e_{n}^{1}\right\rangle=\Sigma_{1}+\Sigma_{2},
$$

где

$$
\begin{aligned}
\Sigma_{1} & =\left\langle\widehat{V} \widehat{D} Q_{n}^{0} \widehat{V} e_{n}^{1}, e_{n}^{1}\right\rangle=\sum_{j \neq k} \frac{r(-n-j) r(n+j)}{|n-j|} \\
& \leqslant\left(\sum_{j \neq n} \frac{|r(n+j)|^{2}}{|n-j|^{2}}\right)^{1 / 2} \cdot\|r\| \leqslant\left(\mathscr{E}_{|n|}(r)+\frac{\|r\|}{\sqrt{|n|}}\right) \cdot\|r\|
\end{aligned}
$$

(по неравенству Коши и формуле (2.82));

$$
\begin{aligned}
\Sigma_{2} & =\left\langle\widehat{V} \widehat{D} \widehat{T}^{2}\left(1-\widehat{T}^{2}\right)^{-1} \widehat{V} e_{n}^{1}, e_{n}^{1}\right\rangle=\sum_{i, j \neq k} \frac{r(n+i) r(n+j)}{|n-i|} H_{i j}^{22} \\
& \leqslant\left(\|r\| \mathscr{E}_{|n|}(r)+\frac{\|r\|^{2}}{\sqrt{|n|}}\right) \cdot\|H\|_{\mathrm{HS}}
\end{aligned}
$$

(по лемме 31 , где $H=\widehat{T}^{2}\left(1-\widehat{T}^{2}\right)^{-1}$ ). По лемме $33,\left\|\widehat{T}^{2}\right\|_{\mathrm{HS}} \leqslant 1 / 2$ для достаточно больших $n$, скажем, $|n|>n_{0}$. Следовательно, оператор $H$ вполне определен для $|n|>n_{0}$, и при этом

$$
\|H\|_{\mathrm{HS}} \leqslant 2\left\|\widehat{T}^{2}\right\|_{\mathrm{HS}} \leqslant 1
$$

Соотношения (2.105)-(2.107) влекут за собой первое неравенство в (2.104). Мы опускаем доказательство второго неравенства в (2.104); оно проводится тем же способом. На самом деле выполнено намного более сильное утверждение - см. (2.113) в лемме 36. Лемма 34 доказана.

ПреДЛОЖЕНИЕ 35. Пусть $v(x)=\left(\begin{array}{cc}0 & P(x) \\ Q(x) & 0\end{array}\right)-$ периодический $L^{2}$-noтенциал Дирака на $[0, \pi]$ и

$$
P(x)=\sum_{m \in 2 \mathbb{Z}} p(m) e^{i m x}, \quad Q(x)=\sum_{m \in 2 \mathbb{Z}} q(m) e^{i m x} .
$$

Тогда при $r=(r(m)), r(m)=\max \{|p( \pm m)|, q( \pm m)\}$, мъь имеем:

$$
\left|\alpha_{n}(v ; z)\right|,\left|\beta_{n}^{ \pm}(v ; z)\right| \leqslant C\left(\mathscr{E}_{|n|}(r)+\frac{1}{\sqrt{|n|}}\right), \quad \text { если }|n| \geqslant n_{0}(v), \quad|z| \leqslant \frac{1}{2} .
$$


Более того,

$$
\left|\frac{\partial \alpha_{n}}{\partial z}(v ; z)\right|,\left|\frac{\partial \beta_{n}^{ \pm}}{\partial z}(v ; z)\right| \leqslant C\left(\mathscr{E}_{|n|}(r)+\frac{1}{\sqrt{|n|}}\right), \quad \text { еслu } \quad|n| \geqslant n_{0}(v), \quad|z| \leqslant \frac{1}{4},
$$

әде $C=C(\|r\|)$.

ДокАзАтЕльство. Ввиду (2.96), (2.98) и (2.99), неравенства (2.109) вытекают немедленно из леммы 34 .

Так как $\alpha$ и $\beta^{ \pm}$зависят аналитически от $z$ в круге $|z| \leqslant 1 / 2$, то неравенство Коши для их производных доказывает (2.110). Предложение 35 доказано.

ЛЕмма 36. Пусть $\omega_{1}=\left(\omega_{1}(m)\right)_{m \in 2 \mathbb{Z}}-$ медленно растущий вес такой, что

$$
\omega_{1}(m) \leqslant C_{1}|m|, \quad m \in 2 \mathbb{Z},
$$

$u r=(r(m))_{m \in 2 \mathbb{Z}} \in \ell^{2}\left(\omega_{1}, 2 \mathbb{Z}\right)$. Пусть $\bar{r}=(\bar{r}(m))_{m \in 2 \mathbb{Z}}$ определено формулой

$$
\bar{r}(m)=\omega_{1}(m) r(m), \quad m \in 2 \mathbb{Z} .
$$

Если $r \in \ell^{2}\left(\omega_{1}, 2 \mathbb{Z}\right)$, то для $N>n_{0}(\|r\|)$ мы имеем:

$$
\sum_{|n|>N}|B(r ; n)|^{2}\left|\omega_{1}(2 n)\right|^{2} \leqslant C\left(\frac{\left(\mathscr{E}_{N}(\bar{r})\right)^{4}}{\left(\omega_{1}(2 N)\right)^{2}}+\frac{\|\bar{r}\|^{4}}{N}\right)\|\bar{r}\|^{2},
$$

где $C=C\left(\omega_{1}\right)$.

ДокАзАтельство. Так как $\omega_{1}$ - медленно растущий вес, то существует такая постоянная $C_{0} \geqslant 1$, что

$$
\omega_{1}(2 m) \leqslant C_{0} \omega_{1}(m), \quad m \in 2 \mathbb{Z} .
$$

Легко видеть, что вес $C_{0} \omega_{1}(m)$ субмультипликативный.

Далее, мы имеем:

$B(r ; n)=\left\langle\widehat{V} \widehat{D} Q_{n}^{0} \widehat{V} \widehat{D} Q_{n}^{0} \widehat{V} e_{n}^{1}, e_{n}^{2}\right\rangle+\left\langle\widehat{V} \widehat{D} Q_{n}^{0} \widehat{T}^{3}\left(1-\widehat{T}^{2}\right)^{-1} \widehat{V} e_{n}^{1}, e_{n}^{2}\right\rangle=\Sigma_{3}(n)+\Sigma_{4}(n)$,

где

$$
\Sigma_{3}(n)=\left\langle\widehat{V} \widehat{D} Q_{n}^{0} \widehat{V} \widehat{D} Q_{n}^{0} \widehat{V} e_{n}^{1}, e_{n}^{2}\right\rangle=\sum_{i, j \neq n} \frac{r(n+i) r(-i-j) r(n+j)}{|n-i \| n-j|}
$$

и

$$
\Sigma_{4}(n)=\left\langle\widehat{V} \widehat{D} \widehat{T}^{3}\left(1-\widehat{T}^{2}\right)^{-1} \widehat{V} e_{n}^{1}, e_{n}^{2}\right\rangle=\sum_{i, j \neq n} \frac{r(n+i) r(n+j)}{|n-i|} H_{i j}^{21}(n),
$$

где $H=\widehat{T}^{3}\left(1-\widehat{T}^{2}\right)^{-1}$.

Так как

$$
|B(r ; n)|^{2} \leqslant 2\left(\Sigma_{3}(n)\right)^{2}+2\left(\Sigma_{4}(n)\right)^{2},
$$

то достаточно оценить $\sum_{|n|>N}\left(\omega_{1}(2 n)\right)^{2}\left(\Sigma_{3}(n)\right)^{2}$ и $\sum_{|n|>N}\left(\omega_{1}(2 n)\right)^{2}\left(\Sigma_{4}(n)\right)^{2}$. 
В силу (2.116), мы имеем:

$$
\left(\Sigma_{3}(n)\right)^{2} \leqslant 3\left(\sum_{|n-i|,|n-j| \leqslant|n|}\right)^{2}+3\left(\sum_{|n-i|>|n|, j \neq n}\right)^{2}+3\left(\sum_{|n-j|>|n|, i \neq n}\right)^{2} .
$$

Положим $\nu=n+i$ и $\mu=n+j$; тогда

$|n-i| \leqslant|n| \Rightarrow|\nu|=|2 n-(n-i)| \geqslant 2|n|-|n-i| \geqslant|n|,|n-j| \leqslant|n| \Rightarrow|\mu| \geqslant|n|$.

Следовательно, по неравенству Коши, $\sum_{|n|>N}\left(\omega_{1}(2 n)\right)^{2}\left(\sum_{|n-i|,|n-j| \leqslant|n|}\right)^{2}$ не превосходит

$$
\begin{aligned}
& \sum_{|n|>N}\left(\omega_{1}(2 n)\right)^{2} \sum_{i, j \neq n} \frac{1}{|n-i|^{2}|n-j|^{2}}\left(\sum_{|n-i|,|n-j| \leqslant|n|}|r(n+i)|^{2}|r(-i-j)|^{2}|r(n+j)|^{2}\right) \\
& \leqslant\left(\frac{\pi^{2}}{3}\right)^{2} \sum_{|n|>N}\left(\omega_{1}(2 n)\right)^{2} \sum_{|\nu|,|\mu| \geqslant|n|}|r(\nu)|^{2}|r(\mu)|^{2}|r(2 n-\nu-\mu)|^{2} .
\end{aligned}
$$

В силу (2.112) и (2.114), это выражение не превосходит

$$
\begin{aligned}
& \left(\frac{\pi^{2}}{3}\right)^{2} \sum_{|n|>N}\left(\omega_{1}(2 n)\right)^{2} \sum_{|\nu|,|\mu| \geqslant|n|} \frac{\left|C_{0} \bar{r}(\nu)\right|^{2}}{\left(\omega_{1}(2 \nu)\right)^{2}} \frac{\left|C_{0} \bar{r}(\mu)\right|^{2}}{\left(\omega_{1}(2 \mu)\right)^{2}}|r(2 n-\nu-\mu)|^{2} \\
& \quad \leqslant \frac{\pi^{4}}{9} \frac{C_{0}^{4}}{\left(\omega_{1}(2 N)\right)^{2}} \sum_{|\nu| \geqslant|n|}|\bar{r}(\nu)|^{2} \sum_{|\mu| \geqslant|n|}|\bar{r}(\mu)|^{2} \sum_{|n|>N}|r(2 n-\nu-\mu)|^{2} \\
& \leqslant \frac{\pi^{4}}{9} \frac{C_{0}^{4}}{\left(\omega_{1}(2 N)\right)^{2}}\left(\mathscr{E}_{N}(\bar{r})\right)^{4}\|r\|^{2} .
\end{aligned}
$$

Далее мы оцениваем сумму $\sum_{|n|>N}\left(\omega_{1}(2 n)\right)^{2}\left(\sum_{|n-i|>|n|, j \neq n}\right)^{2}$. Так как вес $C_{0} \omega_{1}$ субмультипликативный, то мы имеем:

$$
\omega_{1}(2 n) \leqslant C_{0}^{2} \omega_{1}(n+i) \omega_{1}(-i-j) \omega_{1}(n+j) .
$$

Следовательно,

$$
\begin{aligned}
& \sum_{|n|>N}\left(\omega_{1}(2 n)\right)^{2}\left(\sum_{|n-i|>|n|, j \neq n} \ldots\right)^{2} \\
& \quad \leqslant C_{0}^{4} \sum_{|n|>N}\left(\omega_{1}(2 n)\right)^{2}\left(\sum_{|n-i|>|n|, j \neq n} \frac{|\bar{r}(n+i) \bar{r}(-i-j) \bar{r}(n+j)|}{|n-i||n-j|}\right)^{2} .
\end{aligned}
$$

В силу (2.111) и неравенства Коши, последняя сумма не превосходит

$$
\begin{gathered}
C_{0}^{4} \sum_{|n|>N}\left(\sum_{i, j \neq n} \frac{|\bar{r}(n+i)|^{2}}{n^{2}|n-j|^{2}}\right) \cdot\left(\sum_{i, j}|\bar{r}(-i-j)|^{2}|\bar{r}(n+j)|^{2}\right) \\
\leqslant C_{0}^{4} \sum_{|n|>N} \frac{1}{|n|^{2}} \sum_{j \neq n} \frac{1}{|n-j|^{2}} \cdot\|\bar{r}\|^{6} \leqslant \frac{\widetilde{C}}{N}\|\bar{r}\|^{6}
\end{gathered}
$$


где $\widetilde{C}=\widetilde{C}\left(\omega_{1}\right)$.

По соображениям симметрии такая же оценка выполнена и для суммы $\sum_{|n|>N}\left(\omega_{1}(2 n)\right)^{2}\left(\sum_{|n-j|>|n|, i \neq n}\right)^{2}$. Таким образом, мы имеем:

$$
\sum_{|n|>N}\left(\omega_{1}(2 n)\right)^{2}\left(\Sigma_{3}(n)\right)^{2} \leqslant C\left(\frac{\left(\mathscr{E}_{N}(\bar{r})\right)^{4}}{\left(\omega_{1}(2 N)\right)^{2}}+\frac{\|\bar{r}\|^{4}}{N}\right)\|\bar{r}\|^{2},
$$

где $C=C\left(\omega_{1}\right)$.

Мы оцениваем сумму $\sum_{|n|>N}\left(\omega_{1}(2 n)\right)^{2}\left(\Sigma_{4}(n)\right)^{2}$ по лемме 32 , применяя ее к выражению для $\Sigma_{4}(n)$, данному в $(2.117)$, где $H=\widehat{T}^{3}\left(1-\widehat{T}^{2}\right)^{-1}$.

По лемме $33,\left\|\widehat{T}^{2}\right\|_{\text {нS }}<1 / 2$ для достаточно больших $n$, скажем, $|n|>n_{0}(\|r\|)$. С другой стороны, мы имеем:

$$
\|\widehat{T}\|_{\mathrm{HS}}^{2} \leqslant 2 \sum_{i, j \neq n} \frac{|r(i+j)|^{2}}{|n-j|^{2}} \leqslant 2 \sum_{j \neq n} \frac{1}{|n-j|^{2}} \sum_{i}|r(i+j)|^{2} \leqslant \frac{2 \pi^{2}}{3}\|r\|^{2} .
$$

Следовательно, оператор $H(n)$ вполне определен, если $|n|>n_{0}$, и при этом

$$
\|H(n)\|_{\mathrm{HS}} \leqslant\|\widehat{T}\|_{\mathrm{HS}} \leqslant 3\|r\| .
$$

Таким образом, лемма 32 влечет за собой неравенство

$$
\sum_{|n|>N}\left(\omega_{1}(2 n)\right)^{2}\left(\Sigma_{4}(n)\right)^{2} \leqslant C\left(\frac{\left(\mathscr{E}_{N}(\bar{r})\right)^{4}}{\left(\omega_{1}(2 N)\right)^{2}}+\frac{\|\bar{r}\|^{4}}{N}\right)\|\bar{r}\|^{2},
$$

где $C=C\left(\omega_{1}\right)$.

Наконец, из (2.118), (2.120) и (2.122) следует, что выполнено (2.113). Это завершает доказательство леммы 36.

Если $\omega=(\omega(m))_{m \in 2 \mathbb{Z}}$ - субмультипликативный вес, то мы положим $\omega r=$ $(\omega(m) r(m))_{m \in 2 \mathbb{Z}}$.

ЛЕмма 37. При принятых выше обозначениях если $\omega$ - субмультипликативный вес на $2 \mathbb{Z}$, mо

$$
B(r ; n) \omega(2 n) \leqslant B(\omega r ; n) .
$$

ДокАзАТЕЛьство. Ввиду (2.89), достаточно показать, что

$$
B_{2 \nu}(r ; n) \omega(2 n) \leqslant B_{2 \nu}(\omega r ; n), \quad \nu \in \mathbb{N} .
$$

Так как $\omega$ - субмультипликативный вес, то для каждого $2 \nu$-набора индексов $j_{1}, \ldots, j_{2 \nu}$ мы имеем:

$\omega(2 n)=\omega(-2 n) \leqslant \omega\left(-n-j_{1}\right) \omega\left(j_{1}+j_{2}\right) \omega\left(-j_{2}-j_{3}\right) \cdots \omega\left(j_{2 \nu-1}+j_{2 \nu}\right) \omega\left(-j_{2 \nu}-n\right)$.

Поэтому

$$
\begin{aligned}
& r\left(-n-j_{1}\right) r\left(j_{1}+j_{2}\right) \cdots r\left(-j_{2 \nu}-n\right) \omega(2 n) \\
& \quad \leqslant\left[r\left(-n-j_{1}\right) \omega\left(-n-j_{1}\right)\right]\left[r\left(j_{1}+j_{2}\right) \omega\left(j_{1}+j_{2}\right)\right] \cdots\left[r\left(-j_{2 \nu}-n\right) \omega\left(-j_{2 \nu}-n\right)\right],
\end{aligned}
$$

откуда вытекает соотношение (2.123). Лемма 37 доказана. 
ЛЕмма 38. Пусть $\omega=(\omega(m))_{m \in 2 \mathbb{Z}}-$ субмультипликативный вес $и ~ r=$ $(r(m))_{m \in 2 \mathbb{Z}} \in \ell^{2}(\omega, 2 \mathbb{Z})$. Тогда для достаточно больших $n_{0}$ мы имеем:

$$
\sum_{|n|>n_{0}}|B(r ; n) \omega(2 n)|^{2}<\infty
$$

ДокАзАТЕЛьство. В силу леммы 37,

$$
B(r ; n) \omega(2 n) \leqslant B(\omega r ; n),
$$

где $\omega r \in \ell^{2}(2 \mathbb{Z})$. Поэтому достаточно показать, что если $r \in \ell^{2}(2 \mathbb{Z})$, то

$$
\sum_{|n|>n_{0}}|B(r ; n)|^{2}<\infty
$$

Это вытекает из леммы 36 , где выбран вес $\omega_{1}(m) \equiv 1$. Лемма 38 доказана.

ПРЕДЛОЖЕНИЕ 39. Пусть $\Omega=(\Omega(k))_{k \in \mathbb{Z}}-$ вес вида

$$
\Omega(k)=\Omega_{1}(k) \Omega_{2}(k), \quad k \in \mathbb{Z},
$$

где $\Omega_{1}$ - медленно растущий вес такой, что

$$
\Omega_{1}(k) \leqslant C_{1}|k|,
$$

a $\Omega_{2}$ - субмультипликативный вес. Если потенциал Дирака

$$
v(x)=\left(\begin{array}{cc}
0 & P(x) \\
Q(x) & 0
\end{array}\right), \quad P(x)=\sum_{m \in \mathbb{Z}} P_{m} e^{i m x}, \quad Q(x)=\sum_{m \in \mathbb{Z}} Q_{m} e^{i m x},
$$

лежит в соболевском пространстве $H_{D}(\Omega)$, m.е.

$$
\|v\|_{\Omega}^{2}=\sum_{k \in \mathbb{Z}}\left[\left|P_{k}\right|^{2}+\left|Q_{k}\right|^{2}\right](\Omega(k))^{2}<\infty,
$$

то для $N>n_{0}(\|v\|) u|z|<1 / 2$ мы имеем:

$$
\sum_{|n|>N}\left(\left|\beta_{n}^{-}(z)-P_{-n}\right|^{2}+\left|\beta_{n}^{+}(z)-Q_{n}\right|^{2}\right)(\Omega(n))^{2} \leqslant C\left(\frac{1}{\left(\Omega_{1}(N)\right)^{2}}+\frac{1}{N}\right)\|v\|_{\Omega}^{6},
$$

әде $C=C\left(\Omega_{1}\right)$.

ДоказАтельство. Рассмотрим вес $\omega=(\omega(m))_{m \in 2 \mathbb{Z}}$ где $\omega(m)=\Omega(m / 2)$. Конечно, $\omega(m)=\omega_{1}(m) \omega_{2}(m)$, где $\omega_{1}$ - медленно растущий вес такой, что $\omega_{1}(m)=\Omega_{1}(m / 2)$, а $\omega_{2}-$ субмультипликативный вес такой, что $\omega_{2}(m)=$ $\Omega_{2}(m / 2)$.

В силу (2.98) и (2.99), мы имеем:

$$
\left|\beta_{n}^{-}(z)-p(-2 n)\right|+\left|\beta_{n}^{+}(z)-q(2 n)\right| \leqslant 2 B(r ; n),
$$

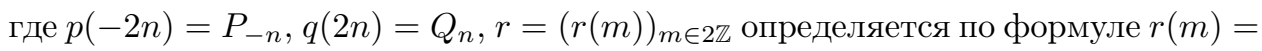
$\max \left(\left|P_{ \pm m}\right|,\left|Q_{ \pm m / 2}\right|\right)$, и, следовательно,

$$
\frac{1}{2}\|r\|_{\omega} \leqslant\|v\|_{\Omega} \leqslant 2\|r\|_{\omega} .
$$


Таким образом, достаточно оценить $\sum_{|n|>N}|B(r ; n)|^{2}(\omega(2 n))^{2}$.

По лемме 37 мы имеем:

$$
B(r ; n) \omega_{2}(2 n) \leqslant B\left(\omega_{2} r ; n\right),
$$

и, следовательно,

$$
\sum_{|n|>N}|B(r ; n)|^{2}(\omega(2 n))^{2} \leqslant \sum_{|n|>N}\left|B\left(\omega_{2} r ; n\right)\right|^{2}\left(\omega_{1}(2 n)\right)^{2} .
$$

По неравенству (2.113) в лемме 36, последняя сумма не превосходит

$$
C\left(\frac{1}{\left(\Omega_{1}(N)\right)^{2}}+\frac{1}{N}\right)\|v\|_{\Omega}^{6},
$$

где $C=C\left(\omega_{1}\right)$. Это завершает доказательство предложения 39 .

2.6. Оценки $\left|\gamma_{n}\right|$ сверху. В этом пункте мы рассматриваем операторы Хилла-Шрёдингера и Дирака одновременно. В силу локализационных теорем 9 и 17, для достаточно больших $|n|$ оператор $L=L^{0}+V$ имеет два (периодических, если $n$ четно, и антипериодических, если $n$ нечетно) собственных значения $\lambda_{n}^{-}$и $\lambda_{n}^{+}$таких, что $\left|\lambda_{n}^{ \pm}-\lambda_{n}^{0}\right| \leqslant r$, где $\lambda_{n}^{0}=n^{2}, r=10(1+\|V\|)$ в случае Хилла-Шрёдингера и $\lambda_{n}^{0}=n, r=1 / 4$ в случае Дирака. Теперь мы оценим $\left|\gamma_{n}\right|=\left|\lambda_{n}^{+}-\lambda_{n}^{-}\right|$сверху.

ЛЕмма 40. В обоих случаях при достаточно больших $|n|$ мы имеем:

$$
\left|\gamma_{n}\right|=\left|\lambda_{n}^{+}-\lambda_{n}^{-}\right| \leqslant\left(1+\delta_{n}\right)\left(\left|\beta_{n}^{-}\left(z_{n}^{*}\right)\right|+\left|\beta_{n}^{+}\left(z_{n}^{*}\right)\right|\right), \quad z_{n}^{*}=\frac{\lambda_{n}^{+}+\lambda_{n}^{-}}{2}-\lambda_{n}^{0},
$$

где $\delta_{n} \rightarrow 0$ nрu $|n| \rightarrow \infty$.

ДокАЗАТЕЛЬСтво. По лемме 21 числа

$$
z_{n}^{ \pm}=\lambda_{n}^{ \pm}-\lambda_{n}^{0}
$$

являются собственными значениями оператора $S$. Поэтому $z_{n}^{+}$и $z_{n}^{-}-$корни основного уравнения (2.23), или (2.58). Перепишем (2.23), или (2.58), в виде

$$
\left(\zeta_{n}(z)\right)^{2}=\beta_{n}^{-}(z) \beta_{n}^{+}(z),
$$

где

$$
\zeta_{n}(z)=z-\alpha_{n}(v ; z)
$$

По локализационным теоремам (теорема 9 для случая Хилла-Шрёдингера и теорема 17 для случая Дирака) и по лемме 29 в первом случае и по предложению 35 во втором, мы имеем:

$$
\sup _{\left[z_{n}^{-}, z_{n}^{+}\right]}\left|\frac{\partial \alpha_{n}}{\partial z}\right| \leqslant \varepsilon_{n}, \quad \sup _{\left[z_{n}^{-}, z_{n}^{+}\right]}\left|\frac{\partial \beta_{n}^{ \pm}}{\partial z}\right| \leqslant \varepsilon_{n}, \quad \varepsilon_{n} \downarrow 0,
$$

где $\left[z_{n}^{-}, z_{n}^{+}\right]$обозначает отрезок с концевыми точками $z_{n}^{-}$и $z_{n}^{+}$. 
Следовательно, ввиду (2.132), мы имеем:

$$
\begin{aligned}
\left|z_{n}^{+}-z_{n}^{-}\right| & \leqslant\left|\zeta_{n}\left(z_{n}^{+}\right)-\zeta_{n}\left(z_{n}^{-}\right)\right|+\left|\alpha\left(z_{n}^{+}\right)-\alpha\left(z_{n}^{-}\right)\right| \\
& \leqslant\left|\zeta_{n}\left(z_{n}^{+}\right)-\zeta_{n}\left(z_{n}^{-}\right)\right|+\varepsilon_{n}\left|z_{n}^{+}-z_{n}^{-}\right|
\end{aligned}
$$

где $\varepsilon_{n} \rightarrow 0$, когда $n \rightarrow \infty$. Таким образом, для больших $n$ мы имеем:

$$
\left(1-\varepsilon_{n}\right)\left|z_{n}^{+}-z_{n}^{-}\right| \leqslant\left|\zeta_{n}\left(z_{n}^{+}\right)-\zeta_{n}\left(z_{n}^{-}\right)\right| .
$$

Так как

$$
\zeta_{n}\left(z_{n}^{+}\right)-\zeta_{n}\left(z_{n}^{-}\right)=\int_{z_{n}^{-}}^{z_{n}^{+}}\left(1-\frac{\partial \alpha_{n}}{\partial z}\right) d z,
$$

то, в силу (2.133), мы получаем, что

$$
\left|\zeta_{n}\left(z_{n}^{+}\right)-\zeta_{n}\left(z_{n}^{-}\right)\right| \leqslant\left(1+\varepsilon_{n}\right)\left|z_{n}^{+}-z_{n}^{-}\right| .
$$

Таким образом, мы имеем следующие двусторонние оценки:

$$
\left(1-\varepsilon_{n}\right)\left|z_{n}^{+}-z_{n}^{-}\right| \leqslant\left|\zeta_{n}\left(z_{n}^{+}\right)-\zeta_{n}\left(z_{n}^{-}\right)\right| \leqslant\left(1+\varepsilon_{n}\right)\left|z_{n}^{+}-z_{n}^{-}\right| .
$$

С другой стороны, в силу (2.131), мы имеем:

$$
\left|\zeta_{n}(z)\right| \leqslant \frac{1}{2}\left(\left|\beta_{n}^{-}(z)\right|+\left|\beta_{n}^{+}(z)\right|\right) \quad \text { для } \quad z=z_{n}^{ \pm} .
$$

Таким образом, в силу (2.134),

$$
\left(1-\varepsilon_{n}\right)\left|z_{n}^{+}-z_{n}^{-}\right| \leqslant \frac{1}{2}\left(\left|\beta_{n}^{-}\left(z_{n}^{-}\right)\right|+\left|\beta_{n}^{-}\left(z_{n}^{+}\right)\right|+\left|\beta_{n}^{+}\left(z_{n}^{-}\right)\right|+\left|\beta_{n}^{+}\left(z_{n}^{+}\right)\right|\right) .
$$

По оценкам для $\partial \beta / \partial z$, данным в лемме 29, в случае Хилла-Шрёдингера и по предложению 35 в случае Дирака, мы имеем:

$$
\left|\beta_{n}^{ \pm}\left(z_{n}^{ \pm}\right)-\beta_{n}^{ \pm}\left(z_{n}^{*}\right)\right| \leqslant \varepsilon_{n}\left|z_{n}^{+}-z_{n}^{-}\right| / 2,
$$

где можно предполагать, что $\varepsilon_{n}$ такое же, как в (2.133). Таким образом, мы получаем:

$$
\left(1-3 \varepsilon_{n}\right)\left|z_{n}^{+}-z_{n}^{-}\right| \leqslant\left|\beta_{n}^{+}\left(z_{n}^{*}\right)\right|+\left|\beta_{n}^{-}\left(z_{n}^{*}\right)\right|,
$$

откуда, в силу (2.130), вытекает (2.129). Лемма 40 доказана.

Теорема 41. Пусть $L=L^{0}+v(x), L^{0}=-d^{2} / d x^{2},-$ это периодический оператор Хилла-Шрёдингера на отрезке $I=[0, \pi]$ c $L^{2}(I)$-потенииалом $v(x)=$ $\sum_{k \in \mathbb{Z}} v_{k} e^{2 k i x}$. Тогда для $n>n_{0}(\|v\|)$ оператор $L$ имеет в круге с иентром $n^{2} u$ радиусом $r=r(\|v\|)$ в точности два (с учетом их алгебраической кратности) периодических (если п четно) или антипериодических (если $n$ нечетно) собственных значения $\lambda_{n}^{+}$и $\lambda_{n}^{-}$. Более того, для любого субмультипликативного веса $\Omega=(\Omega(m))_{m \in \mathbb{Z}}$

$$
\sum_{k \in \mathbb{Z}}\left|v_{k}\right|^{2}(\Omega(k))^{2}<\infty \Rightarrow \sum_{n>n_{0}(v)}\left|\gamma_{n}\right|^{2}(\Omega(n))^{2}<\infty,
$$

əде $\gamma_{n}=\lambda_{n}^{+}-\lambda_{n}^{-}$. 
ДокАЗАТЕЛЬСтво. По лемме 40 для достаточно больших $n$ мы имеем:

$$
\left|\gamma_{n}\right| \leqslant 2\left(\left|\beta_{n}^{-}\left(z_{n}^{*}\right)\right|+\left|\beta_{n}^{+}\left(z_{n}^{*}\right)\right|\right)
$$

где $z_{n}^{*}=\left(\lambda_{n}^{-}+\lambda_{n}^{+}\right) / 2-n^{2}$. Таким образом, в силу части (b) предложения 28

$$
\begin{aligned}
\left|\gamma_{n}\right| \Omega(n) & \leqslant 2\left(\left|v_{-n}\right|+\left|\widetilde{\beta}_{n}^{-}\left(v ; z_{n}^{*}\right)\right|+\left|v_{n}\right|+\left|\widetilde{\beta}_{n}^{+}\left(v ; z_{n}^{*}\right)\right|\right) \Omega(n) \\
& \leqslant 2\left|v_{-n}\right| \Omega(n)+2\left|v_{n}\right| \Omega(n)+\frac{16}{n}\|v\|_{H(\Omega)}^{2} .
\end{aligned}
$$

Таким образом, мы получаем:

$$
\sum_{n>N}\left|\gamma_{n}\right|^{2}(\Omega(n))^{2} \leqslant 12 \sum_{|n|>N}\left|v_{n}\right|^{2}(\Omega(n))^{2}+\frac{768}{N}\|v\|_{H(\Omega)}^{4},
$$

что доказывает соотношение (2.135). Теорема 41 доказана.

Аналогичное утверждение выполнено для операторов Дирака.

TEOPEMA 42. Если

$$
L=L^{0}+v(x), \quad L^{0}=i\left(\begin{array}{cc}
1 & 0 \\
0 & -1
\end{array}\right) \frac{d}{d x}
$$

- оператор Дирака на отрезке $I=[0, \pi] c\left(L^{2}(I)\right)^{2}$-потенииалом

$$
v(x)=\left(\begin{array}{cc}
0 & P(x) \\
Q(x) & 0
\end{array}\right), \quad P(x)=\sum_{m \in \mathbb{Z}} P_{m} e^{i m x}, \quad Q(x)=\sum_{m \in \mathbb{Z}} Q_{m} e^{i m x},
$$

то для $n \in \mathbb{Z},|n|>n_{0}(v)$, оператор $L$ имеет в круге с иентром $n$ и радиусом $r=1 / 4$ в точности два (с учетом их кратности) периодических (если $n$ четно) или антипериодических (если $n$ нечетно) собственных значения $\lambda_{n}^{+}$ $u \lambda_{n}^{-}$. Более того, для любого субмультипликативного веса $\Omega=(\Omega(m))_{m \in \mathbb{Z}}$

$$
\sum_{m \in \mathbb{Z}}\left(\left|P_{m}\right|^{2}+\left|Q_{m}\right|^{2}\right)(\Omega(m))^{2}<\infty \Rightarrow \sum_{|n|>n_{0}(v)}\left|\gamma_{n}\right|^{2}(\Omega(n))^{2}<\infty,
$$

əде $\gamma_{n}=\lambda_{n}^{+}-\lambda_{n}^{-}$.

ДокАЗАТЕЛЬСтво. По лемме 40, если $|n|$ достаточно велико, то

$$
\left|\gamma_{n}\right| \leqslant 2\left(\left|\beta_{n}^{-}\left(z_{n}^{*}\right)\right|+\left|\beta_{n}^{+}\left(z_{n}^{*}\right)\right|\right)
$$

где $z_{n}^{*}=\left(\lambda_{n}^{-}+\lambda_{n}^{+}\right) / 2-n$. Следовательно, из (2.98) и (2.99) вытекает, что

$$
\left|\gamma_{n}\right| \leqslant 2\left(\left|P_{-n}\right|+\left|Q_{n}\right|+2 B(r ; n)\right)
$$

где $r=(r(m))_{m \in 2 \mathbb{Z}}$ определено соотношением $r(m)=\max \left(\left|P_{ \pm m / 2}\right|,\left|Q_{ \pm m / 2}\right|\right)$.

Чтобы применить лемму 38 , мы рассмотрим вес $\omega=(\omega(m))_{m \in 2 \mathbb{Z}}$, заданный как $\omega(m)=\Omega(m / 2)$. По лемме 38 мы имеем:

$$
\sum_{|n|>n_{0}}|B(r ; n) \Omega(n)|^{2}=\sum_{|n|>n_{0}}|B(r ; n) \omega(2 n)|^{2}<\infty .
$$


Следовательно,

$$
\begin{aligned}
\sum_{|n|>n_{0}}\left|\gamma_{n}\right|^{2}(\Omega(n))^{2} \leqslant & C\left(\sum_{|n|>n_{0}}\left(\left|P_{-n}\right| \Omega(n)\right)^{2}\right. \\
& \left.+\sum_{|n|>n_{0}}\left(\left|Q_{n}\right| \Omega(n)\right)^{2}+\sum_{|n|>n_{0}}(|B(r ; n)| \Omega(n))^{2}\right)<\infty
\end{aligned}
$$

Это заканчивает доказательство теоремы 42 .

2.7. Примечания. Редукционная схема в п. 2.1 по существу есть один из вариантов метода Ляпунова-Шмидта, широко используемого в решении операторных и нелинейных уравнений, - см., например, [50] и библиографию в этой книге. Мы конструируем лемму 21 специально для наших целей анализа пар собственных значений оператора $L$, определяющих спектральные лакуны. Простая лемма 24(а), подмеченная в [36], [37], оказалась критической, посколько теперь лакуны не зависят от величины самих диагональных элементов $S^{11}$ и $S^{22}$. В случае Хилла-Шрёдингера подход пп. 2.2, 2.3 был развит в [36], [37] и далее в [38], [39], [29], а в случае Дирака - в [19]. Соответственно, теорема 41 была впервые доказана в [37], а теорема 42 - в [18], [19]. Частные случаи теоремы 42 при специальных ограничениях на веса $\Omega$ можно встретить в [16], [17], [51].

\section{3. Обратные оценки в самосопряженном случае}

Оператор Шрёдингера (1.1) - самосопряженный тогда и только тогда, когда его потенциал $v(x)$ вещественнозначный. Оператор Дирака (1.9) с потенциалом $v(x)=\left(\begin{array}{cc}0 & P(x) \\ Q(x) & 0\end{array}\right)$ - самосопряженный тогда и только тогда, когда $Q(x)=\overline{P(x)}$. В этом разделе мы изучаем, как гладкость потенциала $v(x)$ самосопряженного оператора Шрёдингера (или Дирака) зависит от скорости убывания последовательности лакун $\gamma=\left(\gamma_{n}\right)$.

3.1. Веса. Последовательность положительных чисел $\Omega=(\Omega(n))_{n \in \mathbb{Z}}$ назовем весом, или весовой последовательностью. В этой статье мы рассматриваем только четные монотонно растущие веса, т.е.

$$
\Omega(-n)=\Omega(n), \quad n \in \mathbb{Z},
$$

и

$$
\Omega(0)=1, \quad \Omega(n) \leqslant \Omega(n+1) \quad \text { для } n \geqslant 0 .
$$

Каждый вес $\Omega$ порождает соответствующее весовое $\ell^{2}$-пространство

$$
\ell^{2}(\Omega, \mathbb{Z})=\left\{x=\left(x_{n}\right)_{n \in \mathbb{Z}}:\|x\|^{2}=\sum_{n \in \mathbb{Z}}\left|x_{n}\right|^{2}(\Omega(n))^{2}<\infty\right\} .
$$

Мы рассматриваем также соответствующее соболевское пространство шрёдингеровских потенциалов

$$
H(\Omega)=\left\{v(x)=\sum_{k \in \mathbb{Z}} v_{k} e^{2 i k x}:\|v\|_{\Omega}^{2}=\sum\left|v_{k}\right|^{2}(\Omega(k))^{2}<\infty\right\} .
$$


В случае Дирака потенциалы имеют вид $v(x)=\left(\begin{array}{cc}0 & P(x) \\ Q(x) & 0\end{array}\right)$, где

$$
P(x)=\sum_{k \in \mathbb{Z}} P_{k} e^{2 i k x}, \quad Q(x)=\sum_{k \in \mathbb{Z}} Q_{k} e^{2 i k x} .
$$

Соответствующее весовое соболевское пространство потенциалов Дирака есть

$$
H_{D}(\Omega)=\left\{v:\|v\|_{\Omega}^{2}=\sum_{k \in \mathbb{Z}}\left(\left|P_{k}\right|^{2}+\left|Q_{k}\right|^{2}\right)(\Omega(k))^{2}<\infty\right\} .
$$

Мы говорим, что два веса $\Omega_{1}$ и $\Omega_{2}$ эквивалентны, если

$$
\exists C \geqslant 1: \quad C^{-1} \Omega_{1}(n) \leqslant \Omega_{2}(n) \leqslant C \Omega_{1}(n), \quad n \in \mathbb{Z} .
$$

Очевидно, эквивалентные веса приводят к эквивалентным нормам, так что они порождают одно и то же весовое $\ell^{2}$-пространство и одно и то же соболевское пространство.

Вес $\Omega$ называется субмультипликативным, если

$$
\Omega(n+m) \leqslant \Omega(n) \Omega(m), \quad n, m \in \mathbb{Z} .
$$

Конечно, если $\Omega_{1}$ и $\Omega_{2}$ - эквивалентные веса, то будь один из них субмультипликативным, другой удовлетворяет неравенству

$$
\Omega(n+m) \leqslant C \Omega(n) \Omega(m), \quad n, m \in \mathbb{Z},
$$

с некоторой постоянной $C>0$. Очевидно, если для $\Omega$ выполнено (3.5), то для $\widetilde{\Omega}=C \Omega$ выполнено (3.4). Более того, легко видеть, что если (3.5) выполняется для $n, m$ таких, что $|n|,|m| \geqslant n_{0}$, то оно выполнено для всех $n, m \in \mathbb{Z}$, хотя, быть может, с другой постоянной $C$.

Напомним, что вес $\Omega$ называется медленно растущим, если

$$
M:=\sup _{n} \frac{\Omega(2 n)}{\Omega(n)}<\infty .
$$

Легко видеть, что всякий медленно растущий вес эквивалентен субмультипликативному весу. Более того, из (3.6) вытекает:

$$
\exists a \geqslant 0, C>0: \quad \Omega(n) \leqslant C|n|^{a}, \quad \text { если }|n| \geqslant 1 .
$$

Действительно, в силу (3.6) мы имеем:

$$
\Omega\left(2^{k}\right) \leqslant \Omega(1) M^{k}=\Omega(1)\left(2^{k}\right)^{a}, \quad a=\log _{2}(M) .
$$

Теперь (так как $\Omega$ монотонно возрастает) (3.7) влечет за собой следующее: если $2^{k} \leqslant n<2^{k+1}$, то

$$
\Omega(n) \leqslant \Omega\left(2^{k+1}\right) \leqslant M \Omega\left(2^{k}\right) \leqslant M \Omega(1)\left(2^{k}\right)^{a} \leqslant M \Omega(1) n^{a} .
$$


ЛЕмма 43. Если $\Omega=(\Omega(n))_{n \in \mathbb{Z}}-$ медленно растущий вес, то существует эквивалентный медленно растущии вес $\Omega^{*}$ такой, что

$$
\frac{\log \Omega^{*}(n)}{n} \searrow 0, \quad \text { когда } \quad n \rightarrow \infty .
$$

ДокАЗАТЕЛЬство. Пусть $h(n)=\log (\Omega(n))$. Поскольку $\Omega$ - медленно растущий вес, то

$$
\exists c>0: \quad h(2 n) \leqslant h(n)+c .
$$

Без потери общности мы можем предполагать, что $h(1)>c$ (иначе можно заменить $\Omega$ на эквивалентный вес). Тогда из (3.9) вытекает, что $h(2 n) \leqslant 2 h(n)$ для всех $n \in \mathbb{N}$, и поэтому мы имеем:

$$
\frac{h\left(2^{n}\right)}{2^{n}} \geqslant \frac{h\left(2^{n+1}\right)}{2^{n+1}} \quad \forall n \in \mathbb{N} .
$$

Положим $\Omega^{*}(m)=\exp \left(h^{*}(m)\right)$, где $h^{*}(1)=h(1)$ и

$$
h^{*}\left(2^{n}+k\right)=h\left(2^{n}\right)+\frac{k}{2^{n}}\left(h\left(2^{n+1}\right)-h\left(2^{n}\right)\right), \quad 0 \leqslant k \leqslant 2^{n}, \quad n \in \mathbb{N} .
$$

Тогда $h^{*}\left(2^{n}\right)=h\left(2^{n}\right)$ для $n \in \mathbb{N}$, и поэтому легко видеть, что $\Omega$ и $\Omega^{*}$ - эквивалентные веса. Более того, из (3.10) вытекает:

$$
\frac{h^{*}\left(2^{n}\right)}{2^{n}} \geqslant \frac{h^{*}\left(2^{n+1}\right)}{2^{n+1}} \quad \forall n \in \mathbb{N} .
$$

Теперь легко видеть, что (3.8) выполнено - например, по геометрическим соображениям, поскольку $h^{*}$ линейно на каждом отрезке вида $\left[2^{n}, 2^{n+1}\right]$ и выполняется соотношение (3.12). Лемма 43 доказана.

Если $\sup _{n} \Omega(2 n) / \Omega(n)=\infty$ (так что, конечно, $\Omega$ не есть медленно растущий вес), то $\Omega$ называется быстро растущим весом. Быстро растущий субмультипликативный вес $\Omega$ растет не быстрее, чем экспоненциально, поскольку

$$
\Omega(n) \leqslant(\Omega(1))^{|n|}=e^{a|n|}, \quad a=\log \Omega(1) .
$$

Любой вес можно записать в виде

$$
\Omega(n)=\exp (h(|n|)), \quad \text { где } \quad h(|n|)=\log \Omega(|n|), \quad h(0)=0 .
$$

Поэтому свойства веса $\Omega$ можно охарактеризовать в терминах функции $h$. Например, вес $\Omega$ субмультипликативен тогда и только тогда, когда функция $h$ субаддитивна, т.е.

$$
h(n+m) \leqslant h(n)+h(m) \quad \forall n, m \in \mathbb{N} .
$$

Хорошо известно (см., например, [52]), что если $(h(n))$ - субаддитивная последовательность, то предел

$$
\ell=\lim _{n \rightarrow \infty} \frac{h(n)}{n}
$$

существует. Субмультипликативный вес $\Omega$ вида (3.13) назовем субэкспоненииалъным, если $\ell=0$, и экспоненциальным, если $\ell>0$. 
Лемма 44. Если $\Omega$ - вес вида (3.13) такой, что соответствующая последовательность $(h(n))_{n=0}^{\infty}$ вогнута, т.е. удовлетворяет соотношению

$$
h(n+2)-h(n+1) \leqslant h(n+1)-h(n) \quad \text { для } n \geqslant 0,
$$

то $\Omega$ - субмультипликативный вес.

ДокАЗАТЕЛЬСтво. Ввиду (3.1) и (3.2), достаточно проверить (3.4) при $m, n>0$. Зафиксируем $m, n \in \mathbb{N}$. Тогда, в силу (3.16), мы имеем:

$$
h(n+m)-h(n)=\sum_{i=1}^{m}[h(n+i)-h(n+i-1)] \leqslant \sum_{i=1}^{m}[h(i)-h(i-1)]=h(m) .
$$

Таким образом, (3.14) выполнено, т.е. вес $\Omega(n)=\exp (h(|n|))$ субмультипликативен. Лемма 44 доказана.

Заметим, что если (3.16) выполнено лишь для $n \geqslant n_{0}>0$, то $\Omega$ эквивалентен субмультипликативному весу. Действительно, мы можем определить новую функцию $\widetilde{h}$ так, чтобы она удовлетворяла соотношениям (3.16) и $\widetilde{h}(n)=h(n)+C$ для $n \geqslant n_{0}$, где $C \geqslant 0$ - некоторая постоянная. Тогда (3.14) будет выполнено для новой функции. Соответствующий вес $\widetilde{\Omega}$ эквивалентен весу $\Omega$, и тогда, по лемме 44 , вес $\widetilde{\Omega}$ - субмультипликативный. Поэтому верно следующее утверждение.

ЗАмЕчАНИЕ 45. Если $\Omega$ - такой вес, что $\Omega(n)=h(|n|)$ для $|n| \geqslant n_{0}$, где $h:\left[n_{0}, \infty\right) \rightarrow(0, \infty)$ - возрастающая вогнутая функция, то $\Omega$ эквивалентен субмультипликативному весу.

Характерные и важные в анализе примеры субмультипликативных весов это соболевские веса

$$
\Omega_{a}(n)=|2 n|^{a}, \quad n \neq 0, \quad a>0,
$$

и жевреевские веса

$$
\Omega_{a, b}(n)=\exp \left(a|n|^{b}\right), \quad a>0, \quad b \in(0,1) .
$$

Соответствующие функции $h$ вогнуты. Конечно, можно дать много других примеров, используя замечание 45. Упомянем, однако, что существуют субмультипликативные веса вида $\Omega(n)=\exp (h(|n|))$, где функция $h$ - не вогнутая, т.е. она не удовлетворяет неравенству (3.16). Мы не будем давать сейчас таких (контр)примеров. Мы склонны думать, что класс субмультипликативных весов слишком широк; мы выделяем его достаточно разумный подкласс, рассматривая веса вида $\Omega(n)=\exp (h(|n|))$, где $h$ вогнута (т.е. удовлетворяет соотношению (3.16)).

Следующее утверждение дает нам более слабое условие, обеспечивающее субмультипликативность веса.

Лемма 46. Пусть вес $\Omega$ таков, что функиия $h$ в его представлении (3.13) удовлетворяет соотношению

$$
\frac{h(n)}{n} \geqslant \frac{h(n+1)}{n+1}, \quad n \in \mathbb{N} .
$$

Тогда $\Omega$ - субмультипликативнъй вес. 
Доказательство. Действительно, из (3.19) вытекает для всех $n, m \in \mathbb{N}$, что

$$
\frac{n}{n+m} h(n+m) \leqslant h(n), \quad \frac{m}{n+m} h(n+m) \leqslant h(m) .
$$

Таким образом,

$$
h(n+m)=\frac{n}{n+m} h(n+m)+\frac{m}{n+m} h(n+m) \leqslant h(n)+h(m),
$$

т.е. $h$ субаддитивна, и, тем самым, $\Omega$ субмультипликативен. Лемма 46 доказана.

Заметим, что (3.19) вытекает из (3.16). Действительно, так как $h(0)=0$, то в силу (3.16) мы имеем:

$$
h(n)=\sum_{i=1}^{n}[h(i)-h(i-1)] \geqslant n[h(n+1)-h(n)],
$$

откуда вытекает (3.19).

В дальнейшем нам полезно также следующее техническое утверждение.

Лемма 47. Пусть вес $\Omega$ таков, что функиия $h$ в его представлении (3.13) удовлетворяет соотношению

$$
\frac{h(n)}{n} \geqslant \frac{h(n+1)}{n+1} \quad \partial л \Omega \quad n \geqslant n_{0} .
$$

Тогда для всех достаточно мальх $\varepsilon>0$ вес

$$
\Omega_{\varepsilon}(n)=\min \left(e^{\varepsilon|n|}, \Omega(n)\right), \quad n \in \mathbb{Z},
$$

является субмультипликативным.

ДокАЗАТЕЛЬство. Зафиксируем любое $\varepsilon<\min \left\{h(n) / n, 1 \leqslant n \leqslant n_{0}\right\}$. Если $\varepsilon \leqslant h(n) / n$ для всех $n>n_{0}$ (такое может случиться, если $\lim h(n) / n>0$ ), то утверждение выполнено, поскольку $\Omega_{\varepsilon}(n)=\exp (\varepsilon|n|)$. В противном случае для некоторого $n_{1}>n_{0}$ мы имеем: $\varepsilon \leqslant h(n) / n$ для $1 \leqslant n \leqslant n_{1}$ и $\varepsilon>h(n) / n$ для $n>n_{1}$. Таким образом,

$$
\Omega_{\varepsilon}(n)=e^{h_{\varepsilon}(|n|)}, \quad \text { где } \quad h_{\varepsilon}(n)= \begin{cases}\varepsilon n, & 1 \leqslant n \leqslant n_{1}, \\ h(n), & n>n_{1} .\end{cases}
$$

Так как $h_{\varepsilon}(n) / n$ - монотонно убывающая последовательность, то из леммы 46 вытекает, что вес $\Omega_{\varepsilon}-$ субмультипликативный. Лемма 47 доказана.

Лемма 48. Если $\left(x_{k}\right)_{k \in \mathbb{Z}} \in \ell^{2}$, то существует медленно растущий вес $\Omega=$ $(\Omega(k))$ такой, что

$$
\Omega(k) \nearrow \infty, \quad \kappa о г \partial a \quad k \rightarrow \infty, \quad\left(x_{k}\right) \in \ell^{2}(\mathbb{Z}, \Omega) .
$$

ДокАЗАТЕльство. Существует такая последовательность положительных чисел $\left(c_{n}\right)$, что

$$
c_{n} \nearrow \infty \quad \text { и } \sum_{n}\left(\left|x_{-n}\right|^{2}+\left|x_{n}\right|^{2}\right)\left|c_{n}\right|^{2}<\infty .
$$


Конечно, это очевидно, если последовательность $\left(x_{k}\right)$ имеет лишь конечное число ненулевых членов. Поэтому предположим, что эта последовательность $\left(x_{k}\right)$ имеет бесконечно много ненулевых членов, и пусть $\mathscr{E}_{n}=\left(\sum_{|k| \geqslant n}\left|x_{k}\right|^{2}\right)^{1 / 2}$ для $n \in \mathbb{N}$; тогда можно выбрать $c_{n}=\left(\mathscr{E}_{n}\right)^{-1 / 2}$, поскольку

$$
\left(\left|x_{-n}\right|^{2}+\left|x_{n}\right|^{2}\right)\left|c_{n}\right|^{2}=\frac{\left|x_{-n}\right|^{2}+\left|x_{n}\right|^{2}}{\mathscr{E}_{n}} \leqslant \frac{\left(\mathscr{E}_{n}\right)^{2}-\left(\mathscr{E}_{n+1}\right)^{2}}{\mathscr{E}_{n}+\mathscr{E}_{n+1}}=\mathscr{E}_{n}-\mathscr{E}_{n+1} .
$$

По индукции мы определяем медленно растущую неограниченную последовательность $\left(d_{n}\right)_{n \in \mathbb{N}}$, полагая $d_{1}=c_{1}$ и

$$
d_{2 n+1}=d_{2 n}=\min \left(2 d_{n}, c_{2 n}\right)
$$

Тогда $d_{n} \leqslant c_{n}$ для $n \in \mathbb{N}$ и, следовательно, вес $\Omega$, определенный соотношениями

$$
\Omega(0)=1, \quad \Omega(|k|)=1+d_{|k|} \quad \text { для } \quad k \neq 0,
$$

имеет нужные свойства. Лемма 48 доказана.

3.2. Оценки лакун $\left|\gamma_{n}\right|$ снизу. В лемме 40 были получены оценки лакун $\left|\gamma_{n}\right|$ сверху в терминах параметров $\left|\beta_{n}^{ \pm}\right|$. В самосопряженном случае можно оценить лакуны $\left|\gamma_{n}\right|$ и снизу, что приводит нас к теореме 50 - см. ниже.

Мы намерены вывести теорему 50 из следующей технической леммы; она же будет использована и в разделе 4, где мы рассматриваем несамосопряженные операторы Хилла-Шрёдингера и Дирака.

Лемма 49. В обозначениях леммы 40 как в случае Хилла-Шрёдингера, так и в случае Дирака, существует такая последовательность $\delta_{n} \downarrow 0$, ито для достаточно больиих $|n|$ если $\gamma_{n} \neq 0$ u $\beta_{n}^{-}\left(z_{n}^{+}\right) \beta_{n}^{+}\left(z_{n}^{+}\right) \neq 0$, mo

$$
\left|\gamma_{n}\right| \geqslant\left(\frac{2 \sqrt{t_{n}}}{1+t_{n}}-\delta_{n}\right)\left(\left|\beta_{n}^{-}\left(z_{n}^{*}\right)\right|+\left|\beta_{n}^{+}\left(z_{n}^{*}\right)\right|\right),
$$

әде

$$
t_{n}=\left|\beta_{n}^{+}\left(z_{n}^{+}\right)\right| /\left|\beta_{n}^{-}\left(z_{n}^{+}\right)\right| .
$$

ДокАзАтЕльство. По теореме 9 в случае Хилла-Шрёдингера или по теореме 17 в случае Дирака и соответственно по лемме 29 в первом случае и по предложению 35 - во втором, мы имеем:

$$
\sup _{\left[z_{n}^{-}, z_{n}^{+}\right]}\left|\frac{\partial \beta_{n}^{ \pm}}{\partial z}\right| \leqslant \varepsilon_{n}, \quad \varepsilon_{n} \downarrow 0,
$$

где $\left[z_{n}^{-}, z_{n}^{+}\right]$обозначает отрезок с концевыми точками $z_{n}^{-}$и $z_{n}^{+}$. Так как

$$
\beta_{n}^{ \pm}(z)-\beta_{n}^{ \pm}\left(z_{n}^{*}\right)=\int_{z}^{z_{n}^{*}} \frac{d}{d z}\left(\beta_{n}^{ \pm}(z)\right) d z
$$

то из (3.24) вытекает, что

$$
\left|\beta_{n}^{ \pm}(z)-\beta_{n}^{ \pm}\left(z_{n}^{*}\right)\right| \leqslant \varepsilon_{n}\left|z-z_{n}^{*}\right| \leqslant \varepsilon_{n}\left|z_{n}^{+}-z_{n}^{-}\right| \quad \text { для } \quad z \in\left[z_{n}^{-}, z_{n}^{+}\right] .
$$


Таким образом, для $z \in\left[z_{n}^{-}, z_{n}^{+}\right]$мы имеем:

$$
\left|\beta_{n}^{ \pm}\left(z_{n}^{*}\right)\right|-\varepsilon_{n}\left|z_{n}^{+}-z_{n}^{-}\right| \leqslant\left|\beta_{n}^{ \pm}(z)\right| \leqslant\left|\beta_{n}^{ \pm}\left(z_{n}^{*}\right)\right|+\varepsilon_{n}\left|z_{n}^{+}-z_{n}^{-}\right| .
$$

Напомним, что в доказательстве леммы 40 мы использовали обозначение

$$
\zeta_{n}(z)=z-\alpha_{n}(z)
$$

и показали (см. (2.134)) следующее:

$$
\left(1-\varepsilon_{n}\right)\left|z_{n}^{+}-z_{n}^{-}\right| \leqslant\left|\zeta_{n}^{+}-\zeta_{n}^{-}\right| \leqslant\left(1+\varepsilon_{n}\right)\left|z_{n}^{+}-z_{n}^{-}\right|,
$$

где

$$
\zeta_{n}^{+}=\zeta_{n}\left(z_{n}^{+}\right), \quad \zeta_{n}^{-}=\zeta_{n}\left(z_{n}^{-}\right) .
$$

С другой стороны, в силу (2.131) (т.е. в силу основных уравнений (2.23) для Хилла-Шрёдингера и (2.58) для Дирака), мы имеем:

$$
\left(\zeta_{n}^{+}\right)^{2}=\beta_{n}^{+}\left(z_{n}^{+}\right) \beta_{n}^{-}\left(z_{n}^{+}\right), \quad\left(\zeta_{n}^{-}\right)^{2}=\beta_{n}^{+}\left(z_{n}^{-}\right) \beta_{n}^{-}\left(z_{n}^{-}\right),
$$

и, следовательно,

$$
\left(\zeta_{n}^{+}\right)^{2}-\left(\zeta_{n}^{-}\right)^{2}=\int_{z_{n}^{-}}^{z_{n}^{+}} \frac{d}{d z}\left[\beta_{n}^{+}(z) \beta_{n}^{-}(z)\right] d z .
$$

В силу (3.24) и (3.25), мы имеем:

$$
\sup _{\left[z_{n}^{-}, z_{n}^{+}\right]}\left|\frac{d}{d z}\left[\beta_{n}^{+}(z) \beta_{n}^{-}(z)\right]\right| \leqslant \varepsilon_{n}\left(\left|\beta_{n}^{+}\left(z_{n}^{*}\right)\right|+\left|\beta_{n}^{-}\left(z_{n}^{*}\right)\right|+2 \varepsilon_{n}\left|z_{n}^{+}-z_{n}^{-}\right|\right) .
$$

Ввиду (3.26) и (3.27), мы получаем:

$$
\begin{aligned}
\left|\zeta_{n}^{+}+\zeta_{n}^{-}\right| \cdot\left|\zeta_{n}^{+}-\zeta_{n}^{-}\right| & \leqslant \varepsilon_{n}\left(\left|\beta_{n}^{+}\left(z_{n}^{*}\right)\right|+\left|\beta_{n}^{-}\left(z_{n}^{*}\right)\right|+2 \varepsilon_{n}\left|z_{n}^{+}-z_{n}^{-}\right|\right)\left|z_{n}^{+}-z_{n}^{-}\right| \\
& \leqslant \varepsilon_{n}\left(\left|\beta_{n}^{+}\left(z_{n}^{*}\right)\right|+\left|\beta_{n}^{-}\left(z_{n}^{*}\right)\right|+2 \varepsilon_{n}\left|z_{n}^{+}-z_{n}^{-}\right|\right) \frac{\left|\zeta_{n}^{+}-\zeta_{n}^{-}\right|}{1-\varepsilon_{n}}
\end{aligned}
$$

Так как $\varepsilon_{n} \rightarrow 0$, мы можем предполагать, что $\varepsilon_{n}<1 / 2$. Тогда $1 /\left(1-\varepsilon_{n}\right) \leqslant 2$ и из последнего неравенства вытекает:

$$
\left|\zeta_{n}^{+}+\zeta_{n}^{-}\right| \leqslant 2 \varepsilon_{n}\left(\left|\beta_{n}^{+}\left(z_{n}^{*}\right)\right|+\left|\beta_{n}^{-}\left(z_{n}^{*}\right)\right|\right)+2 \varepsilon_{n}\left|z_{n}^{+}-z_{n}^{-}\right| .
$$

В силу (3.23), мы имеем:

$$
\left|\zeta_{n}^{+}\right|=\sqrt{\left|\beta_{n}^{+}\left(z_{n}^{+}\right)\right|\left|\beta_{n}^{-}\left(z_{n}^{+}\right)\right|}=\frac{\sqrt{t_{n}}}{1+t_{n}}\left(\left|\beta_{n}^{+}\left(z_{n}^{+}\right)\right|+\left|\beta_{n}^{-}\left(z_{n}^{+}\right)\right|\right) .
$$

Тогда, ввиду $(3.25)$ (так как $\left.\sqrt{t_{n}} /\left(1+t_{n}\right) \leqslant 1 / 2\right)$, мы получаем:

$$
\left|\zeta_{n}^{+}\right| \geqslant \frac{\sqrt{t_{n}}}{1+t_{n}}\left(\left|\beta_{n}^{+}\left(z_{n}^{*}\right)\right|+\left|\beta_{n}^{-}\left(z_{n}^{*}\right)\right|\right)-\varepsilon_{n}\left|z_{n}^{+}-z_{n}^{-}\right| .
$$


Теперь из соотношений (3.28)-(3.30) вытекает, что

$$
\begin{aligned}
\left|\zeta_{n}^{+}-\zeta_{n}^{-}\right| & =\left|2 \zeta_{n}^{+}-\left(\zeta_{n}^{+}+\zeta_{n}^{-}\right)\right| \geqslant 2\left|\zeta_{n}^{+}\right|-\left|\zeta_{n}^{+}+\zeta_{n}^{-}\right| \\
& \geqslant\left(\frac{2 \sqrt{t_{n}}}{1+t_{n}}-2 \varepsilon_{n}\right)\left(\left|\beta_{n}^{+}\left(z_{n}^{*}\right)\right|+\left|\beta_{n}^{-}\left(z_{n}^{*}\right)\right|\right)-4 \varepsilon_{n}\left|z_{n}^{+}-z_{n}^{-}\right| .
\end{aligned}
$$

Ввиду (3.26), это приводит к неравенству

$$
(1+5 \varepsilon)\left|z_{n}^{+}-z_{n}^{-}\right| \geqslant\left(\frac{2 \sqrt{t_{n}}}{1+t_{n}}-2 \varepsilon_{n}\right)\left(\left|\beta_{n}^{+}\left(z_{n}^{*}\right)\right|+\left|\beta_{n}^{-}\left(z_{n}^{*}\right)\right|\right) .
$$

Поскольку $(1+5 \varepsilon)^{-1} \geqslant 1-5 \varepsilon$ и $2 \sqrt{t_{n}} /\left(1+t_{n}\right) \leqslant 1$, мы получаем, что

$$
\begin{aligned}
\left|\gamma_{n}\right| & =\left|z_{n}^{+}-z_{n}^{-}\right| \geqslant\left(1-5 \varepsilon_{n}\right)\left(\frac{2 \sqrt{t_{n}}}{1+t_{n}}-2 \varepsilon_{n}\right)\left(\left|\beta_{n}^{+}\left(z_{n}^{*}\right)\right|+\left|\beta_{n}^{-}\left(z_{n}^{*}\right)\right|\right) \\
& \geqslant\left(\frac{2 \sqrt{t_{n}}}{1+t_{n}}-7 \varepsilon_{n}\right)\left(\left|\beta_{n}^{+}\left(z_{n}^{*}\right)\right|+\left|\beta_{n}^{-}\left(z_{n}^{*}\right)\right|\right) .
\end{aligned}
$$

Таким образом, (3.22) выполнено, если положить $\delta_{n}=7 \varepsilon_{n}$. Лемма 49 доказана.

ТеОРема 50. Пусть $L$ - самосопряжсенный оператор Хилла-Шрёдингера или Дирака, и пусть $\left(\gamma_{n}\right)$ - последовательность его спектральных лакун. Тогда существует такая последовательность $\delta_{n} \downarrow 0$, что

$$
\left(1-\delta_{n}\right)\left(\left|\beta_{n}^{-}\left(z_{n}^{*}\right)\right|+\left|\beta_{n}^{+}\left(z_{n}^{*}\right)\right|\right) \leqslant\left|\gamma_{n}\right| \leqslant\left(1+\delta_{n}\right)\left(\left|\beta_{n}^{-}\left(z_{n}^{*}\right)\right|+\left|\beta_{n}^{+}\left(z_{n}^{*}\right)\right|\right) .
$$

ДокАзАтельство. Правое неравенство в (3.31) было доказано в лемме 40 для любых потенциалов (даже в несамосопряженном случае).

Так как $L$ - самосопряженный оператор, то мы знаем по части (b) леммы 24 для случая Хилла-Шрёдингера или по части (b) леммы 30 для случая Дирака, что

$$
\left|\beta_{n}^{+}\left(z_{n}^{+}\right)\right|=\left|\beta_{n}^{-}\left(z_{n}^{+}\right)\right|
$$

Если $\left|\beta_{n}^{+}\left(z_{n}^{+}\right)\right|=\left|\beta_{n}^{-}\left(z_{n}^{+}\right)\right| \neq 0$ и $\gamma_{n} \neq 0$, то левое неравенство в (3.31) вытекает немедленно из леммы 49, хотя, быть может, с иной последовательностью $\delta_{n} \downarrow 0$.

Если $\left|\beta_{n}^{+}\left(z_{n}^{+}\right)\right|=\left|\beta_{n}^{-}\left(z_{n}^{+}\right)\right|=0$ при некотором $n$, то $\lambda_{n}^{+}=\lambda^{0}+z_{n}^{+}$- собственное значение геометрической кратности 2 оператора $P^{0} L^{0} P^{0}+S\left(\lambda^{+}\right): E^{0} \rightarrow E^{0}$. Тогда, в силу замечания $22, \gamma_{n}=0$, так что (3.31) выполнено.

Если $\gamma_{n}=0$ при некотором $n$, то, поскольку $L$ самосопряженный, $\lambda_{n}^{+}-$собственное значение оператора $L$ геометрической кратности 2 . Но тогда, в силу замечания $22, \lambda_{n}^{+}$- собственное значение геометрической кратности 2 оператора $P^{0} L^{0} P^{0}+S\left(\lambda^{+}\right)$. Тогда внедиагональные элементы в матричном представлении $S\left(\lambda_{n}^{+}\right)$должны быть нулями, т.е. мы имеем: $\beta_{n}^{+}\left(z_{n}^{+}\right)=\beta_{n}^{-}\left(z_{n}^{+}\right)=0$ и соотношение (3.31) становится тривиальным. Теорема 50 доказана.

\section{3. Гладкость потенциалов Хилла-Шрёдингера в самосопряжен-} ном случае. В этом пункте наша цель - показать (при некоторых ограничениях на вес $\Omega$ ), что если $v$ - вещественнозначный потенциал и $\gamma=\left(\gamma_{n}\right)_{n \in \mathbb{N}}-$ его последовательность спектральных лакун, то

$$
\gamma \in \ell^{2}(\mathbb{N}, \Omega) \Rightarrow v \in H(\Omega) .
$$


Для любого (даже комплекснозначного) потенциала

$$
v \in L^{2}([0, \pi]), \quad v(x)=\sum_{k \in \mathbb{Z}} v_{k} e^{2 i k x},
$$

параметры $\beta_{n}^{ \pm}(v ; z)$ вполне определены по (2.21) и (2.22), если $|n|>n_{0}(\|v\|)$, $|z| \leqslant|n| / 2$.

Если $N>n_{0}(\|v\|)$, положим

$$
\Phi_{N}(v)=\sum_{|n|>N}\left(\beta_{n}^{-}\left(v ; z_{n}^{*}(v)\right) e^{-2 i n x}+\beta_{n}^{+}\left(v ; z_{n}^{*}(v)\right) e^{2 i n x}\right)
$$

где $z_{n}^{*}(v)=\frac{1}{2}\left(\lambda_{n}^{-}(v)+\lambda_{n}^{+}(v)\right)-n^{2}$, и

$$
A_{N}(v)=v+\Phi_{N}(v)
$$

Если $v$ - вещественнозначный потенциал, то, в силу (3.34) и теоремы 50, мы имеем:

$$
\left(\gamma_{n}\right) \in \ell^{2}(\Omega) \Rightarrow\left(\left|\beta_{n}^{-}\left(z_{n}^{*}\right)\right|+\left|\beta_{n}^{+}\left(z_{n}^{*}\right)\right|\right) \in \ell^{2}(\Omega) \Rightarrow A_{N}(v) \in H(\Omega)
$$

для любого субмультипликативного веса $\Omega$. Поэтому соотношение (3.32) будет доказано, если мы покажем, что

$$
A_{N}(v) \in H(\Omega) \Rightarrow v \in H(\Omega) .
$$

Если $v$ - вещественнозначный потенциал, то оператор $L=L^{0}+v$ - самосопряженный, его периодический и антипериодический спектры лежат на вещественной прямой, так что числа $z_{n}^{*}=\frac{1}{2}\left(\lambda_{n}^{+}-\lambda_{n}^{-}\right)-n^{2}-$ вещественные. Следовательно, по второй части леммы 24 и соотношениям (2.32) и (2.33), мы имеем:

$$
\beta_{n}^{-}\left(v ; z_{n}^{*}\right)=\overline{\beta_{n}^{+}\left(v ; z_{n}^{*}\right)}
$$

Таким образом, в силу (3.33) и (3.34) получаем:

$$
v \text { - вещественнозначный } \Rightarrow \Phi_{N}(v), A_{N}(v) \text { - вещественнозначные. }
$$

Пусть $\mathscr{B}_{r}$ обозначает шар

$$
\mathscr{B}_{r}=\left\{v \in L^{2}([0, \pi]):\|v\| \leqslant r\right\} .
$$

Мы рассмотрим также для любого веса веса $\Omega$ соответствующий шар радиуса $r$ :

$$
\mathscr{B}_{r}^{\Omega}=\left\{v \in H(\Omega):\|v\|_{\Omega} \leqslant r\right\} .
$$

Следующая лемма играет решающую роль в доказательстве импликации (3.32) в случае быстро растущих субмультипликативных субэкспоненциальных весов $\Omega$. Более того, она же будет использована позже, в разделе 5 , для доказательства того, что конечнозонные потенциалы плотны в пространстве $H(\Omega)$ при любом субмультипликативном весе $\Omega$. 
Лемма 51. Существуют такая последовательносль положительных чисел $\left(r_{N}\right)_{N \in \mathbb{N}}, r_{N} \nearrow \infty$, и такое $N^{*} \in \mathbb{N}$, что отображение $\Phi_{N}$ вполне определено на шаре $\mathscr{B}_{3 r_{N}}$, если $N \geqslant N^{*} ;$ и если $\Omega$ - субмультипликативный вес на $\mathbb{Z}$, то отображение

$$
\Phi_{N}: \mathscr{B}_{3 r_{N}}^{\Omega} \rightarrow H(\Omega)
$$

вполне определено, если $N \geqslant N_{*}$, и удовлетворяет

$$
\left\|\Phi_{N}\left(v_{1}\right)-\Phi_{N}\left(v_{2}\right)\right\|_{\Omega} \leqslant \frac{1}{2}\left\|v_{1}-v_{2}\right\|_{\Omega}, \quad \text { ecлu } \quad v_{1}, v_{2} \in \mathscr{B}_{r_{N}}^{\Omega}
$$

Более того,

$$
\frac{1}{2}\left\|v_{1}-v_{2}\right\|_{\Omega} \leqslant\left\|A_{N}\left(v_{1}\right)-A_{N}\left(v_{2}\right)\right\|_{\Omega} \leqslant \frac{3}{2}\left\|v_{1}-v_{2}\right\|_{\Omega}, \text { ecлu } v_{1}, v_{2} \in \mathscr{B}_{r_{N}}^{\Omega},
$$

и мы имеем:

$$
A_{N}\left(\mathscr{B}_{r_{N}}^{\Omega}\right) \supset \mathscr{B}_{r_{N} / 2}^{\Omega}
$$

ДокАЗАТЕльство. Все утверждения об операторе Хилла-Шрёдингера, доказанные до настоящего момента (в частности, теорема 9, построения в п. 2.2, предложение 28), выполнены, если $N>N_{*}(\|v\|)=C(1+\|v\|)$, где $C$ - некоторая абсолютная постоянная. Положим

$$
r_{N}=\sqrt{N}, \quad N \in \mathbb{N}
$$

Выберем $N^{*}$ так, что

$$
N>C(1+3 \sqrt{N}) \text { для } N \geqslant N^{*} .
$$

Пусть $N \geqslant N^{*}$. Если $v \in \mathscr{B}_{3 r_{N}}$, то мы имеем

$$
N>C\left(1+3 r_{N}\right) \geqslant C(1+\|v\|)=N_{*}(\|v\|),
$$

и, следовательно, $\beta_{n}^{ \pm}\left(v ; z_{n}^{*}\right)$ вполне определены для $|n|>N$. Ввиду (3.34), это означает, что $\Phi_{N}(v)$ вполне определено на шаре $\mathscr{B}_{3 r_{N}}$.

В силу соотношения (2.52) из предложения 28 , если $v \in H(\Omega)$, то мы имеем:

$$
\left\|\Phi_{N}(v)\right\|_{\Omega}^{2}=\sum_{|n|>N}\left(\left|\beta_{n}^{-}\left(z_{n}^{*}\right)-v_{-n}\right|^{2}+\left|\beta_{n}^{+}\left(z_{n}^{*}\right)-v_{n}\right|^{2}\right)(\Omega(n))^{2} \leqslant \sum_{|n|>N} \frac{16}{n^{2}}\|v\|_{\Omega}^{4} .
$$

Следовательно,

$$
\left\|\Phi_{N}(v)\right\|_{\Omega} \leqslant \frac{8}{N}\|v\|_{\Omega}^{2}
$$

С другой стороны, $\Phi_{N}(v)$ зависит аналитически от $v \in B_{3 r_{N}}$ (т.е. ограничение $\Phi_{N}$ на любой комплексный круг, лежащий в шаре $B_{3 r_{N}}$, аналитично). Действительно, по п. 2.2 (см. (2.21) и (2.95) или (2.101)) параметры $\widetilde{\beta}(v ; n, z)$ зависят аналитически от $v \in B_{3 r_{N}}$, если $|z| \leqslant|N| / 2$. Следовательно, ввиду (3.34), нам надо только объяснить, что параметры

$$
z_{n}^{*}(v)=\frac{\lambda_{n}^{-}(v)+\lambda_{n}^{+}(v)}{2}-n^{2}, \quad n \in \mathbb{Z},
$$


зависят аналитически от $v \in B_{3 r_{N}}$. По теореме $9, \lambda_{n}^{ \pm}$расположено в круге $D\left(n^{2} ; r\right)$ с центром $n^{2}$ и радиусом $r=r(\|v\|)$; более того, нет никаких других собственных значений оператора $L$ внутри круга $D\left(n^{2} ; n / 2\right)$. Поэтому мы можем выбрать радиус $\rho$ так, что круг $D\left(n^{2} ; \rho\right)$ содержит $\lambda_{n}^{ \pm}(v)$, но не содержит никаких других собственных значений оператора $L=L(v)$, если $v \in \mathscr{B}_{r_{N}}^{\Omega}$. Следовательно, ввиду леммы 21, мы имеем:

$$
\lambda_{n}^{-}(v)+\lambda_{n}^{+}(v)=\operatorname{Trace}\left\{\frac{1}{2 \pi i} \int_{\partial D\left(n^{2}, \rho\right)} \lambda\left[\lambda-\left(L^{0}+S_{\lambda}(n ; v)\right)\right]^{-1} d \lambda\right\} .
$$

Так как подынтегральная функция зависит аналитически от $v \in B_{3 r_{N}}$, то мы получаем нужное утверждение.

Теперь мы докажем (3.38). Зафиксируем $v_{1}$ и $w$ так, что $\left\|v_{1}\right\|_{\Omega} \leqslant r_{N}$ и $\|w\|_{\Omega}=1$. Поскольку функция

$$
t \rightarrow \Phi_{N}\left(v_{1}+t w\right)
$$

аналитична в круге $|t| \leqslant 2 r_{N}$, то в силу (3.41) неравенство Коши для первой производной дает:

$$
\sup _{|t| \leqslant r_{N}}\left\|\frac{d}{d t} \Phi_{N}\left(v_{1}+t w\right)\right\|_{\Omega} \leqslant \frac{1}{r_{N}} \sup _{|s| \leqslant 2 r_{N}}\left\|\Phi_{N}\left(v_{1}+s w\right)\right\| \leqslant \frac{1}{r_{N}} \cdot \frac{8\left(3 r_{N}\right)^{2}}{N} \leqslant \frac{1}{2} .
$$

Таким образом, если $\left\|v_{1}-v_{2}\right\|_{\Omega} \leqslant r_{N}$, то для $w=\left(v_{2}-v_{1}\right) /\left\|v_{2}-v_{1}\right\|_{\Omega}$ мы имеем:

$$
\left\|\Phi_{N}\left(v_{1}\right)-\Phi_{N}\left(v_{2}\right)\right\|_{\Omega} \leqslant \sup _{|t| \leqslant r_{N}}\left\|\frac{d}{d t} \Phi_{N}\left(v_{1}+t w\right)\right\|_{\Omega} \cdot\left\|v_{1}-v_{2}\right\|_{\Omega} \leqslant \frac{1}{2}\left\|v_{1}-v_{2}\right\|_{\Omega} .
$$

Если $v_{1}, v_{2} \in \mathscr{B}_{r_{N}}^{\Omega}$, но $\left\|v_{1}-v_{2}\right\|_{\Omega}>r_{N}$, то (3.41) влечет за собой, что

$$
\begin{aligned}
\left\|\Phi_{N}\left(v_{1}\right)-\Phi_{N}\left(v_{2}\right)\right\|_{\Omega} & \leqslant\left\|\Phi_{N}\left(v_{1}\right)\right\|_{\Omega}+\left\|\Phi_{N}\left(v_{2}\right)\right\|_{\Omega} \leqslant \frac{8\left\|v_{1}\right\|_{\Omega}^{2}}{N}+\frac{8\left\|v_{2}\right\|_{\Omega}^{2}}{N} \\
& \leqslant \frac{16 r_{N}}{N}\left\|v_{1}-v_{2}\right\|_{\Omega} \leqslant \frac{1}{2}\left\|v_{1}-v_{2}\right\|_{\Omega},
\end{aligned}
$$

и, следовательно, соотношение (3.38) выполнено.

Конечно, из (3.38) вытекает (3.39), поскольку

$$
\begin{aligned}
\left\|v_{1}-v_{2}\right\|_{\Omega} & =\left\|A_{N}\left(v_{1}\right)-A_{N}\left(v_{2}\right)-\left(\Phi\left(v_{1}\right)-\Phi\left(v_{2}\right)\right)\right\|_{\Omega} \\
& \leqslant\left\|A_{N}\left(v_{1}\right)-A_{N}\left(v_{2}\right)\right\|_{\Omega}+\frac{1}{2}\left\|v_{1}-v_{2}\right\|_{\Omega} .
\end{aligned}
$$

Наконец, стандартное рассуждение показывает, что из (3.38) вытекает (3.40). Действительно, для каждого $u \in \mathscr{B}_{r_{N} / 2}^{\Omega}$ оператор $v \mapsto u-\Phi_{N}(v)$ отображает $\mathscr{B}_{r_{N}}^{\Omega}$ в себя, поскольку $\Phi_{N}(0)=0$. Следовательно,

$$
\left\|\Phi_{N}(v)\right\|_{\Omega}=\left\|\Phi_{N}(v)-\Phi_{N}(0)\right\|_{\Omega} \leqslant \frac{1}{2}\|v-0\|_{\Omega} \leqslant \frac{1}{2} r_{N} .
$$

Таким образом, принцип сжимающих отображений показывает, что этот оператор имеет единственную неподвижную точку $v \in \mathscr{B}_{r_{N}}^{\Omega}$, т.е. $A_{N}(v)=u$ в точности для одного $v \in \mathscr{B}_{r_{N}}^{\Omega}$. Лемма 51 доказана. 
ЗАмечАниЕ 52. Лемма 51 сформулирована и доказана для пространств комплекснозначных потенциалов, но структура формул в (3.37) непосредственно показывает, что она выполнена и тогда, когда мы рассматриваем только вещественнозначные потенциалы.

ПРЕДЛОЖЕНИЕ 53. Пусть вес $\Omega=(\Omega(n))_{n \in \mathbb{Z}}$ maков, что

$$
\frac{\log \Omega(n)}{n} \searrow 0, \quad \text { когда } n \rightarrow \infty
$$

Тогда существует такое $N^{*} \in \mathbb{N}$, ито для любого $v \in L^{2}([0, \pi])$ мъь имеем:

$$
A_{N}(v) \in H(\Omega) \quad \forall N>N^{*} \Rightarrow v \in H(\Omega) .
$$

Если $\Omega$ - субмультипликативный вес экспоненциального типа, то

$$
A_{N}(v) \in H(\Omega) \quad \forall N>N^{*} \Rightarrow \exists \varepsilon>0: \quad v \in H\left(e^{\varepsilon|n|}\right) .
$$

ДокАЗАТЕЛЬСтво. По лемме 47, для любого $\varepsilon>0$ вес

$$
\Omega_{\varepsilon}(m)=\min \left(e^{\varepsilon|m|}, \Omega(m)\right)
$$

субмультипликативный, и, более того, $H\left(\Omega_{\varepsilon}\right)=H(\Omega)$. Следовательно, для каждого $\varepsilon>0$ мы имеем: $A_{N}(v) \in H\left(\Omega_{\varepsilon}\right)$, и теперь достаточно показать, что $v \in H\left(\Omega_{\varepsilon}\right)$ при некотором $\varepsilon>0$.

Если $A_{N}(v) \in H(\Omega)$ для всех $N>N^{*}$, то, ввиду леммы 44 и неравенства (3.41), мы можем выбрать $N>N^{*}$ так, что

$$
\left\|A_{N}(v)\right\| \leqslant\|v\|+\left\|\Phi_{N}(v)\right\| \leqslant\|v\|+8\|v\|^{2} / N \leqslant r_{N} / 8 .
$$

Зафиксируем такое $N$; тогда

$$
w:=A_{N}(v) \in H(\Omega), \quad\|w\| \leqslant r_{N} / 8 .
$$

Существует такое $\varepsilon>0$, что $\|w\|_{\Omega_{\varepsilon}} \leqslant r_{N} / 2$. Действительно, пусть $w(x)=$ $\sum_{k \in \mathbb{Z}} w_{k} \exp (2 i k x)$; выберем $N_{1} \in \mathbb{N}$ так, что

$$
\sum_{|k|>N_{1}}\left|w_{k}\right|^{2}(\Omega(k))^{2}<\frac{r_{N}^{2}}{16}
$$

После этого выберем $\varepsilon>0$ так, что $e^{\varepsilon N_{1}} \leqslant 2$. Тогда мы имеем:

$$
\Omega_{\varepsilon}(m) \leqslant 2, \text { если }|m| \leqslant N_{1} .
$$

Из (3.45)-(3.47) вытекает, что

$$
\|w\|_{\Omega_{\varepsilon}}^{2} \leqslant \sum_{|k| \leqslant N_{1}} 4\left|w_{k}\right|^{2}+\sum_{|k|>N_{1}}\left|w_{k}\right|^{2}(\Omega(k))^{2} \leqslant 4\|w\|^{2}+\frac{r_{N}^{2}}{16} \leqslant \frac{r_{N}^{2}}{16}+\frac{r_{N}^{2}}{16}
$$

и, следовательно, $\|w\|_{\Omega_{\varepsilon}}<r_{N} / 2$. 
По лемме 51 , существует $\widetilde{v} \in \mathscr{B}_{r_{N}}^{\Omega_{\varepsilon}} \subset \mathscr{B}_{r_{N}}$ такая, что

$$
A_{N}(\widetilde{v})=w=A_{N}(v) .
$$

С другой стороны, по лемме 51 , ограничение отображения $A_{N}$ на шар $\mathscr{B}_{r_{N}}$ инъективно. Таким образом,

$$
v=\widetilde{v} \in H\left(\Omega_{\varepsilon}\right)=H(\Omega) .
$$

Если $\Omega$ - субмультипликативный вес такой, что выполнено (3.50) (см. ниже), то легко видеть, что для всякого достаточно малого $\varepsilon>0$ мы имеем:

$$
\Omega_{\varepsilon}(m)=\min \left(e^{\varepsilon|m|}, \Omega(m)\right) \equiv e^{\varepsilon|m|} .
$$

Поэтому то же рассуждение показывает, что $v \in H\left(\Omega_{\varepsilon}\right)=H\left(e^{\varepsilon|m|}\right)$. Это завершает доказательство предложения 53.

Tеорема 54. Пусть $L=L^{0}+v(x)$ - оператор Хилла-Шрёдингера с вещественнозначным потенциалом $v \in L^{2}([0, \pi])$, и пусть $\gamma=\left(\gamma_{n}\right)$ - его последовательность спектральных лакун. Если $\Omega=(\Omega(n))_{n \in \mathbb{Z}}-$ субмультипликативный вес такой, что

$$
\frac{\log \Omega(n)}{n} \searrow 0, \quad \text { когда } n \rightarrow \infty
$$

mo

$$
\gamma \in \ell^{2}(\mathbb{N}, \Omega) \Rightarrow v \in H(\Omega)
$$

Если $\Omega$ - субмультипликативный вес экспоненциалъного типа, т.е.

$$
\lim _{n \rightarrow \infty} \frac{\log \Omega(n)}{n}>0,
$$

то существует такое $\varepsilon>0$, что

$$
\gamma \in \ell^{2}(\mathbb{N}, \Omega) \Rightarrow v \in H\left(e^{\varepsilon|n|}\right) .
$$

ДокАЗАТЕЛЬство. По теореме 50, если $v$ - вещественнозначный потенциал, то для любого субмультипликативного веса $\Omega$ мы имеем:

$$
\left(\gamma_{n}\right) \in \ell^{2}(\Omega) \Rightarrow A_{N}(v)=v+\Phi_{N}(v) \in H(\Omega) .
$$

Таким образом, теорема 54 вытекает из предложения 53.

3.4. Гладкость потенциалов Дирака в самосопряженном случае. Соотношения (2.80) определяют параметры $\beta^{ \pm}$для любого потенциала Дирака $v(x)=\left(\begin{array}{cc}0 & P(x) \\ Q(x) & 0\end{array}\right)$, где

$$
P(x)=\sum_{k \in \mathbb{Z}} P_{k} e^{2 i k x}, \quad Q(x)=\sum_{k \in \mathbb{Z}} Q_{k} e^{2 i k x},
$$

если $|n| \geqslant N_{*}(v),|z| \leqslant 1$. 
Теперь для достаточно больших $N \in \mathbb{N}$ положим

$$
\Phi_{N}(v)=\left(\begin{array}{cc}
0 & \Phi_{N}^{12}(v) \\
\Phi_{N}^{21}(v) & 0
\end{array}\right), \quad A_{N}(v)=v+\Phi_{N}(v),
$$

где

$$
\begin{aligned}
\Phi_{N}^{12}(v) & =\sum_{|n|>N}\left(\beta_{n}^{-}\left(v ; z_{n}^{*}\right)-P_{-n}\right) e^{-2 i n x} \\
\Phi_{N}^{21}(v) & =\sum_{|n|>N}\left(\beta_{n}^{+}\left(v ; z_{n}^{*}\right)-Q_{n}\right) e^{2 i n x}
\end{aligned}
$$

и

$$
z_{n}^{*}=\frac{\lambda_{n}^{-}(v)+\lambda_{n}^{+}(v)}{2}-n
$$

В самосопряженном случае, когда $\overline{Q(x)}=P(x)$, по теореме 50 мы имеем:

$$
\left(\gamma_{n}\right) \in \ell^{2}(\Omega) \Rightarrow A_{N}(v) \in H_{D}(\Omega)
$$

Наша цель - показать (при некоторых ограничениях на вес $\Omega$ ), что

$$
\left(\gamma_{n}\right) \in \ell^{2}(\Omega) \Rightarrow v \in H_{D}(\Omega) .
$$

Ввиду (3.55), достаточно доказать, что

$$
v+\Phi_{N}(v) \in H_{D}(\Omega) \Rightarrow v \in H_{D}(\Omega) .
$$

Мы говорим, что потенциал $v(x)=\left(\begin{array}{cc}0 & P(x) \\ Q(x) & 0\end{array}\right)$ - самосопряженный, если $Q(x)=\overline{P(x)}$. Если $v$ - самосопряженный потенциал, то соответствующий опеpaтор $L=L^{0}+v$ - самосопряженный, его периодический и антипериодический спектры лежат на вещественной прямой, так что числа $z_{n}^{*}=\frac{1}{2}\left(\lambda_{n}^{+}-\lambda_{n}^{-}\right)-n$ вещественны. Следовательно, в силу второй части леммы 30 и соотношений (2.79), (2.80), мы имеем:

$$
\beta_{n}^{-}\left(v ; z_{n}^{*}\right)=\overline{\beta_{n}^{+}\left(v ; z_{n}^{*}\right)} .
$$

Таким образом, ввиду (3.52)-(3.54),

$$
v \text { самосопряженный } \Rightarrow \Phi_{N}(v), A_{N}(v) \text { самосопряженные. }
$$

Для всякого веса $\Omega$ мы обозначим через $\mathscr{B}_{r}^{\Omega}$ шар

$$
\mathscr{B}_{r}^{\Omega}=\left\{v \in H_{D}(\Omega):\|v\|_{\Omega} \leqslant r\right\} .
$$

Следующая лемма играет решающую роль в доказательстве соотношения (3.56) в случае быстро растущих субмультипликативных субэкспоненциальных весов $\Omega$. Более того, она же будет использована позже, в разделе 5 , в доказательстве того, что конечнозонные потенциалы плотны в пространстве $H_{D}(\Omega)$ в случае любого субмультипликативного веса $\Omega$. 
Лемма 55. Пусть $\Omega_{1}$ - медленно растущий неограниченный вес такой, чmo

$$
\Omega_{1}(k) \leqslant C_{1}|k| .
$$

Тогда существуют последовательность положительных чисел $\left(r_{N}\right)_{N \in \mathbb{N}}$, $r_{N} \nearrow \infty$, и натуральное $N^{*}=N^{*}\left(\Omega_{1}\right) \in \mathbb{N}$ такие, что отображение $\Phi_{N}$ вполне определено на шаре $\mathscr{B}_{3 r_{N}}^{\Omega_{1}}$ для всех $N>N_{*}$.

Более того, если $\Omega=(\Omega(k))_{k \in \mathbb{Z}}-$ вес вида

$$
\Omega(k)=\Omega_{1}(k) \Omega_{2}(k), \quad k \in \mathbb{Z},
$$

где $\Omega_{2}$ - субмультипликативньй вес, то отображсение $\Phi_{N}: \mathscr{B}_{3 r_{N}}^{\Omega} \rightarrow H_{D}(\Omega)$ вполне определено, если $N>N^{*}$, и имеет следующие свойства:

$$
\begin{gathered}
\left\|\Phi_{N}\left(v_{1}\right)-\Phi_{N}\left(v_{2}\right)\right\|_{\Omega} \leqslant \frac{1}{2}\left\|v_{1}-v_{2}\right\|_{\Omega}, \quad \text { ecлu } \quad v_{1}, v_{2} \in \mathscr{B}_{r_{N}}^{\Omega}, \\
\frac{1}{2}\left\|v_{1}-v_{2}\right\|_{\Omega} \leqslant\left\|A_{N}\left(v_{1}\right)-A_{N}\left(v_{2}\right)\right\|_{\Omega} \leqslant \frac{3}{2}\left\|v_{1}-v_{2}\right\|_{\Omega}, \quad \text { ecлu } \quad v_{1}, v_{2} \in \mathscr{B}_{r_{N}}^{\Omega}, \\
A_{N}\left(\mathscr{B}_{r_{N}}^{\Omega}\right) \supset \mathscr{B}_{r_{N} / 2}^{\Omega} .
\end{gathered}
$$

ДокАЗАТЕльство. Так как доказательство аналогично доказательству леммы 51, мы опускаем многие детали.

По теореме 17 , для каждого $L^{2}$-потенциала Дирака $v$ существует такое $N_{*}=$ $N_{*}(v)$, что если $|n|>N_{*}$, то оператор Дирака $L=L^{0}+v$ имеет два (с учетом их кратности, периодических для четных $n$ и антипериодических для нечетных $n)$ собственных значения $\lambda_{n}^{-}(v)$ и $\lambda_{n}^{+}(v)$ в круге $D(n ; 1 / 8)$ с центром $n$ и радиусом $1 / 8$.

Более того, можно выбрать $N_{*}$ зависящим только от нормы $\|v\|_{\Omega_{1}}$. Действительно, в доказательстве теоремы 17 мы выбираем $N_{*}$ так, что (1.159) выполнено, т.е.

$$
\|v\| C \delta(n ; 1 / 8) \leqslant 1 / 2, \quad \text { если }|n| \geqslant N_{*},
$$

где $C$ - абсолютная постоянная, и, в силу (1.154), если $w=v$, то

$$
\delta(n ; 1 / 8)=\frac{\|v\|}{\sqrt{|n|}}+\mathscr{E}_{|n| / 2}(v) .
$$

Так как $\Omega_{1}$ - медленно растущий вес, то существует постоянная $C_{0}=C_{0}\left(\Omega_{1}\right)$ такая, что

$$
\Omega_{1}(2 k) \leqslant C_{0} \Omega_{1}(k), \quad k \in \mathbb{Z}
$$

Следовательно,

$$
\left(\mathscr{E}_{|n| / 2}(v)\right)^{2}=\sum_{|k| \geqslant|n| / 2}\left(\left|P_{k}\right|^{2}+\left|Q_{k}\right|^{2}\right)^{2} \leqslant \frac{C_{0}^{2}}{\left(\Omega_{1}(n)\right)^{2}} \sum_{|k| \geqslant|n| / 2}\left(\left|P_{k}\right|^{2}+\left|Q_{k}\right|^{2}\right)^{2}\left|\Omega_{1}(k)\right|^{2},
$$

и мы получаем:

$$
\mathscr{E}_{|n| / 2}(v) \leqslant \frac{C_{0}}{\Omega_{1}(n)}\|v\|_{\Omega_{1}}
$$


Таким образом,

$$
\|v\| C \delta(n ; 1 / 8) \leqslant C\|v\|_{\Omega_{1}}^{2}\left(\frac{1}{\sqrt{|n|}}+\frac{1}{\sqrt{\Omega_{1}(n)}}\right),
$$

где $C=C\left(\Omega_{1}\right)$, и поэтому (3.63) будет выполнено, если $N_{*}=N_{*}\left(\|v\|_{\Omega_{1}}\right)$ выбрано так, что

$$
C\|v\|_{\Omega_{1}}^{2}\left(\frac{1}{\sqrt{|n|}}+\frac{1}{\sqrt{\Omega_{1}(n)}}\right) \leqslant \frac{1}{2} \quad \text { для }|n| \geqslant N_{*} .
$$

$\mathrm{C}$ другой стороны, по конструкциям п. 2.3, параметры $\beta^{ \pm}(v ; n, z)$ вполне определены соотношением (2.80), если $\left\|T_{n}^{2}\right\| \leqslant 1 / 2$, и $T_{n}$ определено соотношением (2.55). Ввиду $(2.92),(2.93)$ и (2.103) в лемме 33 , нужное неравенство будет выполнено, если

$$
C\left(\mathscr{E}_{|n|}(v)+\frac{\|v\|}{\sqrt{|n|}}\right)\|v\| \leqslant 1 / 2,
$$

что приводит нас к тому же условию (3.64), хотя, может быть, с иной постоянной $C$. Таким образом, если (3.64) выполнено, то отображение $\Phi_{N}(v)$ вполне определено, и в силу соотношения (2.128) в предложении 39 мы имеем:

$$
\left\|\Phi_{N}(v)\right\|_{\Omega}^{2} \leqslant C\left(\frac{1}{\left(\Omega_{1}(N)\right)^{2}}+\frac{1}{N}\right)\|v\|_{\Omega}^{6},
$$

где $C=C\left(\Omega_{1}\right)$.

Положим

$$
r_{N}=(3 C)^{-1 / 6}\left(\frac{1}{\Omega_{1}(N)}+\frac{1}{N}\right)^{-1 / 6},
$$

где $C$ - максимальная из постоянных, появляющихся в (3.64) и (2.128).

Так как $r_{N} \nearrow \infty$ и

$$
\left(r_{N}\right)^{2}\left(\frac{1}{\Omega_{1}(N)}+\frac{1}{N}\right) \rightarrow 0, \quad \text { когда } \quad N \rightarrow \infty,
$$

то существует $N_{*}=N_{*}\left(\Omega_{1}\right)$ такое, что (3.64) выполнено, если $v \in \mathscr{B}_{3 r_{N}}^{\Omega_{1}}$. Таким образом, в силу (3.65), отображение $\Phi_{N}: \mathscr{B}_{3 r_{N}}^{\Omega} \rightarrow H_{D}(\Omega)$ вполне определено, если $N \geqslant n_{0}$. Более того, ввиду (3.64) и (3.66), мы имеем:

$$
\left\|\Phi_{N}(v)\right\|_{\Omega} \leqslant 1, \quad \text { если } v \in \mathscr{B}_{3 r_{N}}^{\Omega} .
$$

То же рассуждение, что было использовано в доказательстве леммы 51, показывает, что $\Phi_{N}(v)$ зависит аналитически от $v \in \mathscr{B}_{3 r_{N}}^{\Omega}$. Мы опускаем доказательство соотношений (3.60)-(3.62), поскольку оно аналогично доказательству соответствующих утверждений в лемме 51. Лемма 55 доказана.

ЗАмЕчАНИЕ 56. Лемма 55 сформулирована и доказана для пространств произвольных потенциалов, но (3.57) непосредственно показывает, что она верна, если мы говорим только о самосопряженных потенциалах. 
ПРЕДЛОЖЕНИЕ 57. Пусть $\Omega=(\Omega(k))_{k \in \mathbb{Z}}-$ вес вида

$$
\Omega(k)=\Omega_{1}(k) \Omega_{2}(k), \quad k \in \mathbb{Z},
$$

где $\Omega_{1}$ - медленно растущий неограниченный вес такой, что

$$
\Omega_{1}(k) \leqslant C_{1}|k|,
$$

a $\Omega_{2}$ удовлетворяет соотношению

$$
\frac{\log \Omega_{2}(n)}{n} \searrow 0, \quad \kappa о г \partial а \quad n \rightarrow \infty .
$$

Тогда существует такое натуральное $N^{*}=N^{*}\left(\Omega_{1}\right)$, что для $v \in H_{D}\left(\Omega_{1}\right)$ мъь имеем:

$$
A_{N}(v) \in H_{D}(\Omega) \quad \forall N>N^{*} \Rightarrow v \in H_{D}(\Omega) .
$$

Если $\Omega_{2}$ - субмультипликативный вес экспоненциального типа, т.е.

$$
\lim _{n \rightarrow \infty} \frac{\log \Omega_{2}(n)}{n}>0
$$

mo

$$
A_{N}(v) \in H(\Omega) \quad \forall N>N^{*} \Rightarrow \exists \varepsilon>0: \quad v \in H\left(e^{\varepsilon|n|}\right) .
$$

ДокАЗАТЕЛЬСтво. Ввиду леммы 43, можно без ограничения общности предполагать, что $\log \left(\Omega_{1}(n)\right) / n \searrow 0$, когда $n \rightarrow \infty$. Таким образом, мы можем предполагать, что

$$
\frac{\log \Omega(n)}{n} \searrow 0, \quad \text { когда } n \rightarrow \infty .
$$

Тогда, по лемме 43, вес

$$
\Omega^{\varepsilon}(m)=\min \left(e^{\varepsilon|n|}, \Omega(m)\right), \quad \varepsilon>0,
$$

- субмультипликативный, и, более того,

$$
H_{D}\left(\Omega^{\varepsilon}\right)=H_{D}(\Omega) .
$$

Поэтому достаточно показать, что $v \in H_{D}\left(\Omega^{\varepsilon}\right)$ при каком-то $\varepsilon>0$.

Теперь мы воспользуемся леммой 55 и ее обозначениями. Выберем $N>$ $N_{*}\left(\Omega_{1}\right)$ так, что $\|v\|_{\Omega_{1}}<r_{N} / 8$, и положим

$$
w:=A_{N}(v)=v+\Phi_{N}(v) .
$$

Тогда, в силу (3.60) (так как $\Phi_{N}(0)=0$, если мы выбираем $\Omega_{2} \equiv 1$ в (3.59)), мы имеем:

$$
\left\|\Phi_{N}(v)\right\|_{\Omega_{1}} \leqslant \frac{1}{2}\|v\|_{\Omega_{1}} \leqslant \frac{r_{N}}{16}
$$

и, следовательно,

$$
\|w\|_{\Omega_{1}}=\left\|A_{N}(v)\right\|_{\Omega_{1}} \leqslant\|v\|_{\Omega_{1}}+\left\|\Phi_{N}(v)\right\|_{\Omega_{1}} \leqslant \frac{r_{N}}{4} .
$$


В силу (3.70), мы имеем: $w \in H_{D}(\Omega)$. Существует $\varepsilon>0$ такое, что

$$
\|w\|_{\Omega^{\varepsilon}} \leqslant r_{N} / 2
$$

Действительно, пусть

$$
w(x)=\left(\begin{array}{cc}
0 & w^{12} \\
w^{21} & 0
\end{array}\right), w^{12}(x)=\sum_{k \in \mathbb{Z}} w_{k}^{12} \exp (2 i k x), w^{21}(x)=\sum_{k \in \mathbb{Z}} w_{k}^{21} \exp (2 i k x) .
$$

Выберем $N_{1} \in \mathbb{N}$ так, что

$$
\sum_{|k|>N_{1}}\left(\left|w_{k}^{12}\right|^{2}+\left|w_{k}^{21}\right|^{2}\right)(\Omega(k))^{2}<\frac{r_{N}^{2}}{16}
$$

После этого выберем $\varepsilon>0$ так, что $e^{\varepsilon N_{1}} \leqslant \sqrt{2}$. Теперь мы имеем:

$$
\Omega^{\varepsilon}(m) \leqslant \sqrt{2}, \text { если }|m| \leqslant N_{1},
$$

и соотношения (3.74)-(3.76) влекут за собой, что

$$
\begin{aligned}
\|w\|_{\Omega^{\varepsilon}}^{2} & \leqslant \sum_{|k| \leqslant N_{1}} 2\left(\left|w_{k}^{12}\right|^{2}+\left|w_{k}^{21}\right|^{2}\right)+\sum_{|k|>N_{1}}\left(\left|w_{k}^{12}\right|^{2}+\left|w_{k}^{21}\right|^{2}\right)(\Omega(k))^{2} \\
& \leqslant 2\|w\|^{2}+\frac{r_{N}^{2}}{16} \leqslant 2 \frac{r_{N}^{2}}{16}+\frac{r_{N}^{2}}{16}
\end{aligned}
$$

и, следовательно, $\|w\|_{\Omega^{\varepsilon}}<r_{N} / 2$.

По соотношению (3.62) в лемме 55 , существует потенциал $\widetilde{v} \in \mathscr{B}_{r_{N}}^{\Omega^{\varepsilon}} \subset \mathscr{B}_{r_{N}}^{\Omega_{1}}$ такой, что

$$
A_{N}(\widetilde{v})=w=A_{N}(v)
$$

С другой стороны, по соотношению (3.61) в лемме 55, ограничение $A_{N}$ на шар $\mathscr{B}_{r_{N}}^{\Omega_{1}}$ инъективно. Таким образом,

$$
v=\widetilde{v} \in H_{D}\left(\Omega^{\varepsilon}\right)=H_{D}(\Omega) .
$$

Если $\Omega_{2}$ - субмультипликативный вес такой, что (3.71) выполнено, то для всех достаточно малых $\varepsilon>0$ мы имеем:

$$
\Omega^{\varepsilon}(m)=\min \left(e^{\varepsilon|m|}, \Omega(m)\right) \equiv e^{\varepsilon|m|} .
$$

Тогда то же рассуждение, что и выше, показывает, что $v \in H_{D}\left(\Omega^{\varepsilon}\right)=H_{D}\left(e^{\varepsilon|m|}\right)$. Это завершает доказательство предложения 57.

TEOPEMA 58. Пусть

$$
L=L^{0}+v(x), \quad L^{0}=i\left(\begin{array}{cc}
1 & 0 \\
0 & -1
\end{array}\right) \frac{d}{d x}, \quad v(x)=\left(\begin{array}{cc}
0 & P(x) \\
Q(x) & 0
\end{array}\right)
$$

- самосопряженный периодический оператор Дирака (m.е. $P$ и $Q$ - периодические $L^{2}([0,1])$-функиии такие, что $\left.Q(x)=\overline{P(x)}\right)$, и пусть $\gamma=\left(\gamma_{n}\right)_{n \in \mathbb{Z}}-$ его 
последовательность спектральных лакун. Если $\Omega=(\Omega(n))_{n \in \mathbb{Z}}-$ субмультипликативный вес такой, что

$$
\frac{\log \Omega(n)}{n} \searrow 0, \quad \text { когда } n \rightarrow \infty,
$$

mo

$$
\gamma \in \ell^{2}(\mathbb{Z}, \Omega) \Rightarrow v \in H_{D}(\Omega)
$$

Если $\Omega$ - субмультипликативный вес экспоненциального типа, т.е.

$$
\lim _{n \rightarrow \infty} \frac{\log \Omega(n)}{n}>0,
$$

то существует такое $\varepsilon>0$, что

$$
\gamma \in \ell^{2}(\mathbb{Z}, \Omega) \Rightarrow v \in H_{D}\left(e^{\varepsilon|n|}\right) .
$$

ДокАЗАтЕЛьство. Рассмотрим последовательность $x=\left(x_{n}\right)_{n \in \mathbb{Z}}$, где

$$
x_{m}=\left(\left|P_{n}\right|^{2}+\left|Q_{n}\right|^{2}+\left|\gamma_{n}\right|^{2}[\Omega(n)]^{2}\right)^{1 / 2},
$$

а $P_{n}$ и $Q_{n}-$ коэффициенты Фурье функций $P$ и $Q$. Так как $x \in \ell^{2}(\mathbb{Z})$, то, по лемме 48 , существует медленно растущий неограниченный вес $\Omega_{1}$ такой, что $x \in \ell^{2}\left(\mathbb{Z}, \Omega_{1}\right)$. Без потери общности мы можем предполагать, что

$$
\Omega_{1}(n) \leqslant C_{1}|n|
$$

(иначе, ввиду (3.7), мы могли бы заменить $\Omega_{1}$ на $\left(\Omega_{1}\right)^{1 / a}$, где $a$ - соответственно подобранная постоянная). Так как всякий медленно растущий вес эквивалентен субмультипликативному весу, то мы можем также предполагать, что $\Omega_{1}-$ субмультипликативный вес.

В силу выбора $\Omega_{1}$, мы имеем: $\left(\gamma_{n}\right) \in \ell^{2}\left(\Omega_{1} \cdot \Omega\right)$. Так как оператор $L-$ самосопряженный, то из теоремы 50 вытекает, что

$$
\left(\gamma_{n}\right) \in \ell^{2}\left(\Omega_{1} \cdot \Omega\right) \Rightarrow A_{N}(v) \in H_{D}\left(\Omega_{1} \cdot \Omega\right) .
$$

Теперь предложение 57 влечет за собой:

$$
A_{N}(v) \in H_{D}\left(\Omega_{1} \cdot \Omega\right) \Rightarrow v \in H_{D}\left(\Omega_{1} \cdot \Omega\right) \subset H_{D}(\Omega),
$$

и это завершает доказательство.

3.5. Примечания. Веса и весовые пространства коэффициентов Фурье используются в анализе для характеризации гладкости функций очень давно (см. [53], [54]). Конечно, в п. 3.1 мы даем элементарные утверждения в форме, необходимой для дальнейшего. Отметим, что лемма 47, подмеченная в [55], играет существенную техническую роль в дальнейшем. Оценки в п. 3.2 приходят из [38].

Теорема 54 (о случае Хилла-Шрёдингера) - это уточненная форма основного результата работы [38] (см. теорему 10 там), а теорема 58 анонсирована в [18] и доказана в [19] (см. теорему 11 там). Хотя основная схема в настоящей 
статье сохраняется, мы теперь используем прием балансирования "головы" и "хвоста" (т.е. удачный подбор $N_{*}$ и $N$ в предложениях 53 и 57) и построение вспомогательных сжимающих отображений $A_{N}$ (леммы 51 и 55). Такой прием - но только в случае Хилла-Шрёдингера, - был использован Б. С. Митягиным (неопубликованная рукопись, лето 2000 г.) при доказательстве плотности конечнозонных потенциалов и в [55] при модифицированном изложении результатов из [37]-[39]. Теперь тот же прием помогает упростить доказательство нашей теоремы 58, данное в [19].

\section{4. Несамосопряженный случай}

4.1. Предварительные леммы. Мы знаем из результатов о локализации спектров (раздел 1), что при больших $n$ (или $|n|)$ оператор Хилла-Шрёдингера (или Дирака) $L=L^{0}+V$ имеет в круге с центром $n^{2}$ (или $n$ ) и радиусом $r=r(v)$ два (периодических, если $n$ четно, и антипериодических, если $n$ нечетно) собственных значения с учетом их алгебраической кратности. Напомним, что мы обозначаем через $E=E_{n}$ и $E^{0}=E_{n}^{0}$ соответствующие двумерные инвариантные подпространства операторов $L$ и $L^{0}$, а через $P=P_{n}$ и $P^{0}=P_{n}^{0}-$ проекторы Коши-Рисса на $E_{n}$ и $E_{n}^{0}$ соответственно. По предложениям 11 и 19, мы имеем:

$$
\left\|P_{n}-P_{n}^{0}: L^{2} \rightarrow L^{2}\right\| \leqslant\left\|P_{n}-P_{n}^{0}: L^{2} \rightarrow L^{\infty}\right\| \leqslant \kappa_{n} \rightarrow 0 .
$$

Теперь мы фиксируем $n \in \mathbb{N}$ (или $n \in \mathbb{Z}$ ) и рассматриваем соответствующие объекты такие, как $E=E_{n}, P=P_{n}$ и т. д., опуская индекс $n$ в обозначениях. Подпространство $E^{0}=E_{n}^{0}$ имеет следующий стандартный базис из собственных векторов $L^{0}$ (соответствующих собственному значению $\lambda^{0}=\lambda_{n}^{0}$ оператора $L^{0}$, где $\lambda_{n}^{0}=n^{2}$ или $\left.n\right)$ :

$$
e^{1}(x)=e^{-i n x}, \quad e^{2}(x)=e^{i n x}, \quad n \in \mathbb{N},
$$

в случае Хилла-Шрёдингера и

$$
e^{1}(x)=\left(\begin{array}{c}
e^{-i n x} \\
0
\end{array}\right), \quad e^{2}(x)=\left(\begin{array}{c}
0 \\
e^{i n x}
\end{array}\right), \quad n \in \mathbb{Z},
$$

в случае Дирака.

Если ограничение $L$ на $E$ имеет два собственных значения, то мы обозначаем их через $\lambda^{+}$и $\lambda^{-}$, где $\lambda^{+}-$собственное значение с большей вещественной частью, или с большей мнимой частью, если их вещественные части равны, и полагаем: $\gamma=\lambda^{+}-\lambda^{-}$.

Следующая лемма - своего рода уточнение и усиление леммы 21. Она выбирает специальный ортонормированный базис в $E$; этот базис играет критическую роль в построениях этой секции.

ЛЕмма 59. В предположениях и обозначениях, сделанных выше, если $|n|$ достаточно велико, то существует пара векторов $f, \varphi \in E=E_{n}$ такая, что

(a) $\|f\|=1,\|\varphi\|=1,\langle f, \varphi\rangle=0$;

(b) $L f=\lambda^{+} f$; 
(c) $L \varphi=\lambda^{+} \varphi-\gamma \varphi+\xi f$.

Более того, если $\varphi^{0}=P^{0} \varphi$, мы имеем:

$$
|\xi| \leqslant 4|\gamma|+2\left\|\left(z^{+}-S\left(\lambda^{+}\right)\right) \varphi^{0}\right\|
$$

$u$

$$
\left\|\left(z^{+}-S\left(\lambda^{+}\right)\right) \varphi^{0}\right\| \leqslant 2(|\xi|+|\gamma|),
$$

где $S\left(\lambda^{+}\right): E^{0} \rightarrow E^{0}-$ оператор, построенный в лемме $21, z^{+}=\lambda^{+}-n^{2}$ в случае Хилла-Шрёдингера и $z^{+}=\lambda^{+}-n$ в случае Дирака.

ДоказАтЕЛЬСтво. Пусть $f \in E$ такой единичный вектор, что $L f=\lambda^{+} f$. Выберем единичный вектор $\varphi$, ортогональный к $f$. Тогда (a) и (b) выполнены.

Если $\gamma=\lambda^{+}-\lambda^{-} \neq 0$, то существует второй собственный вектор $h \in E$ такой, что

$$
h=a f+b \varphi, \quad b \neq 0, \quad L h=\lambda^{-} h=\left(\lambda^{+}-\gamma\right) h .
$$

Поэтому

$$
a \lambda^{+} f+b L \varphi=\left(\lambda^{+}-\gamma\right)(a f+b \varphi),
$$

и, выбирая $\xi=-a \gamma / b$, мы приходим к (c).

Если $\lambda^{+}$имеет геометрическую кратность 2 , то $\gamma=0, \xi=0$ и (с) выполнено в форме $L \varphi=\lambda^{+} \varphi$.

Если же $\lambda^{+}$имеет алгебраическую кратность 2 , но геометрическую кратность 1 (жорданов блок), то $\gamma=0$ и $L \varphi=\lambda^{+} \varphi+\xi f$, так что (с) выполнено с некоторым $\xi \neq 0$.

Перепишем (c) в форме

$$
\left(\lambda^{+}-L\right) \varphi=\gamma \varphi-\xi f
$$

(ср. с (2.6)). В доказательстве леммы 21 было показано, что из (2.6) вытекает (2.13), и, следовательно, (с) влечет за собой:

$$
\left(z^{+}-S_{\lambda^{+}}\right) \varphi^{0}=-\xi f^{0}+\gamma \varphi^{0}+P^{0}(1-T)^{-1} T\left[-\xi\left(f-f^{0}\right)+\gamma\left(\varphi-\varphi^{0}\right)\right],
$$

где

$$
f^{0}=P^{0} f, \quad \varphi^{0}=P^{0} \varphi, \quad f-f^{0}=\left(P-P^{0}\right) f, \quad \varphi-\varphi^{0}=\left(P-P^{0}\right) \varphi,
$$

а оператор $T$ определен в лемме 21. В случае Хилла-Шрёдингера $\|T\|=$ $\left\|T_{n}\right\| \rightarrow 0$ (по лемме 23) и, следовательно, $\left\|(1-T)^{-1} T\right\| \leqslant 1$ для достаточно больших $n \in \mathbb{N}$. В случае Дирака $\left\|T^{2}\right\|=\left\|\left(T_{n}\right)^{2}\right\| \rightarrow 0$ (по лемме 33) и, в силу (2.121), мы имеем, что $\|T\| \leqslant C_{1}(\|v\|)$. Таким образом, для достаточно больших $|n|$ мы имеем:

$$
\left\|(1-T)^{-1} T\right\| \leqslant C,
$$

где $C=2 C_{1}(\|v\|)$.

Перепишем теперь (4.6) как

$$
\xi\left(f^{0}+f_{3}\right)=\gamma\left(\varphi^{0}+\varphi_{3}\right)-\left(z^{+}-S_{\lambda^{+}}\right) \varphi^{0},
$$

где

$$
f_{3}=P^{0}(1-T)^{-1} T\left(f-f^{0}\right), \quad \varphi_{3}=P^{0}(1-T)^{-1} T\left(\varphi-\varphi^{0}\right) .
$$


В силу (4.1), мы имеем:

$$
\left\|f-f^{0}\right\|=\left\|\left(P-P^{0}\right) f\right\| \leqslant \kappa .
$$

Следовательно, учитывая, что $f-f^{0}=\left(1-P^{0}\right) f$ ортогонален к $f^{0}$, мы получаем:

$$
1=\|f\|^{2}=\left\|f^{0}\right\|^{2}+\left\|f-f^{0}\right\|^{2} \leqslant\left\|f^{0}\right\|^{2}+\kappa^{2} .
$$

Таким образом,

$$
\left\|f-f^{0}\right\| \leqslant \kappa, \quad\left\|f^{0}\right\|^{2} \geqslant 1-\kappa^{2} .
$$

Конечно, такое же рассуждение показывает, что

$$
\left\|\varphi-\varphi^{0}\right\| \leqslant \kappa, \quad\left\|\varphi^{0}\right\|^{2} \geqslant 1-\kappa^{2} .
$$

Теперь, в силу (4.8), (4.10) и (4.11), мы имеем:

$$
\left\|f_{3}\right\| \leqslant\left\|(1-T)^{-1} T\right\| \cdot\left\|f-f^{0}\right\| \leqslant C \kappa, \quad\left\|\varphi_{3}\right\| \leqslant\left\|(1-T)^{-1} T\right\| \cdot\left\|\varphi-\varphi^{0}\right\| \leqslant C \kappa .
$$

Следовательно,

$$
1-\kappa-C \kappa \leqslant\left\|f^{0}\right\|-\left\|f_{3}\right\| \leqslant\left\|f^{0}+f_{3}\right\| \leqslant\left\|f^{0}\right\|+\left\|f_{3}\right\| \leqslant 1+C \kappa,
$$

и, по тем же самым рассуждениям, $1-(C+1) \kappa \leqslant\left\|\varphi^{0}+\varphi_{3}\right\| \leqslant 1+C \kappa$. Таким образом, для достаточно больших $|n|$ (так как $\kappa=\kappa_{n} \rightarrow 0$ ) мы получаем:

$$
1 / 2 \leqslant\left\|f^{0}+f_{3}\right\| \leqslant 2, \quad 1 / 2 \leqslant\left\|\varphi^{0}+\varphi_{3}\right\| \leqslant 2 .
$$

Теперь, по неравенству треугольника, (4.9) влечет за собой (4.4) и (4.5). Лемма 59 доказана.

Далее мы используем и лемму 59, и ее обозначения. Пусть $f, \varphi \in E$ - ортогональная пара векторов, построенных в лемме 59 , а $f_{1}^{0}, f_{2}^{0}$ и $\varphi_{1}^{0}, \varphi_{1}^{0}$ - координаты векторов $f^{0}=P^{0} f$ и $\varphi^{0}=P^{0} \varphi$ относительно базиса $\left\{e^{1}, e^{2}\right\}$, т.е.

$$
f^{0}(x)=f_{1}^{0} e^{1}(x)+f_{2}^{0} e^{2}(x), \quad \varphi^{0}(x)=\varphi_{1}^{0} e^{1}(x)+\varphi_{2}^{0} e^{2}(x) .
$$

Тогда мы имеем: $L f=\lambda^{+} f$, и, по лемме 21, вектор $f^{0}=P^{0} f$ - собственный вектор оператора $L^{0}+S\left(\lambda^{+}\right): E^{0} \rightarrow E^{0}$ с собственным значением $\lambda^{+}$, что приводит нас к соотношениям

$$
\left(\begin{array}{cc}
\zeta^{+} & B^{-} \\
B^{+} & \zeta^{+}
\end{array}\right)\left(\begin{array}{l}
f_{1}^{0} \\
f_{2}^{0}
\end{array}\right)=\left(\begin{array}{c}
\zeta^{+} f_{1}^{0}+B^{-} f_{2}^{0} \\
B^{+} f_{1}^{0}+\zeta^{+} f_{2}^{0}
\end{array}\right)=\left(\begin{array}{l}
0 \\
0
\end{array}\right), \quad\left(\zeta^{+}\right)^{2}=B^{+} B^{-},
$$

где $\left(\begin{array}{cc}\zeta^{+} & B^{-} \\ B^{+} & \zeta^{+}\end{array}\right)$- матричное представление оператора $z^{+}-S\left(\lambda^{+}\right)$, т.е., ввиду (2.33) и (2.80),

$$
\zeta^{+}=z^{+}-\alpha_{n}\left(z^{+}\right), \quad B^{ \pm}=\beta_{n}^{ \pm}\left(z^{+}\right), \quad z^{+}=\lambda^{+}-\lambda^{0} .
$$

Лемма 60. С введенными выше обозначениями, при достаточно больших $|n|$ ми имеем:

$$
\frac{1}{2}\left(\left|B^{+}\right|+\left|B^{-}\right|\right) \leqslant\left\|\left(z^{+}-S\left(\lambda^{+}\right)\right) \varphi^{0}\right\| \leqslant\left(\left|B^{+}\right|+\left|B^{-}\right|\right) .
$$


ДоказатеЛЬСтво. Вектор

$$
\psi^{0}=-\overline{f_{2}^{0}} e_{1}+\overline{f_{1}^{0}} e_{2}
$$

ортогонален к $f^{0}$, и $\left\|\psi^{0}\right\|=\left\|f^{0}\right\|$. Следовательно, вектор $\varphi^{0}$ есть линейная комбинация $f^{0}$ и $\psi^{0}$, так что мы имеем:

$$
\varphi^{0}=t f^{0}+s \psi^{0},
$$

и, в силу (4.13), мы получаем:

$$
\left(z^{+}-S\left(\lambda^{+}\right)\right) \varphi^{0}=s \cdot\left(z^{+}-S\left(\lambda^{+}\right)\right) \psi^{0} .
$$

Ввиду (4.12) и (4.13), векторы $\overline{\zeta^{+}} e^{1}+\overline{B^{-}} e^{2}$ и $\overline{B^{+}} e^{1}+\overline{\zeta^{+}} e^{2}$ ортогональны к $f^{0}$ и поэтому пропорциональны вектору $\psi^{0}$. Таким образом,

$$
\begin{aligned}
\left|\zeta^{+} \psi_{1}^{0}+B^{-} \psi_{2}^{0}\right|^{2} & =\left|\left\langle\psi^{0}, \overline{\zeta^{+}} e^{1}+\overline{B^{-}} e^{2}\right\rangle\right|^{2} \\
& =\left\|\psi^{0}\right\|^{2}\left\|\overline{\zeta^{+}} e^{1}+\overline{B^{-}} e^{2}\right\|^{2}=\left\|\psi^{0}\right\|^{2}\left(\left|\zeta^{+}\right|^{2}+\left|B^{-}\right|^{2}\right)
\end{aligned}
$$

и по тем же рассуждениям

$$
\left|\psi_{1}^{0} B^{+}+\psi_{2}^{0} \zeta^{+}\right|^{2}=\left\|\psi^{0}\right\|^{2}\left(\left|B^{+}\right|^{2}+\left|\zeta^{+}\right|^{2}\right) .
$$

В силу (4.13), мы знаем, что $\left(\zeta^{+}\right)^{2}=B^{+} B^{-}$. Поэтому (4.18) и (4.19) влекут за собой:

$\left\|\left(z^{+}-S\left(\lambda^{+}\right)\right) \psi^{0}\right\|^{2}=\left(\left|\zeta^{+} \psi_{1}^{0}+B^{-} \psi_{2}^{0}\right|^{2}+\left|\psi_{1}^{0} B^{+}+\psi_{2}^{0} \zeta^{+}\right|^{2}\right)=\left\|\psi^{0}\right\|^{2}\left(\left|B^{+}\right|+\left|B^{-}\right|\right)^{2}$, так что, ввиду (4.17), мы имеем:

$$
\left\|\left(z^{+}-S\left(\lambda^{+}\right)\right) \varphi^{0}\right\|=|s| \cdot\left\|\psi^{0}\right\|\left(\left|B^{+}\right|+\left|B^{-}\right|\right) .
$$

В силу (4.12), векторы $f-f^{0}$ и $\varphi-\varphi^{0}$ ортогональны к подпространству $E^{0}$, так что

$$
0=\langle f, \varphi\rangle=\left\langle f^{0}, \varphi^{0}\right\rangle+\left\langle f-f^{0}, \varphi-\varphi^{0}\right\rangle .
$$

Но тогда, в силу (4.10) и (4.11), мы имеем:

$$
\left|\left\langle f^{0}, \varphi^{0}\right\rangle\right|=\left|\left\langle f-f^{0}, \varphi-\varphi^{0}\right\rangle\right| \leqslant\left\|f-f^{0}\right\| \cdot\left\|\varphi-\varphi^{0}\right\| \leqslant \kappa^{2} .
$$

Учитывая, что $\left\|f^{0}\right\|^{2} \geqslant 1-\kappa^{2}$ (в силу (4.10)), мы оцениваем $|t|$ сверху:

$$
|t|=\frac{\left|\left\langle\varphi^{0}, f^{0}\right\rangle\right|}{\left\|f^{0}\right\|^{2}} \leqslant \frac{\kappa^{2}}{1-\kappa^{2}} \leqslant \kappa, \quad \text { если } \quad \kappa<1 / 2 .
$$

С другой стороны, в силу (4.16) и (4.10), мы имеем:

$$
1-\kappa^{2} \leqslant\left\|\varphi^{0}\right\|^{2}=|t|^{2}\left\|f^{0}\right\|^{2}+|s|^{2}\left\|\psi^{0}\right\|^{2} \leqslant \kappa^{2}+|s|^{2}\left\|\psi^{0}\right\|^{2},
$$

и, следовательно, если $\kappa<1 / 2$, то

$$
1 / 2<1-\kappa \leqslant|s| \cdot\left\|\psi^{0}\right\| \leqslant 1 .
$$

Очевидно, (4.20) и (4.22) влекут за собой (4.15). Это завершает доказательство леммы 60 . 
4.2. Оценки уклонений $\left|\mu-\lambda^{+}\right|$сверху. Далее мы используем те же обозначения, что и в предыдущем пункте. Как мы знаем из результатов о локализации спектров, оператор $L$ с граничными условиями Дирихле при достаточно больших $n \in \mathbb{N}$ в случае Хилла-Шрёдингера (или при достаточно больших $|n|, n \in \mathbb{Z}$, в случае Дирака) имеет собственное значение $\mu=\mu_{n}$, близкое к $\lambda^{+}=\lambda_{n}^{+}$. Теперь мы оценим уклонение $\left|\mu-\lambda^{+}\right|$в терминах параметров $\left|B^{-}\right|$и $\left|B^{+}\right|$.

Мы рассматриваем оба случая одновременно. В силу предложений 12 и 20, любая функция $h \in E$ непрерывна. Положим

$$
\begin{aligned}
& \ell_{0}(h)= \begin{cases}h(0) & \text { в случае Хилла-Шрёдингера, } \\
h_{1}(0)-h_{2}(0) & \text { в случае Дирака, }\end{cases} \\
& \ell_{1}(h)= \begin{cases}h(\pi) & \text { в случае Хилла-Шрёдингера, } \\
h_{1}(\pi)-h_{2}(\pi) & \text { в случае Дирака, }\end{cases}
\end{aligned}
$$

где $h(x)$ - комплекснозначная функция в случае Хилла-Шрёдингера и $h(x)=\left(\begin{array}{l}h_{1}(x) \\ h_{2}(x)\end{array}\right)$ в случае Дирака. Теперь $h$ удовлетворяет граничным условиям Дирихле тогда и только тогда, когда

$$
\ell_{0}(h)=0, \quad \ell_{1}(h)=0 .
$$

Мы построим специальную функцию $G \in E=E_{n}$, удовлетворяющую граничным условиям Дирихле, и воспользуемся ею, чтобы оценить уклонение $\left|\mu-\lambda^{+}\right|$.

Пусть $g$ - единичный собственный вектор Дирихле, соответствующий $\mu$, т.е.

$$
L_{\text {dir }} g=\mu g, \quad\|g\|=1,
$$

и пусть $P_{\text {dir }}$ - проектор Коши-Рисса на соответствующее одномерное подпространство.

ЛЕмма 61. В предположениях леммы 59

(а) существует вектор $G \in E=E_{n}$ вида

$$
G=a f+b \varphi, \quad\|G\|^{2}=|a|^{2}+|b|^{2}=1,
$$

maкой, что

$$
\ell_{0}(G)=0, \quad \ell_{1}(G)=0,
$$

m.е. $G$ лежит в области определения оператора $L_{\mathrm{dir}}$;

(b) ми имеем:

$$
\tau\left(\mu-\lambda^{+}\right)=b \xi\left\langle P_{\operatorname{dir}} f, g\right\rangle-b \gamma\left\langle P_{\operatorname{dir}} \varphi, g\right\rangle,
$$

əде $\tau=\tau_{n}$,

$$
1 / 2 \leqslant|\tau| \leqslant 2
$$

ДоказАтельство. (а) В силу (4.4) и (4.5), если $\ell_{0}(f)=0$, то $\ell_{1}(f)=$ $\pm \ell_{0}(f)=0$, поскольку $f-$ или периодический, или антипериодический собственный вектор. Таким образом, $G:=f$ имеет нужные свойства (4.26) и (4.27). 
Если $\ell_{0}(f) \neq 0$, то $\widetilde{G}=-\ell_{0}(f) \varphi(x)+\ell_{0}(\varphi) f(x)$ удовлетворяет условию (4.27), поскольку обе функции $f, \varphi$ одновременно или периодические, или антипериодические. Очевидно, нормированная функция $G=\widetilde{G} /\|\widetilde{G}\|$ удовлетворяет соотношению (4.26); это завершает доказательство части (a).

(b) Функция $G$, построенная в части (a), может быть записана как

$$
G=P_{\mathrm{dir}} G+\left(1-P_{\mathrm{dir}}\right) G=\tau g+u
$$

так что

$$
L_{\mathrm{dir}} G=\tau \mu g+L_{\mathrm{dir}} u .
$$

С другой стороны, по лемме 59,

$$
\begin{aligned}
L_{\mathrm{dir}} G & =L G=a L f+b L \varphi=a \lambda^{+} f+b\left(\lambda^{+} \varphi-\gamma \varphi+\xi f\right) \\
& =\lambda^{+}(a f+b \varphi)+b(\xi f-\gamma \varphi)=\lambda^{+} G+b(\xi f-\gamma \varphi),
\end{aligned}
$$

и тогда, в силу (4.30) и (4.31),

$$
\tau \mu g+L_{\operatorname{dir}} u=\lambda^{+}(\tau g+u)+b(\xi f-\gamma \varphi) .
$$

Применяя $P_{\text {dir }}$ к обеим сторонам $(4.32)$, мы получаем

$$
\tau\left(\mu-\lambda^{+}\right) g=b\left(\xi P_{\operatorname{dir}} f-\gamma P_{\operatorname{dir}} \varphi\right),
$$

откуда вытекает (4.28).

В силу (4.30) представим $\tau$ в виде суммы двух слагаемых:

$$
\tau=\left\langle P_{\mathrm{dir}}^{0} G, g\right\rangle+\left\langle\left(P_{\mathrm{dir}}-P_{\mathrm{dir}}^{0}\right) G, g\right\rangle .
$$

Ввиду предложений 11 и 19,

$$
\left|\left\langle\left(P_{\mathrm{dir}}-P_{\mathrm{dir}}^{0}\right) G, g\right\rangle\right| \leqslant\left\|\left(P_{\mathrm{dir}}-P_{\mathrm{dir}}^{0}\right) G\right\| \cdot\|g\| \leqslant \kappa .
$$

Чтобы оценить первое слагаемое, воспользуемся тем, что $P^{0}$ и $P_{\mathrm{dir}}^{0}-$ ортогональные проекторы. Положим

$$
G^{0}=P^{0} G, \quad g^{0}=P_{\mathrm{dir}}^{0} g .
$$

Тогда

$$
\left\langle P_{\mathrm{dir}}^{0} G, g\right\rangle=\left\langle G, P_{\mathrm{dir}}^{0} g\right\rangle=\left\langle P^{0} G, P_{\mathrm{dir}}^{0} g\right\rangle=\left\langle G^{0}, g^{0}\right\rangle,
$$

так как $P_{\mathrm{dir}}^{0} \in E^{0}$, а вектор $G-P^{0} G$ ортогонален подпространству $E^{0}$. Поэтому, в силу оценки для второго слагаемого, получаем

$$
\left|\left\langle G^{0}, g^{0}\right\rangle\right|-\kappa \leqslant|\tau| \leqslant\left|\left\langle G^{0}, g^{0}\right\rangle\right|+\kappa .
$$

Теперь остается оценить $\left\langle G^{0}, g^{0}\right\rangle$. Так как $G \in E$, то $G-G^{0}=\left(P-P^{0}\right) G$. Следовательно, в силу (4.1),

$$
\left\|G-G^{0}\right\| \leqslant\left\|G-G^{0}\right\|_{L^{\infty}} \leqslant\left\|P-P^{0}: L^{2} \rightarrow L^{\infty}\right\| \leqslant \kappa .
$$


Более того, так как $G-G^{0}$ ортогонален к $G^{0}$, то

$$
\left\|G^{0}\right\|^{2}=1-\left\|G-G^{0}\right\|^{2} \geqslant 1-\kappa^{2} .
$$

Напомним, что одномерное подпространство $E_{\text {dir }}^{0}$ порождено функцией

$$
s(x)= \begin{cases}\frac{1}{i \sqrt{2}}\left(e^{2}(x)-e^{1}(x)\right) & \text { в случае Хилла-Шрёдингера, } \\ \frac{1}{\sqrt{2}}\left(e^{2}(x)+e^{1}(x)\right) & \text { в случае Дирака. }\end{cases}
$$

Так как $\|s\|=1$, мы имеем:

$$
g^{0}(x)=\left\|g^{0}\right\| \cdot s(x)
$$

Более того, по предложениям 11 и 19,

$$
\left\|P_{\mathrm{dir}}-P_{\mathrm{dir}}^{0}: L^{2} \rightarrow L^{2}\right\| \leqslant\left\|P_{\mathrm{dir}}-P_{\mathrm{dir}}^{0}: L^{2} \rightarrow L^{\infty}\right\| \leqslant \kappa,
$$

где $\kappa=\kappa_{n} \rightarrow 0$. Следовательно, в силу (4.25),

$$
\left\|g-g^{0}\right\|=\left\|\left(P_{\text {dir }}-P_{\text {dir }}^{0}\right) g\right\| \leqslant \kappa, \quad\left\|g^{0}\right\|^{2}=1-\left\|g-g^{0}\right\|^{2} \geqslant 1-\kappa^{2} .
$$

С другой стороны, $\ell_{0}(G)=0$ в силу (4.27). Следовательно, в силу (4.1) и (4.23), мы имеем:

$$
\left|\ell_{0}\left(G^{0}\right)\right|=\left|\ell_{0}\left(G-G^{0}\right)\right| \leqslant 2\left\|G-G^{0}\right\|_{L^{\infty}}=2\left\|\left(P-P^{0}\right) G\right\|_{L^{\infty}} \leqslant 2 \kappa .
$$

Пусть $G_{1}^{0}$ и $G_{2}^{0}$ будут координатами вектора $G^{0}$ относительно канонического базиса $\left\{e^{1}, e^{2}\right\}$ в $E^{0}$, т.е. $G^{0}(x)=G_{1}^{0} e^{1}(x)+G_{2}^{0} e^{2}(x)$. Тогда, ввиду (4.1), (4.2) и $(4.23)$, мы имеем:

$$
\ell_{0}\left(G^{0}\right)= \begin{cases}G_{1}^{0}+G_{2}^{0} & \text { в случае Хилла-Шрёдингера } \\ G_{1}^{0}-G_{2}^{0} & \text { в случае Дирака. }\end{cases}
$$

Следовательно, в силу (4.39), мы имеем:

$$
\left|\ell_{0}\left(G^{0}\right)\right|=\left|G_{1}^{0} \pm G_{2}^{0}\right| \leqslant 2 \kappa
$$

что приводит нас к неравенству

$$
\left|G_{1}^{0}\right| \leqslant\left|G_{2}^{0}\right|+2 \kappa
$$

Отсюда, ввиду (4.35), вытекает, что

$$
1-\kappa^{2} \leqslant\left\|G^{0}\right\|^{2}=\left|G_{1}^{0}\right|^{2}+\left|G_{2}^{0}\right|^{2} \leqslant 2\left(\left|G_{2}^{0}\right|+2 \kappa\right)^{2},
$$

и тогда $1-\kappa \leqslant \sqrt{2}\left(\left|G_{2}^{0}\right|+2 \kappa\right) \leqslant \sqrt{2}\left|G_{2}^{0}\right|+3 \kappa$. Таким образом,

$$
\left|G_{2}^{0}\right| \geqslant \frac{1}{\sqrt{2}}(1-4 \kappa) \text {. }
$$


В случае Хилла-Шрёдингера, используя (4.36), (4.37) и (4.40), мы получаем, что

$$
\left|\left\langle G^{0}, g^{0}\right\rangle\right|=\frac{\left\|g^{0}\right\|}{\sqrt{2}}\left|G_{2}^{0}-G_{1}^{0}\right|=\frac{\left\|g^{0}\right\|}{\sqrt{2}}\left|2 G_{2}^{0}-\ell_{0}\left(G^{0}\right)\right| \geqslant \frac{\left\|g^{0}\right\|}{\sqrt{2}}\left(2\left|G_{2}^{0}\right|-\left|\ell_{0}\left(G^{0}\right)\right|\right) .
$$

Такие же оценки для $\left|\left\langle G^{0}, g^{0}\right\rangle\right|$ можно получить в случае Дирака. Действительно, в силу $(4.36),(4.37)$ и (4.40), мы имеем:

$$
\left|\left\langle G^{0}, g^{0}\right\rangle\right|=\frac{\left\|g^{0}\right\|}{\sqrt{2}}\left|G_{2}^{0}+G_{1}^{0}\right|=\frac{\left\|g^{0}\right\|}{\sqrt{2}}\left|2 G_{2}^{0}+\ell_{0}\left(G^{0}\right)\right| \geqslant \frac{\left\|g^{0}\right\|}{\sqrt{2}}\left(2\left|G_{2}^{0}\right|-\left|\ell_{0}\left(G^{0}\right)\right|\right) .
$$

Ввиду (4.33), (4.38), (4.39) и (4.41)-(4.42), это приводит нас к соотношению

$$
1+\kappa \geqslant|\tau| \geqslant \frac{1-\kappa^{2}}{\sqrt{2}}(\sqrt{2}(1-4 \kappa)-2 \kappa)-\kappa \geqslant 1-8 \kappa .
$$

Так как $\kappa=\kappa_{n} \rightarrow 0$, последняя оценка влечет за собой (4.29). Это завершает доказательство леммы 61.

ПРЕДЛОЖЕНИЕ 62. В обозначениях и предположениях $n .4 .1$ для достаточно больиих $|n|$ мьи имеем:

$$
\left|\mu-\lambda^{+}\right| \leqslant 18|\gamma|+8\left(\left|B^{+}\right|+\left|B^{-}\right|\right) .
$$

ДокАЗАТЕЛЬСтво. В силу (4.26), (4.28) и (4.29), для достаточно больших $|n|$ выполнено следующее неравенство:

$$
\left|\mu-\lambda^{+}\right| \leqslant 2|\xi|\left|\left\langle P_{\operatorname{dir}} f, g\right\rangle\right|+2|\gamma|\left|\left\langle P_{\operatorname{dir}} f, g\right\rangle\right| .
$$

Ввиду предложений 11 и 19 , мы имеем $\left\|P_{\text {dir }}-P_{\text {dir }}^{0}\right\| \leqslant \kappa$, где $\kappa=\kappa_{n} \rightarrow 0$. Так как $f, g$ - единичные векторы, а $P_{\mathrm{dir}}^{0}$ - ортогональный проектор, то

$$
\left|\left\langle P_{\text {dir }} f, g\right\rangle\right| \leqslant\left|\left\langle P_{\text {dir }}^{0} f, g\right\rangle\right|+\left|\left\langle\left(P_{\text {dir }}-P_{\text {dir }}^{0}\right) f, g\right\rangle\right| \leqslant 1+\kappa \leqslant 2
$$

для достаточно больших $|n|$. Тем же путем мы получаем, что

$$
\left|\left\langle P_{\operatorname{dir}} \varphi, g\right\rangle\right| \leqslant 1+\kappa \leqslant 2
$$

для достаточно больших $|n|$. Следовательно,

$$
\left|\mu-\lambda^{+}\right| \leqslant 4|\xi|+4|\gamma|
$$

для достаточно больших $|n|$.

С другой стороны, (4.4) из леммы 59 и (4.15) из леммы 60 приводят нас к неравенству

$$
|\xi| \leqslant 4|\gamma|+2\left(\left|B^{+}\right|+\left|B^{-}\right|\right)
$$

Эти две оценки влекут за собою (4.43). Предложение 62 доказано. 
4.3. Оценки уклонения $|\gamma|+\left|\mu-\lambda^{+}\right|$снизу. Оценки "лакун" $|\gamma|$ снизу доказаны в лемме 49. Это приводит к следующему утверждению.

ПРЕДЛОЖЕНИЕ 63. Для достаточно болъиих $|n|$ если

$$
\frac{1}{4}\left|B^{-}\right| \leqslant\left|B^{+}\right| \leqslant 4\left|B^{-}\right|
$$

mo

$$
\left|\beta_{n}^{-}\left(z_{n}^{*}\right)\right|+\left|\beta_{n}^{+}\left(z_{n}^{*}\right)\right| \leqslant 2\left|\gamma_{n}\right|,
$$

где $B^{ \pm}=\beta_{n}^{ \pm}\left(z^{+}\right)$и $z_{n}^{*}=\left(\lambda_{n}^{+}+\lambda_{n}^{-}\right) / 2-\lambda_{n}^{0}$ в случае простых собственных значений и $z_{n}^{*}=\lambda_{n}^{+}-\lambda_{n}^{0}$ в противном случае.

ДокАЗАТЕЛЬСтво. Если (4.45) выполнено, то или $B^{+}=B^{-}=0$, или $B^{+} B^{-} \neq 0$. Если $B^{+}=0$ и $B^{-}=0$, то $\lambda^{+}-$собственное значение геометрической кратности 2 оператора $P^{0} L^{0} P^{0}+S\left(\lambda^{+}\right)$. Это может случиться, в силу замечания 22 , тогда и только тогда, когда $\lambda^{+}$есть также собственное значение геометрической кратности 2 оператора $L$. Но тогда $\gamma_{n}=0, z_{n}^{*}=z_{n}^{+}$, и, следовательно, (4.46) выполнено.

Если $B^{+} B^{-} \neq 0$ и $\gamma_{n} \neq 0$, то утверждение следует из леммы 49 , поскольку (4.45) влечет за собой $t=\left|B^{+}\right| /\left|B^{-}\right| \in[1 / 4,4]$, и, следовательно, $2 \sqrt{t} /(1+t) \geqslant$ $4 / 5$.

Наконец, рассмотрим тот случай, когда $B^{+} B^{-} \neq 0$, но $\gamma_{n}=0$. По результатам о локализации спектров (теорема 9 в случае Хилла-Шрёдингера или теорема 17 в случае Дирака), существует такой круг $D=\{z:|z|<r\}$, что $\lambda=\lambda^{0}+z, z \in D$, есть собственное значение оператора $L$ тогда и только тогда, когда $z$ - корень основного уравнения

$$
h(z):=(\zeta(z))^{2}-\beta^{+}(z) \beta^{-}(z)=0, \quad \zeta(z)=z-\alpha(z) .
$$

Поэтому если $\gamma_{n}=0$, то $z^{+}=\lambda^{+}-\lambda^{0}$ оказывается единственным корнем уравнения $h(z)=0$ в круге $D$.

Более того, корень $z^{+}$имеет кратность 2. Действительно, рассмотрим два уравнения $z^{2}=0$ и $h(z)=0$ на круге $D$. Ввиду предложения 28 , или предложения 35, максимальные значения параметров $\left|\alpha_{n}(z)\right|$ и $\left|\beta_{n}^{ \pm}(z)\right|$ в круге $\partial D=$ $\{z:|z|=r\}$ стремятся к нулю, если $|n| \rightarrow \infty$. Следовательно, для достаточно больших $|n|$ мы имеем:

$$
\sup _{\partial D}\left|h(z)-z^{2}\right|<\sup _{\partial D}\left|z^{2}\right|
$$

и из теоремы Руше вытекает, что $z^{+}-$двойной корень уравнения $h(z)=0$. Но тогда производная $h$ обращается в нуль в точке $z^{+}$, т.е.

$$
2 \zeta\left(z^{+}\right) \cdot\left(1-\frac{d \alpha}{d z}\left(z^{+}\right)\right)=\frac{d \beta^{+}}{d z}\left(z^{+}\right) \cdot \beta^{-}\left(z^{+}\right)+\beta^{+}\left(z^{+}\right) \cdot \frac{d \beta^{-}}{d z}\left(z^{+}\right) .
$$

По лемме 29 в случае Хилла-Шрёдингера и по предложению 35 в случае Дирака, для достаточно больших $|n|$ мы имеем:

$$
\left|\frac{d \alpha}{d z}\left(z^{+}\right)\right| \leqslant \frac{1}{5}, \quad\left|\frac{d \beta^{ \pm}}{d z}\left(z^{+}\right)\right| \leqslant \frac{1}{5} .
$$


Следовательно, по неравенству треугольника, мы получаем:

$$
2\left|\zeta^{+}\right|\left(1-\frac{1}{5}\right) \leqslant \frac{1}{5}\left(\left|B^{+}\right|+\left|B^{-}\right|\right),
$$

где $\left|\zeta^{+}\right|=\left|\zeta\left(z^{+}\right)\right|=\sqrt{\left|B^{+} B^{-}\right|}$. Но тогда, ввиду (4.45), последнее неравенство влечет за собой, что

$$
8 \leqslant \sqrt{\left|B^{+} / B^{-}\right|}+\sqrt{\left|B^{-} / B^{+}\right|} \leqslant 4
$$

но это приводит к противоречию. Доказательство закончено.

Теперь мы рассматриваем случай, дополнительный к (4.45), т.е.

$$
\text { (a) } 4\left|B^{+}\right|<\left|B^{-}\right| \quad \text { или } \quad \text { (b) } 4\left|B^{-}\right|<\left|B^{+}\right| \text {. }
$$

Начнем со следующего технического утверждения.

ЛЕмма 64. Если $|n|$ достаточно велико и (4.47) выполнено, то

$$
\frac{1}{4} \leqslant \frac{\left|\ell_{0}(f)\right|}{\left|\ell_{0}(\varphi)\right|} \leqslant 4 .
$$

ДокАзАтельство. Доказательство основано на том факте, что при достаточно больших $n$ подпространство $E=E_{n}$ "близко" к $E^{0}=E_{n}^{0}$ в том смысле, что $\kappa=\kappa_{n}$ в (4.1) "мало", и, следовательно, мы можем оценить $\ell_{0}(f)$ и $\ell_{0}(\varphi)$ в терминах $\ell_{0}\left(f^{0}\right)$ и $\ell_{0}\left(\varphi^{0}\right)$ соответственно. Далее мы предполагаем, что $|n|$ настолько велико, что $\kappa<1 / 4$; тогда $\kappa^{2}<\kappa / 4$. В силу (4.1) и (4.7), мы имеем:

$$
\left|\ell_{0}(f)-\ell_{0}\left(f^{0}\right)\right| \leqslant\left\|f-f^{0}\right\|_{L^{\infty}}=\left\|\left(P-P^{0}\right) f\right\|_{L^{\infty}} \leqslant \kappa, \quad\left|\ell_{0}(\varphi)-\ell_{0}\left(\varphi^{0}\right)\right| \leqslant \kappa .
$$

Мы рассмотрим только (a) в (4.47), поскольку случай (b) аналогичен и с ним можно справиться тем же способом.

Ввиду (4.13), мы имеем:

$$
\zeta^{+} f_{1}^{0}+B^{-} f_{2}^{0}=0, \quad\left(\zeta^{+}\right)^{2}=B^{-} B^{+},
$$

и, следовательно,

$$
\left|B^{-}\right|\left|f_{2}^{0}\right|=\left|\zeta^{+}\right|\left|f_{1}^{0}\right|=\left|B^{-} B^{+}\right|^{1 / 2}\left|f_{1}^{0}\right| .
$$

Тогда, в силу (а) в (4.47), мы имеем:

$$
\left|f_{2}^{0}\right|=\sqrt{\left|B^{+}\right| /\left|B^{-}\right|}\left|f_{1}^{0}\right| \leqslant \frac{1}{2}\left|f_{1}^{0}\right| .
$$

Так как $\left\|f^{0}\right\|=\left\|P^{0} f\right\| \leqslant 1$, то отсюда вытекает, что

$$
5\left|f_{2}^{0}\right|^{2} \leqslant\left|f_{1}^{0}\right|^{2}+\left|f_{2}^{0}\right|^{2} \leqslant 1,
$$

и это приводит к соотношению

$$
\left|f_{2}^{0}\right| \leqslant 1 / \sqrt{5}
$$


С другой стороны, (4.50) и (4.10) влекут за собой, что

$$
1-\kappa^{2} \leqslant\left\|f^{0}\right\|^{2}=\left|f_{1}^{0}\right|^{2}+\left|f_{2}^{0}\right|^{2} \leqslant(1+1 / 4)\left|f_{1}^{0}\right|^{2},
$$

и поэтому

$$
\left|f_{1}^{0}\right| \geqslant \frac{2}{\sqrt{5}} \sqrt{1-\kappa^{2}}>\frac{2}{\sqrt{5}}(1-\kappa) .
$$

Теперь мы выводим такие же оценки для $\varphi^{0}=P^{0} \varphi$. Ввиду (4.21), мы имеем:

$$
\left|\left\langle f^{0}, \varphi^{0}\right\rangle\right|=\left|f_{1}^{0} \varphi_{1}^{0}+f_{2}^{0} \varphi_{2}^{0}\right| \leqslant \kappa^{2} .
$$

По неравенству треугольника мы получаем:

$$
\left|f_{1}^{0} \varphi_{1}^{0}\right| \leqslant\left|f_{2}^{0} \varphi_{2}^{0}\right|+\kappa^{2} .
$$

Разделим обе части этого неравенства на $\left|f_{1}^{0}\right|$; ввиду (4.50), мы имеем:

$$
\left|\varphi_{1}^{0}\right| \leqslant \frac{\left|f_{2}^{0}\right|}{\left|f_{1}^{0}\right|}\left|\varphi_{2}^{0}\right|+\frac{\kappa^{2}}{\left|f_{1}^{0}\right|} \leqslant \frac{1}{2}\left|\varphi_{2}^{0}\right|+\kappa
$$

(Мы предполагаем, что $\kappa<1 / 4$, и, следовательно, из (4.52) вытекает неравенство $\left|f_{1}^{0}\right|>\kappa$.)

Соотношения (4.11) и (4.53) влекут за собой, что

$$
1-\kappa^{2} \leqslant\left|\varphi_{1}^{0}\right|^{2}+\left|\varphi_{2}^{0}\right|^{2} \leqslant(1+1 / 4)\left|\varphi_{2}^{0}\right|^{2}+\kappa+\kappa^{2}
$$

и так как $\kappa<1 / 4$, отсюда вытекает, что

$$
\left|\varphi_{2}^{0}\right| \geqslant \frac{2}{\sqrt{5}} \sqrt{1-2 \kappa}>\frac{2}{\sqrt{5}}(1-2 \kappa) .
$$

С другой стороны, в силу (4.53), мы имеем:

$$
1 \geqslant\left\|\varphi^{0}\right\|^{2}=\left|\varphi_{1}^{0}\right|^{2}+\left|\varphi_{2}^{0}\right|^{2} \geqslant\left|\varphi_{1}^{0}\right|^{2}+4\left(\left|\varphi_{1}^{0}\right|-\kappa\right)^{2} \geqslant 5\left(\left|\varphi_{1}^{0}\right|-\kappa\right)^{2},
$$

и, следовательно,

$$
\left|\varphi_{1}^{0}\right| \leqslant \frac{1}{\sqrt{5}}+\kappa
$$

Ввиду (4.2), (4.3) и (4.23), мы имеем:

$$
\ell_{0}(h)=\eta_{1} \pm \eta_{2}, \quad \text { если } \quad h(x)=\eta_{1} e_{1}(x)+\eta_{2} e_{2}(x) \in E^{0},
$$

и, следовательно,

$$
|| \eta_{1}|-| \eta_{2}|| \leqslant\left|\ell_{0}(h)\right| \leqslant\left|\eta_{1}\right|+\left|\eta_{2}\right| \leqslant \sqrt{2}\|h\| .
$$

Таким образом, ввиду (4.51), (4.52), (4.54) и (4.55), мы получаем:

$$
\frac{1}{\sqrt{5}}-\kappa \leqslant \ell_{0}(f) \leqslant \sqrt{2}, \quad \frac{1}{\sqrt{5}}-3 \kappa \leqslant \ell_{0}\left(\varphi^{0}\right) \leqslant \sqrt{2} .
$$


В силу (4.49), эти оценки приводят нас к неравенству

$$
\frac{1 / \sqrt{5}-2 \kappa}{\sqrt{2}+\kappa} \leqslant \frac{\left|\ell_{0}(f)\right|}{\left|\ell_{0}(\varphi)\right|} \leqslant \frac{\sqrt{2}+\kappa}{1 / \sqrt{5}-4 \kappa} .
$$

Если $\kappa \rightarrow 0$, то левая часть в (4.56) стремится к $1 / \sqrt{10}$, в то время как правая часть стремится к $\sqrt{10}$. Таким образом, если $\kappa=\kappa_{n}$ достаточно мало, т.е. если $|n|$ выбрано достаточно большим, то (4.48) выполнено. Лемма 64 доказана.

ПРЕДЛОЖЕНИЕ 65. Если (4.47) выполнено, то для достаточно больиих $|n|$ мы имеем:

$$
\left|B^{+}\right|+\left|B^{-}\right| \leqslant 36|\gamma|+144\left|\mu-\lambda^{+}\right| .
$$

ДокАЗАТЕЛЬСтво. Пусть $G=a f+b \varphi-$ построенная в лемме 61 функция с граничными условиями Дирихле. Тогда

$$
0=\ell_{0}(G)=a \ell_{0}(f)+b \ell_{0}(\varphi)
$$

и, следовательно, в силу (4.48) в лемме 64, мы имеем:

$$
1 / 4 \leqslant|b| /|a|=\left|\ell_{0}(f)\right| /\left|\ell_{0}(\varphi)\right| \leqslant 4 .
$$

Таким образом, $|a| \leqslant 4|b|$, и мы получаем:

$$
1=4|b|^{2}+|b|^{2}=17|b|^{2} \Rightarrow|b|>1 / \sqrt{17}>2 / 9 .
$$

С другой стороны, в силу (4.28) мы имеем:

$$
b \xi\left\langle P_{\operatorname{dir}} f, g\right\rangle=\tau\left(\mu-\lambda^{+}\right)+b \gamma\left\langle P_{\operatorname{dir}} \varphi, g\right\rangle,
$$

так что из неравенства треугольника вытекает:

$$
|b||\xi|\left|\left\langle P_{\operatorname{dir}} f, g\right\rangle\right| \leqslant|\tau|\left|\mu-\lambda^{+}\right|+|b||\gamma|\left|\left\langle P_{\operatorname{dir}} \varphi, g\right\rangle\right| .
$$

Тогда для достаточно больших $|n|$, учитывая, что $|b|>2 / 9,|\tau| \leqslant 2$ в силу (4.29) и $\left|\left\langle P_{\operatorname{dir}} \varphi, g\right\rangle\right| \leqslant 2$ в силу (4.44), мы получаем:

$$
|\xi|\left|\left\langle P_{\operatorname{dir}} f, g\right\rangle\right| \leqslant 9\left|\mu-\lambda^{+}\right|+2|\gamma| .
$$

Теперь мы оцениваем $\left|\left\langle P_{\operatorname{dir}} f, g\right\rangle\right|$ снизу. В силу предложений 11 и 19, мы имеем $\left|\left\langle\left(P_{\text {dir }}-P_{\text {dir }}^{0}\right) f, g\right\rangle\right| \leqslant \kappa$, где $\kappa=\kappa_{n} \rightarrow 0$. Следовательно, по неравенству треугольника мы получаем:

$$
\left|\left\langle P_{\text {dir }} f, g\right\rangle\right| \leqslant\left|\left\langle P_{\text {dir }}^{0} f, g\right\rangle\right|-\kappa .
$$

Пусть

$$
f^{0}=P^{0} f, \quad g^{0}=P_{\mathrm{dir}}^{0} g .
$$

Так как $P^{0}$ и $P_{\mathrm{dir}}^{0}$ - ортогональные проекторы, мы имеем

$$
\left\langle P_{\mathrm{dir}}^{0} f, g\right\rangle=\left\langle f, P_{\mathrm{dir}}^{0} g\right\rangle=\left\langle f^{0}, g^{0}\right\rangle,
$$


поскольку $g^{0} \in E^{0}$ и $f-f^{0}$ ортогонален подпространству $E^{0}$. Следовательно,

$$
\left|\left\langle P_{\operatorname{dir}} f, g\right\rangle\right| \geqslant\left|\left\langle f^{0}, g^{0}\right\rangle\right|-\kappa .
$$

Ввиду (4.36)-(4.37), мы имеем:

$$
\left|\left\langle f^{0}, g^{0}\right\rangle\right|=\left\|g^{0}\right\| \cdot\left|\left\langle f^{0}, s\right\rangle\right| \geqslant(1-\kappa) \frac{1}{\sqrt{2}}|| f_{1}^{0}|-| f_{2}^{0} \| .
$$

Если имеет место случай (а) в (4.47), то (4.51) и (4.52) из доказательства леммы 64 влекут за собой, что

$$
\left|\left\langle f^{0}, g^{0}\right\rangle\right| \geqslant(1-\kappa) \frac{1}{\sqrt{2}} \cdot \frac{1}{\sqrt{5}}(1-2 \kappa)>\frac{1}{\sqrt{10}}-\kappa .
$$

Если же (b) в (4.47) выполнено, то мы получаем такие же оценки. Таким образом, для достаточно малых $\kappa$ мы имеем:

$$
\left|\left\langle P_{\text {dir }} f, g\right\rangle\right| \geqslant \frac{1}{\sqrt{10}}-2 \kappa \geqslant \frac{1}{4} .
$$

Наконец, из (4.58) и (4.59) вытекает, что

$$
|\xi| \leqslant 8|\gamma|+36\left|\mu-\lambda^{+}\right|
$$

Это неравенство вместе с (4.5) и (4.15) влечет за собой (4.57). Это завершает доказательство предложения 65.

4.4. Основные результаты. Основным результатом этого пункта является следующая теорема.

Теорема 66. Пусть $L$ - периодический оператор Хилла-Шрёдингера или Дирака. Для достаточно больших $n$ если $\lambda_{n}^{+}, \lambda_{n}^{-}-n$-я пара периодических (для четных $n)$ или антипериодических (для нечетных $n$ ) собственных значений оператора $L, \gamma_{n}=\lambda_{n}^{+}-\lambda_{n}^{-}, a \mu_{n}-n$-е собственное значение Дирихле onepamopa $L$, mo

$$
\frac{1}{144}\left(\left|\beta_{n}^{-}\left(z_{n}^{*}\right)\right|+\left|\beta_{n}^{+}\left(z_{n}^{*}\right)\right|\right) \leqslant\left|\gamma_{n}\right|+\left|\mu_{n}-\lambda_{n}^{+}\right| \leqslant 54\left(\left|\beta_{n}^{-}\left(z_{n}^{*}\right)\right|+\left|\beta_{n}^{+}\left(z_{n}^{*}\right)\right|\right) .
$$

ДокАЗАТЕльство. По теоремам о локализации (теорема 9 в случае ХиллаШрёдингера или теорема 17 в случае Дирака) и по лемме 29 в случае ХиллаШрёдингера и предложению 35 в случае Дирака,

$$
\sup _{\left[z_{n}^{-}, z_{n}^{+}\right]}\left|\frac{\partial \alpha_{n}}{\partial z}\right| \leqslant \varepsilon_{n}, \quad \sup _{\left[z_{n}^{-}, z_{n}^{+}\right]}\left|\frac{\partial \beta_{n}^{ \pm}}{\partial z}\right| \leqslant \varepsilon_{n}, \quad \varepsilon_{n} \downarrow 0,
$$

где $\left[z_{n}^{-}, z_{n}^{+}\right]$обозначает отрезок с концевыми точками $z_{n}^{-}$и $z_{n}^{+}$. Тогда, поскольку $\left|z_{n}^{+}-z_{n}^{*}\right| \leqslant\left|\gamma_{n}\right|=\left|z_{n}^{+}-z_{n}^{-}\right|$, мы имеем:

$$
\left|\beta_{n}^{ \pm}\left(z_{n}^{+}\right)-\beta_{n}^{ \pm}\left(z_{n}^{*}\right)\right| \leqslant \varepsilon_{n}\left|\gamma_{n}\right| .
$$


Отсюда, по неравенству треугольника, для достаточно больших $|n|$ вытекает:

$$
\left|B^{ \pm}\right|-\left|\gamma_{n}\right| \leqslant\left|\beta_{n}^{ \pm}\left(z_{n}^{*}\right)\right| \leqslant\left|B^{ \pm}\right|+\left|\gamma_{n}\right|
$$

где (ср. с (4.14)) $B^{ \pm}=\left|\beta_{n}^{ \pm}\left(z_{n}^{+}\right)\right|$.

Ввиду (4.61), предложения 63 и 65 влекут за собой левое неравенство в (4.60). С другой стороны, для достаточно больших $|n|$ лемма 40 показывает, что

$$
\left|\gamma_{n}\right| \leqslant 2\left(\left|\beta_{n}^{-}\left(z_{n}^{*}\right)\right|+\left|\beta_{n}^{+}\left(z_{n}^{*}\right)\right|\right) .
$$

Следовательно, предложение 62 и неравенства (4.61) влекут за собой правое неравенство в (4.60). Теорема 66 доказана.

Следующая теорема суммирует наши результаты об операторах ХиллаШрёдингера с комплекснозначными потенциалами.

Теорема 67. Если $L=L^{0}+v(x)$ - оператор Хилла-Шрёдингера с периодическим потенциалом $v \in L^{2}([0, \pi])$, то для $n>n_{0}=n_{0}(\|v\|)$ оператор $L$ имеет в круге с иентром $n^{2}$ и радиусом $r=r(v)$ в точности два (с учетом их кратности) периодических (для четных $n$ ) или антипериодических (для нечетных $n$ ) собственных значений $\lambda_{n}^{+} u \lambda_{n}^{-}$и одно собственное значение Дирихле $\mu_{n}$.

$\Pi$ Путь

$$
\Delta_{n}=\left|\lambda_{n}^{+}-\lambda_{n}^{-}\right|+\left|\lambda_{n}^{+}-\mu_{n}\right|, \quad n>n_{0} ;
$$

тогда для любого субмультипликативного веса $\Omega$

$$
v \in H(\Omega) \Rightarrow\left(\Delta_{n}\right) \in \ell^{2}(\Omega) .
$$

Обратно, если $\Omega=(\Omega(n))_{n \in \mathbb{Z}}-$ такой субмультипликативный вес, что

$$
\frac{\log \Omega(n)}{n} \searrow 0, \quad \text { когда } n \rightarrow \infty,
$$

mo

$$
\left(\Delta_{n}\right) \in \ell^{2}(\Omega) \Rightarrow v \in H(\Omega) .
$$

Если $\Omega$ - субмультипликативный вес экспоненциального типа, т.е.

$$
\lim _{n \rightarrow \infty} \frac{\log \Omega(n)}{n}>0,
$$

mo

$$
\left(\Delta_{n}\right) \in \ell^{2}(\Omega) \Rightarrow \exists \varepsilon>0: \quad v \in H\left(e^{\varepsilon|n|}\right) .
$$

ДоказАтельство. Существование такого $n_{0}=n_{0}(\|v\|)$, что $\lambda_{n}^{ \pm}$и $\mu_{n}$ вполне определены для всех $n>n_{0}$, непосредственно вытекает из теоремы 9. Более того, без ограничения общности мы можем считать $n_{0}$ настолько большим, что теорема 66 выполняется, если $n>n_{0}$.

Пусть $\Omega$ - субмультипликативный вес и $v \in H(\Omega)$. По правому неравенству в (4.60) теоремы 66 , при $n>n_{0}$ мы имеем:

$\Delta_{n} \leqslant 54\left(\left|\beta_{n}^{-}\left(z_{n}^{*}\right)\right|+\left|\beta_{n}^{+}\left(z_{n}^{*}\right)\right|\right) \leqslant 54\left(\left|v_{-n}\right|+\left|v_{n}\right|+\left|\beta_{n}^{-}\left(z_{n}^{*}\right)-v_{-n}\right|+\left|\beta_{n}^{+}\left(z_{n}^{*}\right)-v_{n}\right|\right)$. 
Отсюда, ввиду (2.52), из предложения 28 вытекает, что

$$
\Delta_{n} \Omega(n) \leqslant 54\left(\left|v_{-n}\right| \Omega(n)+\left|v_{n}\right| \Omega(n)+\frac{8}{n}\|v\|_{\Omega}^{2}\right) .
$$

Таким образом, мы получаем:

$$
\sum_{n>n_{0}}\left|\Delta_{n}\right|^{2}(\Omega(n))^{2} \leqslant C \sum_{n>n_{0}}\left(\left|v_{-n}\right|^{2}+\left|v_{n}\right|^{2}\right)(\Omega(n))^{2}+C\|v\|_{\Omega}^{4} \sum_{n>n_{0}} \frac{64}{n^{2}}<\infty
$$

это доказывает соотношение (4.63).

Обратно, пусть $\left(\Delta_{n}\right)_{n \geqslant n_{0}} \in \ell^{2}(\Omega)$ с некоторым субмультипликативным весом $\Omega$, имеющим свойство (4.64) (или соответственно (4.66)). Тогда, по левому неравенству в (4.60) теоремы 66, мы имеем:

$$
\left(\Delta_{n}\right)_{n \geqslant n_{0}} \in \ell^{2}(\Omega) \Rightarrow\left(\left|\beta_{n}^{-}\left(z_{n}^{*}\right)\right|+\left|\beta_{n}^{+}\left(z_{n}^{*}\right)\right|\right)_{n \geqslant n_{0}} \in \ell^{2}(\Omega) .
$$

Ввиду определения отображения $A_{N}$ (см. (3.33) и (3.34)), отсюда вытекает, что $A_{N}(v) \in H(\Omega)$, поскольку при $n>N \quad \beta_{n}^{ \pm}\left(z_{n}^{*}\right)- \pm n$-е коэффициенты Фурье функции $A_{N}(v)$. Теперь, по предложению 53, мы получаем: $v \in H(\Omega)$ (или соответственно $\left.v \in H\left(e^{\varepsilon|n|}\right)\right)$. Это завершает доказательство теоремы 67 .

TEOPEMA 68. Если

$$
L=L^{0}+v(x), \quad L^{0}=i\left(\begin{array}{cc}
1 & 0 \\
0 & -1
\end{array}\right) \frac{d}{d x}, \quad v(x)=\left(\begin{array}{cc}
0 & P(x) \\
Q(x) & 0
\end{array}\right),
$$

- периодический оператор Дирака с $L^{2}$-потенциалом (т.е. $P$ u $Q$ - периодические $L^{2}([0, \pi])$-функции), то при $n \in \mathbb{Z},|n|>n_{0}(v)$, оператор $L$ имеет 6 круге с иентром $n$ и радиусом $r=1 / 4$ в точности два (с учетом их кратности) периодических (для четных $n$ ) или антипериодических (для нечетных $n$ ) собственных значения $\lambda_{n}^{+}$и $\lambda_{n}^{-}$и одно собственное значение Дирихле $\mu_{n}$.

Пусть

$$
\Delta_{n}=\left|\lambda_{n}^{+}-\lambda_{n}^{-}\right|+\left|\lambda_{n}^{+}-\mu_{n}\right|, \quad|n|>n_{0}
$$

тогда для любого субмультипликативного веса $\Omega$

$$
v \in H(\Omega) \Rightarrow\left(\Delta_{n}\right) \in \ell^{2}(\Omega) .
$$

Обратно, если $\Omega=(\Omega(n))_{n \in \mathbb{Z}}-$ такой субмультипликативный вес, что

$$
\frac{\log \Omega(n)}{n} \searrow 0, \quad \text { когда } n \rightarrow \infty
$$

mo

$$
\left(\Delta_{n}\right) \in \ell^{2}(\Omega) \Rightarrow v \in H(\Omega) .
$$

Если $\Omega$ - субмультипликативный вес экспоненциального типа, т.е.

$$
\lim _{n \rightarrow \infty} \frac{\log \Omega(n)}{n}>0
$$

mo

$$
\left(\Delta_{n}\right) \in \ell^{2}(\Omega) \Rightarrow \exists \varepsilon>0: \quad v \in H\left(e^{\varepsilon|n|}\right) .
$$


ДокАЗАТЕЛЬство. Существование такого $n_{0}=n_{0}(v)$, что $\lambda_{n}^{ \pm}$и $\mu_{n}$ вполне определены для $|n|>n_{0}$, непосредственно вытекает из теоремы 17 . Более того, без ограничения общности мы можем также считать $n_{0}$ настолько большим, что соотношение (4.60) в теореме 66 выполнено, если $|n|>n_{0}$.

Предположим, что $\Omega$ - субмультипликативный вес и $v \in H(\Omega)$. По правому неравенству в (4.60), мы имеем:

$$
\Delta_{n} \leqslant 54\left(\left|\beta_{n}^{-}\left(z_{n}^{*}\right)\right|+\left|\beta_{n}^{+}\left(z_{n}^{*}\right)\right|\right), \quad|n|>n_{0},
$$

и поэтому, по существу повторяя доказательство теоремы 42 , мы видим, что $\left(\Delta_{n}\right) \in \ell^{2}(\Omega)$.

Обратно, пусть $\left(\Delta_{n}\right)_{n \geqslant n_{0}} \in \ell^{2}(\Omega)$ при некотором субмультипликативном весе $\Omega$, имеющем свойство (4.70) (или соответственно (4.72)). Тогда по левому неравенству в (4.60) теоремы 66 мы имеем:

$$
\left(\Delta_{n}\right)_{n \geqslant n_{0}} \in \ell^{2}(\Omega) \Rightarrow\left(\left|\beta_{n}^{-}\left(z_{n}^{*}\right)\right|+\left|\beta_{n}^{+}\left(z_{n}^{*}\right)\right|\right)_{n \geqslant n_{0}} \in \ell^{2}(\Omega) .
$$

Ввиду определения отображения $A_{N}$ (см. (3.33)-(3.34)) это влечет: $A_{N}(v) \in$ $H(\Omega)$ при $N>n_{0}$, поскольку для $|n|>N \quad \beta_{n}^{+}\left(z_{n}^{*}\right)-n$-й коэффициент Фурье функции $\left(A_{N}(v)\right)^{21}$, а $\beta_{n}^{-}\left(z_{n}^{*}\right)-(-n)$-й коэффициент Фурье функции $\left(A_{N}(v)\right)^{12}$. Теперь, по предложению 57, мы имеем: $v \in H(\Omega)$ (или соответственно $v \in$ $\left.H\left(e^{\varepsilon|n|}\right)\right)$. Это завершает доказательство теоремы 68 .

4.5. Примечания. Базис в риссовском подпространстве $E_{n}$, построенный в [37; п. 3.3] в случае Хилла-Шрёдингера, служит далеким прототипом базиса в нашей лемме 59. Основные построения в этом разделе мотивированы техникой из [39], где разобран только случай Хилла-Шрёдингера, т.е. теорема 67, вместе с необходимыми предварительными неравенствами. Следует упомянуть, что для соболевских пространств, т.е. весов $\Omega(n)=|n|^{m}$, где $m$ - целое, это утверждение было доказано в [35], а для аналитических потенциалов - в [56].

Здесь же - и это одна из целей настоящей статьи - мы даем полное доказательство теоремы 68, ранее анонсированной в [18].

\section{5. Заключительные замечания}

5.1. Плотность конечнозонных потенциалов. Вещественнозначный потенциал называется конечнозонным потенииалом, если его последовательность спектральных лакун имеет только конечное число ненулевых членов (т.е. конечное число открытых лакун). В общем случае потенциал называется конечнозонным, если $\lambda_{n}^{+}=\lambda_{n}^{-}=\mu_{n}$ для всех, кроме конечного числа, $n \in \mathbb{N}$ в случае Хилла-Шрёдингера или $n \in \mathbb{Z}$ в случае Дирака. Оказывается, что для любого субмультипликативного веса $\Omega$ конечнозонные потенциалы всюду плотны в соответствующем соболевском пространстве потенциалов $H(\Omega)$ или $H_{D}(\Omega)$.

ТеОрема 69. Пусть $\Omega$ - субмультипликативный вес и $H(\Omega)$ - coответствующее соболевское пространство потенциалов Хилла-Шрёдингера. Тогда конечнозонные потенциалы всюду плотны в $H(\Omega)$. Более того, если функиия $v$ вещественнозначна, то она может быть аппроксимирована по $H(\Omega)$-норме сколь угодно близко вещественнозначными конечнозонными потенииалами. 
ДокАЗАтЕЛьство. Утверждение следует из лемм 40 и 51. Действительно, ввиду определения отображения $A_{N}$ и леммы 40, потенциал $v$-конечнозонный, если $A_{N}(v)$ - тригонометрический многочлен.

Зафиксируем $v \in H(\Omega)$ и $\varepsilon>0$. Выберем $N$ так, что $v \in \mathscr{B}_{r_{N} / 4}^{\Omega}$, и положим $w=A_{N}(v)$. Тогда, в силу (3.39),

$$
\|w\|_{\Omega}=\left\|A_{N}(v)\right\|_{\Omega}<3 r_{N} / 8 .
$$

Выберем тригонометрический многочлен $w^{*}$ так, что

$$
\left\|w-w^{*}\right\|_{\Omega}<\min \left(\varepsilon / 2, r_{N} / 8\right) .
$$

(Если $w=\sum_{k \in \mathbb{Z}} w_{k} e^{2 i k x}$, то можно выбрать $w^{*}=\sum_{|k| \leqslant M} w_{k} e^{2 i k x}$, где $M$ достаточно велико.) Конечно, если $w$ вещественнозначна, то $w^{*}$ можно тоже выбрать вещественнозначным. В силу (3.40), $A_{N^{-}}$образ шара $\mathscr{B}_{r_{N}}^{\Omega^{*}}$ накрывает шар $\mathscr{B}_{r_{N} / 2}^{\Omega^{*}}$. Более того, в силу замечания $52, A_{N}$-образ множества вещественнозначных потенциалов из $\mathscr{B}_{r_{N}}^{\Omega}$ накрывает множество вещественнозначных потенциалов из $\mathscr{B}_{r_{N} / 2}^{\Omega}$. Но так как

$$
\left\|w^{*}\right\|_{\Omega} \leqslant\left\|w^{*}-w\right\|_{\Omega}+\|w\|_{\Omega}<r_{N} / 2
$$

то конечнозонный потенциал $v^{*}=A_{N}^{-1}\left(w^{*}\right) \in \mathscr{B}_{r_{N}}^{\Omega}$ вполне определен, и вещественнозначен, если $w^{*}$ вещественнозначен, и, в силу (3.39),

$$
\left\|v^{*}-v\right\|_{\Omega} \leqslant \frac{3}{2}\left\|w^{*}-w\right\|_{\Omega}<\varepsilon
$$

Это завершает доказательство теоремы 69.

ТеОРема 70. Пусть $\Omega$ - субмультипликативный вес, $H_{D}(\Omega)$ - соответствующее соболевское пространство потенииалов Дирака. Тогда конечнозонные потенциаль всюду плотны в $H_{D}(\Omega)$. Более того, если

$$
v(x)=\left(\begin{array}{cc}
0 & P(x) \\
Q(x) & 0
\end{array}\right)
$$

- самосопряженный потенциал, т.е. $Q(x)=\overline{P(x)}$, то его можно аппроксимировать по $H_{D}(\Omega)$-норме сколь угодно близко самосопряженными конечнозонными потенииалами.

ДокАзАТЕльство. Ввиду определения отображения $A_{N}$ в случае Дирака из леммы 40 вытекает, что $v$ - конечнозонный потенциал, если элементы матрицы $A_{N}(v)$ - тригонометрические многочлены.

Зафиксируем потенциал Дирака

$$
v(x)=\left(\begin{array}{cc}
0 & P(x) \\
Q(x) & 0
\end{array}\right), \quad P(x)=\sum_{m \in \mathbb{Z}} P_{m} e^{2 i m x}, \quad Q(x)=\sum_{m \in \mathbb{Z}} Q_{m} e^{2 i m x},
$$

такой, что $v \in H(\Omega)$. Рассмотрим последовательность $x=\left(x_{m}\right)_{m \in \mathbb{Z}}$, где

$$
x_{m}=\left(\left|P_{m}\right|^{2}+\left|Q_{m}\right|^{2}\right)^{1 / 2} \Omega(m) .
$$


Так как $x \in \ell^{2}(\mathbb{Z})$, то, по лемме 48 , существует медленно растущий неограниченный вес $\Omega_{1}$ такой, что $x \in \ell^{2}\left(\mathbb{Z}, \Omega_{1}\right)$. Следовательно,

$$
\sum\left|x_{m}\right|^{2}\left(\Omega_{1}(m)\right)^{2}=\sum\left(\left|P_{m}\right|^{2}+\left|Q_{m}\right|^{2}\right)\left(\Omega_{1}(m) \Omega(m)\right)^{2}<\infty,
$$

т.е. $v \in H_{D}\left(\Omega^{*}\right)$, где $\Omega^{*}=\Omega_{1} \cdot \Omega$. Без потери общности мы можем предполагать, что

$$
\Omega(m) \leqslant C_{1}|m|
$$

(иначе, ввиду (3.7), мы можем заменить $\Omega_{1}$ на $\left(\Omega_{1}\right)^{1 / a}$, где $a$ - соответствующим образом выбранная положительная постоянная).

Теперь мы применим лемму 55 к весу $\Omega^{*}$. Зафиксируем $\varepsilon>0$ и выберем $N$ так, что $v \in \mathscr{B}_{r_{N} / 4}^{\Omega^{*}}$. Положим $w=A_{N}(v)$; тогда, в силу (3.61),

$$
\|w\|_{\Omega}=\left\|A_{N}(v)\right\|_{\Omega}<3 r_{N} / 8 .
$$

Выберем матрицу $\widetilde{w}(x)=\left(\begin{array}{cc}0 & \widetilde{w}^{12}(x) \\ \widetilde{w}^{21}(x) & 0\end{array}\right)$ так, что ее элементы $\widetilde{w}^{12}(x)$ и $\widetilde{w}^{21}(x)$ - тригонометрические многочлены и

$$
\|w-\widetilde{w}\|_{\Omega^{*}}<\min \left(\varepsilon / 2, r_{N} / 8\right) .
$$

Конечно, если $w$ - самосопряженный, то $\widetilde{w}$ можно тоже выбрать самосопряженным.

В силу (3.62), $A_{N}$-образ шара $\mathscr{B}_{r_{N}}^{\Omega^{*}}$ накрывает шар $\mathscr{B}_{r_{N} / 2}^{\Omega^{*}}$. Более того, в силу замечания $56, A_{N}$-образ множества самосопряженных потенциалов в $\mathscr{B}_{r_{N}}^{\Omega}$ накрывает множество самосопряженных потенциалов в $\mathscr{B}_{r_{N} / 2}^{\Omega}$.

Следовательно, так как

$$
\|\widetilde{w}\|_{\Omega^{*}} \leqslant\|\widetilde{w}-w\|_{\Omega^{*}}+\|w\|_{\Omega^{*}}<r_{N} / 2
$$

то конечнозонный потенциал $\widetilde{v}=A_{N}^{-1}(\widetilde{w}) \in \mathscr{B}_{r_{N}}^{\Omega^{*}}$ вполне определен, и он самосопряженный, если $\widetilde{w}$ - самосопряженный, и, в силу (3.61), мы имеем:

$$
\|\widetilde{v}-v\|_{\Omega} \leqslant\|\widetilde{v}-v\|_{\Omega^{*}} \leqslant \frac{3}{2}\|\widetilde{w}-w\|_{\Omega^{*}}<\varepsilon .
$$

Это завершает доказательство теоремы 70.

5.2. Примеры расходящихся разложений по собственным функциям. Хорошо известно (см. [57]-[59] в случае общих дифференциальных операторов или [48], [60] в случае Дирака), что спектральные разложения оператора $L=L^{0}+v, v \in L^{2}, b c=\operatorname{Per}^{+}$или $\mathrm{Per}^{-}$, рассматриваемые с блоками

$$
P_{*} f+\sum_{|k|>N} P_{k} f,
$$

сходятся в $L^{2}([0, \pi])$ (соответственно в $\left.\left(L^{2}([0, \pi])\right)^{2}\right)$ для любой $f \in L^{2}([0, \pi])$ (или $\left.f \in\left(L^{2}([0, \pi])\right)^{2}\right)$. В формуле $(5.1) P_{*}$ и $P_{k},|k|>N(k \in \mathbb{N}$ или $k \in \mathbb{Z})$, - риссовские проекторы, соответствующие локализации спектра, данной в теореме 9 для оператора Хилла-Шрёдингера и в теоремах 17 и 18 для оператора Дирака. 
Проекторы $P_{k},|k|>N$, двумерны, и если $\lambda_{k}^{+} \neq \lambda_{k}^{-}$, то $P_{k}$ можно записать в виде

$$
P_{k} f=\left\langle P_{k} f, \psi_{k}^{+}\right\rangle u_{k}^{+}+\left\langle P_{k} f, \psi_{k}^{-}\right\rangle u_{k}^{-},
$$

где

$$
P_{k} \psi_{k}^{ \pm}=\psi_{k}^{ \pm}, \quad L u_{k}^{ \pm}=\lambda_{k}^{ \pm} u_{k}^{ \pm}, \quad\left\|u_{k}^{ \pm}\right\|=1, \quad\left\langle u_{k}^{ \pm}, \psi_{k}^{ \pm}\right\rangle=1, \quad\left\langle u_{k}^{ \pm}, \psi_{k}^{\mp}\right\rangle=0 .
$$

Элементарное вычисление показывает, что

$$
\left\|\psi_{k}^{ \pm}\right\|=\frac{1}{\sqrt{1-\left|\left\langle u_{k}^{+}, u_{k}^{-}\right\rangle\right|^{2}}}
$$

Если мы постараемся раскрыть блоки в разложении (5.1), чтобы рассматривать разложение по собственным функциям, то, ввиду (5.4), такое разложение сходится тогда и только тогда, когда

$$
\sup _{k}\left\|\psi_{k}^{ \pm}\right\|=\sup _{k} \frac{1}{\sqrt{1-\left|\left\langle u_{k}^{+}, u_{k}^{-}\right\rangle\right|^{2}}}<\infty
$$

Теперь мы построим серию потенциалов $v$ так, что оператор $L=L^{0}+v$ имеет для достаточно больших $|k|$ простые (периодические и антипериодические) собственные значения $\lambda_{k}^{+} \neq \lambda_{k}^{-}$, но условие (5.5) не выполнено.

Построение.

В случае Хилла-Шрёдингера зафиксируем положительные числа $\left(\eta_{n}\right)$ так, что

$$
\eta_{n} \searrow 0, \quad n \eta_{n} \rightarrow \infty, \quad \text { когда } \quad n \rightarrow \infty,
$$

и выберем потенциал $w \in L^{2}([0, \pi])$ так, что

$$
w(x)=\sum_{k \in \mathbb{Z}} w_{k} e^{2 i k x}, \quad \frac{\left|w_{-n}\right|}{\left|w_{n}\right|}=\eta_{n}, \quad n \in \mathbb{N} .
$$

В случае Дирака зафиксируем последовательность положительных чисел $\left(\eta_{n}\right)_{n \in \mathbb{Z}}$ так, что

$$
\eta_{n} \searrow 0, \quad|n|^{1 / 2} \eta_{n} \rightarrow \infty, \quad \text { когда } \quad|n| \rightarrow \infty,
$$

и выберем потенциал $w(x)=\left(\begin{array}{cc}0 & w^{-}(x) \\ w^{+}(x) & 0\end{array}\right)$ так, что

$$
w^{ \pm}(x) \in L^{2}([0, \pi]), \quad w^{ \pm}(x)=\sum_{k \in \mathbb{Z}} w_{k}^{ \pm} e^{2 i k x}, \quad \frac{\left|w_{n}^{-}\right|}{\left|w_{n}^{+}\right|}=\eta_{n}, \quad n \in \mathbb{Z} .
$$

Так как условие (5.7) (соответственно (5.9)) не накладывает никаких требований на убывание коэффициентов Фурье функции $w(x)$ (соответственно функций $\left.w^{ \pm}(x)\right)$, мы можем выбрать $w$ в $(5.7)$ или $w^{ \pm}(x)$ в (5.9) как целые функции. Например, мы можем взять

$$
w_{n}=e^{-n^{2}}, w_{-n}=e^{-n^{2}} \eta_{n} \quad \text { и } \quad w_{n}^{+}=e^{-n^{2}}, w_{n}^{-}=e^{-n^{2}} \eta_{n} .
$$


Теорема 71. Для любого субмультипликативного веса $\Omega=(\Omega(m))_{m \in \mathbb{Z}}$ существует такой потенииал $v \in H(\Omega)$ (или $v \in H_{D}(\Omega)$ ), что соответствующий оператор Хилла-Шрёдингера (или Дирака) $L=L^{0}+v$ имеет при достаточно больших $|k|$ два простых собственных значения $\lambda_{k}^{+} \neq \lambda_{k}^{-}$около $\lambda_{k}^{0}$ и его разложение по собственным функииям расходится.

ДоказАТЕЛЬСтво. Так как неравенство $\widetilde{\Omega}(m) \geqslant \Omega(m)$ влечет за собой соотношение $H(\widetilde{\Omega}) \subset H(\Omega)$ или $H_{D}(\widetilde{\Omega}) \subset H_{D}(\Omega)$, мы можем без ограничения общности предполагать, что

$$
\Omega(m)=\Omega_{1}(m) \cdot e^{C|m|}, \quad \Omega_{1}(m)=m .
$$

Далее мы используем лемму 51 для случая Хилла-Шрёдингера или лемму 55 для случая Дирака и те же обозначения. Зафиксируем $N>N_{*}$ такое, что $w \in \mathscr{B}_{r_{N} / 2}^{\Omega}$. По лемме 51 (или лемме 55), существует единственный потенциал $v \in \mathscr{B}_{r_{N}}^{\Omega}$ такой, что

$$
A_{N}(v)=w .
$$

По определению отображения $A_{N}$ (см. (3.33) и (3.34) в случае Хилла-Шрёдингера и (3.52)-(3.54) в случае Дирака) и по (5.12) и (5.7) или (5.9), мы имеем:

$$
\frac{\left|\beta_{n}^{-}\left(z_{n}^{*}\right)\right|}{\left|\beta_{n}^{+}\left(z_{n}^{*}\right)\right|}=\eta_{n}, \quad \text { если } \quad|n|>N
$$

где

$$
z_{n}^{*}=\frac{1}{2}\left(z_{n}^{+}+z_{n}^{-}\right), \quad z_{n}^{ \pm}=\lambda_{n}^{ \pm}-\lambda_{n}^{0}
$$

или $z_{n}^{*}=z_{n}^{+}$, если $\lambda_{n}^{+}-$двойное собственное значение.

Оператор $L=L^{0}+v$ имеет при достаточно больших $|n|$ два различных собственных значения $\lambda_{n}^{+} \neq \lambda_{n}^{-}$около $\lambda_{n}^{0}$. Действительно, если $\lambda_{n}^{+}-$двойное собственное значение при некотором $n$, где $|n|>N$, то $z_{n}^{*}=z_{n}^{+}=\lambda_{n}^{+}-\lambda_{n}^{0}-$ двойной корень основного уравнения

$$
\left(\zeta_{n}(z)\right)^{2}-\beta_{n}^{+}(z) \beta_{n}^{-}(z)=0, \quad \zeta_{n}(z)=z-\alpha_{n}(z) .
$$

Следовательно, производная выражения слева обращается в нуль в точке $z_{n}^{*}$, т.е.

$$
2 \zeta_{n}\left(z_{n}^{*}\right)\left(1-\frac{d \alpha}{d z}\left(z_{n}^{*}\right)\right)=\frac{d \beta_{n}^{+}}{d z}\left(z_{n}^{*}\right) \cdot \beta_{n}^{-}\left(z_{n}^{*}\right)+\beta_{n}^{+}\left(z_{n}^{*}\right) \cdot \frac{d \beta_{n}^{-}}{d z}\left(z_{n}^{*}\right) .
$$

По оценкам производных $|d \alpha / d z|$ и $\left|d \beta^{ \pm} / d z\right|$ (см. лемму 29 в случае ХиллаШрёдингера и предложение 35 для случая Дирака), мы имеем:

$$
\sup _{\left[z_{n}^{-}, z_{n}^{+}\right]}\left|\frac{\partial \alpha_{n}}{\partial z}\right| \leqslant \varepsilon_{n}, \quad \sup _{\left[z_{n}^{-}, z_{n}^{+}\right]}\left|\frac{\partial \beta_{n}^{ \pm}}{\partial z}\right| \leqslant \varepsilon_{n}, \quad \varepsilon_{n} \downarrow 0,
$$

где

$$
\varepsilon_{n}= \begin{cases}O(1 / n) & \text { в случае Хилла-Шрёдингера, } \\ O(1 / \sqrt{|n|}) & \text { в случае Дирака. }\end{cases}
$$


В случае Хилла-Шрёдингера соотношение (5.16) немедленно вытекает из леммы 29. В случае Дирака, так как (5.11) влечет за собой $v \in H(\Omega) \subset$ $H\left(e^{C|m|}\right)$, то - в обозначениях предложения 35 - мы имеем:

$$
\left(\mathscr{E}_{|n|}(r)\right)^{2}=\sum_{|m|>|n|}|r(m)|^{2} \leqslant e^{-2 C|n|} \sum_{|m|>|n|}|r(m)|^{2} e^{2 C|m|} \leqslant e^{-2 C|n|}\|v\|_{H(\Omega)} .
$$

Тогда (2.110) в предложении 35 влечет за собой (5.16).

Теперь из (5.14) и (5.15) вытекает, что

$$
2\left|\zeta_{n}^{*}\left(z_{n}^{*}\right)\right|\left(1-\varepsilon_{n}\right) \leqslant \varepsilon_{n}\left(\left|\beta_{n}^{+}\left(z_{n}^{*}\right)\right|+\left|\beta_{n}^{-}\left(z_{n}^{*}\right)\right|\right) .
$$

Так как $\left|\zeta_{n}\left(z_{n}^{*}\right)\right|^{2}=\left|\beta_{n}^{+}\left(z_{n}^{*}\right)\right|\left|\beta_{n}^{-}\left(z_{n}^{*}\right)\right|$, то, в силу (5.13), последнее неравенство влечет за собой, что

$$
2\left(1-\varepsilon_{n}\right) \leqslant \frac{\varepsilon_{n}}{\sqrt{\eta_{n}}}\left(1+\eta_{n}\right)
$$

Но это неравенство не выполнено при больших $|n|$, поскольку - в силу (5.16) и (5.6) (или (5.8)) - его правая часть стремится к 0, в то время как его левая часть идет к 2 . Таким образом, $\lambda_{n}^{+} \neq \lambda_{n}^{-}$при достаточно больших $|n|$.

Далее мы покажем, что при достаточно больших $|n|$

$$
\frac{1}{4} \eta_{n} \leqslant \frac{\left|\beta_{n}^{-}\left(z_{n}^{ \pm}\right)\right|}{\left|\beta_{n}^{+}\left(z_{n}^{ \pm}\right)\right|} \leqslant 4 \eta_{n}
$$

Действительно, в силу (5.15), мы имеем:

$$
\left|\beta_{n}^{ \pm}\left(z_{n}^{ \pm}\right)-\beta_{n}^{ \pm}\left(z_{n}^{*}\right)\right| \leqslant \varepsilon_{n}\left|z_{n}^{+}-z_{n}^{-}\right| .
$$

С другой стороны, по лемме 40 и (5.13), мы имеем:

$$
\left|z_{n}^{+}-z_{n}^{-}\right| \leqslant 2\left(\left|\beta_{n}^{+}\left(z_{n}^{*}\right)\right|+\left|\beta_{n}^{-}\left(z_{n}^{*}\right)\right|\right)=2\left(1+\eta_{n}\right)\left|\beta_{n}^{+}\left(z_{n}^{*}\right)\right|=2\left(1+1 / \eta_{n}\right)\left|\beta_{n}^{-}\left(z_{n}^{*}\right)\right| .
$$

Тогда, в силу (5.16) и (5.6) (или (5.8)), $\varepsilon_{n}\left(1+1 / \eta_{n}\right) \rightarrow 0$ и $\varepsilon_{n}\left(1+\eta_{n}\right) \rightarrow 0$. Следовательно, при достаточно больших $|n|$ два последних неравенства влекут за собой:

$$
\left|\beta_{n}^{ \pm}\left(z_{n}^{ \pm}\right)-\beta_{n}^{ \pm}\left(z_{n}^{*}\right)\right| \leqslant \frac{1}{2}\left|\beta_{n}^{ \pm}\left(z_{n}^{*}\right)\right| .
$$

Отсюда вытекает, что

$$
\frac{1}{2}\left|\beta_{n}^{*}\left(z_{n}^{ \pm}\right)\right| \leqslant\left|\beta_{n}^{ \pm}\left(z_{n}^{ \pm}\right)\right| \leqslant 2\left|\beta_{n}^{ \pm}\left(z_{n}^{*}\right)\right|
$$

и это приводит нас к соотношению (5.17).

Пусть $u_{n}^{+}$и $u_{n}^{-}$- единичные собственные векторы оператора $L$, соответствующие собственным значениям $\lambda_{n}^{+}$и $\lambda_{n}^{-}$, т.е.

$$
L u_{n}^{ \pm}=\lambda_{n}^{ \pm} u_{n}^{ \pm}, \quad\left\|u_{n}^{ \pm}\right\|=1 .
$$

Положим

$$
\left(u_{n}^{+}\right)^{0}=P_{n}^{0} u_{n}^{+}, \quad\left(u_{n}^{-}\right)^{0}=P_{n}^{0} u_{n}^{-},
$$


где $P_{n}^{0}$ - ортогональный проектор на $n$-е собственное подпространство $E_{n}^{0}$ свободного оператора $L^{0}$. Тогда, по предложению 19, мы имеем:

$$
\left\|u_{n}^{ \pm}-\left(u_{n}^{ \pm}\right)^{0}\right\|=\left\|\left(P_{n}-P_{n}^{0}\right) u_{n}^{ \pm}\right\| \leqslant \kappa_{n} \rightarrow 0 .
$$

Так как $u_{n}^{ \pm}-\left(u_{n}^{ \pm}\right)^{0}$ ортогонален к $\left(u_{n}^{ \pm}\right)^{0}$, то мы получаем:

$$
\left\|\left(u_{n}^{ \pm}\right)^{0}\right\|^{2}=\left\|u_{n}^{ \pm}\right\|^{2}-\left\|u_{n}^{ \pm}-\left(u_{n}^{ \pm}\right)^{0}\right\|^{2} \geqslant 1-\kappa_{n}^{2}
$$

и

$$
\begin{aligned}
\left|\left\langle u_{n}^{+}, u_{n}^{-}\right\rangle\right| & \geqslant\left|\left\langle\left(u_{n}^{+}\right)^{0},\left(u_{n}^{-}\right)^{0}\right\rangle\right|-\left|\left\langle u_{n}^{+}-\left(u_{n}^{+}\right)^{0}, u_{n}^{-}-\left(u_{n}^{-}\right)^{0}\right\rangle\right| \\
& \geqslant\left|\left\langle\left(u_{n}^{+}\right)^{0},\left(u_{n}^{-}\right)^{0}\right\rangle\right|-\kappa_{n}^{2} .
\end{aligned}
$$

С другой стороны, по лемме $21,\left(u_{n}^{ \pm}\right)^{0}$ - собственные векторы операторов $P_{n}^{0} L^{0} P_{n}^{0}+S\left(\lambda_{n}^{ \pm}\right)$, соответствующие собственным значениям $\lambda_{n}^{ \pm}$; это приводит к соотношению

$$
\left(\begin{array}{cc}
\zeta\left(z_{n}^{ \pm}\right) & \beta_{n}^{-}\left(z_{n}^{ \pm}\right) \\
\beta_{n}^{+}\left(z_{n}^{ \pm}\right) & \zeta\left(z_{n}^{ \pm}\right)
\end{array}\right)\left(\begin{array}{c}
\left(u_{n}^{ \pm}\right)_{1}^{0} \\
\left(u_{n}^{ \pm}\right)_{2}^{0}
\end{array}\right)=0 .
$$

Следовательно,

$$
\zeta\left(z_{n}^{ \pm}\right) \cdot\left(u_{n}^{ \pm}\right)_{1}^{0}+\beta_{n}^{-}\left(z_{n}^{ \pm}\right) \cdot\left(u_{n}^{ \pm}\right)_{2}^{0}=0,
$$

откуда вытекает, что

$$
\left|\zeta\left(z_{n}^{ \pm}\right)\right| \cdot\left|\left(u_{n}^{ \pm}\right)_{1}^{0}\right|=\left|\beta_{n}^{-}\left(z_{n}^{ \pm}\right)\right| \cdot\left|\left(u_{n}^{ \pm}\right)_{2}^{0}\right| \cdot
$$

Так как $\left|\zeta\left(z_{n}^{ \pm}\right)\right|^{2}=\left|\beta_{n}^{-}\left(z_{n}^{ \pm}\right)\right| \cdot\left|\beta_{n}^{+}\left(z_{n}^{ \pm}\right)\right|$, то, в силу (5.13), мы получаем:

$$
\left|\left(u_{n}^{ \pm}\right)_{1}^{0}\right| \leqslant \sqrt{\left|\beta_{n}^{-}\left(z_{n}^{ \pm}\right)\right| /\left|\beta_{n}^{+}\left(z_{n}^{ \pm}\right)\right|} \cdot\left|\left(u_{n}^{ \pm}\right)_{2}^{0}\right| \leqslant 2 \sqrt{\eta_{n}} \cdot\left|\left(u_{n}^{ \pm}\right)_{2}^{0}\right| \leqslant 2 \sqrt{\eta_{n}} .
$$

Теперь, ввиду (5.18),

$$
1-\kappa_{n}^{2} \leqslant\left\|u_{n}^{ \pm}\right\|^{2}=\left|\left(u_{n}^{ \pm}\right)_{1}^{0}\right|^{2}+\left|\left(u_{n}^{ \pm}\right)_{2}^{0}\right|^{2} \leqslant\left|\left(u_{n}^{ \pm}\right)_{2}^{0}\right|^{2}+4 \eta,
$$

и, следовательно,

$$
\left|\left(u_{n}^{ \pm}\right)_{2}^{0}\right| \geqslant \sqrt{1-\kappa_{n}^{2}-4 \eta} .
$$

Отсюда вытекает, что

$$
\begin{aligned}
\left|\left\langle\left(u_{n}^{+}\right)^{0},\left(u_{n}^{-}\right)^{0}\right\rangle\right| & =\left|\left(u_{n}^{+}\right)_{2}^{0} \cdot\left(u_{n}^{-}\right)_{2}^{0}+\left(u_{n}^{+}\right)_{1}^{0} \cdot\left(u_{n}^{-}\right)_{1}^{0}\right| \\
& \geqslant\left|\left(u_{n}^{+}\right)_{2}^{0}\right| \cdot\left|\left(u_{n}^{-}\right)_{2}^{0}\right|-\left|\left(u_{n}^{+}\right)_{1}^{0}\right| \cdot\left|\left(u_{n}^{-}\right)_{1}^{0}\right| \geqslant 1-\kappa^{2}-8 \eta .
\end{aligned}
$$

Таким образом, ввиду (5.19),

$$
1 \geqslant\left|\left\langle u_{n}^{+}, u_{n}^{-}\right\rangle\right| \geqslant 1-2 \kappa^{2}-8 \eta \rightarrow 1, \quad \text { когда } \quad|n| \rightarrow \infty,
$$

откуда вытекает, что

$$
\left|\left\langle u_{n}^{+}, u_{n}^{-}\right\rangle\right| \rightarrow 1 \text {. }
$$

Следовательно, ввиду (5.5), разложение по собственным функциям оператора $L=L^{0}+v$ не сходится. Теорема 71 доказана. 
5.3. Двучленные потенциалы. В случае специальных потенциалов, например, потенциала Матье

$$
v(x)=2 a \cos 2 x, \quad a \neq 0, \quad a \in \mathbb{R},
$$

или общих тригонометрических многочленов

$$
v(x)=\sum_{-N}^{N} c_{k} \exp (i k x), \quad c_{k}=\overline{c_{-k}}, \quad 0 \leqslant k \leqslant N<\infty,
$$

мы приходим к двум группам вопросов:

(i) Закрыта ли $n$-я зона неустойчивости (спектральная лакуна), т.е.

$$
\gamma_{n}=\lambda_{n}^{+}-\lambda_{n}^{-}=0
$$

или, в эквивалентной форме, равна ли двум кратность собственного значения $\lambda_{n}^{+}$?

(ii) Если $\gamma_{n} \neq 0$, можем ли мы сказать больше о размере этих лакун, или, при достаточно больших $|n|$, каково асимптотическое поведение последовательности $\gamma_{n}=\gamma_{n}(v)$ ?

Э.Л. Инс [61] дал отрицательный ответ на вопрос (i) в случае потенциала (5.20): оператор Матье-Хилла имеет только простые собственные значения как для периодических, так и для антипериодических граничных условий, т.е. все зоны неустойчивости оператора Матье-Шрёдингера открыты. Его доказательство приведено в [20]; см. другие доказательства этого факта в [62]-[64] и дальнейшую библиографию в [20], [65], [22].

При фиксированном $n$, если $a \rightarrow 0$, в случае потенциала (5.20) Д. Леви и Дж. Келлер [66] дали асимптотику $\gamma_{n}=\gamma_{n}(a)$, именно,

$$
\gamma_{n}=\lambda_{n}^{+}-\lambda_{n}^{-}=\frac{8(|a| / 4)^{n}}{[(n-1) !]^{2}}(1+O(a)) .
$$

Почти двадцатью годами позже Э. Харрелл [25] нашел, с точностью до постоянного множителя, асимптотику спектральных лакун в случае потенциала Матье (5.20), когда $n \rightarrow \infty$. Й. Аврон и Б. Саймон [26] дали иное доказательство асимптотик Харрелла и нашли точное значение постоянного множителя, что приводит к следующей формуле:

$$
\gamma_{n}=\lambda_{n}^{+}-\lambda_{n}^{-}=\frac{8(|a| / 4)^{n}}{[(n-1) !]^{2}}\left(1+O\left(\frac{1}{n^{2}}\right)\right) .
$$

Упомянем, что в [67], [68] мы нашли как асимптотики спектральных лакун одномерного оператора Дирака с косинус-потенциалом, так и кратности всех периодических и антипериодических собственных значений.

Теорема 72. Пусть $L$ - оператор Дирака (1.9), рассматриваемый с потенииалом

$$
v(x)=\left(\begin{array}{cc}
0 & 2 b \cos 2 x \\
2 b \cos 2 x & 0
\end{array}\right), \quad 0 \leqslant x \leqslant \pi,
$$


u пусть $\gamma_{n}, n \in \mathbb{Z},-$ его последовательность спектральных лакун. Тогда $\gamma_{-n}=\gamma_{n}$ для всех $n \in \mathbb{Z} u \gamma_{n}=0$ для четных $n$, но $\gamma_{n}>0$ для нечетных $n$. Более того, для нечетных $n=2 m+1 \rightarrow+\infty$ имеет место асимптотика

$$
\gamma_{n}=2 b\left(\frac{b}{4}\right)^{n-1}\left[\left(\frac{n-1}{2}\right) !\right]^{-2}\left[1+O\left(\frac{\log n}{n}\right)\right] .
$$

Эта теорема есть комбинация теоремы 1 в [67] и теоремы 14 в [68]. Подчеркнем, что сейчас мы рассматриваем оператор $L$ на отрезке $[0, \pi]$, в то время как в [67], [68] все операторы рассматриваются на отрезке $[0,1]$, так что в $(5.25)$ коэффициенты перенормированы соответствующим образом.

Й. Аврон и Б. Саймон [26] подняли вопрос об асимптотике спектральных лакун в случае двучленного потенциала

$$
v(x)=a \cos 2 x+b \cos 4 x .
$$

А. Грижис [69] задал по существу тот же вопрос для изоспектрального потенциала $v(x)=a \sin 2 x-b \cos 4 x$.

Мы нашли эти асимптотики. Наши результаты (см. ниже) анонсированы в [28]; их подробные доказательства можно найти в [29].

Для вещественных $a, b \neq 0$ выберем $\alpha$ и $t$ так, что

$$
a=-4 \alpha t, \quad b=-2 \alpha^{2},
$$

где либо

$$
\text { оба числа } \alpha \text { и } t \text { вещественны (если } b<0) \text {, }
$$

либо

$$
\text { оба числа } \alpha \text { и } t \text { чисто мнимые (если } b>0 \text { ). }
$$

Эта параметризация приходит от Э. Уиттакера [70] и Э. Л. Инса [24]. Подробное обсуждение этого потенциала можно найти в [22; п. 7.9] и в наших статьях [71], [29]. Его замечательные свойства предопределены тем фактом, как это поняли в 50-е годы В. Магнус и С. Уинклер [65], что при целых $t$ возникают квазиточно решаемые уравнения (см. [72], [73] об общей теории квазиточно решаемых уравнений).

При анализе потенциалов (5.26) специальная роль целых $t$ проявляется в структуре коэффициентов в асимптотике $\gamma_{n}(\alpha), \alpha \rightarrow 0$, и $\gamma_{n}(v), n \rightarrow \infty$. Именно (см. теорему 7 в [29]), если $t$ и $n$ зафиксированы, то для четных $n$

$$
\gamma_{n}=\left|\frac{8 \alpha^{n}}{2^{n}[(n-1) !]^{2}} \prod_{k=1}^{n / 2}\left(t^{2}-(2 k-1)^{2}\right)\right|(1+O(\alpha)),
$$

а для нечетных $n$

$$
\gamma_{n}=\left|\frac{8 \alpha^{n} t}{2^{n}[(n-1) !]^{2}} \prod_{k=1}^{(n-1) / 2}\left(t^{2}-(2 k)^{2}\right)\right|(1+O(\alpha)) .
$$


Эти формулы можно сравнить с данным выше утверждением (5.23). (При фиксированном $n$ асимптотики спектральных лакун $\gamma_{n}(\varepsilon), \varepsilon \rightarrow 0$, для более общих уравнений Инса

$$
(1+\varepsilon a \cos (2 x)) y^{\prime \prime}+\varepsilon b \sin (2 x) y+\varepsilon c(\cos 2 x) y=0
$$

и уравнений Ламе [22; гл. 7] даны Г. Волкмером [74].)

Если же зафиксированы $\alpha \neq 0, t \neq 0$, то (см. теорему 9 в [29]) при $n \rightarrow \infty$ выполнена следующая асимптотическая формула:

для четных $n$

$$
\gamma_{n}=\frac{8|\alpha / 2|^{n}}{[2 \cdot 4 \cdots(n-2)]^{2}}\left|\cos \left(\frac{\pi}{2} t\right)\right|\left[1+O\left(\frac{\log n}{n}\right)\right],
$$

для нечетных $n$

$$
\gamma_{n}=\frac{8|\alpha / 2|^{n}}{[1 \cdot 3 \cdots(n-2)]^{2}} \frac{2}{\pi}\left|\sin \left(\frac{\pi}{2} t\right)\right|\left[1+O\left(\frac{\log n}{n}\right)\right] .
$$

Этот результат можно сравнить с приведенной выше формулой (5.24).

Наши асимптотики (5.28)-(5.29) приводят к интересным тождествам для квадратов целых чисел (см. [29; раздел 4, теорема 8]).

Наши доказательства основаны на общей асимптотической формуле для "лакун" $\left|\gamma_{n}\right|$ в терминах коэффициентов Фурье потенциала

$$
v(x)=\sum_{k \text { четные }} V_{k} e^{i k x}, \quad x \in[0, \pi] .
$$

ТЕОРема 73. Пусть $v$-вещественнозначный потенииал.

(а) Если $\|v\| \leqslant 1 / 9$, то для каждого $n=1,2, \ldots$ существует точка $z=z_{n}$ такая, что

$$
|z| \leqslant 4\|v\|
$$

u

$$
2\left|\beta_{n}^{+}(z)\right|\left(1-3\|v\|^{2} / n^{2}\right) \leqslant \gamma_{n} \leqslant 2\left|\beta_{n}^{+}(z)\right|\left(1+3\|v\|^{2} / n^{2}\right) .
$$

(b) Eсли $V(0)=\frac{1}{\pi} \int_{0}^{\pi} v(x) d x=0$, то существует такое $N_{0}=N_{0}(v)$, что (5.33) выполнено для $n \geqslant N_{0}$, где выбрано $z=z_{n}$,

$$
\left|z_{n}\right|<1
$$

Это утверждение является более точной формой теоремы 50 в случае оператора Хилла-Шрёдингера с вещественнозначным потенциалом. Его доказательство можно найти в [29; теорема 6].

Формула (5.33) дает двусторонние оценки для $\left|\gamma_{n}\right|$. Но даже оценки для $\left|\gamma_{n}\right|$ сверху в формуле (2.129) из леммы 40 могут дать ценную информацию. Рассмотрим оператор Хилла-Шрёдингера с потенциалом $v$ в классе Харди как М. Г. Гасымов [30], т.е.

$$
v(x)=\sum_{k=1}^{\infty} V(2 k) e^{2 i k x}
$$


или, по аналогии, для операторов Дирака

$$
v(x)=\left(\begin{array}{cc}
0 & P(x) \\
Q(x) & 0
\end{array}\right), \quad P(x)=\sum_{k=1}^{\infty} p(2 k) e^{2 i k x}, \quad Q(x)=\sum_{k=1}^{\infty} q(2 k) e^{2 i k x} .
$$

ТеОрема 74. Если L - оператор Хилла-Шрёдингера с потенииалом $v$ вида (5.35) или оператор Дирака с потенциалом $v$ вида (5.36), то тогда все периодические и антипериодические собственные значения оператора $L$ имеют алгебрачческую кратность не менъше 2 .

ДокАзАтельство (наьросок). В случае Хилла-Шрёдингера утверждение вытекает из результатов Гасымова [30] (см. дальнейшее развитие в [75]-[77]). Но иное доказательство можно дать, используя лемму 40. Это доказательство работает и в случае Дирака, так что мы рассмотрим только этот - по-видимому, не изучавшийся до сих пор, - случай.

Формулы (2.67)-(2.77) для матричных элементов $S^{i j}$ могут быть использованы, если $|n| \geqslant N_{*}$, где $N_{*}$ выбрано так, что $\left\|\widehat{T}_{n}^{2}\right\| \leqslant 1 / 2$ (см. лемму 33 и п. 2.4). Ввиду (2.90), мы имеем эти формулы

(i) для индивидуального потенциала $v$, если $|n| \geqslant N_{*}(v)$, где $N_{*}(v)$ выбрано так, чтобы правая часть в (2.103) не превосходила $1 / 2$, или

(ii) для всех $n \in \mathbb{Z}$ и всех $v$ при $\|v\| \leqslant \rho$, если $\rho=\frac{1}{2} C^{-1 / 2}$, где $C$ - абсолютная постоянная, приходящая из (2.103).

В обоих случаях мы можем использовать формулы (2.67)-(2.77). Предположение (5.36) означает, что

$$
p(k)=0, \quad q(k)=0 \quad \text { для } \quad k \leqslant 0 .
$$

Если $n \geqslant 0$ (и $n \geqslant N_{*}$ в (i)), то мы используем первое из соотношений (2.75), а именно $S_{0}^{12}=p(-2 n)=0$, а затем $(2.76)$, чтобы прийти к выводу, что все $S_{2 \nu}^{12}=0$ и, тем самым,

$$
S^{12}(v ; n, z)=0 \text {, если } n \geqslant 0 \text { в случае (ii) (или } n \geqslant N_{*} \text { в случае (i)). }
$$

Действительно, если $\nu \geqslant 1$, то каждый член в сумме (2.76), дающей нам определение элемента $S_{2 \nu}^{12}$, обращается в нуль как кратное произведения

$$
p\left(-n-j_{1}\right) q\left(j_{1}+j_{2}\right) p\left(-j_{2}-j_{3}\right) q\left(j_{3}+j_{4}\right) \cdots q\left(j_{2 \nu-1}+j_{2 \nu}\right) p\left(-j_{2 \nu}-n\right) .
$$

Это произведение обращается в нуль, поскольку

$$
\left(-n-j_{1}\right)+\left(j_{1}+j_{2}\right)+\left(-j_{2}-j_{3}\right)+\cdots+\left(j_{2 \nu-1}+j_{2 \nu}\right)+\left(-j_{2 \nu}-n\right)=-2 n \leqslant 0
$$

и по крайней мере один из целочисленных индексов неположителен, но тогда соответствующий множитель в произведении равен нулю.

Если же $n<0$, то мы используем вторую часть в $(2.75)$ и (2.77) чтобы теми же самыми рассуждениями объяснить, что

$$
\left.S^{21}(v ; n, z)=0 \text {, если } n \leqslant 0 \text { (или }-n \geqslant N_{*}\right) .
$$


По лемме 30, мы имеем: $S^{11}(v ; n, z)=S^{22}(v ; n, z)$, так что для всех $n$, удовлетворяющих условию (i) (или (ii)), основное уравнение (2.57) приобретает вид

$$
\left(z-S^{11}(v ; n, z)\right)^{2}=0,
$$

и его два корня совпадают: $z_{n}^{+}=z_{n}^{-}$. Следовательно, $\lambda_{n}^{+}=\lambda_{n}^{-}$.

Используя аналитическое продолжение функций

$$
\left(\lambda_{j}^{+}(z)-\lambda_{j}^{-}(z)\right)^{2} \quad \text { и } \quad \lambda_{j}^{+}(z)+\lambda_{j}^{-}(z),
$$

возникающих из семейства потенциалов

$$
v_{z}(x)=z \cdot v(x), \quad z \in \mathbb{C},
$$

мы можем объяснить для потенциалов Дирака вида (5.36), что все периодические и антипериодические собственные значения имеют алгебраическую кратность не меньше 2. Теорема 74 доказана.

Заметим, что опущенные детали в этом доказательстве аналогичны (но не тождественны) построениям раздела 5 в нашей статье [78], где собственные значения были простые, а не двойные.

5.4. Заключение. В заключение напомним, что в этой статье мы рассматриваем классы потенциалов $v \in H(\Omega)$ для случая Хилла-Шрёдингера или $v \in H_{D}(\Omega)$ в случае Дирака, лежащие между $L^{2}$ и $A$ (пространством аналитических функций на оси $\mathbb{R})$. В терминах весов $\Omega$ вида (3.1), (3.2) это означает, что

$$
\Omega(n) \geqslant 1 \quad \text { и } \quad \frac{\log \Omega(n)}{n} \leqslant C<\infty, \quad n \in \mathbb{N} .
$$

Если же мы отказываемся от этих ограничений, то, с одной стороны, мы должны рассмотреть сингулярные потенциалы или соответственно такие веса $\Omega$, что $\Omega(n) \rightarrow 0$. Это можно сделать для случая Хилла-Шрёдингера, но надо требовать, по крайней мере, чтобы $v$ принадлежал отрицательному соболевскому пространству $H^{-1}$, т.е., в терминах весов, чтобы

$$
n \cdot \Omega(n) \geqslant c>0 .
$$

С другой стороны, если $\log \Omega(n) / n \rightarrow \infty$, то каждый потенциал $v(x) \in H(\Omega)$ есть ограничение на $\mathbb{R}$ целой функции $v(z), z \in \mathbb{C}$. Целые (периодические) функции конечного порядка, скажем, $\rho>0$, определяются как функции, удовлетворяющие неравенству

$$
|v(z)| \leqslant A \exp \left(a|y|^{\rho}\right), \quad z=x+i y
$$

или, в терминах весов, как функции из пространства $H(\Omega)$, где

$$
\Omega(n)=\exp \left(b|n|^{\beta}\right), \quad \beta=1+1 / \rho .
$$

Напомним наши результаты из [79], а именно теоремы 5 и 7 там, в случае Хилла-Шрёдингера. 
ПРЕДЛОЖЕНИЕ 75. Пусть $\Omega$ дано в (5.42); тогда

$$
v \in H(\Omega) \Rightarrow \exists c>0: \sup \left(\left|\gamma_{n}\right|+\left|\delta_{n}\right|\right) \Omega_{c}(n)<\infty,
$$

¿əe

$$
\Omega_{c}(n)=\exp \left(c n(\log n)^{1 /(1+\rho)}\right) .
$$

Это утверждение неулучшаемо, с точностью до типов $a, b$ и $c$ в (5.41), (5.42) и (5.44) соответственно, в следующем смысле.

ПРЕДЛОЖЕНИЕ 76. Существуют вещественнозначные потенциаль $v(x)$ такие, что $v(x)$ - целая функция порядка $\rho$, но спектральные лакуны $\gamma_{n}=\lambda_{n}^{+}-\lambda_{n}^{+}$удовлетворяют неравенству

$$
\gamma_{n} \geqslant \exp \left(-d n(\log n)^{1 /(1+\rho)}\right), \quad \text { əde } \quad d>0,
$$

для бесконечного числа индексов $n$.

Потенциалы $v(x)$, которые являются целыми функциями, имеют еще одно свойство, контрастирующее с теоремой 69.

ПрЕДЛОжЕНИЕ 77. Если $v(z)-$ целая функиия, $v(z+\pi)=v(z)$, mо $v(x)$ не есть конечнозонный потенциал.

Доказательство этого утверждения было сообщено нам Петром Гриневичем в апреле 2002 г. Оно основано на анализе уравнения Дубровина в комплексной плоскости: его решение с необходимостью перестает существовать в ограниченной части $\mathbb{C}$. Мы не вдаемся сейчас в детали.

Основные результаты этого обзора в случае Хилла-Шрёдингера могут быть распространены на сингулярные потенциалы $v \in H(\Omega)$ с условием (5.40). Мы дадим эти конструкции и результаты в статье, которая готовится к публикации.

5.5. Примечания. Вопрос о плотности в $L^{2}$ конечнозонных (вещественных) потенциалов оператора Шрёдингера был поставлен С. П. Новиковым [7]; доказательство было дано В. А. Марченко и И. В. Островским [80]. Т. Мисюра [81] использовала методы из [80] чтобы доказать $L^{2}$-плотность конечнозонных потенциалов в случае операторов Дирака с симметричными матричными потенциалами. В. Ткаченко [82], [83] доказал то же в случае кососимметрических потенциалов.

Б. Митягин (в неопубликованной рукописи, лето 2000 г.) рассмотрел комплекснозначные потенциалы в случае Хилла-Шрёдингера и произвольных пространств $H(\Omega)$ с субмультипликативными весами, используя балансирование "голова-хвост" и соответствующие сжимающие отображения. Его подход был использован в [84] для доказательства плотности конечнозонных потенциалов оператора Дирака в $H(\Omega)$ при специальных ограничениях на веса $\Omega$. Tеоремы 69, 70 покрывают все случаи, когда вес $\Omega$ - субмультипликативный. Последнее ограничение - по существу дела, поскольку (см. предложение 77) в классах целых функций $H(\Omega)$, где $\Omega$ дано в (5.44), нет конечнозонных потенииалов.

Наши результаты п. 5.2, в частности теорема 71 , были анонсированы в заметке [46] одного из авторов.

Дальнейшие литературные примечания даны в тексте этого раздела. 


\section{6. Дополнение}

Лемма 78. Для каждого $n \in \mathbb{N}$ выполнено

$$
\begin{aligned}
& \sum_{k \neq \pm n} \frac{1}{\left|n^{2}-k^{2}\right|}<\frac{2 \log 6 n}{n} \\
& \sum_{k \neq \pm n} \frac{1}{\left|n^{2}-k^{2}\right|^{2}}<\frac{4}{n^{2}} .
\end{aligned}
$$

Доказательство опускается, посколько оно элементарно.

Нам нужно также следующее утверждение.

Лемма 79. Существует абсолютная постоянная $C>0$ такая, что (a) если $n \in \mathbb{N} u b \geqslant 1$, то

$$
\sum_{k} \frac{1}{\left|n^{2}-k^{2}\right|+b} \leqslant C \frac{\log 6 b}{\sqrt{b}}
$$

(b) если $n \geqslant 0$ u $b>0$, mo

$$
\sum_{k \neq \pm n} \frac{1}{\left|n^{2}-k^{2}\right|^{2}+b^{2}} \leqslant \frac{C}{\left(n^{2}+b^{2}\right)^{1 / 2}\left(n^{4}+b^{2}\right)^{1 / 4}} .
$$

ДокАЗАТЕЛЬСтво. (а) Если $b<2 n^{2}$, то $n>\sqrt{b / 2}$, и поэтому, ввиду (6.1), мы имеем

$$
\sum_{k} \frac{1}{\left|n^{2}-k^{2}\right|+b} \leqslant \frac{2}{b}+\sum_{k \neq \pm n} \frac{1}{\left|n^{2}-k^{2}\right|} \leqslant \frac{2}{b}+2 \frac{\log 6 n}{n} \leqslant \frac{2}{b}+\frac{4 \log 6 b}{\sqrt{b}} .
$$

Если $b \geqslant 2 n^{2}$, то

$$
\begin{aligned}
\sum_{k} \frac{1}{\left|n^{2}-k^{2}\right|+b} & \leqslant 2 \sum_{k=0}^{n} \frac{1}{n^{2}-k^{2}+b}+2 \sum_{k=n+1}^{\infty} \frac{1}{k^{2}-n^{2}+b} \\
& \leqslant 2(n+1) \frac{1}{b}+2 \sum_{k=n+1}^{\infty} \frac{1}{k^{2}+b / 2} \leqslant 2 \frac{\sqrt{b}+1}{b}+2 \int_{n}^{\infty} \frac{1}{x^{2}+b / 2} d x \\
& \leqslant \frac{4}{\sqrt{b}}+4 \frac{\pi}{\sqrt{b}}
\end{aligned}
$$

что завершает доказательство неравенства (6.3).

(b) Имеем:

$$
\sum_{k \neq \pm n} \frac{1}{\left|n^{2}-k^{2}\right|^{2}+b^{2}} \leqslant \int_{-\infty}^{\infty} \frac{1}{\left(x^{2}-n^{2}\right)^{2}+b^{2}} d x
$$

Стандартное вычисление вычетов показывает, что

$$
\int_{-\infty}^{\infty} \frac{1}{\left(x^{2}-n^{2}\right)^{2}+b^{2}} d x \leqslant \frac{\pi}{b \rho}
$$


где

$$
\rho=\left(n^{4}+b^{2}\right)^{1 / 4} .
$$

Действительно, функция $f(z)=\left(\left(z^{2}-n^{2}\right)^{2}+b^{2}\right)^{-1}$ имеет два полюса $\zeta_{1}$ и $\zeta_{2}$ в верхней полуплоскости. Так как

$$
R \sup _{|z|=R}|f(z)| \rightarrow 0, \quad \text { когда } \quad R \rightarrow \infty,
$$

то хорошо известное рассуждение из комплексного анализа показывает, что

$$
\int_{-\infty}^{\infty} f(x) d x=2 \pi i\left(\operatorname{Res}_{\zeta_{1}} f+\operatorname{Res}_{\zeta_{2}} f\right)
$$

Пусть $n^{2} \pm i b=\rho^{2} e^{ \pm i \varphi}, 0 \leqslant \varphi \leqslant \pi / 2$; тогда выполнено

$$
\left(z^{2}-n^{2}\right)^{2}+b^{2}=\left(z^{2}-n^{2}-i b\right)\left(z^{2}-n^{2}+i b\right)=\left(z-\zeta_{1}\right)\left(z+\zeta_{1}\right)\left(z-\bar{\zeta}_{1}\right)\left(z+\bar{\zeta}_{1}\right),
$$

где $\zeta_{1}=\rho \exp (i \varphi / 2)$. Поэтому $\zeta_{2}=-\bar{\zeta}_{1}$ и

$$
\operatorname{Res}_{\zeta_{1}} f+\operatorname{Res}_{\zeta_{2}} f=\frac{e^{-i \varphi / 2}}{4 i \rho b}+\frac{e^{i \varphi / 2}}{4 i \rho b}=\frac{\cos (\varphi / 2)}{2 i \rho b} .
$$

Следовательно,

$$
\int_{-\infty}^{\infty} f(x) d x=\frac{\pi}{\rho b} \cos (\varphi / 2) \leqslant \frac{\pi}{\rho b}=\frac{\pi}{b\left(n^{4}+b^{2}\right)^{1 / 4}},
$$

и мы получаем

$$
\sum_{k \neq \pm n} \frac{1}{\left|n^{2}-k^{2}\right|^{2}+b^{2}} \leqslant \frac{\pi}{b\left(n^{4}+b^{2}\right)^{1 / 4}}
$$

Так как

$$
\frac{1}{\left(n^{2}-k^{2}\right)^{2}+b^{2}} \leqslant \frac{2}{\left(n^{2}-k^{2}\right)^{2}+n^{2}+b^{2}}, \quad k \neq \pm n,
$$

то из (6.5)вытекает

$$
\sum_{k \neq \pm n} \frac{1}{\left|n^{2}-k^{2}\right|^{2}+b^{2}} \leqslant 2 \sum_{k \neq \pm n} \frac{1}{\left|n^{2}-k^{2}\right|^{2}+\widetilde{b}^{2}} \leqslant \frac{2 \pi}{\widetilde{b}\left(n^{4}+\widetilde{b}^{2}\right)^{1 / 4}},
$$

где $\widetilde{b}=\sqrt{n^{2}+b^{2}}$. Это доказывает, что (6.4) выполнено с постоянной $C=2 \pi$. Лемма 79 доказана.

\section{Список литературы}

[1] И. М. Гельфанд, Б. М. Левитан, “Об определении дифференциального уравнения по его спектральной функции”, Докл. АН СССР, 77:4 (1951), 557-560.

[2] M. J. Ablowitz, D. J. Kaup, A. C. Newell, H. Segur, "The inverse scattering transform - Fourier analysis for nonlinear problems", Stud. Appl. Math., 53:4 (1974), 249-315.

[3] Б. А. Дубровин, В.А. Матвеев, С.П. Новиков, "Нелинейные уравнения типа Кортевега-де Фриза, конечнозонные линейные операторы и абелевы многообразия", УМН, 31:1 (1976), 55-136. 
[4] Б. А. Дубровин, И. М. Кричевер, С. П. Новиков, "Интегрируемые системы. I", Динамические системы - 4, Итоги науки и техники. Соврем. проблемы матем. Фунд. напр., 4, ВИНИТИ, М., 1985, 179-285.

[5] C. S. Gardner, J. M. Greene, M. D. Kruskal, R. M. Miura, "Method for solving the Korteweg-de Vries equation", Phys. Rev. Lett., 19 (1967), 1095-1097.

[6] C.S. Gardner, J. M. Greene, M. D. Kruskal, R. M. Miura, "Korteweg-de Vries equation and generalization. VI. Method for exact solution", Comm. Pure Appl. Math., 27 (1974), 97-133.

[7] С. П. Новиков, "Периодическая задача для уравнения Кортевега-де Фриза. I", Функи. анализ и его прилож., 8:3 (1974), 54-66.

[8] В.Е. Захаров, А.Б. Шабат, "Схема интегрирования нелинейных уравнений математической физики методом обратной задачи рассеяния. I", Функи. анализ и его прилож., 8:3 (1974), 43-53.

[9] H. Hochstadt, "Estimates of the stability intervals for Hill's equation", Proc. Amer. Math. Soc., 14 (1963), 930-932.

[10] H. Hochstadt, "On the determination of a Hill's equation from its spectrum", Arch. Rational Mech. Anal., 19 (1965), 353-362.

[11] В. Ф. Лазуткин, Т. Ф. Панкратова, "Асимптотика ширины лакун в спектре оператора Штурма-Лиувилля с периодическим потенциалом", Докл. АН CCCP, 215:5 (1974), 1048-1051.

[12] В.А. Марченко, И. В. Островский, "Характеристика спектра оператора Хилла", Матем. сб., 97 (139): 4 (1975), 540-606.

[13] H. P. McKean, E. Trubowitz, "Hill's operator and hyperelliptic function theory in the presence of infinitely many branch points", Comm. Pure Appl. Math., 29:2 (1976), $143-226$.

[14] Б. А. Дубровин, "Обратная задача теории рассеяния для периодических конечнозонных потенциалов", Функи. анализ и его прилож., 9:1 (1975), 65-66.

[15] E. Trubowitz, "The inverse problem for periodic potentials", Comm. Pure Appl. Math., 30:3 (1977), 321-337.

[16] B. Grébert, T. Kappeler, B. Mityagin, "Gap estimates of the spectrum of the Zakharov-Shabat system", Appl. Math. Lett., 11:4 (1998), 95-97.

[17] B. Grébert, T. Kappeler, "Estimates on periodic and Dirichlet eigenvalues for the Zakharov-Shabat system", Asymptotic Anal., 25:3-4 (2001), 201-237; "Erratum", Asymptotic Anal., 29:2 (2002), 183.

[18] P. Djakov, B. Mityagin, "Spectra of 1-D periodic Dirac operators and smoothness of potentials", C. R. Math. Acad. Sci. Soc. R. Can., 25:4 (2003), 121-125.

[19] P. Djakov, B. Mityagin, "Instability zones of a periodic 1D Dirac operator and smoothness of its potential", Comm. Math. Phys., 259:1 (2005), 139-183.

[20] M. S. P. Eastham, The spectral theory of periodic differential operators, Scottish Academic Press, Edinburgh, 1973.

[21] P. Kuchment, Floquet theory for partial differential equations, Oper. Theory Adv. Appl., 60, Birkhäuser, Basel, 1993.

[22] W. Magnus, S. Winkler, Hill's equation, Interscience Publishers, J. Wiley, New York, 1966.

[23] J. Weidmann, Spectral theory of ordinary differential operators, Lecture Notes in Math., 1258, Springer-Verlag, Berlin, 1987.

[24] E. L. Ince, "A linear differential equation with periodic coefficients", Proc. London Math. Soc. (2), 23 (1924), 56-74.

[25] E. M. Harrell II, "On the effect of the boundary conditions on the eigenvalues of ordinary differential equations", Contributions to analysis and geometry (Baltimore, MD, 1980), Johns Hopkins Univ. Press, Baltimore, MD, 1981, 139-150. 
[26] J. Avron, B. Simon, "The asymptotics of the gap in the Mathieu equation", Ann. Physics, 134:1 (1981), 76-84.

[27] A. Liapounoff, "Sur une série dans la théorie des équations différentielles linéaires du second ordre à coefficients périodiques", Записки Императорской академии наук по Физико-математическому отделению, 13:2 (1903).

[28] P. Djakov, B. Mityagin, "Asymptotics of instability zones of Hill operators with a two term potential", C. R. Math. Acad. Sci. Paris, 339:5 (2004), 351-354.

[29] P. Djakov, B. Mityagin, "Asymptotics of instability zones of the Hill operator with a two term potential", J. Funct. Anal. (to appear); arXiv: math-ph/0509034.

[30] М. Г. Гасымов, "Спектральный анализ одного класса несамосопряженных дифференциальных операторов второго порядка", Функи. анализ и его прилож., 14:1 (1980), 14-19.

[31] В. А. Ткаченко, "Спектральный анализ несамосопряженного оператора Хилла", Докл. АН СССР, 322:2 (1992), 248-252.

[32] J.-J. Sansuc, V. Tkachenko, "Spectral parametrization of non-selfadjoint Hill's operators", J. Differential Equations, 125:2 (1996), 366-384.

[33] V. Tkachenko, "Discriminants and generic spectra of nonselfadjoint Hill's operators", Spectral operator theory and related topics, Adv. Soviet Math., 19, Amer. Math. Soc., Providence, RI, 1994, 41-71.

[34] В. А. Марченко, Операторы Штурма-Лиувилля и их приложения, Наукова думка, Киев, 1977.

[35] J.-J. Sansuc, V. Tkachenko, "Spectral properties of non-selfadjoint Hill's operators with smooth potentials", A. Boutet de Monvel, V. Marčenko (eds.), "Algebraic and geometric methods in mathematical physics" (Kaciveli, 1993), Math. Phys. Stud., 19, Kluwer, Dordrecht, 1996, 371-385.

[36] T. Kappeler, B. Mityagin, "Gap estimates of the spectrum of Hill's equation and action variables for KdV", Trans. Amer. Math. Soc., 351:2 (1999), 619-646.

[37] T. Kappeler, B. Mityagin, "Estimates for periodic and Dirichlet eigenvalues of the Schrödinger operator", SIAM J. Math. Anal., 33:1 (2001), 113-152.

[38] P. Djakov, B. Mityagin, "Smoothness of Schrödinger operator potential in the case of Gevrey type asymptotics of the gaps", J. Funct. Anal., 195:1 (2002), 89-128.

[39] P. Djakov, B. Mityagin, "Spectral triangles of Schrödinger operators with complex potentials", Selecta Math. (N.S.), 9:4 (2003), 495-528.

[40] Т. Като, Теория возмущений линейных операторов, Мир, М., 1972.

[41] М. Рид, Б. Саймон, Методъ современной математической физики. Т. 2: Гармонический анализ. Самосопряженность, Мир, М., 1978.

[42] Б. М. Левитан, И. С. Саргсян, Введение в спектральную теорию: Самосопряженные обыкновенные дифференциальные операторы, Наука, М., 1970.

[43] И. М. Гельфанд, "Разложение по собственным функциям уравнения с периодическими коэффициентами”, Докл. АН СССР, 73:6 (1950), 1117-1120.

[44] Ф. С. Рофе-Бекетов, "О спектре несамосопряженных дифференциальных операторов с периодическими коэффициентами”, Докл. АН СССР, 152:6 (1963), 13121315.

[45] F.S. Rofe-Beketov, A. M. Kholkin, Spectral analysis of differential operators. Interplay between spectral and oscillatory properties, World Scientific Monograph Series in Mathematics, 7, World Scientific, Hackensack, NJ, 2005.

[46] Б. С. Митягин, "Сходимость разложений по собственным функциям оператора Дирака", Докл. РАН, 393:4 (2003), 456-459.

[47] B. Mityagin, "Spectral expansions of one-dimensional periodic Dirac operators", Dyn. Partial Differ. Equ., 1:2 (2004), 125-191. 
[48] М. И. Нейман-заде, А. А. Шкаликов, "Операторы Шрёдингера с сингулярными потенциалами из пространств мультипликаторов", Матем. заметки, 66:5 (1999), 723-733.

[49] А.М. Савчук, А.А. Шкаликов, "Операторы Штурма-Лиувилля с распределениями-потенциалами", Тр. МMO, 64 (2003), 159-212.

[50] М. А. Красносельский, Г. М. Вайникко, П. П. Забрейко, Я. Б. Рутицкий, В. Я. Стеценко, Приближенное решение операторных уравнений, Наука, М., 1969.

[51] G. Nenciu, R. Purice, "Onedimensional periodic Dirac Hamiltonians: semiclassical and high-energy asymptotics for gaps", J. Math. Phys., 37:7 (1996), 3153-3167.

[52] Г. Полиа, Г. Сегё, Задачи и теоремъ из анализа. Часть 1: Ряды. Интегральное исчисление. Теория функиий, Наука, М., 1978.

[53] S. Mandelbrojt, Series de Fourier et classes quasi-analytiques de fonctions, GauthierVillars, Paris, 1935.

[54] И. М. Гельфанд, Г. Е. Шилов, Пространства основных и обобщенных функиий, Обобщенные функции, 2, Физматлит, М., 1958.

[55] J. Pöschel, Hill's potentials in weighted Sobolev spaces and their spactral gaps, Preprint, Institut für Analysis, Dynamik und Optimierung, Universität Stuttgart, Stuttgart, 2004; http://www.poschel.de/pbl/w-gaps.pdf.

[56] V. Tkachenko, "Characterization of Hill operators with analytic potentials", Integral Equations Operator Theory, 41:3 (2001), 360-380.

[57] Г. М. Кесельман, "О безусловной сходимости разложений по собственным функциям некоторых дифференциальных операторов”, Изв. вузов. Матем., 1964, № 2, 82-93.

[58] В.П. Михайлов, “О базисах Рисса в $\mathscr{L}_{2}(0,1)$ ”, Докл. АН СCCP, 144:5 (1962), 981-984.

[59] А. С. Макин, "Об одной задаче на собственные значения для нелинейного уравнения Штурма-Лиувилля с краевыми условиями, зависящими от спектрального параметра", Докл. РАН, 399:2 (2004), 161-164.

[60] М. А. Наймарк, Линейные дифференииальные операторы, Наука, М., 1969.

[61] E. L. Ince, "A proof of the impossibility of the coexistence of two Mathieu functions", Proc. Cambridge Philos. Soc., 21 (1922), 117-120.

[62] E. Hille, "On the zeros of Mathieu functions", Proc. London Math. Soc. (2), 23 (1924), $185-237$.

[63] Z. Markovic, "On the impossibility of simultaneous existence of two Mathieu functions", Proc. Cambridge Philos. Soc., 23 (1926), 203-205.

[64] Н. В. Мак-Лахлан, Теория и приложения функиий Матъе, ИЛ, М., 1953.

[65] S. Winkler, W. Magnus, The coexistence problem for Hill's equation, Research Report No. BR-26, New York University, Institute of Mathematical Sciences, Division of Electromagnetic Research, 1958.

[66] D. M. Levy, J. B. Keller, "Instability intervals of Hill's equation", Comm. Pure Appl. Math., 16 (1963), 469-476.

[67] P. Djakov, B. Mityagin, "The asymptotics of spectral gaps of a 1D Dirac operator with cosine potential", Lett. Math. Phys., 65:2 (2003), 95-108.

[68] P. Djakov, B. Mityagin, "Multiplicities of the eigenvalues of periodic Dirac operators", J. Differential Equations, 210:1 (2005), 178-216.

[69] A. Grigis, "Estimations asymptotiques des intervalles d'instabilité pour l'équation de Hill”, Ann. Sci. École Norm. Sup. (4), 20:4 (1987), 641-672.

[70] E. T. Whittaker, "On a class of differential equations whose solutions satisfy integral equations", Proc. Edinburgh Math. Soc., 33 (1914-1915), 14-23.

[71] P. Djakov, B. Mityagin, "Simple and double eigenvalues of the Hill operator with a two-term potential", J. Approx. Theory, 135:1 (2005), 70-104. 
[72] А. В. Турбинер, "Квантовая механика: задачи, промежуточные по отношению к точнорешаемым и точнонерешаемым", ЖЭТФ, 94:2 (1988), 33-44.

[73] A. V. Turbiner, "Quasi-exactly-solvable problems and sl(2) algebra", Comm. Math. Phys., 118:3 (1988), 467-474.

[74] H. Volkmer, "Instability intervals of the Ince and Hill equations", Analysis, 25 (2005), 189-204.

[75] Л.А. Пастур, В.А. Ткаченко, "К спектральной теории операторов Шрёдингера с периодическими комплекснозначными потенциалами", Функи. анализ и его прилож., 22:2 (1988), 85-86.

[76] M. Simbirskii, "Inverse problem for the Sturm-Liouville operator with almost-periodic potential having only positive Fourier exponents", Entire and subharmonic functions, Adv. Soviet Math., 11, Amer. Math. Soc., Providence, RI, 1992, 21-38.

[77] K. C. Shin, "On half-line spectra for a class of non-self-adjoint Hill operators", Math. Nachr., 261-262 (2003), 171-175.

[78] P. Djakov, B. Mityagin, "Trace formula and spectral Riemann surfaces for a class of tri-diagonal matrices", J. Approx. Theory, 139:1-2 (2006), 293-326.

[79] P. Djakov, B. Mityagin, "Spectral gaps of the periodic Schrödinger operator when its potential is an entire function", Adv. in Appl. Math., 31:3 (2003), 562-596.

[80] В. А. Марченко, И. В. Островский, "Аппроксимация периодических потенциалов конечнозонными потенциалами", Вестн. Харък. гос. ун-та, 1980, № 205, 4-40.

[81] Т.В. Мисюра, "Характеристика спектров периодических и антипериодических краевых задач, порождаемых операторами Дирака. I, II”, Теория функиий, функииональный анализ и их приложения, вып. 30, 31, Харьк. ун-т, Харьков, 1978, 1979, 90-101, 102-109.

[82] V. Tkachenko, "Non-selfadjoint periodic Dirac operators with finite-band spectra", Integral Equations Operator Theory, 36:3 (2000), 325-348.

[83] V. Tkachenko, "Non-selfadjoint periodic Dirac operators", Operator theory, system theory and related topics (Beer-Sheva/Rehovot, 1997), Oper. Theory Adv. Appl., 123, Birkhäuser, Basel, 2001, 485-512.

[84] B. Grébert, T. Kappeler, "Density of finite gap potentials for the Zakharov-Shabat system", Asymptotic Anal., 33:1 (2003), 1-8.

\section{П. Джаков (P. Djakov)}

Поступила в редакцию

Sofia University St. Kliment Ohridski, Bulgaria

23.04 .2006

E-mail: djakov@fmi.uni-sofia.bg

Б. С. Митягин (B.S. Mityagin)

The Ohio State University, USA

E-mail: mityagin.1@osu.edu 